\title{
Controlled radical polymerization at pressures up to 2000 bar
}

\author{
Dissertation \\ zur Erlangung des Doktorgrades \\ der Mathematisch-Naturwissenschaftlichen Fakultäten \\ der Georg August Universität zu Göttingen
}

vorgelegt von

Eric Minaux

aus Remiremont, Frankreich

Göttingen 2001 
D 7

Referent: $\quad$ Prof. Dr. M. Buback

Korreferent: Prof. Dr. K. Hoyermann

Tag der mündlichen Prüfung: den 3. Mai 2001 


\section{TABLE OF CONTENTS}

\section{Abstract}

1. INTRODUCTION

2.1 Classifications of CRPs 5

$\begin{array}{lr}2.2 \text { Persistent radical effect } & 8\end{array}$

2.3 Nitroxide-mediated polymerization 9

$\begin{array}{ll}2.4 \text { Polymerization rate } & 10\end{array}$

2.5 Extension for high pressure $\quad 12$

$\begin{array}{ll}2.6 \text { References } & 13\end{array}$

$\begin{array}{ll}\text { 3. EXPERIMENTAL } & 17\end{array}$

$\begin{array}{ll}3.1 \text { Apparatus } & 17\end{array}$

$\begin{array}{ll}3.1 .1 \text { Optical high-pressure cell } & 17\end{array}$

$\begin{array}{ll}3.1 .2 \text { Heating and temperature control } & 18\end{array}$

$\begin{array}{ll}\text { 3.1.3 FT-IR/NIR Spectrometer } & 19\end{array}$

$\begin{array}{ll}3.1 .4 \text { Pressure generation and control } & 19\end{array}$

$\begin{array}{ll}3.2 \text { Overall assembly } & 20\end{array}$

3.2.1 Continuous flow method 20

3.2.2 Styrene and meth(acrylate) polymerization 21

3.2.3 Ethene polymerization $\quad 22$

3.3 SEC-experiments 23

3.4 Simulation tool PREDICI ${ }^{\circledR}$

3.5 Chemicals 24

3.5.1 Nitroxides and alkoxyamines $\quad 24$

3.5.2 Peroxides 25

$\begin{array}{ll}\text { 3.5.3 Monomers } & 26\end{array}$ 
$\begin{array}{ll}3.6 \text { Performance of a typical experiment } & 27\end{array}$

3.6.1 Continuous flow method - peroxide decomposition 27

3.6.2 Styrene and (meth)acrylate polymerization $\quad 28$

$\begin{array}{ll}3.6 .3 \text { Ethene polymerization } & 29\end{array}$

3.7 Error estimate $\quad 30$

$\begin{array}{ll}3.8 \text { References } & 30\end{array}$

4. KINETIC MODELS AND THEORETICAL PREDICTIONS 33

4.1 Kinetic model for controlled styrene homopolymerization. General 33 description and development of a strategy for parameter estimations

4.1.1 Elementary reactions in nitroxide-mediated polymerization of styrene 33

4.1.2 Pressure dependence of TEMPO-mediated styrene polymerization 36

$\begin{array}{ll}\text { 4.1.3 Spontaneous styrene initiation } & 38\end{array}$

4.1.4 Simulation of controlled styrene polymerization at high pressure $\quad 40$

4.2 Kinetic model for nitroxide -mediated ethene homopolymerization 45

4.2.1 Elementary reactions in free-radical ethene homopolymerization 45

4.2.2 Rate constants in free-radical ethene homopolymerization 49

4.2.3 Introduction of reversible nitroxide radical trapping reactions $\quad 51$ into the model for ethene free-radical polymerization

4.2.4 Simulation of ethene polymerization in the presence of 52 a TEMPO-based alkoxyamine

4.2.5 Persistent radical effect as described by Fischer $\quad 54$

4.2.6 Aspects of control in ethene polymerization in the presence of nitroxide 57

4.3 References $\quad 62$

5 EXPERIMENTAL OBSERVATIONS

$\begin{array}{ll}\text { 5.1 IR/NIR-experiments } & 65\end{array}$

$\begin{array}{ll}\text { 5.2 Study of the initiation step } & 66\end{array}$

5.2.1 Peroxide decomposition in $n$-heptane 66

5.2.2 Peroxide decomposition in the presence of TEMPO in $n$-heptane $\quad 70$

$\begin{array}{ll}\text { 5.2.3. Quantitative IR Spectroscopy } & 71\end{array}$ 
5.2.4. Evaluation of first-order rate coefficients

5.2.5. Tabulated first-order rate coefficients $\quad 74$

$\begin{array}{ll}5.3 \text { Nitroxide-mediated styrene polymerization } & 76\end{array}$

5.3.1 Styrene CRP with SG1 77

$\begin{array}{ll}\text { 5.3.2 Styrene CRP with TEMPO } & 80\end{array}$

5.3.3 Styrene CRP with DPAIO $\quad 82$

5.4 (Meth)acrylate CRP under high pressure $\quad 84$

5.4.1 Butyl acrylate CRP with SG1 84

5.4.2 Nitroxide-mediated methyl methacrylate polymerization 86

5.5 Nitroxide-mediated ethene polymerization under high pressure $\quad 90$

5.5.1 Bulky nitroxides : DTBN, SG1 90

5.5.2 Commercially available nitroxides : TEMPO and TEMPO-derivatives 93

5.5.3 Thermal nitroxide decomposition 98

5.5.4 Newly developed nitroxides : DPAIO and derivatives 100

$\begin{array}{ll}\text { 5.6 RAFT ethene polymerization } & 103\end{array}$

$\begin{array}{ll}5.7 \text { References } & 106\end{array}$

$\begin{array}{ll}\text { 6. DiSCUSSION } & 109\end{array}$

6.1 Study of thermal peroxide decomposition 109

6.2. Nitroxide mediated styrene polymerization 113

6.2.1 Comparison of TEMPO and SG1 as mediators of styrene polymerization 113

$\begin{array}{ll}\text { 6.2.2 DPAIO-mediated styrene polymerization } & 115\end{array}$

$\begin{array}{ll}\text { 6.2.3 Effect of the initiating system } & 116\end{array}$

$\begin{array}{ll}\text { 6.2.4 Advantages of high pressure in styrene CRP } & 118\end{array}$

$\begin{array}{ll}\text { 6.2.5 Effect of low initiator concentration on styrene CRP } & 121\end{array}$

6.2.6 Simulation of TEMPO-mediated styrene polymerization 124 under high pressure

$\begin{array}{lr}\text { 6.2.7 Simulation of SG1-mediated styrene CRP } & 128\end{array}$

6.3 Nitroxide-mediated (meth)acrylate polymerization 132

$\begin{array}{ll}\text { 6.3.1 Nitroxide-mediated butyl acrylate polymerization } & 132\end{array}$

6.3.2 Nitroxide-mediated methyl methacrylate polymerization 133

6.4 Nitroxide-mediated ethene polymerization under high pressure 141

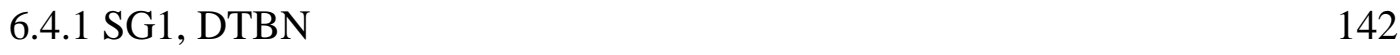


6.4.2 TEMPO and TEMPO-derivatives

6.4.3 Thermal decomposition of nitroxides

6.4.5 Determination of the cleavage temperature of alkoxyamines

6.4.6 Study of BDE by molecular modeling of a series of indolinic aminoxyl radicals

6.5 RAFT ethene polymerization under high pressure 


\begin{abstract}
The control of free-radical polymerization at high pressure has been intensively investigated. Special attention has been paid to high-pressure ethene polymerization. The high pressure studies were also carried out for styrene homopolymerization as an extended body of literature is available on kinetic aspects of the ambient pressure styrene polymerization.
\end{abstract}

Using quantitative Fourier transform infra-red (FTIR) spectroscopy, in a wide temperatures and pressures range, rate coefficients have been measured for the decomposition of peroxides in the presence of the persistent radical TEMPO. Neither the first-order rate coefficients, $k$, nor the activation energies for TAPP and TBPA differ in decomposition experiments with and without TEMPO. The activation energies determined are in excellent agreement with literature data, so that it is assumed that the presence of nitroxide does not interfere in perester decomposition over a wide range of experimental conditions.

The influence of high pressure on controlled polymerization was studied for styrene polymerizations in the presence of several mediators. High pressure induces a rate enhancement of styrene polymerization with SG1 and TEMPO. Narrow polydispersities and molecular weights close to theoretical predictions are observed irrespective of the pressure applied, especially at high initiator concentration. The combination of high pressure and of a bulky nitroxide, such as SG1, induces an approximately nine time faster polymerization rate at 2000 bar as compared to the classical TEMPO-mediated styrene polymerization at 1 bar.

High propagation rate and low termination rate at 2000 bar allow for styrene CRP with reasonable rates even at lower temperature. SG1 and TEMPO-mediated styrene polymerizations were carried out at 2000 bar, $100^{\circ} \mathrm{C}$ and $115^{\circ} \mathrm{C}$, respectively. Typically, polydispersities close to 1.3 are obtained above $30 \%$ styrene conversion. PREDICI ${ }^{\circledR}$ simulations satisfactorily represent the molecular weight distributions obtained and also predict a good control of TEMPO-mediated styrene polymerization at temperatures up to $95^{\circ} \mathrm{C}$.

Styrene CRPs were carried out at low initiator concentration and at high conversion where high molecular weight may be obtained. At 50 bar and below, the molar mass of 
polystyrene from CRP reaches an upper limiting value of about $70000 \mathrm{~g} \cdot \mathrm{mol}^{-1}$, whereas at higher pressure high molecular weight material of narrow polydispersity may be obtained.

PREDICI $^{\circledR}$ simulations were performed in order to estimate the dissociation and combination rate coefficients of the reversible dissociation of the alkoxyamine under high pressure. Rate coefficients were found to be in the same order of magnitude as the ones determined for the polymeric analogues.

SG1-mediated butyl acrylate polymerization were also successfully controlled under high pressure and high molecular weight material with narrow polydispersity were obtained. On the other hand, MMA polymerization in the presence of SG1 and DPAIO was not controlled even under high pressure. The disproportionation reaction of growing radical with SG1 competes with combination. PREDICI ${ }^{\circledR}$ simulations suggest that MMA polymerization with DPAIO being not controlled is due to the low dissociation rate coefficient of DPAIOMMA.

Controlling high-pressure ethene polymerization regulated by persistent radicals is much complicated than styrene or acrylate polymerization. First of all, drastic temperature and pressure conditions are required for ethene polymerization and growing ethyl radicals are very reactive. All attempts to control high-pressure ethene polymerization by nitroxides failed. The information about control of ethene high-pressure polymerization contained in a patent from 1995 by Georges at al. could not be confirmed.

The highly reactive nitroxides SG1 and DTBN are not able to control ethene polymerization due to their low thermal stability. Polymerizations were found to be inhibited as long as the persistent radical is in excess. Thereafter a low polymerization rate is found which results from initiation of the alkoxyamine. The molecular weights are determined by termination and transfer processes.

TEMPO and hexyl-TEMPO were found to be more stable than the bulky nitroxides. Nevertheless, molecular weights and polydispersities show that TEMPO-mediated ethene polymerization is not controlled. It was observed that TEMPO traps growing radicals. HexylTEMPO, as expected from the low $k_{\mathrm{d}}$ value for hexyl-TEMPO dissociation, was shown to be a very stable alkoxyamine and very high temperature is required for cleavage of the dormant species. Under theses conditions, TEMPO will undergo thermal decomposition, that avoids 
any control of ethene polymerization. Moreover, transfer reactions at these high temperatures exclude controlled polymerization.

ESR investigations showed a good thermal stability of the newly developed nitroxide DPAIO at $200^{\circ} \mathrm{C}$ for many hours, but ethene polymerization in the presence of DPAIO resulted in broad MWDs.

PREDICI $^{\circledR}$ simulations and ab-initio calculations allow to understand the observed phenomena. The rate coefficient for the cleavage of dormant species in ethene TEMPOmediated controlled polymerization was shown to be much lower than with styryl-TEMPO. The high activation energy of $k_{\mathrm{d}}$, around $180 \mathrm{~kJ} \cdot \mathrm{mol}^{-1}$, demonstrates the strength of the alkylTEMPO bond which strongly shifts the equilibrium to the side of the dormant species. Thus the large excess of TEMPO which is required for effective control of the polymerization can not be obtained.

$\mathrm{Ab}$-initio calculations allowed for the estimation of the cleavage temperature of alkoxyamines. A cleavage temperature of $230^{\circ} \mathrm{C}$ is expected for hexyl-DPAIO. It is assumed that the alkoxyamine hexyl-DPAIO undergoes a thermal degradation of the $\mathrm{N}-\mathrm{O}$ bond at temperatures much lower than the ones required to cleave the $\mathrm{CO}$ bond. The bond dissociation energies of the $\mathrm{C}-\mathrm{O}$ and $\mathrm{N}-\mathrm{O}$ bonds were investigated for different alkoxyamines. The BDEs of indolinic aminoxyl compounds show that the $\mathrm{N}-\mathrm{O}$ bond is indeed weaker than the $\mathrm{CO}$ bond. Thus the NO bond of hexyl-DPAIO is assumed to predominantly cleave resulting in uncontrolled ethene polymerization.

RAFT ethene polymerization were also investigated. The idea behind these studies is that $\beta$-scission of the intermediate radical might work more selectively than does bond scission of alkoxyamines in which an ethylene type species is linked to a nitroxide. Molecular weights and polydispersities obtained are not typical for controlled polymerization. The MWDs observed are much narrower than the ones in nitroxide-mediated ethene polymerization. The RAFT agent used so far seems to primarily act as a chain-transfer agent. Nevertheless, only via the RAFT procedure a chance is still seen to control ethene polymerization. 


\section{INTRODUCTION}

An enormous interest in controlled free-radical polymerization, CRP, has emerged during recent years. Living polymerization was first defined by Szwarc ${ }^{1}$ as a chain growth process without chain breaking reactions (transfer and termination). Such a polymerization provides end-group control and enables the synthesis of block copolymers by sequential monomer addition. However, it does not necessarily provide molecular weight control and narrow molecular weight distributions (MWDs). Additional requirements to achieve such properties are that the initiator should be consumed at early stages of the polymerization and that exchange between species of various reactivities is fast in comparison with propagation ${ }^{2-}$ 6. If these additional criteria are observed, a controlled polymerization results. Controlled polymerization may also include side reactions, but only to an extent which does not considerably disturb the control of molecular structure of the polymer chain. Ideally controlled/living systems lead to polymer whose the degree of polymerization is predetermined by the ratio of the concentrations of consumed monomer to the initial initiator $D P_{\mathrm{N}}=\Delta[\mathrm{M}] /[\mathrm{I}]_{0}$, whose polydispersity is close to a Poisson distribution $\left(D P_{\mathrm{W}} / D P_{\mathrm{N}} \approx 1+1 / D P_{\mathrm{N}}\right)$, and whose all chains are end-functionalized. The best way to monitor such systems is by following the kinetics of polymerization and the evolution of molecular weights, polydispersities and functionalities with conversion. Three types of controlled/living radical polymerization have emerged which differ by the mechanism of exchange between active and dormant species : reversible homolytic cleavage of covalent species (nitroxides, iniferters, ATRP...), reversible formation of persistent hypervalent radicals (persistent radical effect) and degenerative transfer.

A wide variety of studies concerning controlled radical polymerization has been carried out with must experiments performed on styrene. With the exception of a patent ${ }^{7}$, strictly no study has been devoted to controlled radical polymerization under high pressure so far. Inspection of the formula (Eq. 1.1), which relates the reaction time for $90 \%$ monomer conversion, $t_{90}$, to the kinetic parameters of a CRP shows that the application of high pressure should be favorable. In this equation, which has been deduced by Fischer ${ }^{8}, k_{\mathrm{p}}$ and $k_{t}$ are respectively the propagation and termination rate coefficients, $K$ is the equilibrium constant ( $K$ $=k_{\mathrm{d}} / k_{\mathrm{c}}$ ) and $[\mathrm{I}]_{0}$ is the initial concentration of initiator. According to equation $1.1, t_{90}$ should 
be reduced at high pressure as the propagation rate coefficient is increased and the termination rate coefficient is decreased toward higher pressure.

$$
t_{90} / s=\frac{(2 \ln 10)^{3 / 2}}{3 k_{\mathrm{p}}^{3 / 2}}\left(\frac{k_{\mathrm{t}}}{K[I]_{0}}\right)^{1 / 2}
$$

The only one report on high-pressure controlled polymerization is a U.S. patent ${ }^{7}$ which describes a free-radical polymerization process to prepare a thermoplastic resin, by heating a mixture of a free-radical initiator, a stable free-radical agent and ethene at temperatures between 40 and $500^{\circ} \mathrm{C}$ and pressures from 500 to 5000 bar.

The aim of the present work is to study in detail the influence of high pressure on nitroxide-mediated controlled free radical polymerization. Special attention will be paid to high-pressure ethene polymerization. Several nitroxides have been selected, which largely differ in reactivity and in stability of the alkoxyamines. The high-pressure studies are also carried out for styrene homopolymerization as an extended body of literature is available on kinetic aspects of the ambient pressure styrene polymerization. Extended kinetic schemes for the high-pressure CRP of ethene and styrene will be presented. The experimental results will be accompanied by modeling via the program package PREDICI ${ }^{\circledR}$. Simulations are carried out for temperatures from 150 to $230^{\circ} \mathrm{C}$ for ethene and from 95 to $125^{\circ} \mathrm{C}$ for styrene at pressures up to 2000 bar.

Section 2 gives a brief introduction into aspects of controlled free-radical polymerization. The basic types of CRPs are described, depending on the chemistry of exchange and structure of the dormant species ${ }^{9}$ and the requirements for controlled/living radical polymerization are summarized. The activation processes and polymerization rates, $r_{\mathrm{p}}$, are also discussed.

Section 3 illustrates the experimental setups that have been used in the on-line vibrational spectroscopic analysis of polymerization under high pressure and briefly reports on the commercial program PREDICI ${ }^{\circledR}$ that has been used for the simulations. 
In section 4, kinetic models of nitroxide-mediated styrene and ethene homopolymerization are detailed and computer simulations, essential for the development of the experimental strategy, are performed.

In Section 5, the experimental data for styrene homopolymerization and for a few (meth)acrylate homopolymerizations are summarized. The major part of this chapter refers to the experimental investigation of ethene high-pressure polymerization in the presence of nitroxides and of a RAFT agent.

In Section 6, the experimental data are discussed and polymerization kinetics and product properties are estimated by PREDICI ${ }^{\circledR}$ simulations.

\subsection{References}

[1] M. Szwarc, Nature 1956, 176, 1168

[2] R. Quirk, B. Lee, Polym. Int. 1992, 27, 359

[3] K. Matyjaszewski, J. Phys. Org. Chem. 1995, 8, 197

[4] K. Matyjaszewski, Ed. Cationic Polym. : Mechanisms, Synthesis and Applications; Marcel Dekker : New York, 1996

[5] K. Matyjaszewski, C. Lin, Makromol. Chem. Macromol. Symp. 1991, 47, 221

[6] A. Müller, R. Zhuang, D. Yan, G. Litvinienko, Macromolecules 1995, 28, 4326

[7] K. Moffat, M. Saban, R. Veregin, M. Georges, G. Hamer, P. Kazmaier, U.S. Patent 5 449724,1995

[8] H. Fischer, J. Polym. Sc. Part A : Polym. Chem. 1999, 37, 1886

[9] K. Matyjaszewski, ACS Symp. : Controlled/Living Radical Polymerization 2000, 768, 2 


\section{ASPECTS OF CONTROLLED FREE-RADICAL POLYMERIZATION}

Controlled/living radical polymerization follows the principle of equilibration between growing free radicals and dormant species. There are several approaches to control FRP by suppressing the contribution of chain breaking reactions and assuring quantitative initiation ${ }^{1}$, two conditions necessary to achieve good control over molecular weights, polydispersities and end functionalities. The reactions are described as controlled free-radical polymerizations (CRP) or controlled/living radical polymerizations rather than as true living polymerization, due to the presence of unavoidable termination reactions. Currently three methods appear to be very efficient and can be successfully applied to a large number of monomers : stable freeradical polymerization (SFRP), best represented by nitroxide-mediated polymerization (NMP), metal catalyzed atom transfer radical polymerization (ATRP), and reversible addition-fragmentation chain transfer (RAFT) along with other degenerative transfer processes.

\subsection{Classification of CRPs}

Basically, each type of controlled radical polymerization is based on the principle of dynamic equilibration between dormant and active species. This equilibrium defines the observed rates and physical properties of the polymer. It is preferable to distinguish CRPs according to mechanistic aspects, as thus enables better correlation of rates, molecular weights and polydispersities with the concentration of the involved species.

In all cases, dormant (non-propagating) species are reversibly activated with the rate constant of activation (or rate constant of dissociation $k_{\mathrm{d}}$ ) to form the active species (radical $\mathrm{R}_{\mathrm{n}}$ ), which reacts with monomer $\mathrm{M}$ with the propagation rate constant $k_{\mathrm{p}}$. The propagating radicals are deactivated with the rate constant of deactivation (or rate constant of combination $k_{\mathrm{c}}$ ), or can terminate with other growing radicals with the rate constant of termination $k_{\mathrm{t}}$. In all CRPs, the concentration of growing radicals is kept very low, in order to reduce significantly termination. Well-controlled systems should provide : 
- a linear semilogarithmic plot of $\ln \left([M]_{0} /[M]\right)$ vs time, in cases where the reaction is first order in monomer concentration. Acceleration with respect to a linear relation indicates slow initiation whereas deceleration indicates termination or deactivation of the catalyst

- linear evolution of molecular weight distribution with conversion. Lower MWD than predicted by the ratio $\mathrm{DP}_{\mathrm{N}}=\Delta[\mathrm{M}] /[\mathrm{I}]_{0}$ indicates transfer and higher MWD indicates inefficient initiation or chain coupling

- polydispersities that decrease with conversion. Significant chain termination induces an increase of polydispersity with conversion

- end functionalities that are not affected by slow initiation and exchange but are reduced when chain breaking reactions become important

Time and conversion dependencies for ideal controlled/living systems and the corresponding deviations are schematically illustrated in Fig. 2.1.
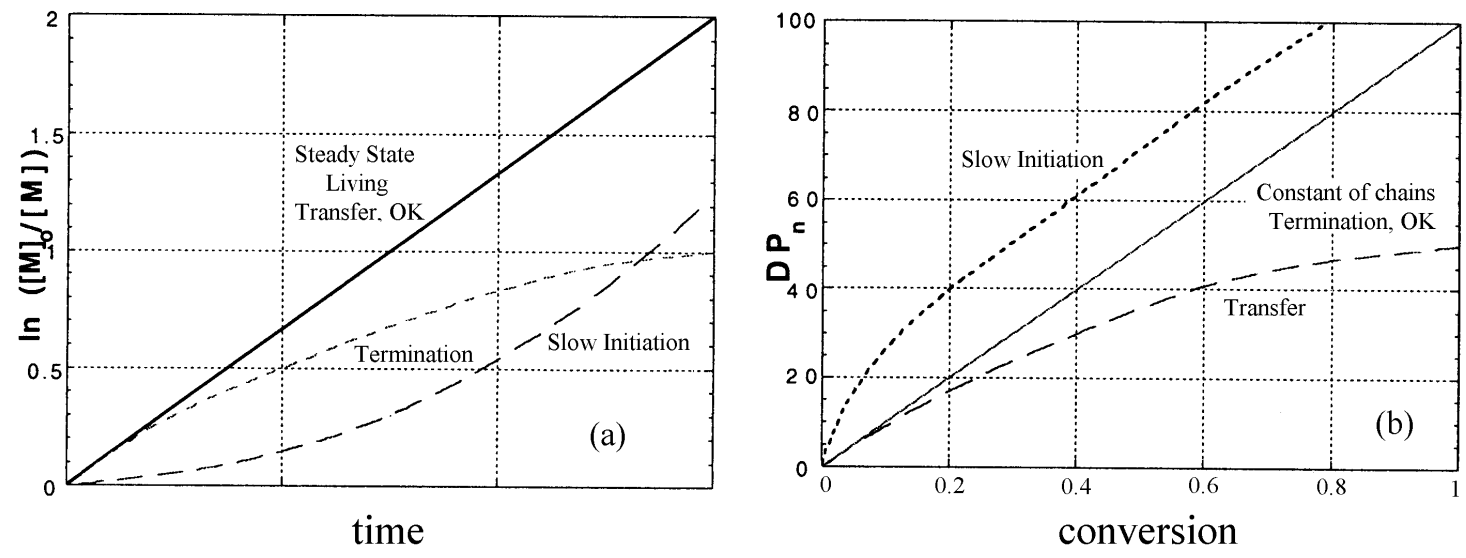

Fig. 2.1. Schematic effect of slow intiation, transfer, termination and exchange on kinetics (a) and molecular weights (b) for controlled/living systems.

Controlled-radical polymerization may be classified in four different cases. Case 1 is best illustrated by nitroxide-mediated polymerization in the presence of commercially available nitroxides such as TEMPO ${ }^{2,3}$ and TEMPO-derivatives ${ }^{4}$ or DTBN ${ }^{5}$, and new bulky acyclic nitroxides ${ }^{6,7}$, triazolinyl radicals ${ }^{8}$, some bulky organic radicals ${ }^{9,10}$, compounds with photolabile $\mathrm{C}-\mathrm{S}$ bonds ${ }^{11}$ and organometallic species ${ }^{12,13}$. Case 2 is based on the catalyzed, reversible cleavage of the covalent bond in the dormant species via a redox process. The key step in controlling the polymerization is the atom transfer between growing radicals and a catalyst. This process is named atom transfer radical polymerization (ATRP) and is catalyzed 
by various $\mathrm{Ru}, \mathrm{Cu}, \mathrm{Fe}$ and other transition metal derivatives ${ }^{14-18}$. The process described in case 3 involves the reversible formation of persistent radicals (PR) by reaction of growing radicals with a species containing an even number of electrons. PRs do not terminate or propagate with monomer. The role of reversible radical trap may be played by phosphites ${ }^{19}$ or reactive but non polymerizable alkenes ${ }^{20,21}$. Case 4 is based on a thermodynamically neutral exchange process between a growing radical, present at very low concentration, and dormant species, present at much higher concentration (at least by three or four orders of magnitude). This degenerative transfer can employ alkyl iodides ${ }^{22}$, unsaturated methacrylate esters ${ }^{23}$ or thioesters ${ }^{24}$.
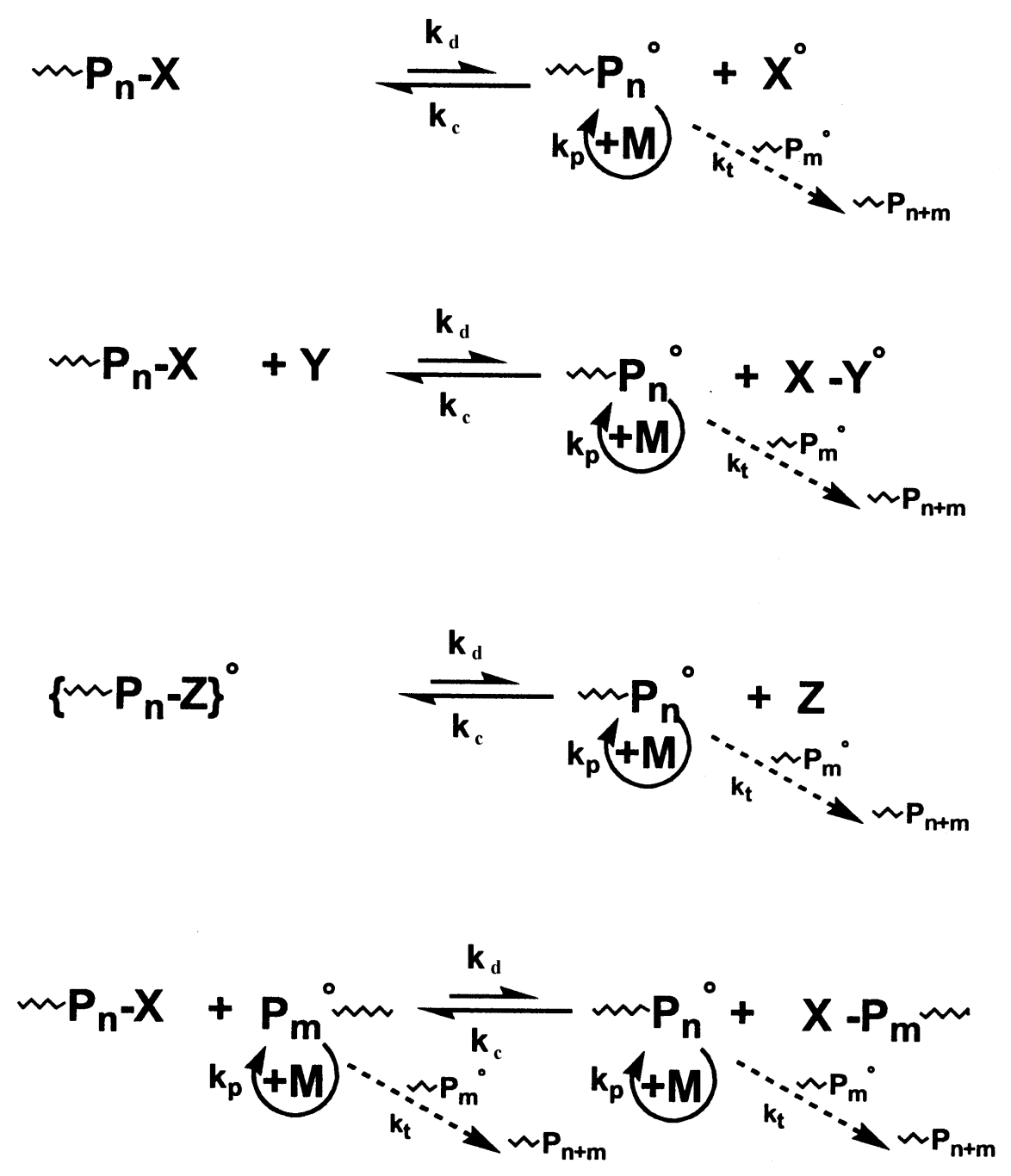

Fig. 2.2. Schematic description of different mechanisms involving controlled radical polymerization 
In cases 1 to 3 the equilibrium is strongly shifted towards the dormant (nonpropagating) species and polymerization rate depends on the concentration of the CRP mediating species. Case 4 is very different : there is no persistent radical (PR). The equilibrium constant $K$ is unity $\left(k_{\mathrm{c}}=k_{\mathrm{d}}=k_{\mathrm{exch}}\right)$ and rates should conform to conventional radical systems with a reaction order of 1; the initiator. Each system has a specific dependence of polymerization rate, molecular weight and polydispersity on conversion and on concentrations of the involved reagents ${ }^{25}$.

\subsection{Persistent radical effect}

The living/controlled character was shown to be closely related to a general phenomenon which appears in reactions where transient and persistent radicals are formed simultaneously, and which is referred to as the persistent radical effect ${ }^{26-28}$ (PRE). Most CRP systems (cases 1 to 3 ) conform to the PRE model. Systems based on degenerative transfer, including RAFT polymerization do not conform to the PRE model. In cases where the persistent radical effect applies, the control and the rate of polymerization depend on the size of the equilibrium constant $K\left(K=k_{\mathrm{d}} / k_{\mathrm{c}}\right)$ and particularly on the value of the dissociation constant $k_{\mathrm{d}}$. Hence, in nitroxide-mediated polymerization, the bond dissociation energy $(\mathrm{BDE})$ of the $\mathrm{NO}-\mathrm{C}$ bond is a key parameter.

The major aspects of the persistent radical effect have been thoroughly investigated by Fischer ${ }^{27}$. Control by reversible bond cleavage will now be considered as the mode of control that has mostly been used within the present work. The reaction may be started by an initiator species $\mathrm{R}_{0}-\mathrm{N}$ which decomposes into a transient and into a persistent radical. The radical concentration is zero at time zero. The transient radical grows by propagation. Initially, the concentrations of both radical species increase linearly, at a rate which is given by the decomposition rate coefficient, $k_{\mathrm{d}}$. The radical concentrations increase until the rate of the bimolecular back reaction, $k_{\mathrm{c}}$, and the self termination of the transient radicals, $k_{\mathrm{t}}$, compete with radical formation. Because of the asymmetry of the radical coupling pathways, the persistent radicals accumulate and, in contrast to conventional radical polymerization, the radical concentrations do not reach simultaneously quasi-steady states. This happens only at infinite time when the concentration of transient radicals has dropped to zero. Then, the 
concentration of the persistent species reaches the final level which equals the initial initiator concentration. In addition, all transient radicals have terminated to unreactive products, and there are no dormant chains left.

From the initial and from the long-time behavior one may conclude that the concentration of transient species reaches an intermediate maximum. Before this maximum is reached the initiating molecules $\left(\mathrm{R}_{0}-\mathrm{N}\right)$ acquire several monomer units $\left(\mathrm{R}-(\mathrm{M})_{\mathrm{n}}-\mathrm{N}\right)$. Thereafter, the concentration of transient radicals decreases whereas the persistent species accumulate further. Under certain conditions defined by Fischer ${ }^{28}$, the radical concentrations, $[R]$ and $[N]$ respectively growing and persistent radicals, obeys the rather unusual rate laws

$$
[\mathrm{R}]=\left(\frac{K[\mathrm{I}]_{0}}{3 k_{\mathrm{t}}}\right)^{1 / 3} t^{-1 / 3} \quad[\mathrm{~N}]=\left(3 k_{\mathrm{t}} K^{2}[\mathrm{I}]_{0}\right)^{1 / 3} t^{1 / 3}
$$

which holds in an extended time region. Both $[\mathrm{R}]$ and $[\mathrm{N}]$ are time dependent, due to the termination reaction.

\subsection{Nitroxide-mediated polymerization}

The N-oxyl-controlled free-radical polymerization is one of the most extensively studied and used methods in controlled free-radical polymerization. The key reaction of this type of polymerization is the reversible deactivation of the growing radical, $\mathrm{R}$, by an $\mathrm{N}$-oxyl compound, N, :

$$
\mathrm{R}+\mathrm{N} \underset{k_{\mathrm{d}}}{\stackrel{k_{\mathrm{c}}}{\rightleftarrows}} \mathrm{R} \mathrm{N} \quad K=k_{\mathrm{d}} / k_{\mathrm{c}}
$$

where $k_{\mathrm{c}}$ and $k_{\mathrm{d}}$ are the rate constants of combination and dissociation, respectively. The simultaneous initiation, together with the small contributions of transfer and of irreversible termination result in controlled molecular weights and narrow molecular weight distributions.

$\mathrm{N}$-oxyl species can be employed according to three different methods. The first method uses a combination of a nitroxide and a conventional initiator, e.g. AIBN, or a peroxide, e.g. BPO or dicumylperoxide ${ }^{29-37}$. Radicals formed by decomposition of the 
initiator starts the polymeric growth and the $\mathrm{N}$-oxyls deactivate reversibly these growing radicals. The second method consists of using an alkoxyamine or a polyalkoxyamine ${ }^{38-52}$ such as [polymer-N-oxyl] adducts synthesized by N-oxyl-radical controlled polymerization. In this method no excess of nitroxide is used which can slow down the polymerization rate. The third method allows a $\mathrm{N}-$ oxyl controlled polymerization process ${ }^{51-57}$ in the absence of any additional initiating system. This procedure may be used with monomers undergoing a thermal spontaneous initiation, e.g. styrene.

In case of polymerizations carried out in the presence of an initiator/nitroxide or alkoxyamine system, two types of radicals are formed which strongly differ in reactivity. The more reactive radical, $\mathrm{R}$ issued from the initiation step, starts the polymerization by reacting with monomer, and the less reactive one, $\mathrm{N}$, can only react by combination with a reactive radical (such as the growing radical). This last reaction is reversible and allows, at sufficiently high temperature, the reformation of free radicals which may further propagate. According to Fukuda ${ }^{44}$, the polymerization of the adduct $\mathrm{P}-\mathrm{N}$ is thermally initiated and the polymerization rate depends on the equilibrium given in Eq. 2.2. Consequently, some thermal polymerization of the monomer and a low dissociation enthalpy for the bonding $\mathrm{N}$-oxyl - polymer chain are recommendable.

The mechanism of the $\mathrm{N}-$ oxyl controlled radical polymerization is already well known 42,44,58 and computational models can satisfactorily describe experiments started with $\mathrm{N}$-oxyl - adducts ${ }^{41,44,59}$. The modeling of the controlled polymerization started with an initiator and nitroxide was extended by Schmidt-Naake et al. ${ }^{60}$.

\subsection{Polymerization rate}

The rate of nitroxide-mediated styrene polymerization $\left(r_{\mathrm{p}}\right)$ is well understood. Catala et al. ${ }^{40}$ reported experimental data which showed that $r_{\mathrm{p}}$ is independent of the concentration of the adduct S-DTBN (see section 3.5.1) used as the initiator. This was interpreted by Fukuda et al. ${ }^{61}$ in terms of the stationary-state kinetics. From the individual reactions relative to styrene polymerization presented on Figure 4.1, only reactions 1, 2, 5, 6 and 7 are relevant to change in radical concentration. $[\mathrm{R}]$ and $[\mathrm{N}]$ should follow the differential equations 


$$
\begin{aligned}
& d[\mathrm{~N}] / d t=k_{\mathrm{d}}[\mathrm{RN}]-k_{\mathrm{c}}[\mathrm{R}][\mathrm{N}] \\
& d[\mathrm{R}] / d t=k_{\mathrm{d}}[\mathrm{RN}]-k_{\mathrm{c}}[\mathrm{R}][\mathrm{N}]+r_{\mathrm{i}}-k_{\mathrm{t}}[\mathrm{R}]^{2}
\end{aligned}
$$

where $r_{\mathrm{i}}$ is the thermal initiation rate. If $r_{\mathrm{i}}$ differs from zero, the system will reach a stationary state in which $\mathrm{d}[\mathrm{R}] / \mathrm{d} t=\mathrm{d}[\mathrm{N}] / \mathrm{d} t=0$ and

$$
\begin{aligned}
& {[\mathrm{R}]=\left(r_{\mathrm{i}} / k_{\mathrm{t}}\right)^{0.5}} \\
& {[\mathrm{~N}]=\left(k_{\mathrm{d}} / k_{\mathrm{c}}\right) \cdot[\mathrm{PN}] /[\mathrm{R}]}
\end{aligned}
$$

The stationary concentration of $\mathrm{R}$ is determined by the balance of the initiation and termination rates (equation 2.5). Otherwise, $[\mathrm{N}]$ depends not only on the stationary value of [R] but also of [RN] and on the $k_{\mathrm{d}} / k_{\mathrm{c}}$ ratio (equation 2.6). And the polymerization rate, $r_{\mathrm{p}}$, may be expressed as

$$
r_{\mathrm{p}}=k_{\mathrm{p}}[\mathrm{R}][\mathrm{M}]=\left(k_{\mathrm{p}} / k_{\mathrm{t}}^{1 / 2}\right) \cdot r_{\mathrm{i}}^{1 / 2} \cdot[\mathrm{M}]
$$

which is independent of $[\mathrm{RN}]$, as experimentally observed. The polymerization rate of a nitroxide-mediated system is determined by the balance of the initiation and termination rates as in a conventional system where thermal initiation and a radical initiator play essentially the same role. The reversible dissociation of $\mathrm{RN}$ is capable of controlling the chain-length distribution but not the stationary polymerization rate. As far as the number of chains that are thermally produced stays small compared to the number of dormant species molecules, initiation and termination reactions have a minor effect on the chain-length distribution. On the other hand, controlled polymerization does not successfully proceed even if the reversible dissociation of RN occurs, if there is no permanent thermal initiation. In this case, controlled polymerization has to be induced by a radical initiator ${ }^{44,29-50,61-63}$. The same conclusions regarding the role on the initiation process have been reached independently by Greszta and Matyjaszewski ${ }^{64}$.

This stationary-state model is expected to hold for degenerative type systems and was in fact observed for styrene polymerizations with several adducts PS-degenerative transfer agent ${ }^{65,66}$. 
The other case that has been discussed is the one with $r_{\mathrm{i}}=0$ which leads to Eq. 2.8

$$
[\mathrm{N}]^{3}-[\mathrm{N}]_{0}^{3}=3 \cdot k_{\mathrm{t}} \cdot\left(K[\mathrm{I}]_{0}\right)^{2} \cdot t
$$

The case where $[\mathrm{N}]$ is zero has been discussed first by Fischer ${ }^{27}$ and subsequently by Fukuda et al. ${ }^{67}$. It leads to the characteristic power-law behavior of conversion index $\ln \left([M]_{0} /[M]\right)$ :

$$
\ln \left([M]_{0} /[M]\right)=\left(\frac{3}{2}\right) \cdot k_{\mathrm{p}} \cdot\left(\frac{K[\mathrm{I}]_{0}}{3 k_{\mathrm{t}}}\right)^{1 / 3} \cdot t^{2 / 3}
$$

The time for $90 \%$ conversion of the monomer is

$$
t_{90}=\frac{(2 \ln 10)^{3 / 2}}{3 k_{\mathrm{p}}^{3 / 2}} \cdot\left(\frac{k_{\mathrm{t}}}{K[\mathrm{I}]_{0}}\right)^{1 / 2}
$$

This behavior has been observed in nitroxide-controlled polymerizations of styrene in which self initiation is unimportant $28,67,68$.

Both equations, Eq. 2.9 and 2.10, contain the termination constant. Hence, termination influences the polymerization rate, as it does in conventional polymerizations. However, in contrast to polymerizations with constant and slow initiation, Eq. 2.9 shows that the $\ln \left([M]_{0} /[M]\right)$ versus time relative in CRP is not linear but exhibits a downward curvature, due to decreasing concentration of propagating radicals.

\subsection{Extension for high pressure}

Computational models for controlled/living free-radical polymerization have not yet been extended to high pressure. Schmidt-Naake et al. ${ }^{60}$ presented a model for the $\mathrm{N}$-oxyl controlled radical polymerization of styrene and compared simulated with experimental data 
obtained at ambient pressure. This model was modified for controlled radical polymerizations initiated by nitroxide plus initiator or by alkoxyamines. This model is easily extended to CRP at high pressure by including known ${ }^{62}$ activation volumes of the propagation and termination step and estimated activation volumes for the formation and dissociation reaction of the dormant species.

The modeling of controlled high-pressure ethene polymerization is based on the detailed studies of Busch ${ }^{69}$ into individual reaction steps in conventional ethene polymerization which, in addition to propagation and termination, include transfer to monomer and to polymer as well as $\beta$-scission reactions.

\subsection{References}

[1] K. Matyjaszewski, ACS Symp. : Controlled Radical Polymerization 1998, 685, 2

[2] D. Salomon, E. Rizzardo, P. Ccioli, U.S. Patent 1986, 581, 429

[3] R. Veregin, M. Georges, G. Hamer, P. Kazmaier, G. Hamer, Macromolecules 1993, 26, 2987

[4] C. Han, S. Butz, G. Schmidt-Naake, Die Angew. Makromol. Chem. 1999, 265, 69

[5] T. Fukuda, T. Terauchi, Chemistry Letters 1996, 293

[6] D. Benoit, S. Grimaldi, J.P. Finet, P. Tordo, M. Fontanille, Y. Gnanou, ACS Symp. : Controlled Radical Polymerization 1998, 685, 225

[7] D. Benoit, V. Chaplinski, R. Braslau, C. Hawker, J. Am. Chem. Soc. 1999, 121, 3904

[8] M. Steenbock, M. Klapper, K. Muellen, C. Bauer, M. Hubrich, Macromolecules 1998, 31,5223

[9] E. Borsig, M. Lazar, M. Capla, S. Florian, Die Angew. Makromol. Chem. 1969, 9, 89

[11] T. Otsu, M. Yoshida, Macromol. Chem. Rapid. Commun. 1982, 3, 127

[12] B. Wayland, G. Pozmik, S. Mukerjee, M. Fryd, J. Am. Chem. Soc. 1994, 116, 7943

[13] H. Harwood. L. Arvanitopoulos, M. Greuel, ACS Polymer Preprints 1994, 35, 549

[14] M. Kato, M. Kamigaito, M. Sawamato, T. Higashimura, Macromolecules 1995, 28, 1721

[15] J. Wang, K. Matyjaszewski, J. Am. Chem. Soc. 1995, 117, 5614

[16] K. Matyjaszewski, Chem. Eur. J. 1999, 5, 3095 
[17] T.E. Patten, K. Matyjaszewski, Acc. Chem. Res. 1999, 5, 3095

[18] T.E. Patten, K. Matyjaszewski, Adv. Mater. 1998, 10, 901

[19] D. Greszta, D. Mardare, K. Matyjaszewski, ACS Polymer Preprints 1994, 35, 466

[20] M. Steenbock, M. Klapper, K. Muellen, N. Pinhal, M. Hubrich, Acta Polym., 1996, 47, 276

[21] H. Harwood, L. Christov, M. Guo, T. Holland, A. Huckstep, Macromol. Symp. 1996, 111,25

[22] K. Matyjaszewski, S. Gaynor, J. Wang, Macromolecules 1995, 28, 2093

[23] C.L. Moad, G. Moad, E. Rizzardo, S. Thang, Macromolecules 1996, 29, 7717

[24] J. Chiefari, Y. Chong, F. Ercole, J. Krstina, J. Jeffrey, T. Le, C.L. Moad, G. Moad, E. Rizzardo, S. Thang, Macromolecules 1998, 31, 5559

[25] K. Matyjaszewski, ACS Symp. : Controlled/Living Radical Polymerization 2000, 768, 2

[26] H. Fischer, J. Am. Chem. Soc. 1986, 108, 3925

[27] H. Fischer, Macromolecules 1997, 30, 5666

[28] H. Fischer, J. Polym. Sci. Part A: Polym. Chem. 1999, 37, 1885

[29] M. Georges, R. Veregin, P. Kazmaier, G.Hamer, Macromolecules 1993, 26, 2987

[30] M. Georges, R. Veregin, P. Kazmaier, G.Hamer, Macromolecules 1993, 26, 5316

[31] K. Matyjaszewski, S. Gaynor, D. Greszta, D. Mardare, T. Shigemoto, Macromol. Symp. 1995, 98, 73

[32] R. Puts, D. Sogah, Macromolecules 1996, 29, 3323

[33] E. Yoschida, T. Fujii, J. Polym. Sci. 1997, 35, 2371

[34] E. Yoschida, J. Polym. Sci. 1996, 34, 2937

[35] M. Georges, R. Veregin, P. Kazmaier, G.Hamer, Trends Polym. Sci. 1994, 2, 66

[36] M. Georges, R. Veregin, P. Kazmaier, G.Hamer, Macromolecules 1995, 28, 4391

[37] H. Baethge, S. Butz, G. Schmidt-Naake, Macromol. Rapid. Commun. 1997, 18, 911

[38] C. Hawker, J. Am. Chem. Soc. 1994, 116, 11185

[39] C. Hawker, J. Hedrick, Macromolecules 1995, 28, 2993

[40] J. Catala, F. Bubel, S. Hammouch, Macromol. Rapid. Commun 1995, 28, 8441

[41] J. Catala, S. Hammouch, Macromol. Rapid. Commun. 1996, 17, 683

[42] K. Matyjaszewski, D. Greszta, Macromolecules 1996, 29, 7661

[43] C. Hawker, G. Barclay, A. Orellana, J. Dao, W. Davenport, Macromolecules 1996, 29, 5245 
[44] T. Fukuda, T. Terauchi, A. Goto, K. Ohno, Y. Tsujii, T. Miyamoto, S. Kobatake, B. Yamada, Macromolecules 1996, 29, 6393

[45] H. Baethge, S. Butz, G. Schmidt-Naake, Macromol. Rapid. Commun. 1997, 18, 1049

[46] K. Ohno, M. Ejaz, T. Fukuda, T. Miyamoto, S. Shimizu, Macromol. Chem. Phys. 1998, 199, 291

[47] T. Fukuda, T. Terauchi, A. Goto, Y. Tsujii, T. Miyamoto, Y. Shimizu, Macromolecules 1996, 29, 3050

[48] N. Listigovers, M. Georges, P. Odell, B. Keoshkerian, Macromolecules 1996, 29, 8992

[49] A. Goto, T. Fukuda, Macromolecules 1997, 30, 4272

[50] A. Goto, T. Fukuda, Macromolecules 1997, 30, 5183

[51] E. Rizzardo, Chem. Aust. 1987, 54, 32

[52] C. Han, S. Butz, G. Schmidt-Naake, Angew. Makromol. Chem. 1999, 265, 69

[53] D. Mardare, K. Matyjaszewski, Polym. Prepr. (Am. Chem. Soc. Div. Polym. Chem.) 1994, 35, 778

[54] M. Georges, R. Veregin, P. Kazmaier, G.Hamer, Polym. Prepr. (Am. Chem. Soc. Div. Polym. Chem.), 1994, 35, 870

[55] W. Davenport, L. Michalak, E. Malmström, M. Mate, B. Kurdi, C. Hawker, G. Barclay, R. Sinta, Macromolecules 1997, 30, 1929

[56] T. Connolly, J. Scaiano, Tetrahedron Lett. 1997, 38, 1133

[57] C. Han, M. Drache, G. Schmidt-Naake, Angew. Makromol. Chem. 1999, 264, 73

[58] K. Matyjaszewski, Macromol. Symp. 1996, 111, 47

[59] D. Greszta, K. Matyjaszewski, I. Li, B. Howell, B. Priddy, Polym. Prepr. (Am. Chem. Soc. Div. Polym. Chem.) 1996, 37, 519

[60] G. Schmidt-Naake, M. Drache, C. Han, A. Koch, Dechema 1998, 134, 601

[61] T. Fukuda, T. Terauchi, Chem. Lett. (Tokyo) 1996, 293

[62] M. Buback, F.D. Kuchta, J. Macromol. Chem. Phys. 1997, 198, 1455

[63] T. Fukuda, T. Terauchi, T. Miyamoto, Polym. Prepr. Jpn. 1996, 45, 1261

[64] K. Matyjaszewski, D. Greszta, J. Polym. Sci. Polym. Chem. 1998, 29

[65] A. Goto, K. Ohno, T. Fukuda, Macromolecules 1998, 31, 2809

[66] A. Goto, K. Sato, T. Fukuda, G. Moad, E. Rizzardo, S. Thang, Polym. Prepr. (Div. Polym. Chem. Am. Chem. Soc.) 1999, 40 (2), 397

[67] K. Ohno, Y. Tsujii, T. Miyamoto, T. Fukuda, M. Goto, K. Kobayahi, T. Akaike, Macromolecules 1998, 31, 1064 
[68] T. Kothe, S. Marque, R. Martschke, M. Popov, H. Fischer, J. Chem. Soc., Perkin Trans. 1998, 2, 1553

[69] M. Busch, submitted to Macromol. Theory and Simul. 


\section{EXPERIMENTAL}

\subsection{Apparatus}

\subsubsection{Optical high-pressure cell}

The optical high-pressure cell used for spectroscopic investigations into chemically induced homo- and copolymerizations under high pressure is illustrated in Fig. 3.1. The cell is designed for pressures up to 3500 bar and temperatures up to $350^{\circ} \mathrm{C}$. The cylindrical cell body and sealing flanges are made of a nickel-based alloy of high ultimate tensile strength (RGT 601, Material No. 2.4668). The cell length is $100 \mathrm{~mm}$. The outer and inner diameters are 80 and $22 \mathrm{~mm}$, respectively. Four holes bored perpendicular to the cylindrical axis allow for fitting of high-pressure capillaries and insertion of a sheathed thermocouple (6) directly into the sample volume. Unused borings are closed with plugs. The cell is sealed at each end by a conical ram (5) (Material No. 2.4668) which fits into the cell cone. The ram is pressed into the cell cone by the flange (2) (Material No. 2.4668) which is secured by six high-pressure bolts (1) (Material No. 2.4969). It is the length of the rams which determines the optical path length. The rams were chosen such that a path length of approximately $1 \mathrm{~mm}$ resulted.

Each high-pressure window (4) is fitted against the polished surface of the ram and held in place by a stainless steel cap. To compensate for surface irregularities, an approximately $12 \mu \mathrm{m}$ thick teflon foil is placed between the polished surface of the window and the ram. This set-up is self-sealing under high pressure in accord with the Poulter principle ${ }^{1}$. The high-pressure optical windows used in this work for homopolymerizations were made from synthetic sapphire crystals (diameter $18 \mathrm{~mm}$, height $10 \mathrm{~mm}$ ). This material is transparent in the wavenumber range 2000 to $10000 \mathrm{~cm}^{-1}$. The high-pressure optical windows used for the peroxide decomposition study were made from polycrystalline silicon. This material is transparent in the wavenumber range from 1450 to $7000 \mathrm{~cm}^{-1}$ and so allows detection of the carbonyl stretching fundamentals of peroxides and their decomposition products. 


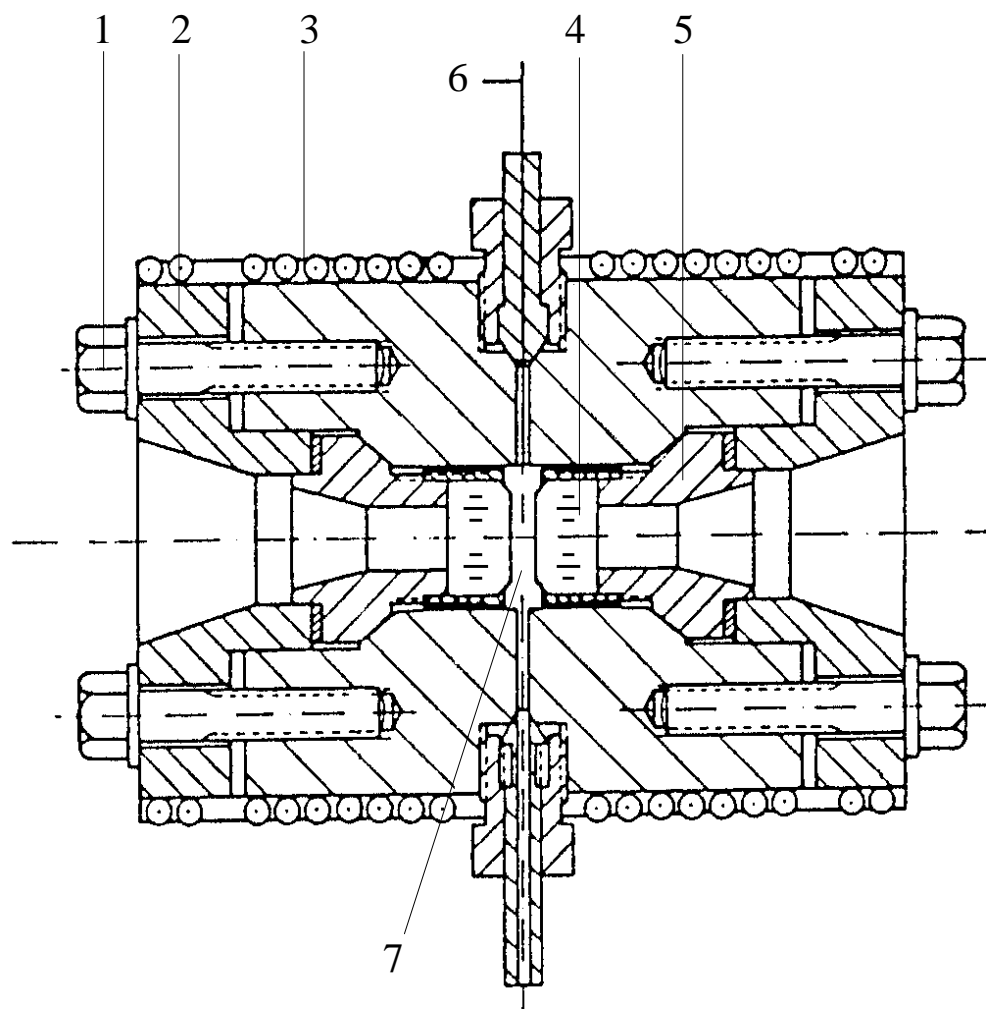
(1) bolt
(5) ram
(2) flange
(6) sheathed thermocouple
(3) heating jacket
(7) adjustable optic path length
(4) high-pressure window

Fig. 3.1. Optical high-pressure cell.

\subsubsection{Heating and temperature control}

Heating of the optical high-pressure cell is facilitated by two heating jackets. These consist of a brass matrix, into which is embedded a sheathed resistance heating wire (CGEAlsthom). The closely fitting jackets slide over each end of the cell body (see Fig. 3.1. (3)). The temperature is measured via a sheathed thermocouple (Nickel-chromium against nickel, CIA S250, CGE-Alsthom) and regulated by a PID-controller (Eurotherm 815). 


\subsubsection{FT-IR/NIR Spectrometer}

Infra red and near infra red spectra were recorded on a Bruker IFS-88 FourierTransform-Spectrometer. To house the heated optical high-pressure cell, the sample chamber of the spectrometer is enlarged (heightened) and fitted with a water cooled cell holder (to prevent heat transfer to the spectrometer). The chamber is purged with compressed air of low water and carbon dioxide concentration.

For the present work, several optical configurations have been used, depending on the system studied. These different configurations are reported for each set of experiments in Section 4.1 .

\subsubsection{Pressure generation and control}

For the kinetic investigations of ethene polymerization the system is pressurized using a high-pressure membrane pump (Nova) up to 1000 bar and a manually driven ("syringe pump“) pressure generator (volume $12 \mathrm{~cm}^{3}$ ) designed for pressures up to $3000 \mathrm{bar}$. The pressure is measured with a high-pressure precision manometer (Class 0.2, $0-3.5 \mathrm{kbar}$, Gefran).

In the continuous flow reactor, the reaction mixture is directly pressurized. Pressure is generated using a combination of an HPLC-pump (Pneumatic HPLC pump 70 000, Knauer) and a high-pressure membrane pump (MhR 150/7, Orlita). A DMS pressure transducer (Class $0.2,0-3 \mathrm{kbar}, \mathrm{HBM}-\mathrm{Me}$ (technik) is used to monitor the pressure. 


\subsection{Overall assembly}

\subsubsection{Continuous flow method}

The experimental design of the continuous flow reactor experiments is shown in Figure 3.2. This set-up has been used to study peroxide decomposition in the presence of nitroxide in a wide range of temperatures $\left(120-220^{\circ} \mathrm{C}\right)$ and pressures $(500-2500 \mathrm{bar})$.

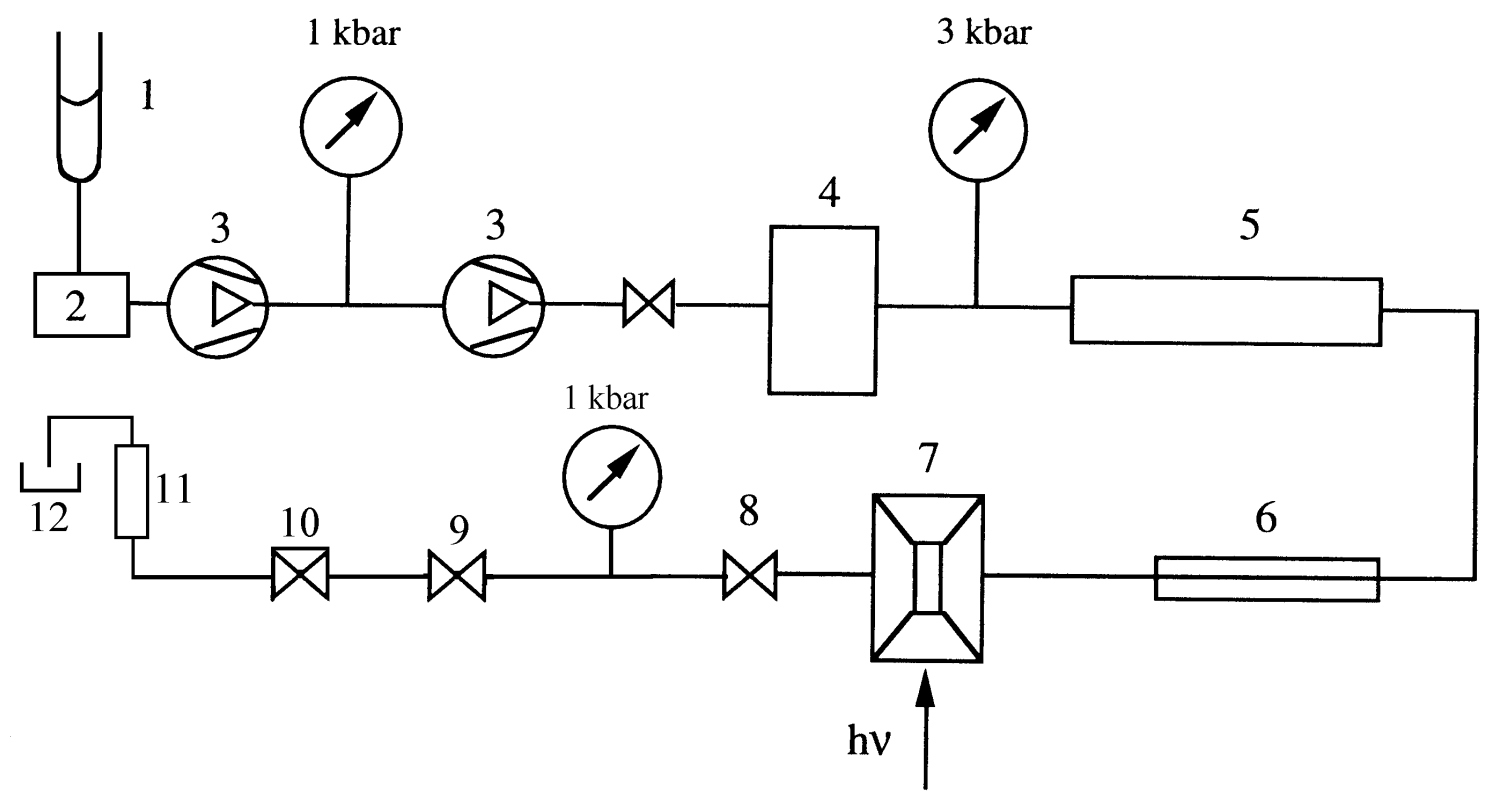
(1) peroxide reservoir
(7) optical high pressure cell
(2) HPLC degasser
(8) valve
(3) high-pressure pumps
(9) sample valve GC
(4) buffer volume
(10) low pressure valve
(5) tubular reactor
(11) flow meter
(6) cooler
(12) collector

Fig. 3.2. Schematic view of the apparatus used for investigations into peroxide decomposition in the continuous flow reactor (see text for explanation).

As described by Buback and Klingbeil ${ }^{2}$, the reaction medium is introduced into a reservoir (1). The solution is placed for $10 \mathrm{~min}$ in an ultrasonic wave bath for removal of air. The solution is fed through a HPLC degasser (2) from the reservoir to an HPLC pump (3), which pressurizes the medium up to 800 bar before feeding into the high-pressure membrane 
pump (3). After passing the two pumps, the solution enters a buffer volume of $20 \mathrm{ml}$ (4). The transfer tube between the buffer and the reactor is heated to a temperature of $50^{\circ} \mathrm{C}$. The tubular reactor (5) (inner diameter $0.5 \mathrm{~mm}$, length $10.0 \mathrm{~m}$ ) is placed in an oil bath which is held to the desired temperature within $\pm 0.1^{\circ} \mathrm{C}$. The pressure is measured at the entrance of the reactor. The transfer tube from the reactor to the IR cell is cooled to $50^{\circ} \mathrm{C}$ to quench the reaction and keep the solution homogeneous. The concentrations of educt or product are monitored in an optical high-pressure cell (7) which is heated to $50^{\circ} \mathrm{C}$ and held in the sample compartment of an FTIR spectrometer. The solution is then expanded (8) by a high-pressure fine valve, thermostated to $50^{\circ} \mathrm{C}$. The solution passes a sample loop of a GC sample valve (9). After passing the tubular reactor, the flow is measured by a calibrated flow meter (11) and collected (12).

\subsubsection{Styrene and (meth)acrylate polymerization}

The apparatus used for the kinetic experiments with liquid monomers such as styrene and (meth)acrylates under high pressure is schematically shown in Figure 3.3.

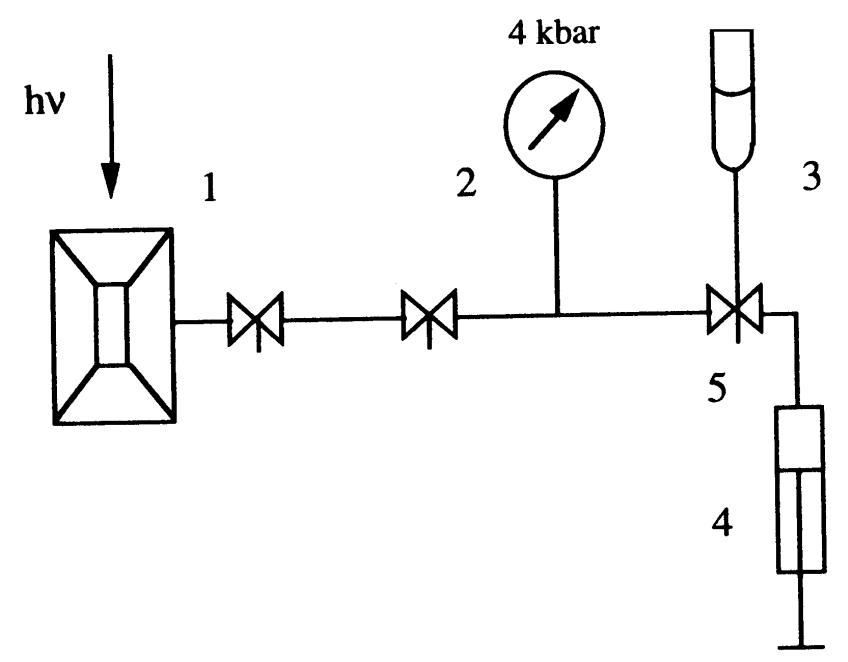

(1) optical high pressure cell

(4) "syringe" type pressure generator

(2) manometer

(5) valve

(3) reservoir for monomer

Fig. 3.3. Schematic view of the apparatus used for investigations into styrene and (meth)acrylate polymerizations. 
The monomer acts as the pressure-transmitting medium. The monomer reservoir is closed by a silicon septum. Monomer is introduced into the pressure generator through a fine capillary which passes through the silicon septum. Via the pressure generator, the monomer is pressed into the high-pressure cell up to the desired pressure. The optical cell is aligned into the sample chamber of the FTIR spectrometer.

\subsubsection{Ethene polymerization}

The apparatus used for the ethene polymerizations is shown in Figure 3.4.

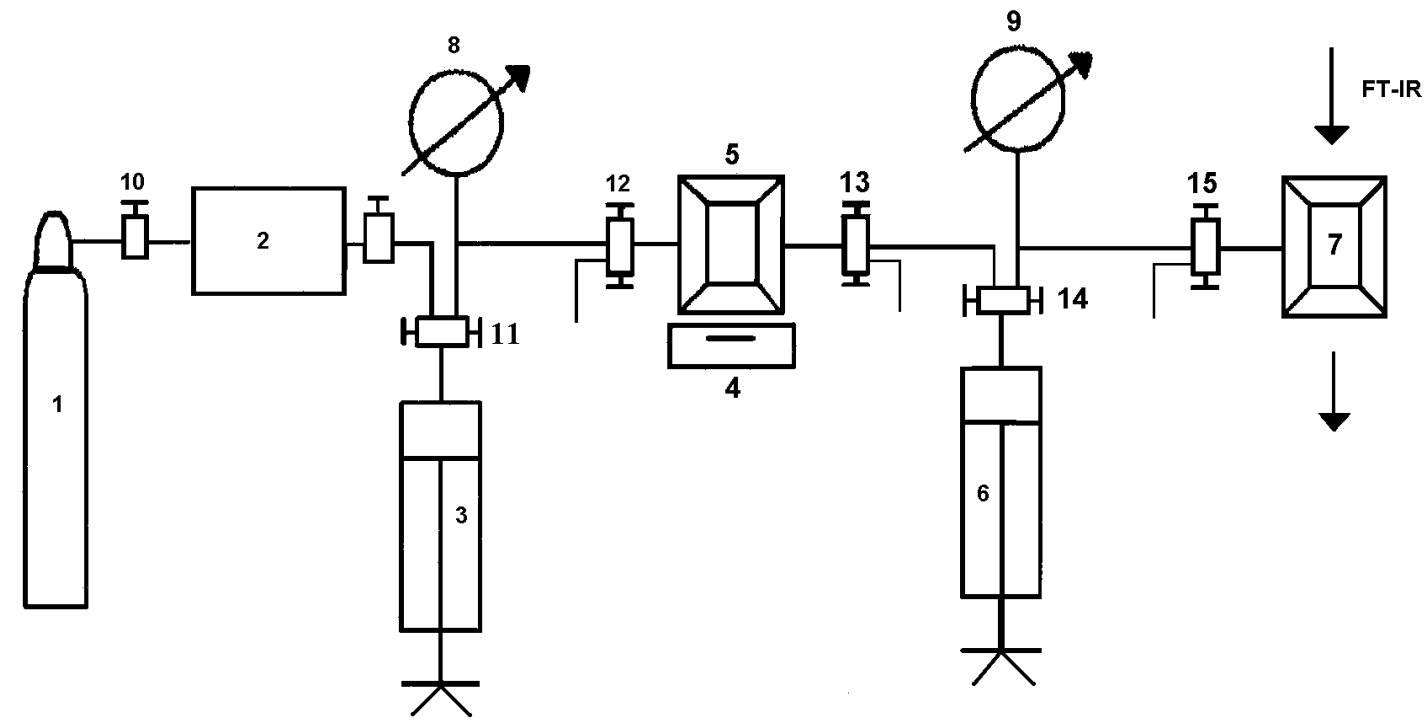

(1) ethene storage

(2) high-pressure membrane pump

$(3,6)$ "syringe" type pressure generator

(4) magnetic stirrer
(5) high-pressure mixing cell

(7) optical high-pressure cell

$(8,9)$ DMS transducers

(10-15) valves

Fig. 3.4. Schematic view of the apparatus used for investigations into ethene polymerization.

Ethene, at a pressure of about 60 bar is compressed with a membrane compressor (2) up to 1000 bar. Additional components may be added directly into the mixing cell (5). The 
mixture is compressed up to 2800 bar with the syringe pump (3). Mixture is stirred for ten minutes at room temperature and 2800 bar (or cooled to $0^{\circ} \mathrm{C}$ in the case of peroxides with low decomposition temperature). By opening valve 13 , the mixture is expanded into the optical cell and into the second syringe pump (6). Valve 13 is closed and the mixture is compressed until the desired pressure is reached. The optical cell (7) is positioned into the sample chamber of the FTIR spectrometer.

\subsection{SEC-experiments}

The molecular weight distribution of polyethylene samples have kindly been performed by ATOFINA, using a high-temperature SEC set-up operated with 1,2,4trichlorobenzene $(\mathrm{TCB})$ at $145^{\circ} \mathrm{C}$, using a Mixed B column (Polymer Laboratories, Church Stretton), Waters $150 \mathrm{CV}$ refractive and viscosimetric index detector (Millford. MA.). 14 PS (TSK, Tosoh Haas) standards have been used for the calibration of polymer molecular weights.

The molecular weight distributions of polystyrene and poly(meth)acrylate samples were determined by size-exclusion chromatography (SEC) ${ }^{3}$ using a Waters 515 pump, Waters 410 refractive index detector, PSS-SDV columns with nominal pore sizes of $10^{5}, 10^{3}$ and $10^{2} \AA$ And tetrahydrofuran (THF) at $30^{\circ} \mathrm{C}$ as the eluent. Molecular weight calibrations for the homopolymerization of styrene and MMA were established against narrow polydispersity PS $\left(M_{\mathrm{W}}\right.$ between 410 and $2 \cdot 10^{6} \mathrm{~g} \cdot \mathrm{mol}^{-1}$ ) and PMMA standards $\left(M_{\mathrm{W}}\right.$ between 670 and $\left.2.4 \cdot 10^{6} \mathrm{~g} \cdot \mathrm{mol}^{-1}\right)$, respectively.

\subsection{Simulation tool PREDICI ${ }^{\circledR}$}

The kinetic models described in this work are implemented in the simulation program PREDICI $^{\circledR}$ (Polyreaction Distributions by Countable System Integration), developed by M. Wulkow ${ }^{4-6}$. The program uses a highly efficient algorithm, called discrete hp-algorithm for solving complex sets of countable differential equations. An integrated function interpreter enables the coupling of rate coefficients of elementary reaction steps with individual species. 
As a special feature of PREDICI ${ }^{\circledR}$ is that the full molecular weight distribution MWD may be simulated without any assumptions of closure conditions.

\subsection{Chemicals}

\subsubsection{Nitroxides and alkoxyamines}

Nitroxides and alkoxyamines have been used as received, without further purification. Samples were stored at $3^{\circ} \mathrm{C}$. Different nitroxides have been used. Only a few of them are commercially available. The other materials have been provided by organic chemistry laboratories, SG1 and derivatives by Professor Tordo (University of Marseille) and DPAIO and derivatives by Professor Greci (University of Ancona). The structures of these materials are reported in Figure 3.6.

\begin{tabular}{lccc}
\hline chemicals & abbreviation & purity & manufacturer \\
\hline di-tert-butylnitroxide & DTBN & $97 \%$ & Lancaster \\
$n$-tert-butyl-1-diethylphospono-2,2- & SG1 (or DEPN) & $82 \%$ & University of \\
dimethylpropyl nitroxide & & & Marseille \\
2,2,6,6-tetramethyl-1-piperidinyloxyl & TEMPO & $98 \%$ & Aldrich \\
3-imino-2-phenyl indolinonic nitroxide & DPAIO & $100 \%$ & University of \\
& & & Ancona \\
CXA & CXA & $98 \%$ & ATOFINA
\end{tabular}

Table 3.5. $\quad$ Nitroxides used in this work.<smiles>CC1(C)CCCC(C)(C)N1O</smiles>

TEMPO<smiles>CC(C)(C)N([O])C(C)(C)C</smiles>

DTBN<smiles>CCOP(C([O])N([O])CC)C(C(C)(C)C)C(C)(C)C</smiles>

SG1 (or DEPN)<smiles>CN1c2ccccc2/C(=N/c2ccccc2)C1(c1ccccc1)c1ccccc1</smiles>

DPAIO

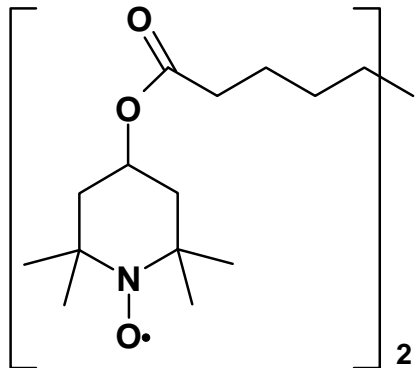

CXA

Fig. 3.6. $\quad$ Structures of the nitroxides used in this work. 
As alkoxyamine initiators the associated nitroxide-capped styryl compounds S-SG1, S-TEMPO, S-DPAIO and S-DTBN (see Fig. 3.7.) have also been used. They were provided by Professor Tordo's and Greci's laboratories.<smiles>CC(ON1C(C)(C)CCCC1(C)C)c1ccccc1</smiles>

S-TEMPO<smiles>CC(ON(C(C)(C)C)C(C)(C)C)c1ccccc1</smiles>

S-DTBN<smiles>CCOP(=O)(OCC)C(N(OC(C)c1ccccc1)C(C)(C)C)C(C)(C)C</smiles>

S-SG1<smiles>CC(ON1c2ccccc2C(=Nc2ccccc2)C1(c1ccccc1)c1ccccc1)c1ccccc1</smiles>

S-DPAIO

Fig. 3.7. $\quad$ Structures of the alkoxyamines used in this work.

\subsubsection{Peroxides}

For kinetic investigations of peroxide decomposition in the presence of nitroxide, three peroxides have been used. They were provided by AKZO NOBEL. The decomposition of these peroxides follows a first-order rate law. They were chosen such as to allow for peroxide decomposition in a wide range of temperatures (see table 3.8). For the experiments carried out in the continuous flow reactor with $n$-heptane as solvent, $0.01 \mathrm{M}$ solutions of TAPP and TBPA and a 0.1 M solution for DTBP have been used. These peroxides were used without further purifications.

\begin{tabular}{ccccc}
\hline chemical & abbreviation & purity & structure & temperature range \\
\hline tert-amylperpivalate & TAPP & $97.1 \%$ & perester & $120-140^{\circ} \mathrm{C}$ \\
di-tert-butylperoxide & DTBP & $99 \%$ & diacyl peroxide & $180-220^{\circ} \mathrm{C}$ \\
tert-butylperacetate & TBPA & $94.6 \%$ & peracetate & $160-180{ }^{\circ} \mathrm{C}$ \\
\hline
\end{tabular}

Table 3.8. $\quad$ Peroxides used in the studies of peroxide decomposition in the presence of nitroxide. 
AIBN (Aldrich, 99.5\%) and BPO (Aldrich, $25 \%$ in water) have been used for chemically-initiated polymerizations in the presence of a nitroxide. Azo-bis isobutyronitrile (AIBN) is recristallized twice from $n$-hexane prior to use. Dibenzoylperoxide (BPO) was supplied stabilized by water. Aliquots were added to water, passed through a sintered glass filter, and treated by a series a water-methanol-water rinsings before being drying, first in a cupboard overnight and then in a dessicator for several days, following the procedure recommended by Gerritsen ${ }^{7}$.

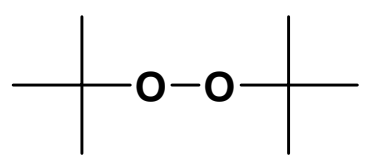

DTBP

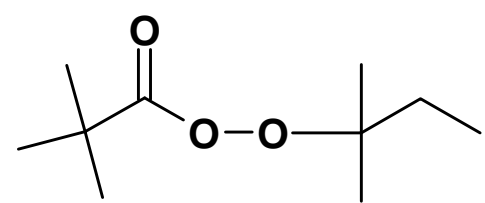

TAPP

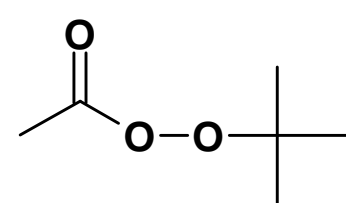

TBPA

Fig. 3.9. $\quad$ Structures of the peroxides used in this work.

\subsubsection{Monomers}

High purity ethene, E, $\left(\mathrm{O}_{2}<5 \mathrm{ppm}\right)$ was provided by BASF AG (Ludwigshafen, Germany) and by DSM (Geelen, Netherlands).

Styrene, S, (Fluka, $99 \%$, stabilized by $0.0005 \%$ 4-tert-butylcatechol) was washed several times with aqueous $\mathrm{NaOH}$ and with water to remove the inhibitor, then dried with $\mathrm{Na}_{2} \mathrm{SO}_{4}$ and distilled (using a Vigreux column) under reduced pressure $\left(\approx 45^{\circ} \mathrm{C}, 40\right.$ mbar). Great care has to be taken to avoid contamination of the monomer by vacuum grease. Therefore the distillation apparatus is kept sealed by teflon washers.

The acrylates $\left(\mathrm{R}_{2}=\mathrm{H}\right)$ and methacrylates $\left(\mathrm{R}_{2}=\right.$ Methyl $)$ have the following general structure :

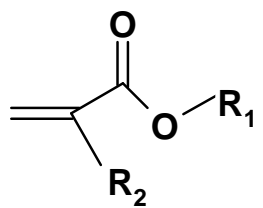

Methyl methacrylate, $\mathrm{R}_{1}=\mathrm{CH}_{3}$, MMA, (Fluka, purum $99.5 \%$, stabilized by $0.02 \%$ hydroquinone monoethylester) is distilled under reduced pressure $\left(\approx 47^{\circ} \mathrm{C}, 150 \mathrm{mbar}\right)$ in the presence of $\mathrm{K}_{2} \mathrm{CO}_{3}$ to remove the stabilizer. The purity of MMA is checked by recording a 
UV-spectrum at $351 \mathrm{~nm}$. The molar absorption coefficient should be below $3 \mathrm{~cm}^{3} / \mathrm{mol}$. Butyl acrylate, $\mathrm{R}_{1}=\mathrm{C}_{4} \mathrm{H}_{7}$, (BA) (Fluka, purum $99.5 \%$, stabilized by $0.02 \%$ hydroquinone monoethylester), is purified in the same way $\left(\approx 47^{\circ} \mathrm{C}, 50 \mathrm{mbar}\right)$. The purity of the monomer is checked by UV-spectrometry. The molar absorption coefficient should be below $5 \mathrm{~cm}^{3} / \mathrm{mol}$.

\subsubsection{Solvents}

The solvents used for the study of peroxide decomposition and for the polymerizations were obtained in high purity and were used without further purification : n-heptane (Fluka, $>99 \%$ ), toluene (Fluka, > $99.5 \%$ ). A mixture of methanol (Merck, $99.8 \%$ ) with hydroquinone (Merck, > $99 \%$ ) has been used to precipitate polymers and to quench styrene and (meth)acrylate polymerizations.

\subsection{Performance of a typical experiment}

The three different types of experiments performed within this work will now be described.

\subsubsection{Continuous flow method - peroxide decomposition}

For the preparation of the peroxide mixture, a two liter reservoir is filled with about $500 \mathrm{ml}$ solvent. The peroxide is added and then the remaining solvent. The solution is placed for $10 \mathrm{~min}$ in an ultrasonic bath for removal of air. The overall set-up, with the reactor at room temperature, is purged with the peroxide solution until a constant absorbance of the peroxide is observed in the carbonyl region. 200 up to $300 \mathrm{ml}$ solution are used for this step. Then the apparatus is further purged with $100 \mathrm{ml}$ of the mixture and at the end of the purge, two spectra of the peroxide solution are taken at the pressure selected for the experiments. The oil bath can now be heated to the desired temperature. The high-pressure pumps are switched off. With the high-pressure membrane pump and the GC sample valve, at least 5 up to 10 different residence time were chosen at constant temperature and pressure. Residence time was determined by measuring the flow with a calibrated flow meter. As soon as constant flow 
conditions are reached at each residence time, 2 spectra were taken at $30 \mathrm{~s}$ interval. While spectra are registered, the volume flow of the solution is measured downstream with a chronograph and a $10 \mathrm{ml}$ graduated cylinder at normal pressure. From the known $p V T$ data of the solvent and the well-known volume of the tubular reactor, the residence time is calculated in order to check the calibration of the flow meter.

1.51 peroxide solution allows for measurements of a sufficient number of different residence times at 7 to 9 temperature and pressure conditions. 150 up to $200 \mathrm{ml}$ peroxide solution are needed for the calculation of an individual decomposition rate coefficient.

\subsubsection{Styrene and (meth)acrylate polymerization}

The stock solutions (peroxides, nitroxides, and monomers), are removed from the freezer or the fridge where they are stored, and allowed to warm up to room temperature. Compounds are weighted into a round bottom flask which is fitted with a tap and adaptator for the high-vacuum pump. The reaction mixture is then treated to a series of freeze-pump-thaw cycles using liquid nitrogen and a high-vacuum molecular drag pump (Drytel 26, Alcatel). Typically 4 freeze-pump-thaw cycles were performed. During this time, the tightness of the optical cell has to be checked by pressurizing it at the desired pressure with carbon dioxide. The cell is then placed in the sample compartment of the spectrometer. The temperature regulator is connected to the thermocouple and the cell is heated up to the desired temperature. Oxygen is removed by a vigorous flow of nitrogen through the set-up for $\approx 15$ $\min$.

The round bottom flask, together with a cap closed by a septum, is then introduced into the evacuation chamber of an inert gas glove box. The chamber is evacuated for at least 10 min. During this time the reference spectrum of the empty optical cell at the desired temperature is recorded in the region $1500-7000 \mathrm{~cm}^{-1}$ by co-addition of 100 scans.

In the glove box, the tap is removed from the flask and is closed with a septum. The flask is then removed from the glove box. The nitrogen flow in the high-pressure set-up is then reduced. The set-up is connected to the flask by introducing a thin metal capillary through the septum. The nitrogen flow is then stopped. The solution is drawn into the syringe pump. If the reaction temperature is reached, the solution is pressed into the optical high- 
pressure cell to the desired pressure. As soon as temperature and pressure have equilibrated, the collection of spectra is started. Typically, 100 scans were taken for each interferogram. Styrene concentration is then followed on line during the course of the polymerization. When the desired conversion is reached, the solution is collected and precipitated in a mixture of methanol-hydroquinone.

\subsubsection{Ethene polymerization}

The nitroxide and peroxide containing mixture is prepared as described in section 3.7.2. Only the set-up is different. The tightness of the optical cell has to be checked by pressurizing it to the desired pressure with carbon dioxide. Then the empty optical cell is placed in the sample compartment of the spectrometer and connected to the pressuregenerating system via a metal capillary. The temperature regulator is connected to the thermocouple and the cell is heated via two heating jackets. Oxygen is removed by purging the internal volume with ethene, six times at bottle pressure $(\approx 60 \mathrm{bar})$, four times at $1000 \mathrm{bar}$ and four times at 2000 bar. The outlet valves are regulated such as to have a pressure of $10 \mathrm{bar}$ in the mixing cell and of 2 to 3 bar in the optical cell. The flange of the mixing cell is then opened and valve 12 is regulated so that a weak ethene flow avoids oxygen contamination.

The flask, closed with a septum, is removed from the glove box. The solution is drawn with a syringe through the septum and introduced into the mixing cell. The mixing cell is closed and compressed to 2800 bar with ethene via the membrane compressor and the syringe pump. The mixture is stirred for $10 \mathrm{~min}$ at room temperature. During this time the reference spectrum of the empty heated optical cell is taken over the region $1500-10000 \mathrm{~cm}^{-1}$ and generated from a co-addition of 100 scans. The solution is then driven into the second syringe pump at a pressure of about 250 bar. As soon as the reaction temperature is reached, the solution is driven into the optical cell and the pressure is raised to that desired value. As soon as temperature and pressure have equilibrated, the collection of spectra is started. After reaching the desired conversion, pressure is carefully released by very slowly opening valve 15. The IR high-pressure cell is then removed from the IR spectrometer and cooled down with a ventilator. At $50^{\circ} \mathrm{C}$, the cell is opened and polyethylene is collected. 


\subsection{Error estimate}

The reaction temperature is accurate within $\pm 0.5 \mathrm{~K}$ with the uncertainty resulting from the thermocouple and the PID controller.

The accuracy of the pressure measurement ('HBM-Meßtechnik', Class 2, maximal pressure 3000 bar) is $\pm 0.2 \%$ of the scale limit, resulting in an error of \pm 6 bar.

Quantitative measurements with the Fourier-Transform-Spectrometer can be achieved at absorbances in the range 0.02 and 0.8 . The lower boundary is determined by the signal-tonoise ratio, and the upper limit by non-linearity of the detector. For absorbances between 0.3 and 0.8 , the non-linear behavior of the detector causes an error of less than $1 \%$. The uncertainty in the determination of the base line results in an error in the integrated absorbance (required for quantitative evaluations) of less than $\pm 3 \%$ at maximum absorbance up to 0.5 . Due to the uncertainty in the position of the maximum, this error is raised to $\pm 5 \%$ in determination of half-band integrals. The relative error in integrated absorbance results in a maximum error of $\pm 2 \%$ for the kinetic evaluation.

For the preparation of the mixtures, an analysis balance (Sartorius) was used which allows measurements to 4 decimal places. The absolute error should be $\pm 0.1 \mathrm{mg}$. However, the actual error in the concentration is larger, as the balance is very sensitive to small disturbances. The concentration should, however, be accurate within $\pm 2 \%$.

\subsection{References}

[1] T. C. Poulter, Phys. Rev. 1932, 40, 860

[2] M. Buback, S. Klingbeil, Chem. Ing. Tech. 1995, 67, 493

[3] O. F. Olaj, I. Bitai, F. Hinkelmann, Makromol. Chem. 1987, 188, 1689

[4] P. Deuflhard, M. Wulkow, Konrad Zuse Zentrum, Berlin, Preprint SC94-22 1994 
[5] "Predici - the software package for realizing polymerization kinetics", proceedings of the 8th conference of the european consortium of mathematics in industry 1994 , Springer, Berlin 1995

[6] M. Wulkow, Macromol. Theory Simul. 1996, 2, 393

[7] R. Gerritsen, Private communications 1994 


\section{KINETIC MODELS AND THEORETICAL PREDICTIONS}

Simulations are now frequently used for analyzing and understanding complex chemical reactions such as the high-pressure fluid phase polymerization. The qualitative and quantitative description of styrene and ethene polymerizations in the presence of a nitroxide or an alkoxyamine requires the interpretation of a complex reaction with simultaneous consideration of kinetic information deduced from independent experiments. Computer simulations are also helpful for the development of the experimental strategy. The remarkable progress in this field during recent years is essentially due to the availability of efficient mathematical tools such as the program package PREDICI ${ }^{\circledR}$.

\subsection{Kinetic model for controlled styrene homopolymerization. \\ General description and development of a strategy for parameter estimations}

Schmidt-Naake et al. presented a model ${ }^{1}$ for the N-oxyl controlled radical polymerization of styrene and applied it to experimental data in which the $\mathrm{N}$-oxyl concentration has been varied. This model adequately describes the kinetics of polymerization reaction at ambient pressure, where nitroxide has been used together with an initiator such as dibenzoyl peroxide BPO. This model will now be modified for controlled radical polymerization initiated by alkoxyamines. Finally, the model will be extended to simulations of controlled radical polymerization at high pressure. Well known and also estimated activation volumes of the individual kinetic steps will be introduced for this purpose.

\subsubsection{Elementary reactions in nitroxide-mediated polymerization of styrene}

In the present work, both types of initiating systems, nitroxide plus initiator and alkoxyamine, will be used. Initiators usually used are dibenzoyl peroxide, dicumyl peroxide or azo-bis-isobutyronitrile in the presence of a nitroxide such as TEMPO $(2,2,6,6$, 
tetramethylpiperidinyl-1-oxy). For the simulations and in the experiments, polymerizations will be preferably initiated with a $\mathrm{N}$-oxyl-adduct or an alkoxyamine : in these cases, no excess of nitroxide is used which permits to reach the steady-state more rapidly. In fact, it was already shown ${ }^{3}$ on the basis of experimental and simulated data for a TEMPO-styrene system, that the stationary state holds accurately except for a short initial period. Otherwise, the rate of polymerization of the stable free-radical polymerization process was shown to be independent of the amount of initiator ${ }^{4}$ and, instead dependent on the concentration of excess nitroxide.

Generally, the N-oxyl or alkoxyamines containing system at high temperature may involve the following elementary reaction steps with the associated rate coefficients.

$$
\begin{aligned}
& I \stackrel{k_{\mathrm{I}}}{\longrightarrow} 2 I^{\bullet} \\
& \text { (1) initiation by a system initiator+nitroxide } \\
& I^{\bullet}+M \stackrel{k_{\mathrm{p}}}{\longrightarrow} R_{1} \\
& R_{1}+N \stackrel{k_{\mathrm{c}} / k_{\mathrm{d}}}{\longrightarrow} R_{1} N \\
& R_{1} N \stackrel{k_{\mathrm{d}} / k_{\mathrm{c}}}{\longleftrightarrow} R_{1}+N \quad \text { (2) initiation by an alkoxyamine } \\
& 3 M \stackrel{k_{\mathrm{i}}}{\longrightarrow} 2 R_{1} \quad \text { (3) spontaneous styrene thermal initiation } \\
& R_{n}+M \stackrel{k_{\mathrm{p}}}{\longrightarrow} R_{n+1} \quad \text { (4) propagation } \\
& R_{n} N \stackrel{k_{\mathrm{d}}}{\longrightarrow} R_{n}+N \quad \text { (5) dissociation of the dormant species } \\
& R_{n}+N \stackrel{k_{\mathrm{c}}}{\longrightarrow} R_{n} N \\
& R_{n}+R_{m} \stackrel{k_{\mathrm{t}}}{\longrightarrow} P_{n+m} \text { or } P_{n}+P_{m} \quad \text { (7) termination } \\
& R_{n}+M \stackrel{k_{\mathrm{tr}}}{\longrightarrow} P_{n}+R_{1} \quad \text { (8) chain transfer } \\
& R_{n} N \stackrel{k_{\text {decomp }}}{\longrightarrow} P+N H \quad \text { (9) irreversible decomposition of the alkoxyamine } \\
& 2 N \stackrel{k_{\mathrm{dN}}}{\longrightarrow} \mathrm{NH}+\mathrm{N}^{*} \quad \text { (10) thermal decomposition of the nitroxide }
\end{aligned}
$$

Fig. 4.1. Elementary reactions in a nitroxide-mediated polymerization of styrene 
where $R_{n}$ is the growing radical, $P_{n}$ is the dead polymer, $M$ is the monomer, $I$ is the initiator, $N$ is the persistent radical, $\mathrm{R}_{\mathrm{n}} \mathrm{N}$ is the dormant species, $\mathrm{NH}$ and $\mathrm{N}^{*}$ are non reactive species issued from the decomposition of the nitroxide and of the dormant species.

The spontaneous thermal initiation of styrene has been found to be a third order reaction in the monomer ${ }^{7}$ concentration, with a rate coefficient $k_{\mathrm{i}}$. In the presence of a radical initiator, the two initiations are additive. The rate constants of propagation, termination and transfer to monomer of styrene polymerization are available in the literature ${ }^{1,7,8}$. Chain transfer to monomer is assumed to be far less important than transfer to the Diels-Alder adduct or to the Mayo dimer ${ }^{2,7}$.

The decomposition of the adduct polystyrene-TEMPO (PS-TEMPO) at high temperature through abstraction of the $\beta$-proton by the TEMPO, producing an alkene and a hydroxylamine (Fig. 4.2), has been reported by Li et al. ${ }^{9}$. First order decomposition kinetic was confirmed and rate constant $k_{\mathrm{decomp}}$ was reported ${ }^{3}$. In this model, $k_{\mathrm{d}}$ and $k_{\mathrm{c}}$ are assumed not to be chain length dependent.

(a)<smiles>CC(C)CC(C)c1ccccc1/C=C\c1ccccc1</smiles>

(b)

2<smiles>CC1(C)CCCC(C)(C)N1[O]</smiles><smiles>CC#N</smiles><smiles>CC1(C)CCCC(C)(C)N1O</smiles><smiles>CC(C)=CCC=C(C)C</smiles>

Fig. 4.2. Irreversible decomposition of PS-TEMPO through $\beta$-proton abstraction (a) and irreversible thermal decomposition of TEMPO $(b)$

The dissociation and combination rate coefficients for the controlled styrene-TEMPO system have been reported by Schmidt-Nake et al. ${ }^{1}$. 
It has been suggested that degenerative transfer may participate in the activation process of the styrene/TEMPO system ${ }^{5-6}$, but no clear evidence has been provided so far. Thus the simulation model will not take this phenomenon into account.

\subsubsection{Pressure dependence of TEMPO-mediated styrene polymerization}

In this section, mechanism and kinetics of the TEMPO-mediated styrene polymerization at $125^{\circ} \mathrm{C}$ and pressures up to 2000 bar are discussed with respect to both experimental and simulated data.

The effects of high pressure on initiator efficiencies, propagation and termination rate coefficients in the free-radical bulk polymerization of styrene over an extended temperature and pressure range have been reported by Buback et al. ${ }^{10-12}$. It has been shown that the efficiency of AIBN decreases with pressure and conversion. Activation volumes for the propagation and termination rate coefficients have been calculated in low conversion styrene bulk polymerization up to $90^{\circ} \mathrm{C}$ and 2000 bar. The data which is given in Tab. 4.3. may be linearly extrapolated to $125^{\circ} \mathrm{C}$.

\begin{tabular}{lcc}
\hline$\theta /{ }^{\circ} \mathrm{C}$ & $\Delta V^{\ddagger}\left(k_{\mathrm{p}}\right)$ & $\Delta V^{\neq}\left(k_{\mathrm{t}}\right)$ \\
& $\mathrm{cm}^{3} \cdot \mathrm{mol}^{-1}$ & $\mathrm{~cm}^{3} \cdot \mathrm{mol}^{-1}$ \\
\hline 30 & $-12.1 \pm 1.1$ & $13.6 \pm 2.6$ \\
50 & $-11.7 \pm 0.8$ & $15.4 \pm 1.6$ \\
70 & $-11.4 \pm 1.1$ & $17.1 \pm 2.2$ \\
90 & $-11.1 \pm 0.8$ & $18.9 \pm 2.2$ \\
125 (extrapolated) & $-10.5 \pm 1.1$ & $22.0 \pm 2.2$ \\
\hline
\end{tabular}

Tab. 4.3. Activation volumes of propagation and termination rate coefficients in low conversion styrene, obtained from linear fits of the isotherms 30, 50, 70 and $90^{\circ} \mathrm{C}$ and extrapolated to $125^{\circ} \mathrm{C}$.

Activation volume data for the reversible deactivation-activation reactions of the growing radical are not known and need to be estimated. 
The $\mathrm{N}-$ oxyl and alkyl radicals produced in the initiation step differ strongly in their reactivity. It is assumed that the pressure influence should be similar in the case of the combination of an $\mathrm{N}$-oxyl radical with an alkyl radical and for the termination reaction (by recombination) of two reactive alkyl radicals. The activation volume of $k_{\mathrm{t}}$ at $125^{\circ} \mathrm{C}$ is extrapolated from the experimental data in Tab. 4.3 to be $22 \pm 2.2 \mathrm{~cm}^{3} \cdot \mathrm{mol}^{-1}$.

It is further wise assumed that the pressure dependence should not strongly differ for the dissociation of the dormant species and of a peroxide dissociation. The activation volume for the homolytic decomposition of benzoyl peroxide, $\Delta V^{\neq}{ }_{\text {ВPO }}=12.1 \mathrm{~cm}^{3} \cdot \mathrm{mol}^{-1}$, is used to estimate the pressure dependence of dissociation of the dormant species.

Experimental investigations of TEMPO thermal decomposition, characterized by the rate coefficient $k_{\mathrm{dN}}$, under high pressure showed no pressure-induced TEMPO decomposition. It is also assumed that the decomposition of the adduct PS-TEMPO by abstraction of a $\beta-$ proton $\left(k_{\text {decomp }}\right)$ will not be influenced by pressure.

The rate coefficients of the model are summarized in Tab. 4.4.

$\begin{array}{llll}k_{\mathrm{I}} & = & 4.39 \cdot 10^{16} \exp \left(-19030 \cdot(T / \mathrm{K})^{-1}-0.145 \cdot(P / \mathrm{bar}) \cdot(T / \mathrm{K})^{-1}\right) & \mathrm{s} \\ k_{\mathrm{p}}= & 3.47 \cdot 10^{7} \exp \left(-4100 \cdot(T / \mathrm{K})^{-1}+0.126 \cdot(P / \mathrm{bar}) \cdot(T / \mathrm{K})^{-1}\right) & \mathrm{L} \cdot \mathrm{mol}^{-1} \cdot \mathrm{s}^{-1} \\ k_{\mathrm{t}}= & 1.25 \cdot 10^{9} \exp \left(-844 \cdot(T / \mathrm{K})^{-1}-0.265 \cdot(P / \mathrm{bar}) \cdot(T / \mathrm{K})^{-1}\right) & \mathrm{L} \cdot \mathrm{mol}^{-1} \cdot \mathrm{s}^{-1} \\ k_{\mathrm{c}}= & 7.11 \cdot 10^{6} \exp \left(-824 \cdot(T / \mathrm{K})^{-1}-0.265 \cdot(P / \mathrm{bar}) \cdot(T / \mathrm{K})^{-1}\right) & \mathrm{L} \cdot \mathrm{mol}^{-1} \cdot \mathrm{s}^{-1} \\ k_{\mathrm{d}}= & 2.00 \cdot 10^{13} \exp \left(-14940 \cdot(T / \mathrm{K})^{-1}-0.145 \cdot(P / \mathrm{bar}) \cdot(T / \mathrm{K})^{-1}\right) & \mathrm{s} \\ k_{\mathrm{tr}}= & 6.90 \cdot .10^{6} \exp \left(-6377 \cdot(T / \mathrm{K})^{-1}\right) & \mathrm{L} \cdot \mathrm{mol}^{-1} \cdot \mathrm{s}^{-1} \\ k_{\mathrm{decomp}}= & 5.80 \cdot 10^{14} \exp \left(-18430 \cdot(T / \mathrm{K})^{-1}\right) & \mathrm{L} \cdot \mathrm{mol}^{-1} \cdot \mathrm{s}^{-1} \\ k_{\mathrm{dN}}= & 3.80 \cdot 10^{14} \exp \left(-18430 \cdot(T / \mathrm{K})^{-1}\right) & \mathrm{L} \cdot \mathrm{mol}^{-1} \cdot \mathrm{s}^{-1} \\ k_{\mathrm{i}} & = & 1.32 \cdot 10^{-10} \quad \text { estimated by experimental data fit } & \mathrm{s}\end{array}$

Tab. 4.4. Constants used for controlled radical homopolymerization of styrene

Simulations using these coefficients and rate laws have been carried out using the PREDICI $^{\circledR}$ program. An ideal stirred batch reactor is used as the reactor model. Ideal micromixing is assumed. 


\subsubsection{Spontaneous styrene initiation}

As no rate coefficient for spontaneous styrene initiation for $125^{\circ} \mathrm{C}$ and 1 bar is available in the literature, $k_{\mathrm{i}}$ has been estimated from an experiment at ambient pressure and $125^{\circ} \mathrm{C}$ in which $14 \%$ styrene conversion were reached after 3 hours. $k_{\mathrm{i}}$ is obtained to be $1.32 \cdot 10^{-10} \mathrm{~s}^{-1}$. The simulated molar masses are independent of conversion and are typical for a free-radical polymerization process. Table 4.5 summarizes the experimental and simulated molar masses for different conversion.

\begin{tabular}{cccc}
\hline conversion $/ \%$ & time $/$ min & exp. $M_{\mathrm{N}} / \mathrm{g} \cdot \mathrm{mol}^{-1}$ & simul. $M_{\mathrm{N}} / \mathrm{g} \cdot \mathrm{mol}^{-1}$ \\
\hline 4.6 & 60 & 177000 & 199000 \\
8.9 & 120 & 200000 & 199000 \\
14.0 & 180 & 175000 & 199000 \\
\hline
\end{tabular}

Tab.4.5. Experimental and simulated number average molecular weights for spontaneous styrene polymerizations at $125^{\circ} \mathrm{C}$ and 1 bar.

The molar mass distributions of the polymer obtained in reactions to different degrees of monomer conversion are plotted in Fig. 4.6. The good agreement between experimental and simulated molar mass distributions shows that the model is capable of satisfactorily describing the spontaneous styrene polymerization at $125^{\circ} \mathrm{C}$ and 1 bar.

The experimental and simulated kinetics for the spontaneous polymerization of styrene at ambient pressure and at 2000 bar, plotted in Fig. 4.7, show a significant increase of polymerization rate at high pressure. A close agreement between calculated and measured data is observed, without significant pressure dependence of the rate coefficient $k_{\mathrm{i}}$ for spontaneous styrene polymerization. 


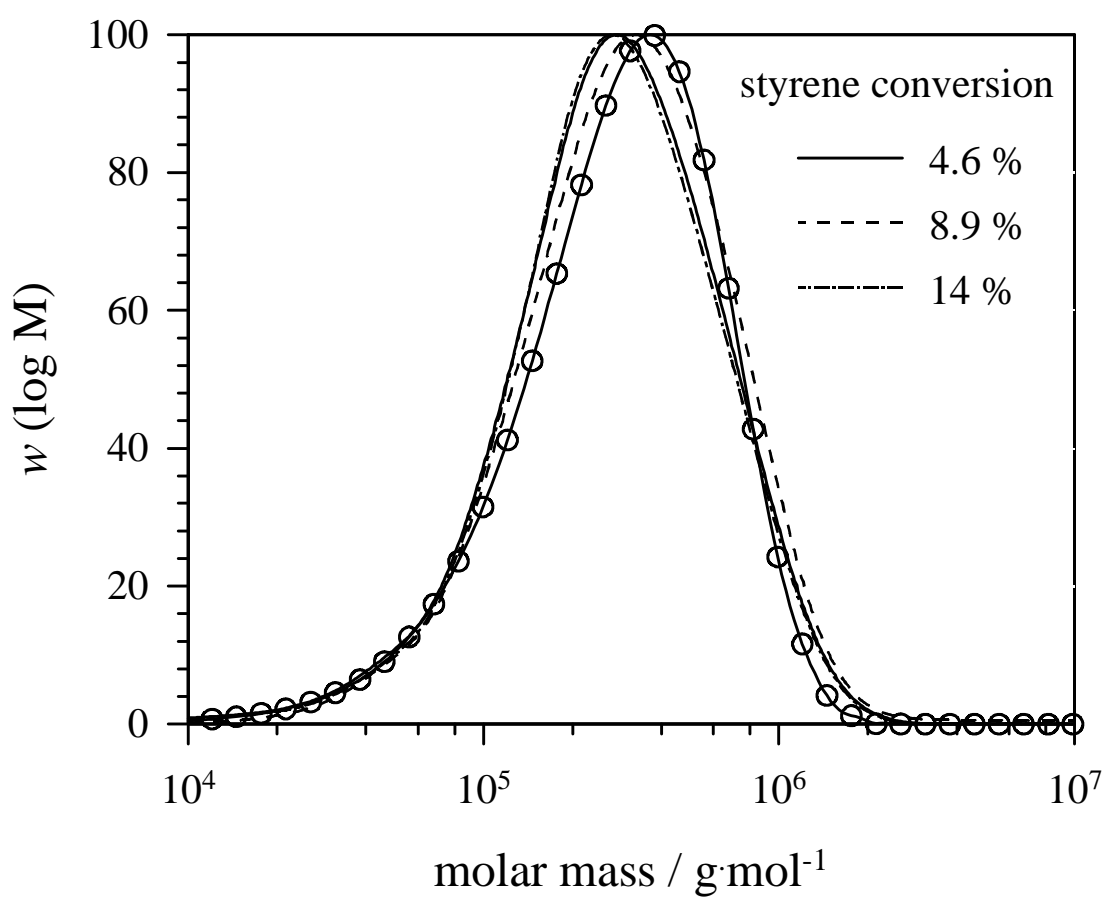

Fig. 4.6. Comparison of experimental molecular weight distributions (lines) at different degrees of monomer conversion with a simulated MWD (circles) at $14 \%$ conversion. All data refer to spontaneous styrene polymerization at $125^{\circ} \mathrm{C}$ and 1 bar.

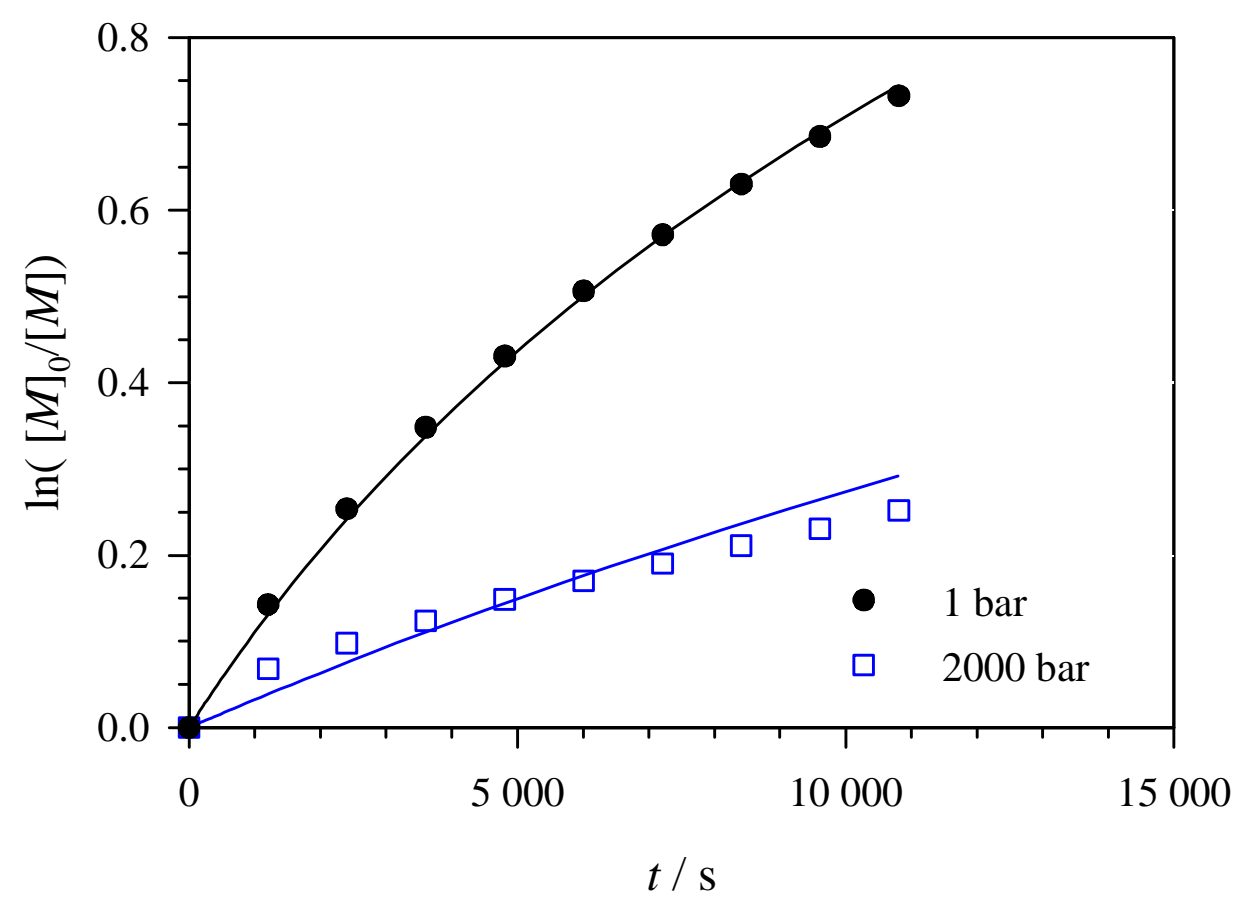

Fig. 4.7. Comparison of conversion index vs time plots for the spontaneous polymerization of styrene at $125^{\circ} \mathrm{C}$, at 1 bar and 2000 bar. The full lines present the simulated predictions. 
The calculated and measured molar mass distributions for 2000 bar are also very close to each other. These observations demonstrate the validity of the model which seems to be well suited for simulation of high pressure styrene FRP.

The next step toward parameter estimation is to carry out some simulations of controlled styrene polymerization for high-pressure conditions. This data will then be compared to experiments.

\subsubsection{Simulation of controlled styrene polymerization at high pressure}

The simulation of controlled styrene polymerization has been carried out in two steps. First, the pressure dependence of $k_{\mathrm{p}}$ and $k_{\mathrm{t}}$ has been taken into account before including estimated activation volumes for $k_{\mathrm{c}}$ and $k_{\mathrm{d}}$ relative to combination and dissociation of the dormant species. Results of simulations for high pressure controlled styrene polymerization are shown in Fig. 4.8. As with thermal spontaneous styrene polymerization, the kinetic of controlled styrene polymerization is strongly influenced by high pressure. The increase in $k_{\mathrm{p}}$ and the decrease in $k_{\mathrm{t}}$ with pressure lead to an important increase in the conversion index $\ln \left([M]_{0} /[M]\right)$ vs time plotted in Fig. 4.8.

Increasing pressure from 1 to 2000 bar enhances the conversion after 5 hours reaction time from 38 to $70 \%$. This effect results from an increase in $k_{\mathrm{p}}$ and a decrease in $k_{\mathrm{t}}$ with pressure. This increase in rate is associated with an unfavorable effect on the control of molecular weight in this initial polymerization period (Fig. 4.9). In fact, at low styrene conversion, the faster polymerization induces an increase in $M_{\mathrm{N}}$ from $7000 \mathrm{~g} \cdot \mathrm{mol}^{-1}$ at 1 bar to $14000 \mathrm{~g} \cdot \mathrm{mol}^{-1}$ at 2000 bar at $5 \%$ conversion (where control is not so effective), $M_{\mathrm{W}}$ and polydispersity. The deviation from ideal controlled polymerization observed at low conversion and high pressure is primarily due to propagation rate becoming fairly rapid at high pressure as compared to the reversible deactivation steps of the dormant species. At higher conversion no influence of pressure on $M_{\mathrm{N}}$ may be detected as is seen in Fig. 4.9. 


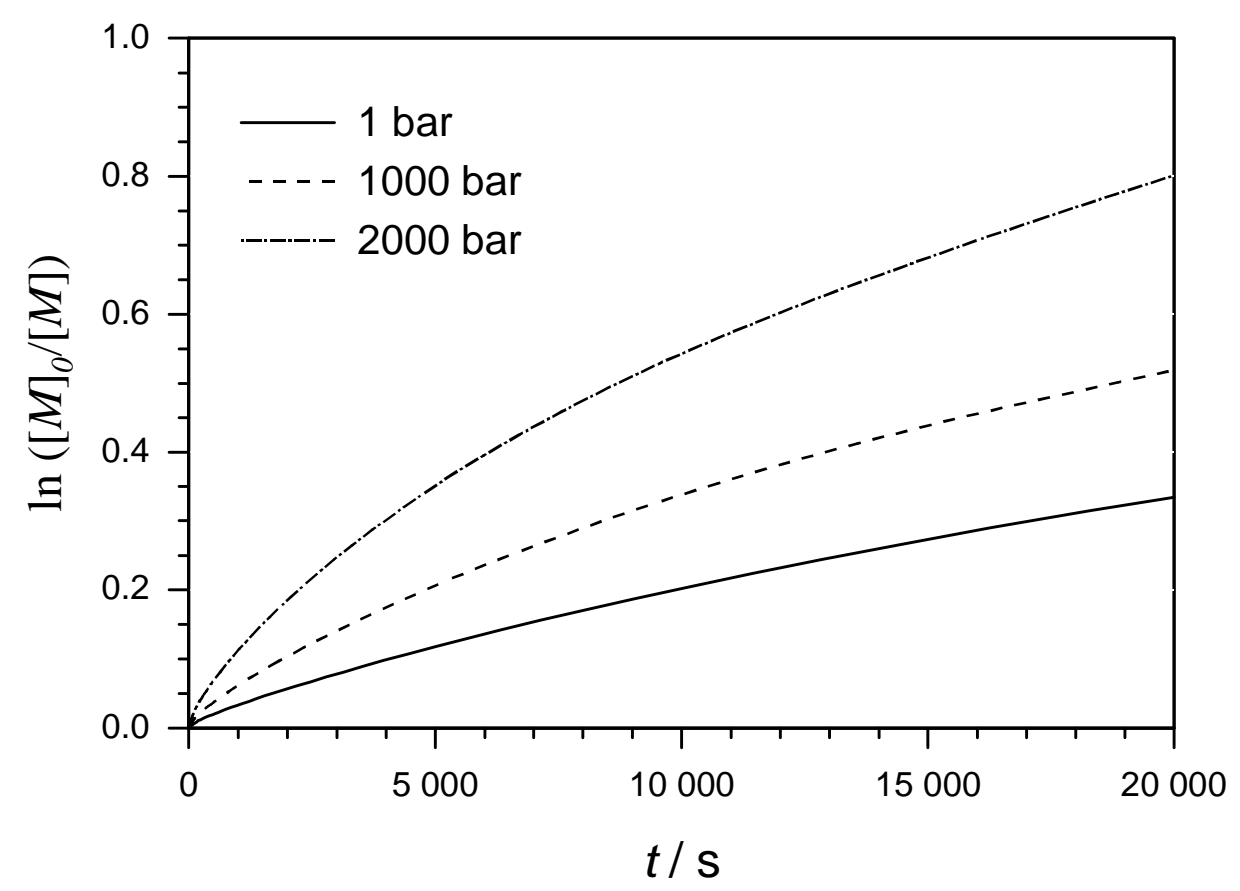

Fig. 4.8. $\quad$ Pressure dependence of simulated $\ln ([M] d /[M])$ vs time curves for styrene bulk polymerizations in the presence of $0.1 \mathrm{~mol} \%$ styryl-TEMPO at $125^{\circ} \mathrm{C}$ and at pressures up to 2000 bar.

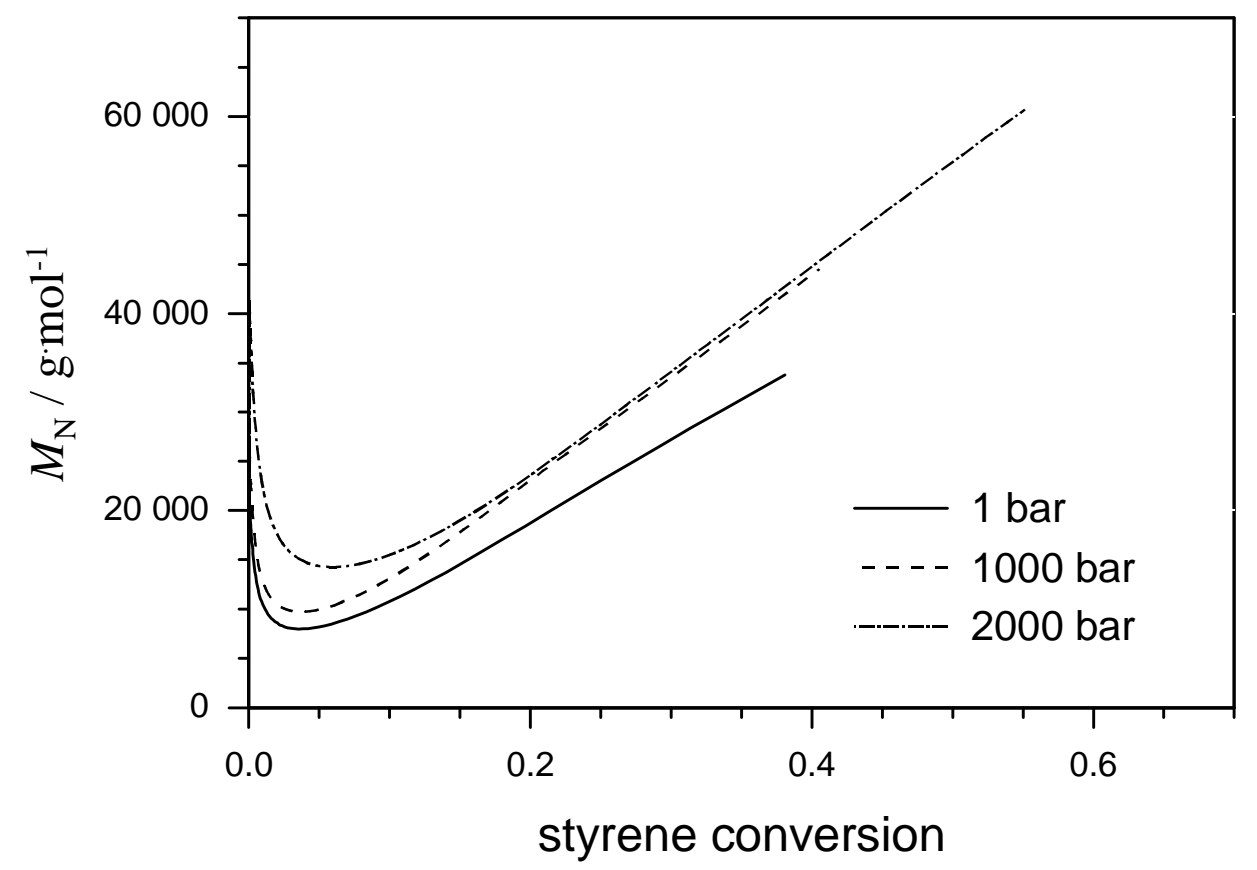

Fig. 4.9. Conversion dependence of molar mass $M_{N}$ for styrene polymerizations in the presence of 0.1 mol\% styryl-TEMPO at $125^{\circ} \mathrm{C}$, and pressures up to 2000 bar. All data are from simulations. 
Within the next step, simulations of controlled styrene polymerization are extended to include the pressure dependence of $k_{\mathrm{c}}$ and $k_{\mathrm{d}}$. The activation volume of $k_{\mathrm{c}}$ is assumed to be equal to $\Delta V^{\neq}\left(k_{\mathrm{c}}\right)=22 \mathrm{~cm}^{3} \cdot \mathrm{mol}^{-1}$, which is the activation volume of the termination rate coefficient. For the dissociation of the alkoxyamine it is assumed that the activation volume is close to $12 \mathrm{~cm}^{3} \cdot \mathrm{mol}^{-1}$, which is a typical value for a single-bond scission reaction. Further below, the activation volume $\Delta V^{\ddagger}\left(k_{\mathrm{d}}\right)$ will be systematically varied and the resulting effect will be investigated. The results of simulations of $\ln \left([M]_{0} /[M]\right)$ vs time and of $M_{\mathrm{N}}$ vs conversion for pressures up to 2000 bar are plotted in Figs 4.10 and 4.11.

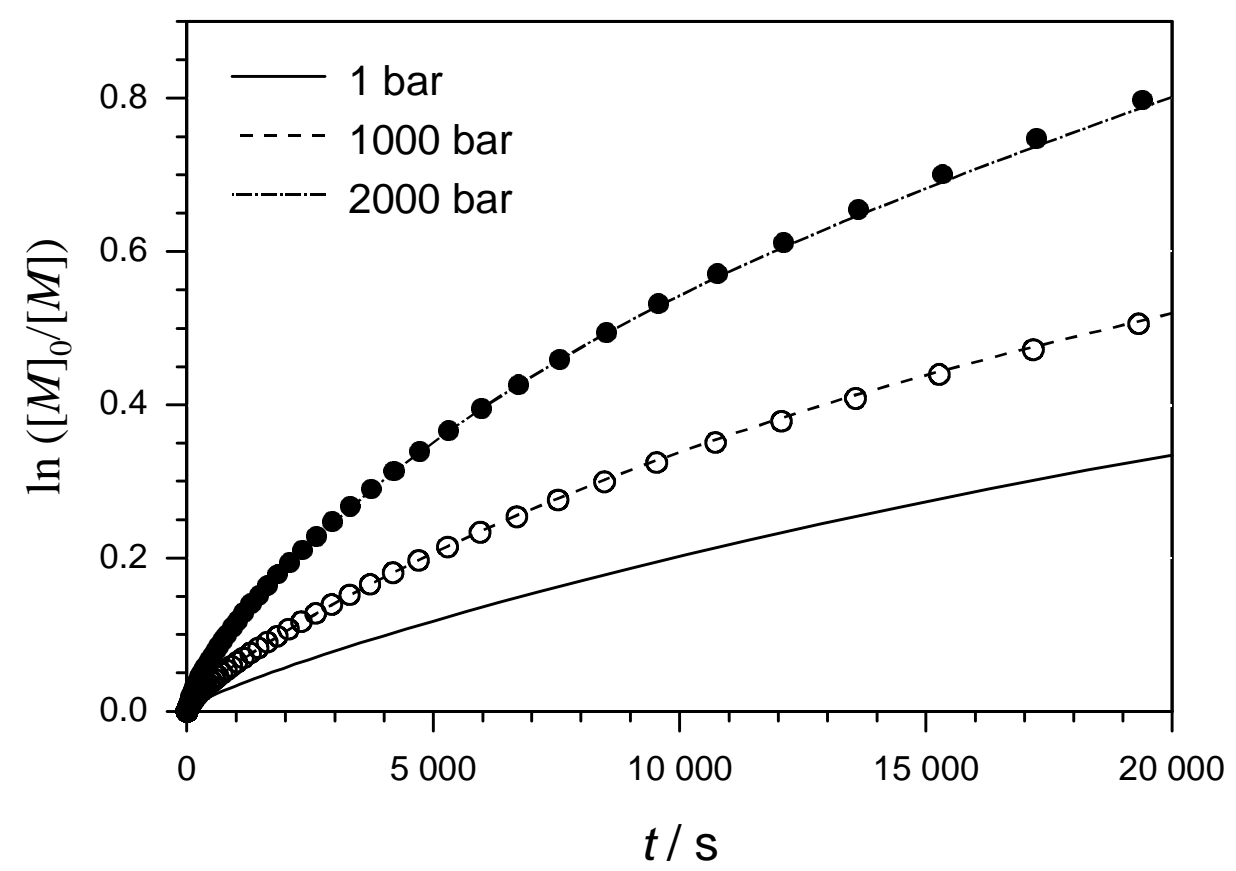

Fig. 4.10. $\quad$ Pressure dependence of simulated $\ln ([M] d /[M])$ vs time curves for styrene polymerizations in the presence of $0.1 \mathrm{~mol} \%$ styryl-TEMPO carried out at $125^{\circ} \mathrm{C}$, and pressures up to $2000 \mathrm{bar}\left(\Delta V^{\ddagger}\left(k_{c}\right)=22 \mathrm{~cm}^{3} \cdot \mathrm{mol}^{-1}, \Delta V^{\ddagger}\left(k_{d}\right)=12\right.$ $\left.\mathrm{cm}^{3} \cdot \mathrm{mol}^{-1}\right)$. The lines represent simulations without introducing pressure dependent $k_{c}$ and $k_{d}\left(\Delta V^{\neq}\left(k_{c}\right)\right.$ and $\left.\Delta V^{\neq}\left(k_{d}\right)=0\right)$. 


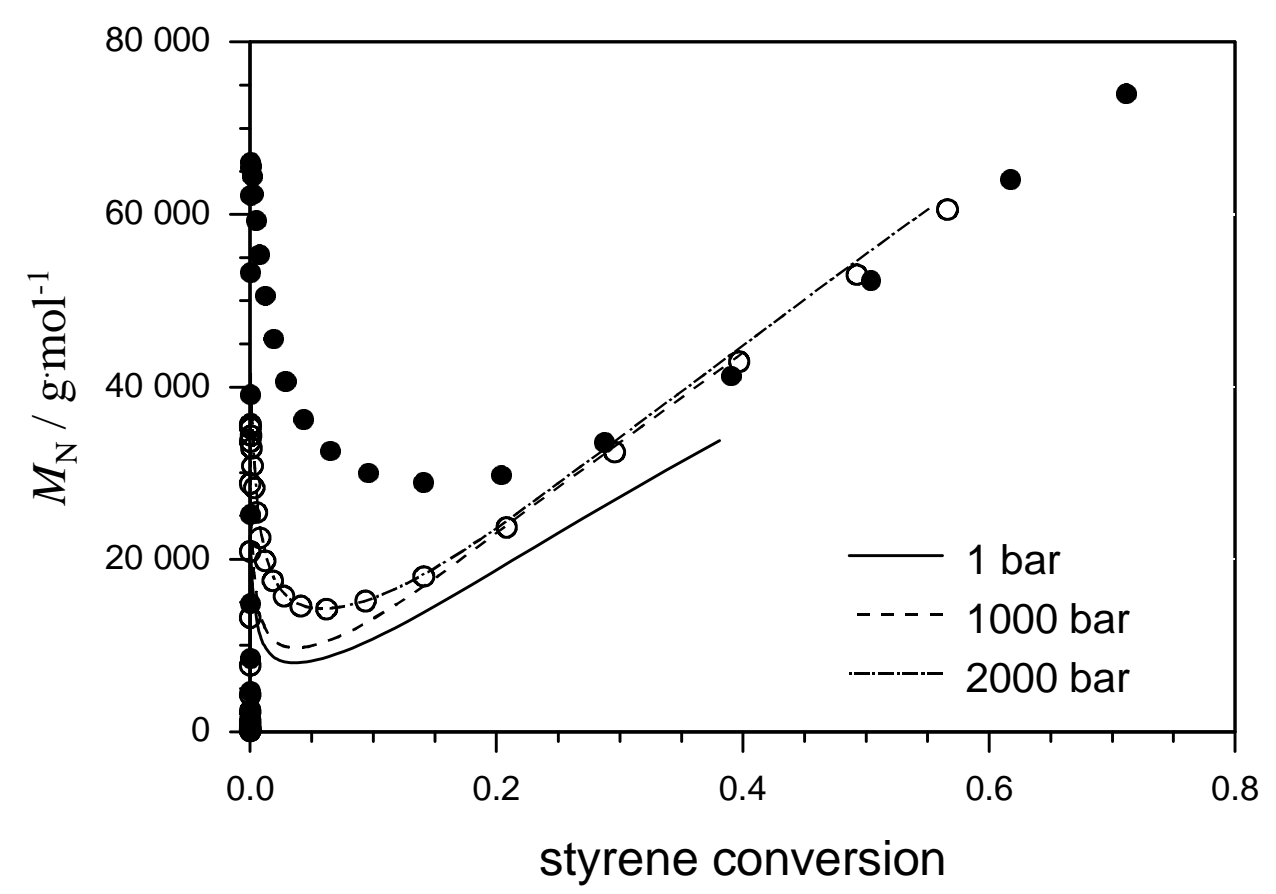

Fig. 4.11. $\quad$ Pressure dependence of simulated molar mass $M_{N}$ vs conversion for styrene polymerizations in the presence of 0.1 mol\% styryl-TEMPO carried out at $125^{\circ} \mathrm{C}$ and different pressures ( (-) 1 bar, (o) 1000 bar and (•) 2000 bar ). $\Delta V^{\ddagger}\left(k_{c}\right)=22 \mathrm{~cm}^{3} \cdot \mathrm{mol}^{-1}, \Delta V^{\neq}\left(k_{d}\right)=12 \mathrm{~cm}^{3} \cdot \mathrm{mol}^{-1}$. The lines represent simulations without introducing pressure dependent $k_{c}$ and $k_{d}\left(\Delta V^{\neq}\left(k_{c}\right)\right.$ and $\left.\Delta V^{\neq}\left(k_{d}\right)=0\right)$

Even at very high pressure (2000 bar), including the pressure dependence of $k_{\mathrm{c}}$ and $k_{\mathrm{d}}$ does not affect the rate of controlled polymerization. There is only a small effect because of the low activation volume difference between the two opposite reactions. The kinetic effect primarily results from the influence of pressure on $k_{\mathrm{p}}$ and $k_{\mathrm{t}}$.

According to the evolution of simulated molar masses plotted in Fig. 4.11, the activation volumes of $k_{\mathrm{c}}$ and $k_{\mathrm{d}}$ have a significant effect on $M_{\mathrm{N}}$. The lower pressure influence on $k_{\mathrm{d}}$ compared to the one on $k_{\mathrm{c}}$ (lower activation volume) induces an increase in the polymerization rate but leads to a poorer control of the polymerization, especially at low conversions. This phenomenon also happens at 1000 bar and is more pronounced at 2000 bar so that the extrapolation of the molar mass to zero conversion reaches about $10000 \mathrm{~g} \cdot \mathrm{mol}^{-1}$. At higher conversion (> $30 \%$ ), the control of styrene polymerization seems to be not affected. 
Summarized in Tab. 4.12 are the consequences of a variation of $\Delta V^{\ddagger}\left(k_{\mathrm{d}}\right)$, between 0 and $12 \mathrm{~cm}^{3} \cdot \mathrm{mol}^{-1}$ on the rate of controlled styrene polymerization at 2000 bar. Decreasing $\Delta V^{f}$ $\left(k_{\mathrm{d}}\right)$ allows for a faster decomposition of the dormant species and results in a faster rate of controlled polymerization.

\begin{tabular}{lcccc}
\hline time / s & $\Delta V^{\neq}\left(k_{\mathrm{d}}\right)=0$ & $\Delta V^{\neq}\left(k_{\mathrm{d}}\right)=4.25$ & $\Delta V^{\neq}\left(k_{\mathrm{d}}\right)=8.5$ & $\Delta V^{\neq}\left(k_{\mathrm{d}}\right)=12.2$ \\
& $\mathrm{~cm}^{3} \cdot \mathrm{mol}^{-1}$. & $\mathrm{cm}^{3} \cdot \mathrm{mol}^{-1}$ & $\mathrm{~cm}^{3} \cdot \mathrm{mol}^{-1}$ & $\mathrm{~cm}^{3} \cdot \mathrm{mol}^{-1}$ \\
3600 & 41.5 & 39.7 & 38.0 & 36.7 \\
7200 & 57.5 & 55.5 & 53.6 & 52.2 \\
10800 & 67.0 & 64.4 & 62.5 & 60.5 \\
14400 & 74.4 & 72.0 & 70.2 & 68.5 \\
18000 & 78.1 & 76.4 & 74.8 & 73.4 \\
\hline
\end{tabular}

Tab. 4.12. Simulated conversion for styrene polymerizations in the presence of $0.1 \mathrm{~mol} \%$ styryl-TEMPO carried out at $125^{\circ} \mathrm{C}$ and $2000 \mathrm{bar} . \Delta V^{\neq}\left(k_{c}\right)=22 \mathrm{~cm}^{3} \cdot \mathrm{mol}^{-1}$, $\Delta V^{\neq}\left(k_{d}\right)=0$ up to $12.2 \mathrm{~cm}^{3} \cdot \mathrm{mol}^{-1}$.

Increasing the activation volume $\Delta V^{\ddagger}\left(k_{\mathrm{d}}\right)$ from 0 to $12.2 \mathrm{~cm}^{3} \cdot \mathrm{mol}^{-1}$ lowers the polymerization rate by about $5 \%$ over a 3 hours reaction period. This effect is due to the slower dissociation of the dormant species. $\Delta V^{\neq}\left(k_{\mathrm{d}}\right)$ has a relative small influence on polymerization rate as compared to the influence of $\Delta V^{\ddagger}\left(k_{\mathrm{p}}\right)$ on polymerization rate. The $M_{\mathrm{N}}$ evolution plotted in Fig. 4.13 for different activation volumes of $k_{\mathrm{d}}$ shows a better control at low conversion if $\Delta V^{\neq}\left(k_{\mathrm{d}}\right)$ is small. The large deviation from ideal controlled polymerization at low conversion is essentially due to the rapid propagation rate at high pressure compared to the reversible deactivation process of the dormant species. 


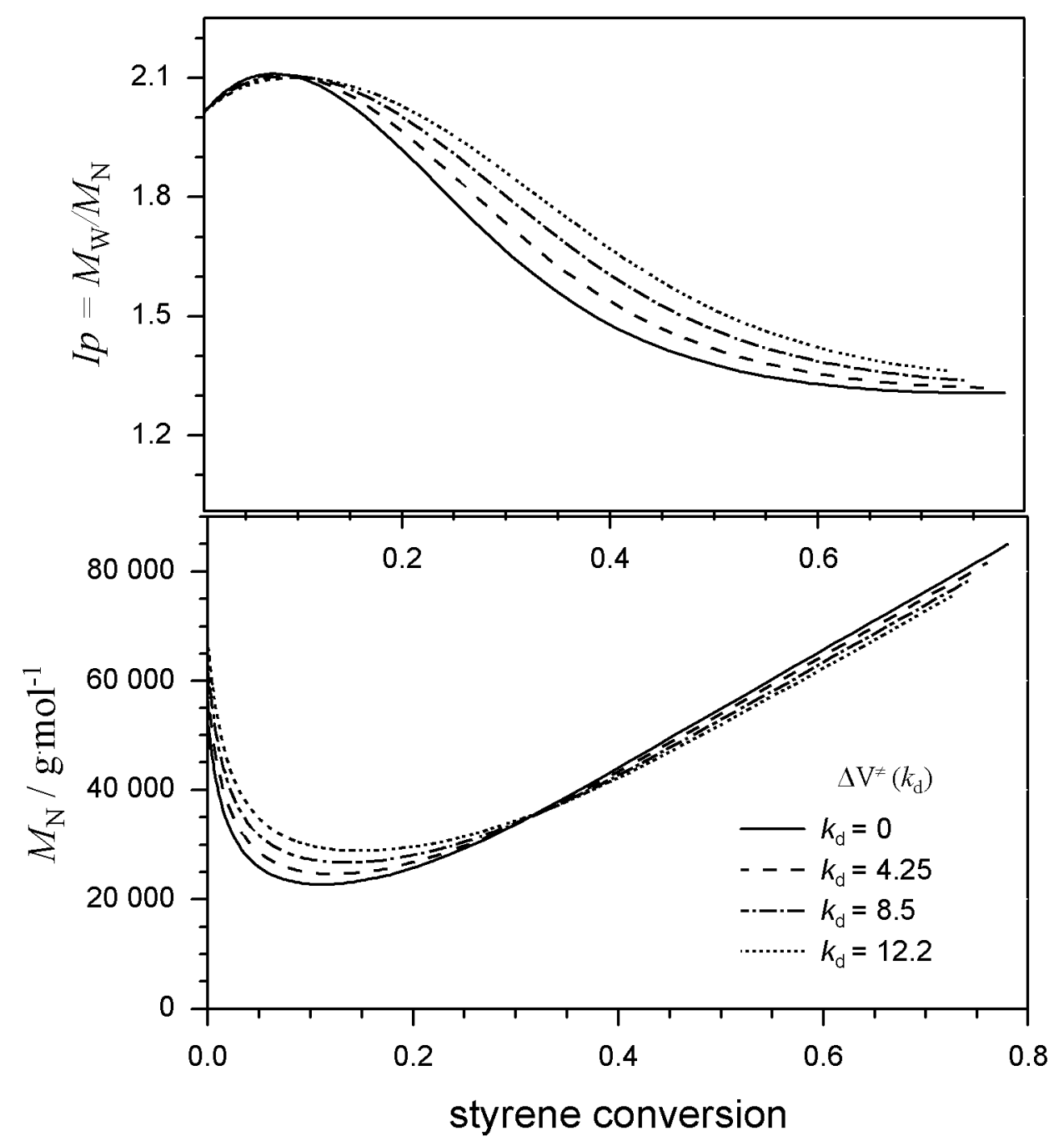

Fig. 4.13. Conversion dependence of simulated number-average molar mass, $M_{N}$, and polydispersity index, Ip, for styrene polymerizations in the presence of 0.1 mol\% styryl-TEMPO carried out at $125^{\circ} \mathrm{C}$ and 2000 bar. Different activation volumes $\Delta V^{\neq}\left(k_{d}\right)$ from 0 to $12.2 \mathrm{~cm}^{3} \cdot \mathrm{mol}^{-1}$ are used in the calculations. $\Delta V^{\neq}\left(k_{c}\right)=22 \mathrm{~cm}^{3} \cdot \mathrm{mol}^{-1}$ is kept constant.

\subsection{Kinetic model for nitroxide-mediated ethene homopolymerization}

\subsubsection{Elementary reactions in free-radical ethene homopolymerization}

Computer models for ethene high-pressure radical polymerization have been published by several authors ${ }^{16-18}$. In their articles, the theoretical property and conceptual study of the sensitivity of some operation factors and kinetic coefficients upon conversion or 
molecular structure have been discussed. Agreement of the model with actual operation in a commercial plant was obtained by Goto ${ }^{19}$.

High-pressure polymerization of ethene is performed under very severe conditions such as pressures from 1000 to 3000 bar and temperatures from 150 to $350^{\circ} \mathrm{C}$. For development of our model and for a good comprehension of the observed calculated results, it is important to use exact reaction rates obtained in similar conditions used in this work. Wellaccepted kinetic schemes are found in the literature for propagation and termination as well as for transfer reactions to monomer. Rate coefficients have been determined by Schweer ${ }^{20}$ (for propagation and termination, including their conversion dependence) and by Buback et al. ${ }^{21}$ (for chain-transfer to monomer). The formation of short chain branching is modeled as described by Goto ${ }^{19}$ using a single reaction step for the back-biting reaction. The kinetic schemes describing transfer reaction to polymer are still under discussion. Here it is treated as a reaction sequence producing a secondary radical capable of $\beta$-scission or propagation ${ }^{22}$.

$$
\begin{array}{ll}
I \stackrel{f k_{\mathrm{I}} \longrightarrow}{\longrightarrow} R_{1} & \text { initiation } \\
R_{m}+M \stackrel{k_{\mathrm{p}}}{\longrightarrow} R_{m+1} & \text { propagation } \\
R_{m}+R_{n} \stackrel{k_{\mathrm{td}}}{\longrightarrow} P_{m}+P_{n} & \text { termination } \\
R_{m}+R_{n} \stackrel{k_{\mathrm{tc}}}{\longrightarrow} P_{m+n} & \text { chain transfer to monomer } \\
R_{n}+M \stackrel{k_{\mathrm{tr}, \mathrm{M}}}{\longrightarrow} P_{n}+R_{1} & \text { chain transfer to solvent } \\
R_{n}+S \stackrel{k_{\mathrm{tr}, \mathrm{S}}}{\longrightarrow} P_{n}+R_{1} & \text { back-biting (intramolecular transfer to polymer) } \\
R_{n} \stackrel{k_{\mathrm{SCB}}}{\longrightarrow} R_{n}+S C B & \text { chain transfer to polymer (intermolecular transfer) } \\
R_{m}+P_{n} \stackrel{k_{\mathrm{tr}, \mathrm{P}}}{\longrightarrow} P_{m}+R_{n, \mathrm{sec}} & \\
R_{n, \mathrm{sec}}+E \stackrel{k_{\mathrm{p}}}{\longrightarrow} R_{\mathrm{n}+1}+L C B & \text { propagation of secondary macroradical } \\
R_{n, \mathrm{sec}} \stackrel{k_{\mathrm{B}}}{\longrightarrow} P_{n-k}+R_{k} & \text { B-scission of secondary radical }
\end{array}
$$

Fig. 4.14. Elementary reaction steps implemented for modeling ethene homopolymerization 
where $I$ is the initiator, type peroxide; $R_{n}$ is the radical of chain length $n ; M$ is the monomer (ethene); $\mathrm{S}$ is the solvent ( $n$-heptane or toluene); $\mathrm{P}_{\mathrm{n}}$ is the dead polymer of chain length $\mathrm{n}$; $\mathrm{SCB}$ is short chain branch; LCB is long chain branch; $k_{\mathrm{p}}, k_{\mathrm{tc}}, k_{\mathrm{tr}, \mathrm{M}}$, etc. are the kinetic rate coefficients.

The elementary reactions and kinetic forms, reported in Fig. 4.14, were adopted for the model. All of the important elementary reactions which have been discussed by many workers in this field were incorporated ${ }^{23}$.

A rate constant may be written in the following form :

$$
k=k_{0} \exp \left(-E_{\mathrm{a}} \cdot(\mathrm{R} \cdot T)^{-1}-P \cdot \Delta V^{ \pm} \cdot(\mathrm{R} \cdot T)^{-1}\right)
$$

where $k_{0}$ is the pre-exponential factor; $E_{\mathrm{a}}$ is the activation energy; $\Delta V^{\neq}$is the activation volume; $T$ is the temperature; $P$ is the pressure and $\mathrm{R}$ is the gas constant.

Equations 4.1 to 4.3 represent the minimum set of reactions steps assuming ideal polymerization kinetics. Transfer reactions to monomer and solvent are taken into account in Equations 4.4 and 4.5. Most of these reaction steps have been used in literature on simulations, however transfer reactions to polymer and induced processes (Eq. 4.6 to 4.8) may have a significant influence on molar mass distributions.

Eq. 4.6 represents the intramolecular transfer to polymer (backbiting) preferably forming a six-membered transition state at the chain end forming a short chain branch $(\mathrm{SCB})^{24}$ as shown in Table 4.14. The short chain branch formation has been intensively studied and many authors ${ }^{25-29}$ concluded that most of the short chains produced in LDPE are $n$-butyl and ethyl groups. Only a small amount of $n$-amyl and $n$-hexyl groups have been observed. The ethyl group formation results of two consecutive backbiting reactions. It is well known that this short chain branching influences the morphology and the properties of semicristallized polyethylene. LDPE may contain between 10 and up to 40 SCB per $1000 \mathrm{C}$-atom. The influence of synthesis conditions on short chain branching has been examined by Luft et al. ${ }^{30}$. The authors showed that the short chain branching concentration increases toward higher temperature and lower pressure. 
Eq 4.7 shows the intermolecular transfer to polymer by abstracting a hydrogen atom from repeat units in the center of the backbone. The resulting secondary macroradical may either propagate to a long chain branch ( $\mathrm{LCB}$, Eq. 4.8) or undergo a $\beta$-scission reaction (Eq. 4.9) ${ }^{7}$.

Long chain branching in LDPE ${ }^{31}$ is responsible for the high polydispersities and for the rheologic properties (such as viscosity and viscoelastic properties). The number of LCB per 1000 C-atom may be determined by gel permeation chromatography, viscosity measurement and ${ }^{13} \mathrm{C}$-nuclear magnetic resonance. Luft et al. ${ }^{30}$ have studied the influence of synthesis conditions on LCB, the concentration of that decreases with temperature and pressure.

Alternatively to the propagation, a primary, secondary or tertiary radical may undergo a $\beta$-scission reaction producing a vinyl-end group and a new primary radical that starts macromolecular growth. Vinyl groups from B-scission of a primary or secondary radical may be produced through termination by disproportionation or by transfer to polymer. Vinylidene groups $\left(>\mathrm{CH}=\mathrm{CH}_{2}\right)$ are produced by scission of tertiary radicals. Experimental observations showed the presence of trans-vinyl groups $(-\mathrm{CH}=\mathrm{CH}-)$ for which the formation mechanism is not clear ${ }^{34}$. They occur in very low concentration.

Nevertheless, $\beta$-scission of macroradicals with the radical function at the chain end seems somehow unrealistic. For all these reasons, transfer to polymer and $\beta$-scission reactions should be modeled as previously suggested.

It is well known that ethene may undergo an exothermic auto-decomposition. The activation energy is about $125 \mathrm{~kJ} \cdot \mathrm{mol}^{-1} 33$. Upon ethene decomposition, propagation becomes extremely fast, producing a drastic temperature and pressure increase. It is assumed that spontaneous ethene decomposition is due to local hot spots.

The reactor is considered as an ideal stirred batch reactor. Ideal micromixture are presumed and temperature and pressure profiles, by the beginning of the reaction are taken into account. 


\subsubsection{Rate constants in free-radical ethene homopolymerization}

The situation with respect to the availability of rate coefficients is very fortunate for ethene homopolymerization. Propagation $\left(k_{\mathrm{p}}\right)$ and termination $\left(k_{\mathrm{t}}\right)$ rate coefficients in combination with their dependence on conversion $(X)$ have been determined by laser single pulse experiments (Eq. 4.11-4.15) ${ }^{20}$.

$k_{\mathrm{p}}^{0} / \mathrm{L} \cdot \mathrm{mol}^{-1} \cdot \mathrm{s}^{-1}=1.88 \cdot 10^{7} \cdot \exp -\left(4125 \cdot(T / \mathrm{K})^{-1}-0.325 \cdot(P /\right.$ bar $\left.) \cdot(T / \mathrm{K})^{-1}\right)$

The subsequent values for $k_{\mathrm{t}}$ refer to the IUPAC definition :

$$
\begin{aligned}
& \partial c_{R} / \partial t=-2 k_{\mathrm{t}} \cdot c_{R}^{2} \\
& k_{\mathrm{t}}^{0} / \mathrm{L} \cdot \mathrm{mol}^{-1} \cdot \mathrm{s}^{-1}=8.111 \cdot 10^{7} \cdot \exp -\left(553 \cdot(T / \mathrm{K})^{-1}+0.190 \cdot(P / \text { bar }) \cdot(T / \mathrm{K})^{-1}\right) \\
& k_{\mathrm{p}}(X, \eta, T, P)=\frac{k_{\mathrm{p}}^{0}}{1+\frac{k_{\mathrm{p}}^{0}}{1.13 \cdot 10^{10}} \cdot \eta_{r}} \\
& k_{\mathrm{t}}(X, \eta, T, P)=\left(0.832 \cdot \frac{1}{\eta_{r}}+8.04 \cdot 10^{-6} \cdot(1-X) \cdot k_{\mathrm{p}}(X, \eta, T, P)\right) \cdot k_{\mathrm{t}}^{0} \\
& \eta_{r}(X)=10^{(5.39 \cdot X+3.7 \cdot \sqrt{X)}}
\end{aligned}
$$

where $X$ represents the ethene conversion in a range from 0 to 1 . It is assumed that the ratio of termination by combination to termination by disproportionation $k_{\mathrm{tc}} / k_{\mathrm{td}}$ is independent of monomer conversion. The determination of this ratio by Lee and Marano ${ }^{18}$ as a function of temperature and pressure (Eq. 4.16) and a combination with Eq. 4.14 yields an expression of these rate coefficients as a function of temperature, pressure and conversion.

$$
\frac{k_{\mathrm{tc}}}{k_{\mathrm{td}}}=3.321 \cdot \exp -\left(150 \cdot(T / \mathrm{K})^{-1}-0.108 \cdot(P / \mathrm{bar}) \cdot(T / \mathrm{K})^{-1}\right)
$$

The rate coefficient for transfer to monomer was determined by Buback et al. ${ }^{8,21}$, by studying low conversion ethene homopolymerizations. The investigations in the field of 
number average $M_{\mathrm{N}}$ of the molar mass distributions permitted the description of the temperature and pressure dependence expressed by Eq. 4.17.

$$
k_{\mathrm{tr}, \mathrm{M}} / L \cdot \mathrm{mol}^{-1} \cdot \mathrm{s}^{-1}=3.42 \cdot 10^{8} \cdot \exp -\left(9135 \cdot(T / K)^{-1}-0.067 \cdot(P / \mathrm{bar}) \cdot(T / K)^{-1}\right)
$$

The value of chain transfer constant to solvent $C$ s defined as the $k_{\mathrm{tr}, \mathrm{S}} / k_{\mathrm{p}}$ may be obtained from the kinetic formation of the degree of polymerization as described by van der Molen ${ }^{20}$. After combination with $k_{\mathrm{p}}$, the rate coefficient for the transfer to solvent $k_{\mathrm{tr}, \mathrm{S}}$ may be calculated.

Rate coefficients for backbiting, transfer to polymer and $\beta$-scission are not so easily accessible. Goto et al. ${ }^{19}$ reported detailed experimental data in extended range of temperature and pressure. They used a different description of transfer reactions to polymer and following processes. These data have been fitted to the kinetic model by Busch ${ }^{36}$ and rate coefficients for backbiting have been deduced.

$$
k_{\mathrm{SCB}} / s^{-1}=1.95 \cdot 10^{8} \cdot \exp -\left(5383 \cdot(T / \mathrm{K})^{-1}-0.280 \cdot(P / \text { bar }) \cdot(T / \mathrm{K})^{-1}\right)
$$

This is derived from the ratio of $k_{\mathrm{SCB}}$ over $k_{\mathrm{p}}$ correlated to the experimental data and has been calculated using $k_{\mathrm{p}}$ as described in Eq. 4.11.

Busch ${ }^{36}$ also deduced rate coefficients for transfer to polymer and by calculating the overall probability of $\beta$-scission (by summing up those of the formation of vinyl and vinylidene) finally provides the rate coefficient for the $\beta$-scission reactions. The frequency factor of the Arrhenius equation for $k_{\beta}$ has to be adapted by using few experimental data for fitting. Indeed, technical ethene polymerization may be well described by applying the model developed up to this point ${ }^{37}$. 


\subsubsection{Introduction of reversible nitroxide radical trapping reactions into the model for ethene free radical polymerization}

The model considered up to now does not include reversible spin trap reaction (Eq. 4.19) to control free radical polymerization. Very simple reactions have been added in order to describe the nitroxide mediated ethene polymerization under high pressure.

$R_{n} N \stackrel{k_{\mathrm{c}} / k_{\mathrm{d}}}{\longrightarrow} R_{n}+N \quad$ reversible deactivation of the growing radical

where $\mathrm{N}$ is the nitroxide or persistent radical, and $\mathrm{R}_{\mathrm{n}} \mathrm{N}$ is the nitroxide trapped radical of chain length $\mathrm{n}$ or dormant species. $k_{\mathrm{c}}$ and $k_{\mathrm{d}}$ are the rate coefficient of combination and dissociation, respectively.

The same hypothesis has been made for styrene controlled polymerization concerning the deactivation of the growing radical because no data are available in literature. It is assumed that the combination of two different types of radicals (N-oxyl - alkyl radicals) and termination reaction (recombination of two alkyl radicals), that pressure dependence is very low, should be similar so that $k_{\mathrm{c} \text { hexyl-TEMPO }} \approx k_{\mathrm{t}}$, as described in Eq. 4.12 and 4.14.

The kinetic rate coefficient of the dissociation of the dormant species has been assumed to be not chain length dependent.

The temperature dependence of the dissociation of the alkoxyamine methyl-TEMPO, given by Fischer ${ }^{37}$, is presented in Eq. 4.20. The very high activation energy (180-190 $\mathrm{kJ} \cdot \mathrm{mol}^{-1}$ ) of the decomposition of alkyl-TEMPO bond shows the high stability of the alkoxyamine. It suggests that this bond dissociation will be low, and the control of molar mass evolution of TEMPO-mediated ethene polymerization should be poor at low conversion.

$$
k_{\mathrm{d}} / s^{-1}=2.5 \cdot 10^{15} \cdot \exp -\left(22250 \cdot(T / \mathrm{K})^{-1}\right)
$$




\subsubsection{Simulation of ethene polymerization in the presence of a TEMPO-based} alkoxyamine

Hexyl-TEMPO has been chosen as mediating species of ethene polymerization for several reasons. First of all, an initiator may present efficiency problems and a nitroxide may thermally decompose. On the other hand, the polyethylene-TEMPO structure is close to the hexyl-TEMPO structure, the bond dissociation rate coefficient of which has been determined by Fischer ${ }^{37}$.

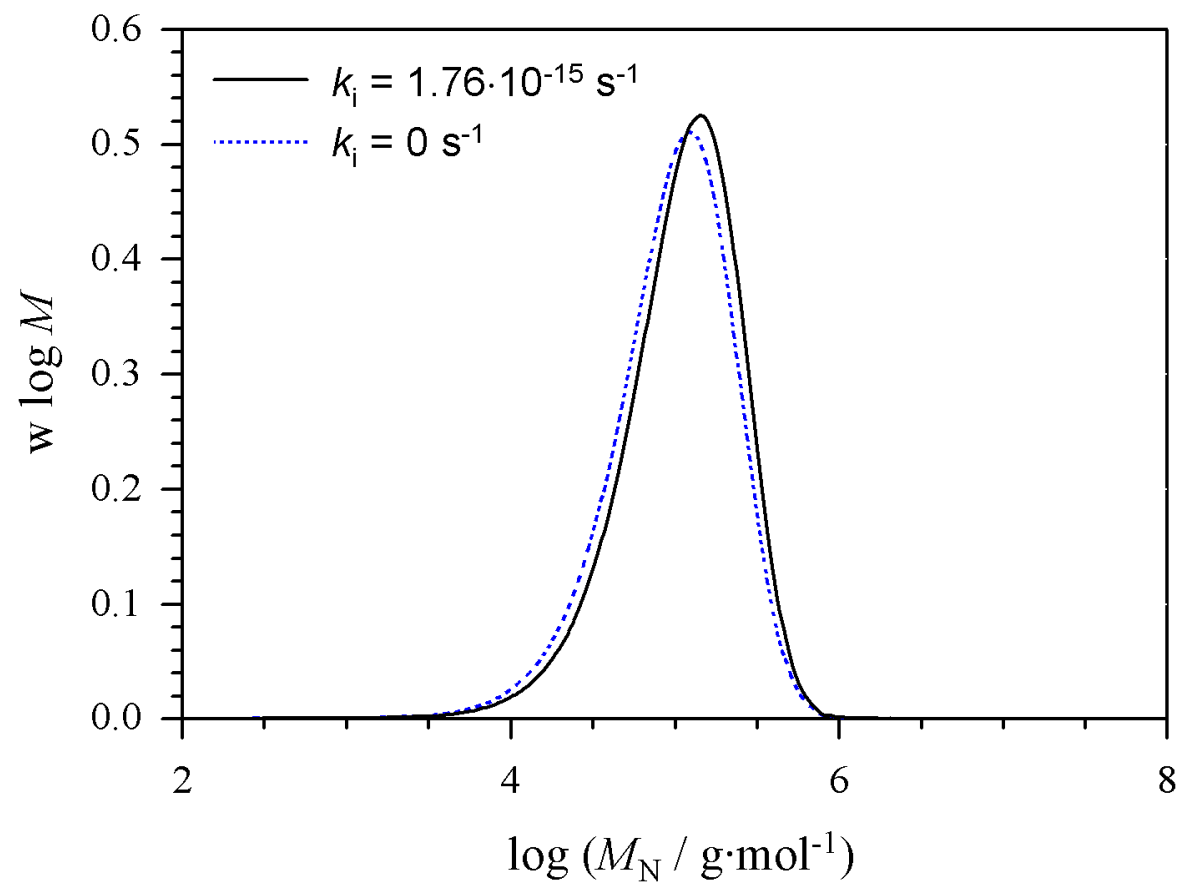

Fig. 4.15 Molecular weight distribution of dormant species simulated for ethene polymerizations carried out at $210^{\circ} \mathrm{C}$ and 2000 bar in the presence of $100 \mathrm{ppm}$ hexyl-TEMPO, with (solid line) and without (dotted line) spontaneous ethene polymerization. MWDs measured after $16 \%$ ethene conversion.

Simulations were carried out for $210^{\circ} \mathrm{C}$ and 2000 bar in order to derive the kinetics of spontaneous ethene polymerization by fitting the experimental data (about $11 \%$ conversion after 3 hours). $k_{\mathrm{i}}$ is found to be $1.76 \cdot 10^{-15} \mathrm{~s}^{-1}$. In the presence of $100 \mathrm{ppm}$ of hexyl-TEMPO, the conversion index $\ln \left([M]_{0} /[M]\right)$ increases linearly with time. $16 \%$ conversion are reached after 3 hours. The hexyl-TEMPO concentration during the polymerization has been calculated. It turns out that only $47.7 \%$ are consumed in the reaction time interval up to 3 
hours. The long-chain branching (LCB) concentration was found to be very low $(0.25$ $\mathrm{LCB} / 1000 \mathrm{C}$ ) compared to classic LDPE (10 to $30 \mathrm{LCB} / 1000 \mathrm{C}$ ). As depicted in Figure 4.15, the MWD of the dormant species is fairly broad and is not characteristic of a controlled polymerization. The calculated polydispersity is about 2.12 and the concentration of dormant species is very close to the concentration of dead polymer resulting from termination and transfer processes $\left(1.07 \cdot 10^{-3}\right.$ and $7.7 \cdot 10^{-4} \mathrm{~mol} \cdot \mathrm{l}^{-1}$, respectively). The constant molar mass of the dormant species with conversion (Fig. 4.16) shows that this polymerization is not controlled by hexyl-TEMPO.

The simulation of hexyl-TEMPO-mediated ethene polymerization was carried out up to high conversion. The ethene polymerization is also not controlled at high monomer conversion. After $25 \%$ conversion, the concentration of dormant species becomes lower than the concentration of dead polymer and the molar mass evolution stays constant. Transfer and termination processes seem to dominate the MWD.

A second run of simulations of hexyl-TEMPO-mediated ethene polymerization was carried out in the absence of spontaneous ethene polymerization. It turns out that there is no difference for the cases with and without thermal initiation, as shown in Figs 4.15 and 4.16, for MWD and molecular weight evolution with conversion. Even ethene conversion, sleeper and dead polymer concentrations are only slightly influenced by spontaneous ethene polymerization.

Simulations were performed at different conditions (from 150 to $250^{\circ} \mathrm{C}$ and from 1500 to 2000 bar) with different alkoxyamine concentrations. In none of them indications of controlled ethene polymerization was found. It was decided to try to model ethene for the hypothetical situation that the mediator-specific rate coefficients may be taken such as in the TEMPO-mediated styrene polymerization . Starting from controlled styrene polymerization, the ethene rate coefficients (for $200^{\circ} \mathrm{C}$ and 2000 bar) will be included one after the other in order to see which effect is responsible for the loss of polymerization control. 


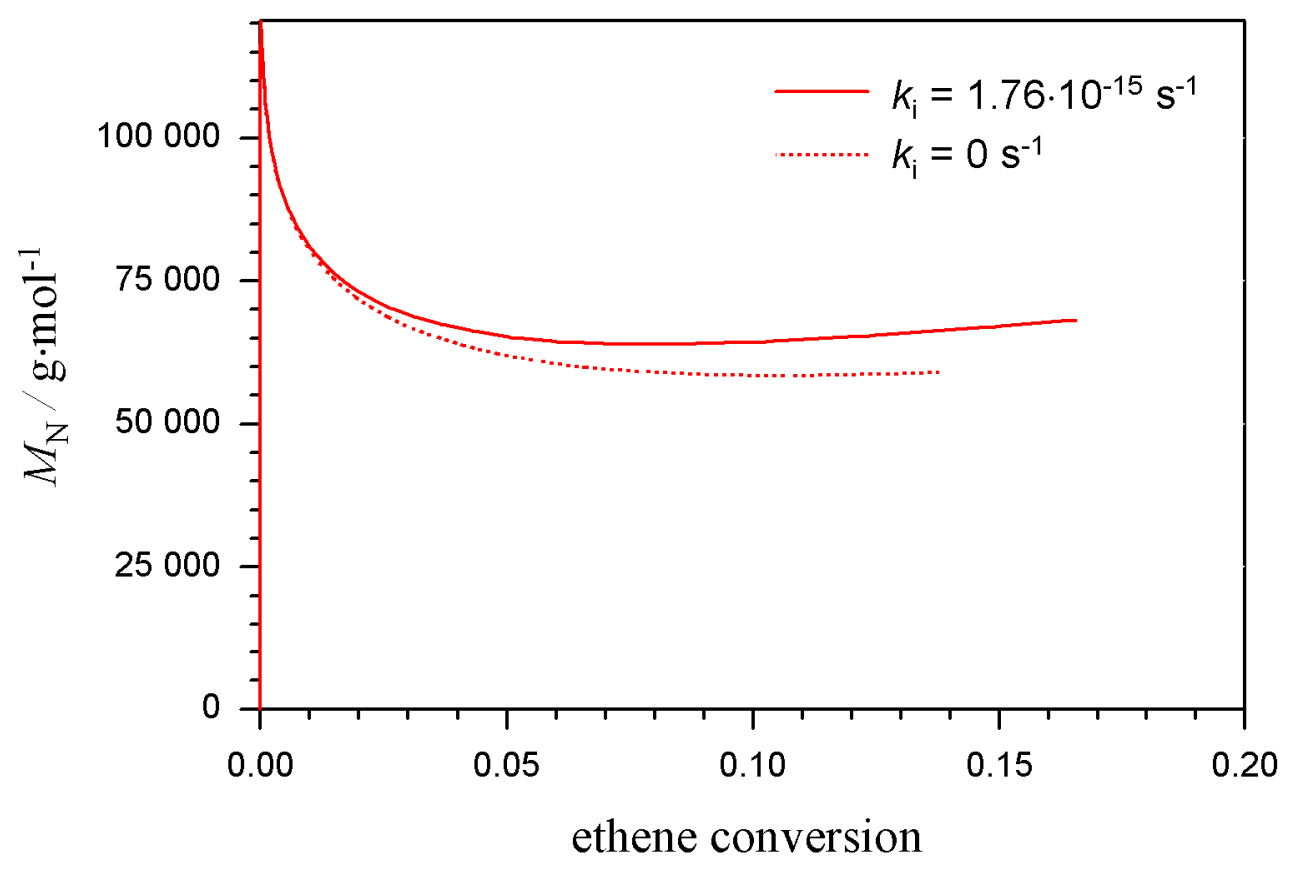

Fig. 4.16 Molecular weight of the dormant species as a function of ethene conversion simulated for ethene polymerization carried out at $210^{\circ} \mathrm{C}$ and 2000 bar in the presence of 100 ppm hexyl-TEMPO, with (solid line) and without (dotted line) of spontaneous ethene polymerization.

\subsubsection{Persistent radical effect as described by Fischer}

Before CRP was introduced, it was difficult to imagine that a free-radical polymerization may proceed without termination. Otsu et al. ${ }^{39}$ first observed that termination can be minimized when the growing polymer chain undergoes a reversible dissociation at the chain end into a transient propagating radical and a persistent radical. If the equilibrium constant of the reversible dissociation (Eq. 4.19) is sufficiently small, there will be only a low radical concentration and then a low termination rate. The concentration of transient radicals during styrene polymerization has been estimated as $10^{-7} \mathrm{M}$ or less. Direct ESR measurements showed quite high concentrations of the persistent species $\mathrm{N}$ of about $10^{-3} \mathrm{M}^{40-42}$. If termination is absent, the concentration of the transient and the persistent species should be equal, but this is clearly not the case. Fischer et al. ${ }^{43}$ concluded that termination can not be neglected and plays a crucial role for the control and the excess of the persistent over the transient species. Following Finke, the phenomenon is now properly termed the Persistent Radical Effect (see section 2.2). 
In reaction 4.19 , transient radicals and persistent radicals are formed at the same rate and in equal amount. The persistent radicals do not react with each other, but couple with transient radicals in a reversible process. On the other hand, the transient radicals do not only react with the persistent species but also undergo irreversible self-termination. Hence, by simple stoechiometry, self-termination induces an excess of persistent radicals. Therefore, the deactivation of the growing radical becomes more and more favored and dominant. The selftermination of growing radicals becomes increasingly less important, but never completely ceases ${ }^{45}$.

It must be noted that the equilibrium of the reversible dissociation exists only under certain conditions. The constant $K$ constant obeys

$$
K=k_{\mathrm{d}} / k_{\mathrm{c}}<[I]_{0} \cdot k_{\mathrm{c}} / 4 k_{\mathrm{t}}
$$

were $[\mathrm{I}]_{0}$ is the initial initiator concentration.

i.e. $K$ is lower than an upper limiting value. Decreasing the parameter $k_{\mathrm{c}}$, may have the consequence that the condition given in Eq. 4.21 is no longer fulfilled. The transient species then reaches an intermediate steady state ${ }^{45}$.

In order to understand why the TEMPO-mediated ethene polymerization does not present characteristics of controlled polymerization, a model for styrene polymerization was used which shows the persistent radical effect and ethene parameters were gradually introduced into the simulation. Polymerization conditions and rate coefficients are those used by Fischer ${ }^{34}$ at $125^{\circ} \mathrm{C}$ and 1 bar. The styrene model presented before (see section 4.1.1) was simplified: only self-initiation, propagation, termination and reversible deactivation of the transient were taken into account. Polymerization was initiated by a conventional initiator (BPO) in the presence of TEMPO $([\mathrm{BPO}]=0.04 \mathrm{M},[\mathrm{TEMPO}]=0.08 \mathrm{M})$. The evolution of the different species was followed during the simulated styrene polymerization and is depicted in Figure 4.17. 
At early polymerization times, the concentration of the transient radical stays very low since these radicals are trapped by TEMPO which leads to the formation of styryl-1-mers. As soon as BPO is consumed, the TEMPO concentration increases sharply and the concentration of growing free radical increases until a quasi-steady state is reached which exists for an extended period of the polymerization. The low polydispersity of the dormant species, the low dead polymer concentration and the growing evolution of the sleeper molar mass are typical for controlled polymerization. PREDICI ${ }^{\circledR}$ calculations are capable of describing this persistent radical effect in the same way as do the analytical expressions provided by Fischer ${ }^{34}$.

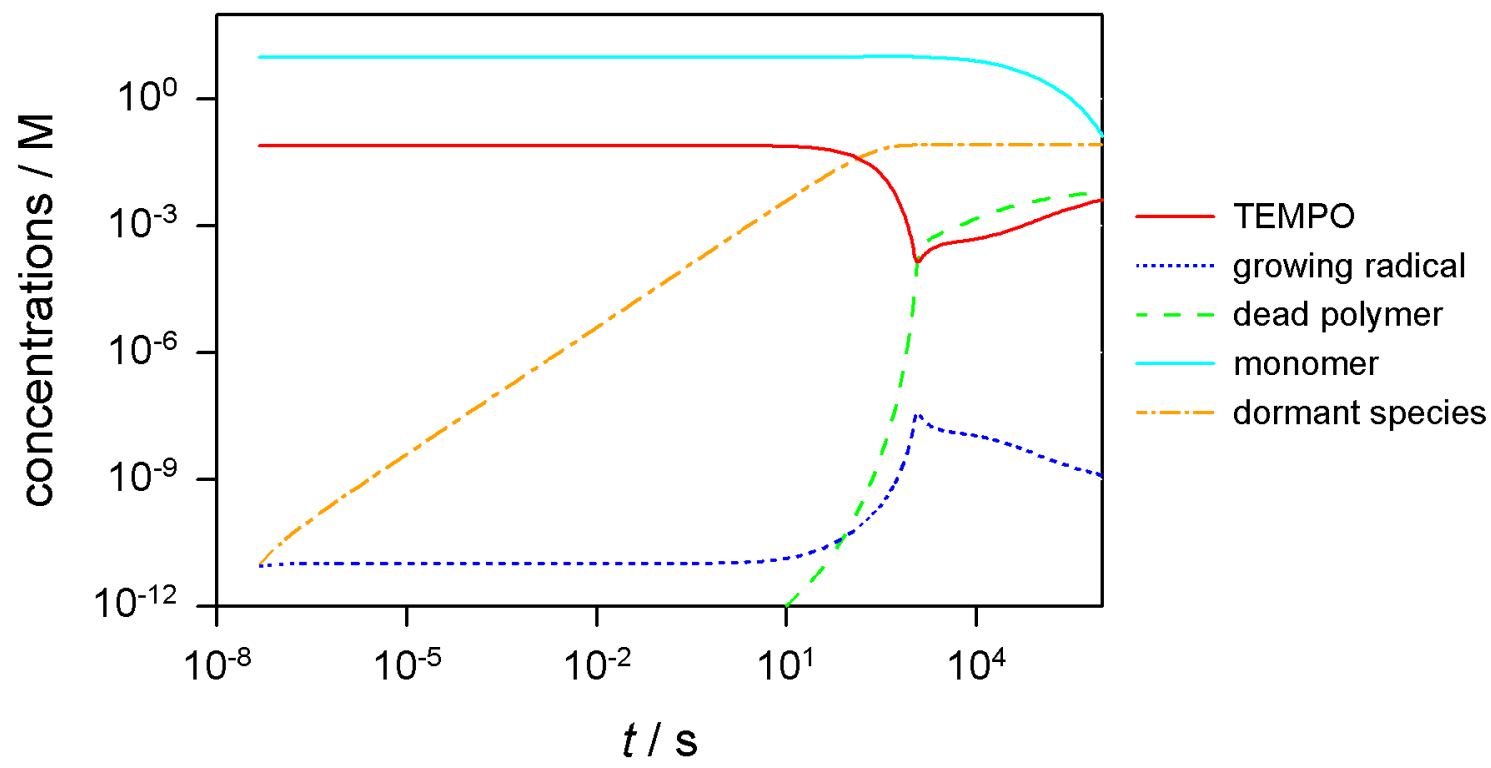

Fig. 4.17 Time evolution of concentrations of growing and persistent radicals, of dormant species and of dead polymer chains, and of monomer for TEMPOmediated ethene polymerization initiated by homolysis of a peroxide initiator and by self-initiation of the monomer. Rate coefficients are from styrene experiments at $125^{\circ} \mathrm{C}$ and 1 bar. $k_{\mathrm{i}}=2 \cdot 10^{-10} \mathrm{~L} \mathrm{~mol}^{-1} \mathrm{~s}^{-1}, k_{\mathrm{l}}=5 \cdot 10^{-3} \mathrm{~s}^{-1}$.

Further simulations of TEMPO-mediated styrene polymerization, using the complete scheme described in Section 4.1, lead to similar evolutions of species concentrations and molecular weights.

These simulations allow to understand and visualize the mechanism of controlled styrene polymerization and the radical persistent effect via the evolution of concentration of the relevant species. In order to understand the reasons for the failure of control in ethene 
polymerization with TEMPO, the rate coefficients for the system ethene-TEMPO will be included in the model and the consequences will be studied.

\subsubsection{Aspects of control in ethene polymerization in the presence of nitroxide}

The model presented in Section 4.1.1 is used for styrene at $125^{\circ} \mathrm{C}$ and 1 bar with the rate coefficients determined by Schmidt-Naake ${ }^{1}$, e.g. $k_{\mathrm{c}}=9 \cdot 10^{5} \mathrm{~L} \cdot \mathrm{mol}^{-1} \cdot \mathrm{s}^{-1}$ and $k_{\mathrm{d}}=10^{-3} \cdot \mathrm{s}^{-1}$. The rate coefficients for ethene polymerization at $200^{\circ} \mathrm{C}$ and 2000 bar are gradually implemented. Firstly, $k_{\mathrm{p}, \mathrm{E}}=1.2 \cdot 10^{4} \mathrm{~L} \cdot \mathrm{mol}^{-1} \cdot \mathrm{s}^{-1}$ and $k_{\mathrm{t}, \mathrm{E}}=2.26 \cdot 10^{8} \mathrm{~L} \cdot \mathrm{mol}^{-1} \cdot \mathrm{s}^{-1}$ are introduced. The simulations do not show any loss of control of the polymerization reaction. The polydispersity is very low (about 1.10) and the polymer molecular weight increases linearly with monomer conversion. The persistent radical effect is also observed. The thermal decomposition of TEMPO, $k_{\text {decomp }}=7 \cdot 10^{-3} \mathrm{~s}^{-1}$, and spontaneous ethene polymerization, $k_{1}=1.76 \cdot 10^{-15} \mathrm{~L} \cdot \mathrm{mol}^{-1} \cdot \mathrm{s}^{-1}$, do not destroy the persistent radical effect and a perfect control of ethene polymerization is observed.

Reactions of transfer to monomer, $k_{\mathrm{tr}, \mathrm{M}}=1.87 \mathrm{~L} \cdot \mathrm{mol}^{-1} \cdot \mathrm{s}^{-1}$ at $200^{\circ} \mathrm{C}$ and $2000 \mathrm{bar}$, however induce a strong increase of the polydispersity index up to 1.90 . The molar mass of the dormant species increases with monomer conversion. This indicates that, under these conditions, the control of the reaction is partially lost because of transfer to monomer.

The evolution of the species concentrations with reaction time plotted in Fig. 4.18 shows that the radical persistent effect is still effective. Only the variation of the dead polymer concentration differs from the simulation in Fig. 4.17 with low transfer to monomer (data for styrene polymerization at $125^{\circ} \mathrm{C}$ and $1 \mathrm{bar}$ ). The dead polymer concentration increases and reaches a maximum after $10^{3} \mathrm{~s}$. The concentration of dormant species is not influenced and reaches a maximum after about $3 \cdot 10^{2} \mathrm{~s}$. 


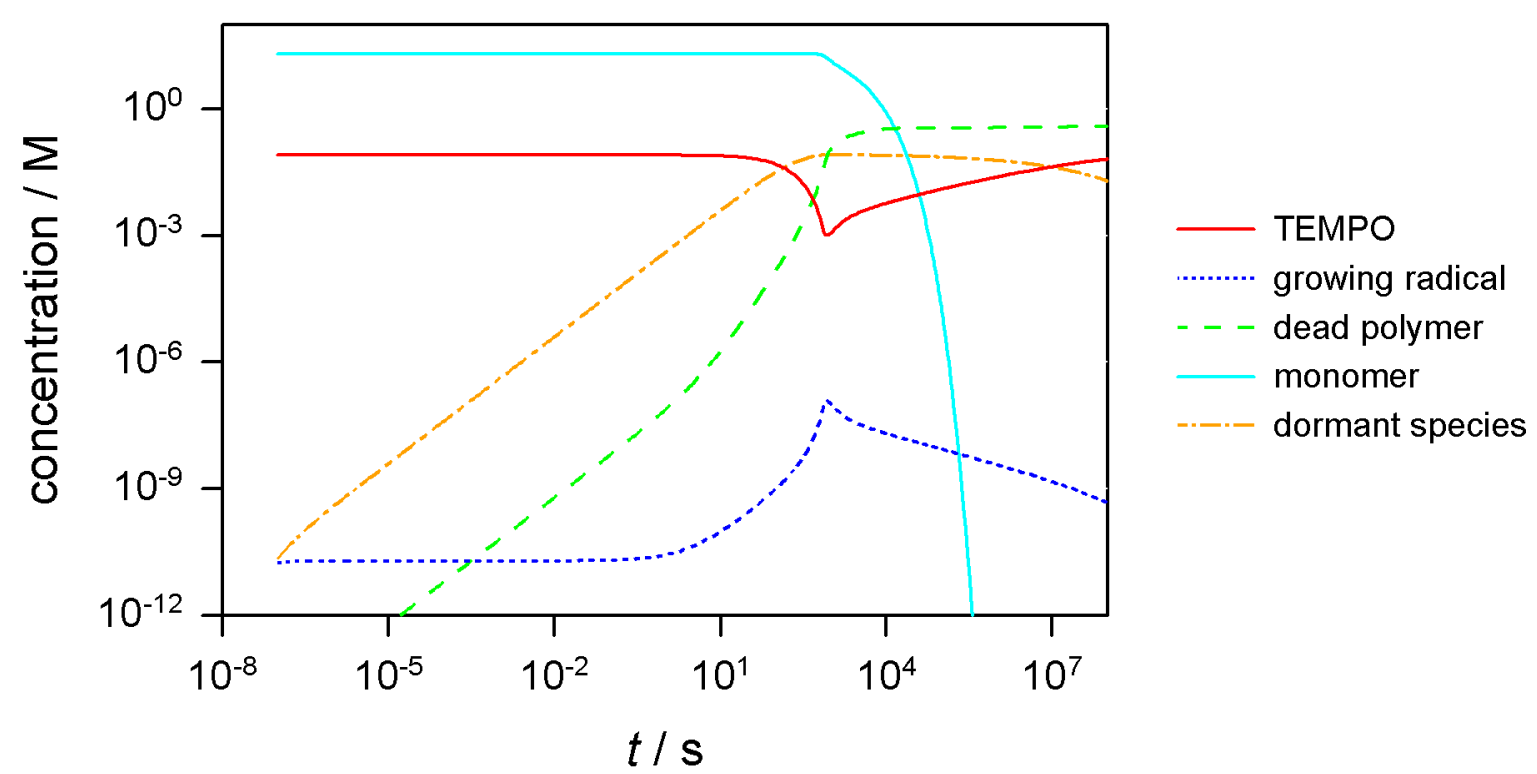

Fig. 4.18 Time evolution of concentration of growing and persistent radicals, of dormant species and of dead polymer chains, and of monomer for TEMPO-mediated ethene polymerization initiated by homolysis of a peroxide initiator and by self-initiation of the monomer. $k_{p}, k_{t}, k_{\text {decomp }}, k_{i}, k_{t r, M}$ refer to ethene at $200^{\circ} \mathrm{C}$ and $2000 \mathrm{bar} ; k_{c}=9 \cdot 10^{5} \mathrm{~L} \cdot \mathrm{mol}^{-1} \cdot \mathrm{s}^{-1}, k_{d}=10^{-3} \mathrm{~s}^{-1}$ are from styrene experiments at $125^{\circ} \mathrm{C}$ and $1 \mathrm{bar}$.

A poor control of the ethene polymerization is observed due to transfer to monomer (at $200^{\circ} \mathrm{C}$ and $2000 \mathrm{bar}$ ). The growing molar mass evolution of the dormant species shows that the polymerization is partially controlled, but with polydispersity indices close to 2 . Polymerizations at lower temperature should allow to reduce transfer to monomer and improve the control of the polymerization.

Finally, the rate coefficients for the reversible deactivation of the growing radical, $k_{\mathrm{c}}$ and $k_{\mathrm{d}}$, were set to values that should applied to ethene polymerization. Combination of nitroxide and alkyl radical is assumed to be of the same rate as is the combination of alkyl radicals (i.e. $\left.k_{\mathrm{c}}=k_{\mathrm{t}}=2.2 \cdot 10^{8} \mathrm{~L} \cdot \mathrm{mol}^{-1} \cdot \mathrm{s}^{-1}\right)$. The insertion of this number into the model does not reduce the control of polymerization.

The value of the rate coefficient of dissociation of the dormant species is deduced from value determined by Fischer for methyl-TEMPO dissociation, reported in Eq. 4.20, to be $k_{\mathrm{d}}=2.7 \cdot 10^{-5} \mathrm{~s}^{-1}$. 
The consequences are illustrated in Fig. 4.19, the free TEMPO concentration undergoes a strong decrease in early period of the polymerization. There is no more any large excess of free TEMPO over the concentration of free radicals, which is essential for control of the polymerization. The persistent radical effect is not effective anymore. The very low persistent radical concentration is due to the very low value of $k_{\mathrm{d}}$ in ethene polymerization compared to the one for styryl-TEMPO at $125^{\circ} \mathrm{C}$. The very high activation energy of $k_{\mathrm{d}}$ expresses the strength of the alkyl-TEMPO bond which strongly shifts the equilibrium to the side of the dormant species.

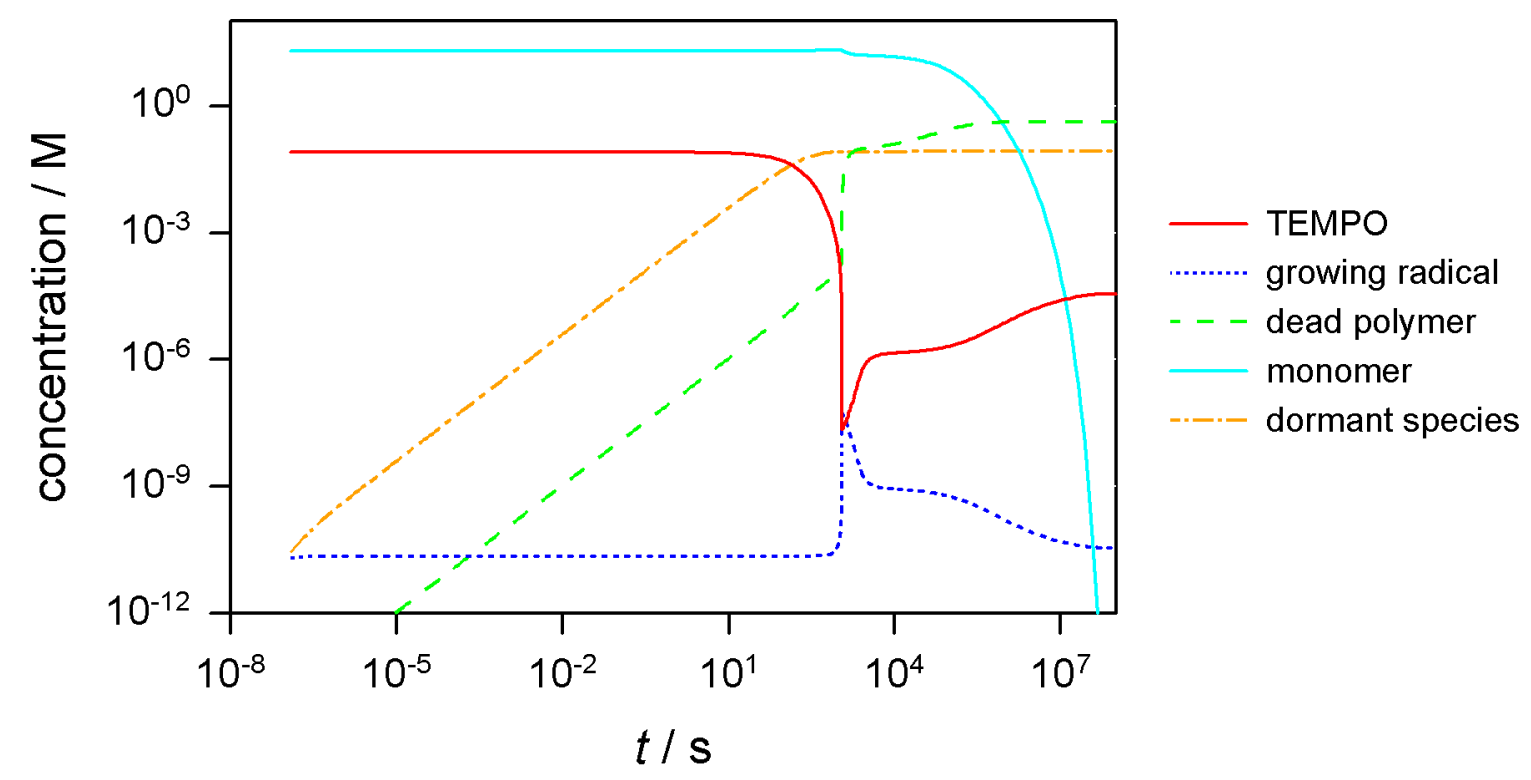

Fig. 4.19 Time evolution of concentration of growing and persistent radicals, of dormant species and of dead polymer chains, and of monomer for TEMPO-mediated ethene polymerization initiated by homolysis of a peroxide initiator and by self-initiation of the monomer. $k_{p}, k_{t}, k_{\text {decomp }}, k_{i}, k_{t r, M}, k_{c}$ and $k_{d}$ refer to ethene polymerization at $200^{\circ} \mathrm{C}$ and 2000 bar.

The molar mass distribution of the dormant species is strongly influenced by the low value of $k_{\mathrm{d}}$. The polydispersity index obtained is much higher than 2 and the weight average molecular weight does not increase linearly with monomer conversion. The $M_{\mathrm{W}}$ obtained is much lower than expected $\left(M_{\mathrm{W} \text { theo }}=140000 \mathrm{~g} \cdot \mathrm{mol}^{-1}\right.$ at $50 \%$ ethene conversion $)$.

Ethene polymerization obviously is difficult to be controlled due to the high stability of the alkyl-TEMPO dormant species. The dissociation of this dormant species requires high 
temperature which, however, induces an important transfer to monomer and significant thermal decomposition of the radical TEMPO.

It will now be tried to find a mediating species that might be suitable for control of ethene polymerization. The following requirements need to met :

1. The dormant species has to be not too stable to allow for the accumulation of a large excess of free nitroxide and then to allow for the persistent radical effect to occur.

2. Pressure and temperature conditions have to be found under which transfer to monomer is tolerable.

The ratio $k_{\mathrm{tr}} / k_{\mathrm{p}}$ may be calculated from literature expressions for the ethene polymerization at 2000 bar, and the values are listed below.

\begin{tabular}{cc}
\hline$\theta /{ }^{\circ} \mathrm{C}$ & $k_{\text {tr }} / k_{\mathrm{p}}$ \\
230 & $3.1 \cdot 10^{-4}$ \\
200 & $1.6 \cdot 10^{-4}$ \\
170 & $7.0 \cdot 10^{-5}$ \\
150 & $3.9 \cdot 10^{-5}$ \\
\hline
\end{tabular}

The ratio $k_{\mathrm{tr}} / k_{\mathrm{p}}$ increases with temperature, which says that transfer to monomer becomes more important at high temperature relative to propagation rate. To avoid the unfavorable action of transfer to monomer, the nitroxide-mediated ethene polymerization has to be carried out at low temperature.

Simulations show that control of ethene polymerization would be possible if the dissociation rate of dormant species is sufficiently high $\left(k_{\mathrm{d}}\right.$ about $\left.10^{-2} \mathrm{~s}^{-1}\right)$ at the lower temperatures. A too stable dormant species, with $k_{\mathrm{d}}$ about $10^{-7} \mathrm{~s}^{-1}$ would induce a loss of controlled polymerization due to the very low persistent radical concentration.

Simulations have been carried out to find $k_{\mathrm{d}}$ values which would allow control of the ethene polymerization. The rate coefficients $k_{\mathrm{d}}$ were calculated for different temperatures such as to obtain a low polydispersity index of 1.10 at $30 \%$ monomer conversion, in the absence of 
transfer to monomer. Tab. 4.20 presents the rate coefficients $k_{\mathrm{d}}$ so obtained and their associated activation energies. Polydispersity indices of dormant species and dead polymer simulated with transfer to monomer are also reported.

\begin{tabular}{lccccc}
\hline$\theta /{ }^{\circ} \mathrm{C}$ & 150 & 170 & 200 & 250 & 300 \\
\hline$k_{\mathrm{d} \text { hexyl-X } / \mathrm{s}^{-1}}$ & $6 \cdot 10^{-3}$ & $5 \cdot 10^{-3}$ & $5 \cdot 10^{-3}$ & $4.5 \cdot 10^{-3}$ & $8 \cdot 10^{-2}$ \\
$E_{\mathrm{a}}{ }^{*} / \mathrm{kJ} \cdot \mathrm{mol}^{-1}$ & 142 & 155 & 160 & 177 & 180 \\
$I p$ dormant species & 1.17 & 1.30 & 1.34 & 1.86 & 1.92 \\
$I p$ dead polymer & 1.58 & 1.48 & 1.42 & 1.95 & 1.91 \\
[dormant species] $/$ & 6.0 & 3.5 & 1.7 & 0.5 & 0.1 \\
[dead polymer] & & & & & \\
\hline
\end{tabular}

*pre-exponential factor: $k_{0}=2.5 \cdot 10^{-15}$

Table 4.20 Estimate of rate coefficient $k_{d}$ of hypothetical dormant species that would allow control of ethene homopolymerization at 2000 bar and at $30 \%$ conversion. $[$ hexyl $-X]=2 \cdot 10^{-3} M$, [ethene $]=20 M$.

The simulations clearly show a better control of polymerization at low temperature. Lower polydispersity indices and higher dormant species concentrations due to lower transfer to monomer are observed. At temperatures up to $200^{\circ} \mathrm{C}$, molecular weight increases with monomer conversion and low dormant species polydispersities are expected. The bond dissociation energy of the hexyl-X has to be sufficiently low in order to control ethene polymerization. The pre-exponential factor has been set to $k_{0}=2.5 \cdot 10^{-15}$. The activation energy of $k_{\mathrm{d}}$ is estimated to be between 140 and $160 \mathrm{~kJ} \cdot \mathrm{mol}^{-1}$.

Simulations were performed to estimate the influence of the ratio $k_{\mathrm{d}} / k_{\mathrm{c}}$ on the polymerization rate and the polydispersity of hypothetically ethene controlled polymerization. In Fig. 4.21 is reported the simulated polydispersity as a function of $k_{\mathrm{d}}$ and $k_{\mathrm{c}}$ rate coefficients. A variation of $k_{\mathrm{c}}$, at constant $k_{\mathrm{d}}$, induces a variation of the polymerization rate and of the polydispersity index, as described in Fig. 4.21. Increasing $k_{\mathrm{c}}$ at constant $k_{\mathrm{d}}$ induces lower polydispersity and lower polymerization rate due to the faster deactivation of growing radicals. At constant ratio $K$ of $k_{\mathrm{d}} / k_{\mathrm{c}}$, increasing $k_{\mathrm{c}}$, and then increasing $k_{\mathrm{d}}$ of the same order of 
magnitude, induces a lower polydispersity, without influencing the polymerization rate. It is then clear that $K$ influences the polymerization rate but the control of the polymerization directly depends on $k_{\mathrm{c}}$ and $k_{\mathrm{d}}$ values.

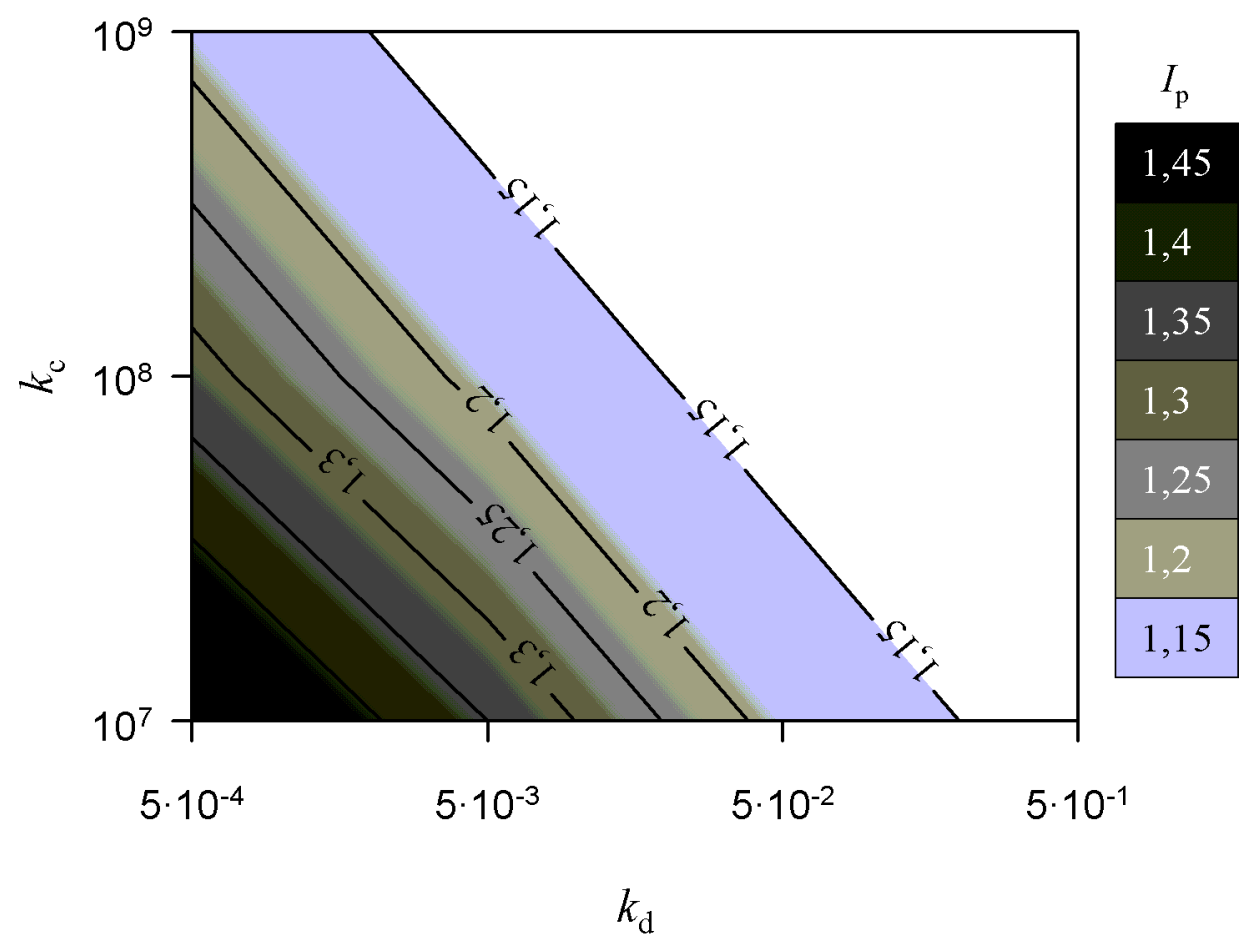

Fig. 4.21 Influence of $k_{c}$ and $k_{d}$ rate coefficients on polydispersity at $30 \%$ conversion of ethene polymerization in the presence of an hypothetical hexyl-adduct. $T=160^{\circ} \mathrm{C}, P=2000$ bar, $[$ hexyl $-X]=2 \cdot 10^{-3} \mathrm{M}$, [ethene $]=20 \mathrm{M}$.

\subsection{References}

[1] G. Schmidt-Naake, M. Drache, C. Han, A. Koch, Dechema 1998, 134,601

[2] K. Matyjaszewski, D. Greszta, Macromolecules 1996, 29, 7661

[3] T. Fukuda, A. Goto, K. Ohno, Y. Tsujii, ACS Symp. 1997, 685, 180

[4] M. Georges, R. Veregin, K. Daimon, ACS Symp. 1997, 685, 170

[5] K. Matyjaszewski, D. Greszta, D. Mardare, Macromolecules 1994, 27, 638

[6] D. Greszta, K. Matyjaszewski, Polym. Prepr. (Am. Chem. Soc., Div. Polym. Chem., 1994, 35 (1), 778

[7] A. Hui, A. Hamilec, J. Appl. Polym. Sci. 1972, 16, 749 
[8] M. Buback, R. Gilbert, R. Hutchinson, F.D. Kuchta, J. Macromol. Chem. Phys. 1995, 196,3267

[9] I. Li, K. Matyjaszewski, T. Shigemoto, P. Smith, D. Priddy, Macromolecules 1996, 29, 5239

[10] M. Buback, B. Huckestein, F.D. Kuchta, G. Russel, E. Schmidt, J. Macromol. Chem. Phys. 1994, 195, 2117

[11] M. Buback, F.D. Kuchta, J. Macromol. Chem. Phys. 1995, 196, 1887

[12] M. Buback, F.D. Kuchta, J. Macromol. Chem. Phys. 1997, 198, 1455

[13] T. Fukuda, T. Terauchi, Chem. Lett. (Tokyo) 1996, 293

[14] T. Fukuda, T. Terauchi, T. Miyamoto, Polym. Prepr. Jpn. 1996, 45, 1261

[15] K. Matyjaszewski, D. Greszta, J. Polym. Sci. Polym. Chem. 1998, 29

[16] S. Agraval, C. Han, A. I. Ch. D. J. 1975, 21, 449

[17] C. Chen, J. Vermeychuk, J. Howel, P. Ehrlich, A. I. Ch. D. J. 1976, 22, 463

[18] H. Kiu Lee, J. Marano, J. Am. Chem. Soc. Symp. 1979, 104, 221

[19] S. Goto, K. Yamamoto, S. Furui, M. Sugimoto, J. Appl. Polym. Sci. : Appl. Polym. Symp. 1981, 36, 21

[20] J. Schweer, Ph. D. Thesis Göttingen 1988

[21] M. Buback, C. Chloe, E. Franck, Macromol. Chem. 1984, 185, 1699

[22] P. Lorenzini, M. Pons, J. Villermaux, Chem. Eng. Sci. 1992, 47, 3981

[23] P. Ehrlich, G. Mortimer, Adv. Polym. Sci. 1970, 1, 86

[24] M. Roedel, J. Am. Chem. Soc. 1953, 75, 6110

[25] A. Willbourn, J. Pol. Sci. 1959, 34, 569

[26] D. Dorman, E. Otocka, F. Bovey, Macromolecules 1972, 5, 574

[27] J. Randall, Polymer Sequence Determination, Academic press, New York 1977

[28] F. Bovey, F. Schilling, F. McCracking, H. Wagner, Macromolecules 1976, 9, 76

[29] M. Cudby, A. Bunn, Polymer 1976, 17, 345

[30] G. Luft, R. Kämpf, H. Seidl, Angew. Makromol. Chem. 1982, 108, 203

[31] R. Mullikin, G. Mortimer, J. Macromol. Sci.-Chem. 1970, A4, 1495

[32] A. Holmström, E. Sörvic, J. Appl. Polym. Sci. 1974, 18, 761

[33] C. Kiparissides, G. Verros, J. McGregor, J. Macromol. Sci.-Rev. Macromol. Chem. Phys. 1993, C33 (4), 437

[34] M. Buback, C. Chloe, E. Franck, Macromol. Chem. 1984, 185, 1685

[35] T. van der Molen, IUPAC Symp. 1969, III, 19 
[36] M. Busch, submitted to Macromol. Theory and Simul.

[37] F. Mähling, R. Klimesch, M. Schwibach, M. Buback, M. Busch, Chem. Ing. Tech. 1999, 71, 1301

[38] H. Fischer, submitted to Chem. Ing. Tech. part 1 and 2

[39] T. Otsu, M. Yoshida, T. Tazaki, Macromol. Rapid. Comm. 1982, 3, 133

[40] K. Matyjaszewski, A. Kajivara, Macromolecules 1998, 31, 548

[41] P. MacLeod, R. Veregin, P. Odell, Macromolecules 1998, 31, 530

[42] T. Fukuda, T. Terauchi, A. Goto, T. Tsujii, S. Kobatake, B. Yamada, Macromolecules 1996, 29, 6393

[43] H. Fischer, Macromolecules 1997, 30, 5666

[44] H. Fischer, J. Polym. Sci. Part A : Polym. Chem. 1999, 37, 1885 


\section{EXPERIMENTAL OBSERVATIONS}

\subsection{IR/NIR-experiments}

The infrared special range extends from 30 to $12000 \mathrm{~cm}^{-1}$. It is devided into three regions; the far infrared (FIR), from 30 to $300 \mathrm{~cm}^{-1}$, the mid infrared (IR) from 300 to 4000 $\mathrm{cm}^{-1}$, and the near infrared (NIR), from 4000 to $12000 \mathrm{~cm}^{-1}$. The FIR spectrum is of little use for determining the composition of fluid systems. IR and NIR are the regions where the fundamental overtone and combination modes of molecular vibrations occur. They contain a wealth of characteristic absorption bands. At appropriately chosen concentration and path length, characteristic absorbances of useful magnitude are readily gained. Accordingly, this region has proven to be extraordinarily suitable for the qualitative and quantitative analysis of liquids, but also of solids and gases ${ }^{1}$. This method finds application in this work, where a characteristic absorbance of the carbonyl group (with TBPA and TAPP) and absorbances of decomposition products (e.g. from DTBP) have been used in quantitative investigations into peroxide decomposition kinetics.

The decrease of overall monomer concentration, $[\mathrm{M}]$, is monitored via the associated NIR absorption. It has become standard practice to use the first overtone of the $\mathrm{CH}$-vibration at the double bond for quantitative analysis of vinyl monomers. A halogen lamp and an InSb detector have been used for the study. The position of the observed absorption maxima of the $\mathrm{CH}$ vibration at the double bond are summarized for the different monomers in the following table.

\begin{tabular}{ccccc}
\hline & ethene & styrene & butyl acrylate & methyl methacrylate \\
$P$ & $2000 \mathrm{bar}$ & $1 \mathrm{bar}$ & $1 \mathrm{bar}$ & $1 \mathrm{bar}$ \\
$v_{\mathrm{C}-\mathrm{H}(\max )}$ & $6138 \mathrm{~cm}^{-1}$ & $6135 \mathrm{~cm}^{-1}$ & $6170 \mathrm{~cm}^{-1}$ & $6169 \mathrm{~cm}^{-1}$ \\
\hline
\end{tabular}

Integration of the monomer half band allows for the calculation of overall monomer concentration and thus for the kinetic analysis. Bulk polymerization presents the advantage that no overlapping solvent band disturbs the calculation of monomer concentration. 
In Figure 5.1 are shown typical spectra of the monomers collected for the different homopolymerizations in the optical high pressure cell at 2000 bar.

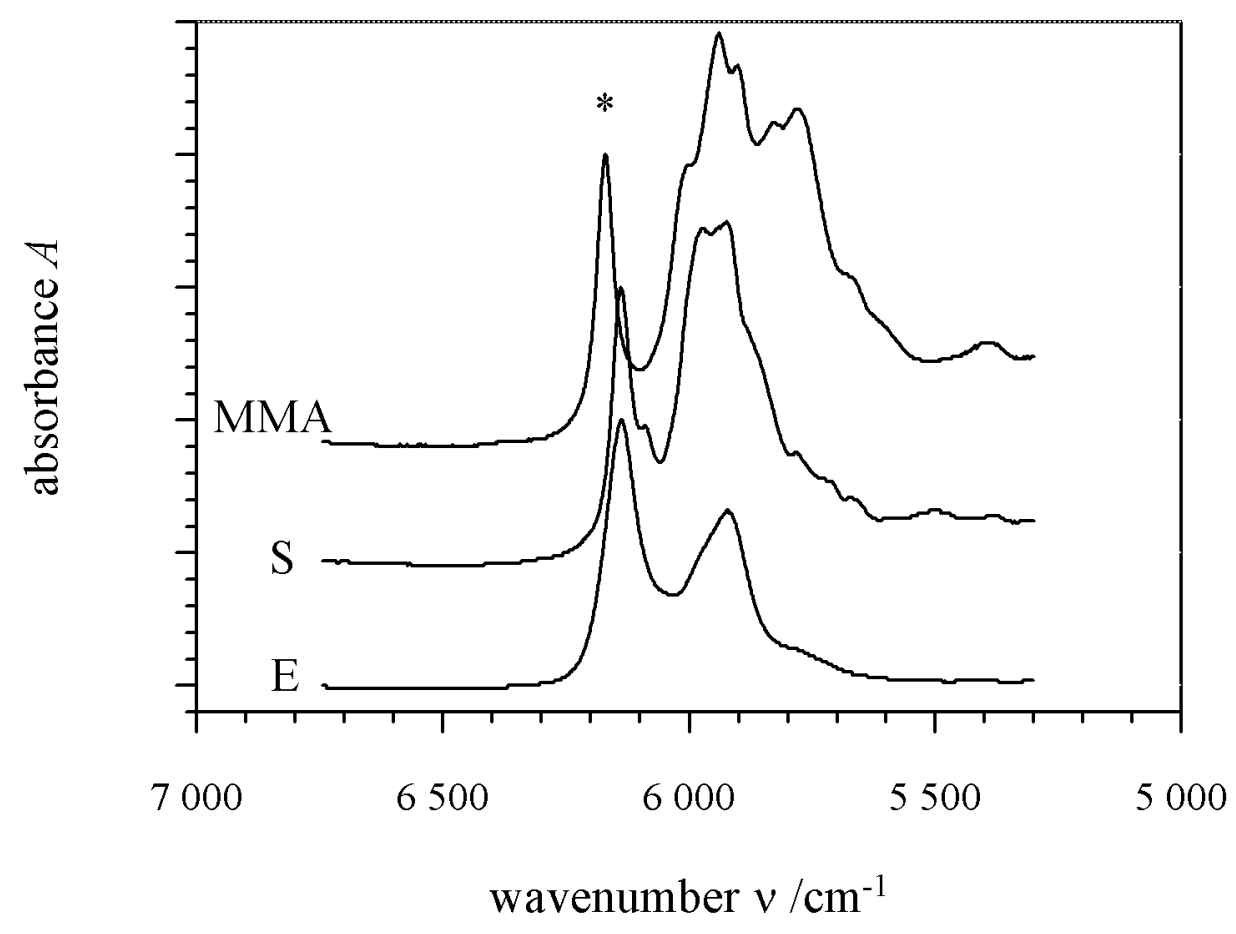

Fig. 5.1. NIR spectra of the different monomers under investigation at 2000 bar. The peak marked with the asterisk is used for the determination of the overall monomer concentration. Its position shifts with experimental conditions.

\subsection{Study of the initiation step}

\subsubsection{Peroxide decomposition in $n$-heptane}

To accurately determine the decomposition rate coefficients and the associated activation parameters, experiments have been carried out in an extended temperature and pressure range. For the continuous flow reactor, the available temperature is determined by the residence time limits. For the reactor used, of length $10.0 \mathrm{~m}$, residence times between 5 and $300 \mathrm{~s}$ allow accurate detection of peroxide concentrations. Hence temperatures must be chosen such that conversions of approximately 5 and $100 \%$ are reached within these residence time limits. 
The different mechanisms of peroxyesters decomposition and their influence on the rate coefficients have already been reported ${ }^{7-11}$. They are given in scheme 5.2 . The peroxide can undergo a simple bond scission (1), producing two oxygen centered free radicals (as with $\mathrm{TBPA}^{7}$ ) or a concerted two-bond scission (2) with the simultaneous rupture of the alkyl- $\mathrm{CO}_{2}$ bond (case of TAPP) and release of $\mathrm{CO}_{2}$. Kharasch and Fono were the first to discover that metal salts catalyze the decomposition of peroxides (3) ${ }^{12}$, but now it is well known that many metals and their salts accelerate peroxide decomposition ${ }^{13}$. However, it seems that, with the continuous reactor, at high temperatures side reactions such as metal catalysis are less important ${ }^{14}$. Other groups ${ }^{15-16}$ who used steel autoclaves for investigations into the decomposition of diacyl peroxides at high temperatures did not consider catalytic activity of the wall material.
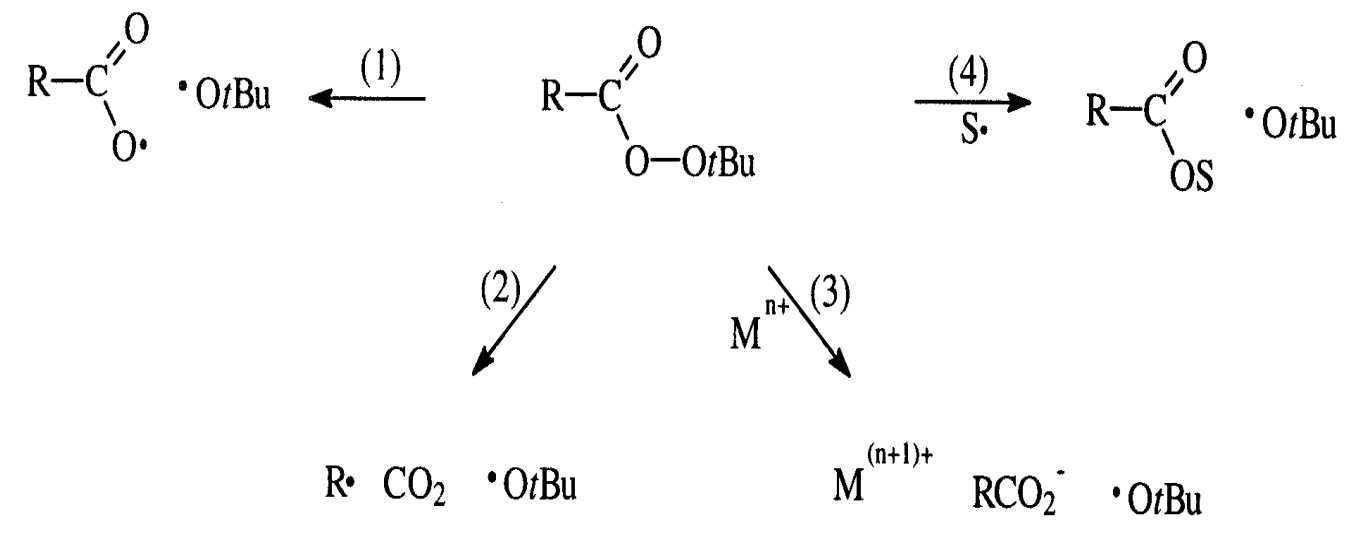

Scheme 5.2. Different mechanisms involved in peroxide decomposition.

The decomposition of TAPP in $n$-heptane was investigated spectroscopically over the temperature range 120 to $140^{\circ} \mathrm{C}$ at 500 bar.

In Figure 5.3 is shown a typical series of spectra collected during the course of the decomposition of TAPP in $n$-heptane carried out at $120^{\circ} \mathrm{C}$ in the continuous flow apparatus. The wavenumber range is from 1650 to $1850 \mathrm{~cm}^{-1}$, where the $\mathrm{C}=\mathrm{O}$ fundamental absorbs. The spectra show the distinctive signal of the peroxide which disappears with time. The IR signal $\left(v_{\mathrm{C}=\mathrm{O}}\right.$ stretching mode) of the peroxide has a maximum at $1770 \mathrm{~cm}^{-1}$. Also apparent on the lower frequency is the growing peak of a carbonyl containing decomposition product (acetone at $\left.1720 \mathrm{~cm}^{-1}\right)$. 


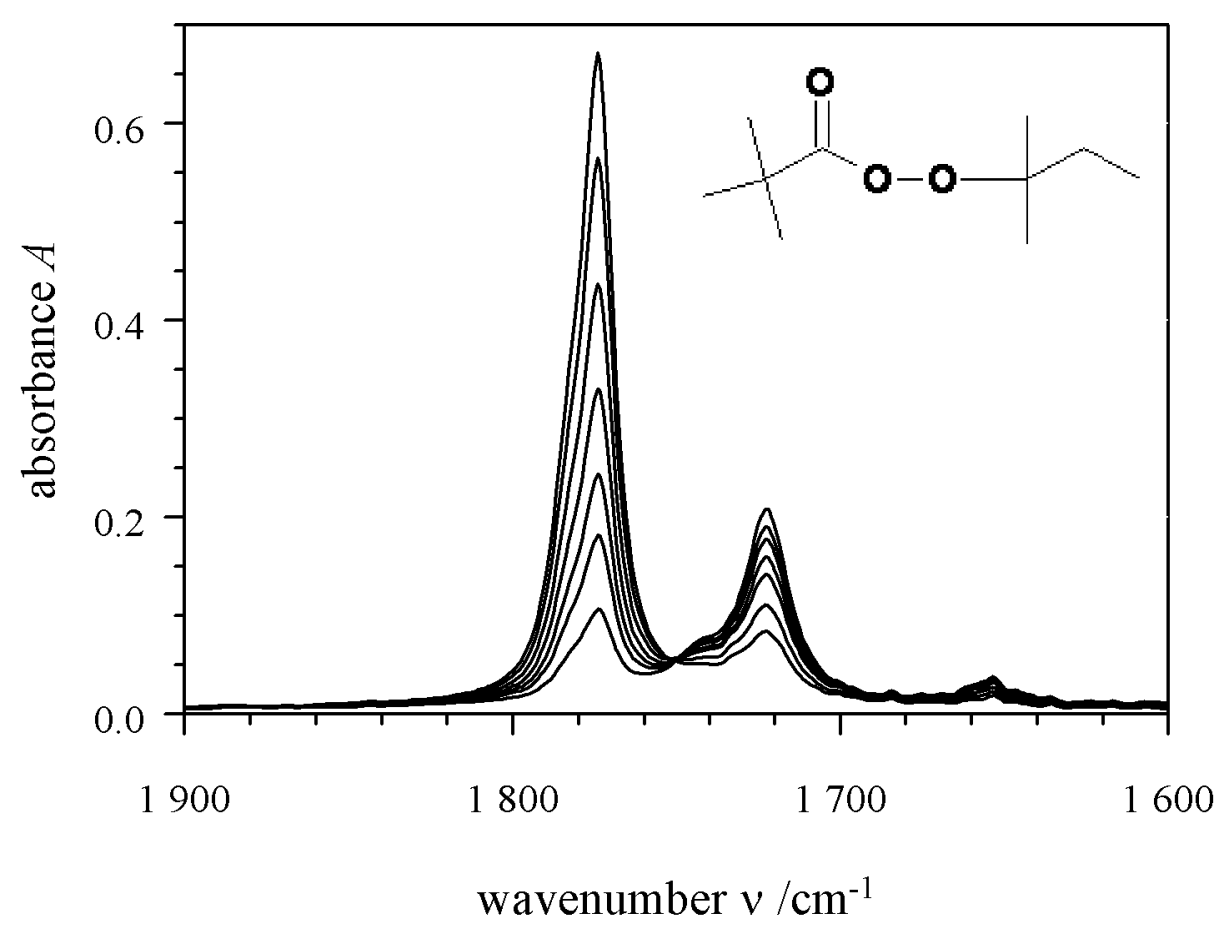

Fig. 5.3. Spectral series of the decomposition of TAPP in $n$-heptane $\left(120^{\circ} \mathrm{C}, 500\right.$ bar, $0.01 \mathrm{M}$, optical path length : $1.2 \mathrm{~mm}$ ).

The decomposition of DTBP in $n$-heptane has been investigated over the temperature range from 160 to $210^{\circ} \mathrm{C}$ at $500 \mathrm{bar}$, and from 500 to 2500 bar at $200^{\circ} \mathrm{C}$. The decomposition of dialkyl peroxides such as DTBP can not be spectroscopically investigated by monitoring the diminishing peroxide concentration as no suitable IR-signal for DTBP is available. DTPB decomposition is investigated by observing the absorbance of decomposition products, such as acetone $\mathrm{AC}$ and tert-butanol TBA. The absorbance of these products is lower than the one of the carbonyl stretching mode of peroxides. Thus a solution of higher peroxide concentration needs to be used (DTBP in $n$-heptane $0.1 \mathrm{M}$ ).

In Figure 5.4, is shown a typical series of spectra collected during the course of the decomposition of DTBP in $n$-heptane carried at $200^{\circ} \mathrm{C}$. The spectra show the distinct signals of the products of peroxide decomposition which develop with time. The acetone peak maximum $\left(v_{\mathrm{C}=\mathrm{O}}\right.$ stretching mode) occurs at around $1725 \mathrm{~cm}^{-1}$ and the TBA $\left(v_{\mathrm{OH}}\right.$ stretching mode) is located at $3625 \mathrm{~cm}^{-1}$. 


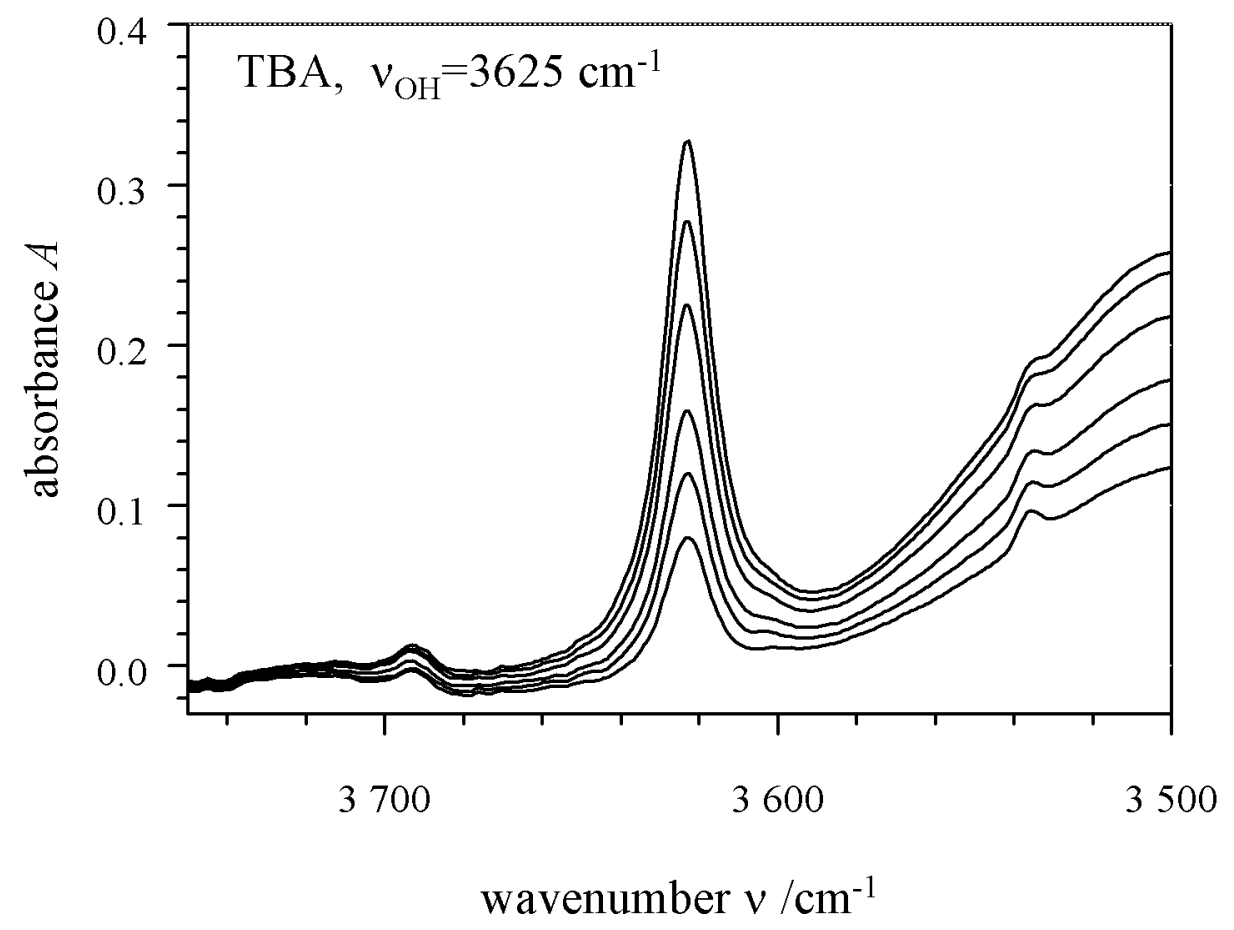

Fig. 5.4. Spectral series of tert-butanol issued from the decomposition of DTBP in nheptane $\left(200^{\circ} \mathrm{C}, 500\right.$ bar, $0.1 \mathrm{M}$, optical path length : $\left.1.2 \mathrm{~mm}\right)$.

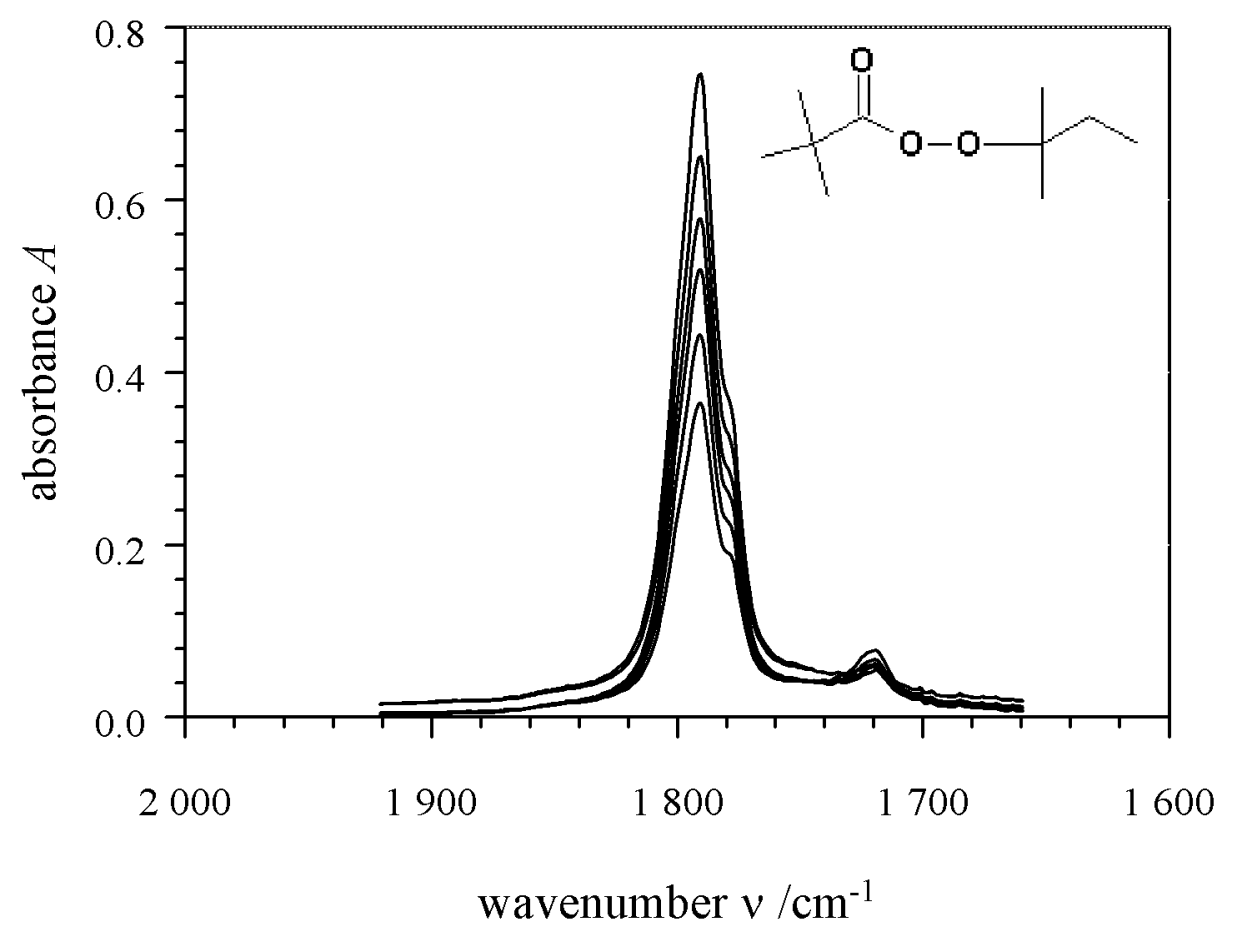

Fig. 5.5. Spectral series of the decomposition of TBPA in $n$-heptane $\left(160^{\circ} \mathrm{C}, 1000\right.$ bar, $0.01 \mathrm{M}$, optical path length : $1.2 \mathrm{~mm}$ ).

The decomposition of TBPA in $n$-heptane was investigated spectroscopically over the temperature range $160-180^{\circ} \mathrm{C}$ at 1000 bar. In Figure 5.5 is shown a typical series of spectra 
collected during the course of the decomposition of TBPA in $n$-heptane carried out at $160^{\circ} \mathrm{C}$ and 1000 bar in the continuous flow apparatus. The IR signal of the peroxide $v_{\mathrm{C}=\mathrm{O}}$ stretching mode has maximum absorbance at $1770 \mathrm{~cm}^{-1}$. The absorbance of decomposition products from TBPA were also observed. The IR signal at $1717 \mathrm{~cm}^{-1}$ is due to acetic anhydride ${ }^{2-3}$.

\subsubsection{Peroxide decomposition in the presence of TEMPO in $n$-heptane}

TEMPO has no characteristic absorbance in the spectroscopic range under investigation ( $\mathrm{v}$ above $1600 \mathrm{~cm}^{-1}$ ). Thus prevents spectroscopic determination of TEMPO concentration. On the other hand, TEMPO does not interfere with signals of the peroxides or of any decomposition products. The same peroxides as before (see section 5.2.1) were investigated in the presence of TEMPO. TAPP and TBPA (0.01M) decompositions were carried out for a ratio of $[$ TEMPO $] /[$ peroxide $]=2$.

Decomposition at ratios of $[$ TEMPO $] /[\mathrm{DTBP}]=0.6$ and 2 have been investigated in the case of the DTBP decomposition at different temperatures. Spectra in the presence of TEMPO are similar to those previously reported without nitroxide. An example of a spectral series of TBPA decomposition in the presence of TEMPO is plotted in Fig. 5.6.

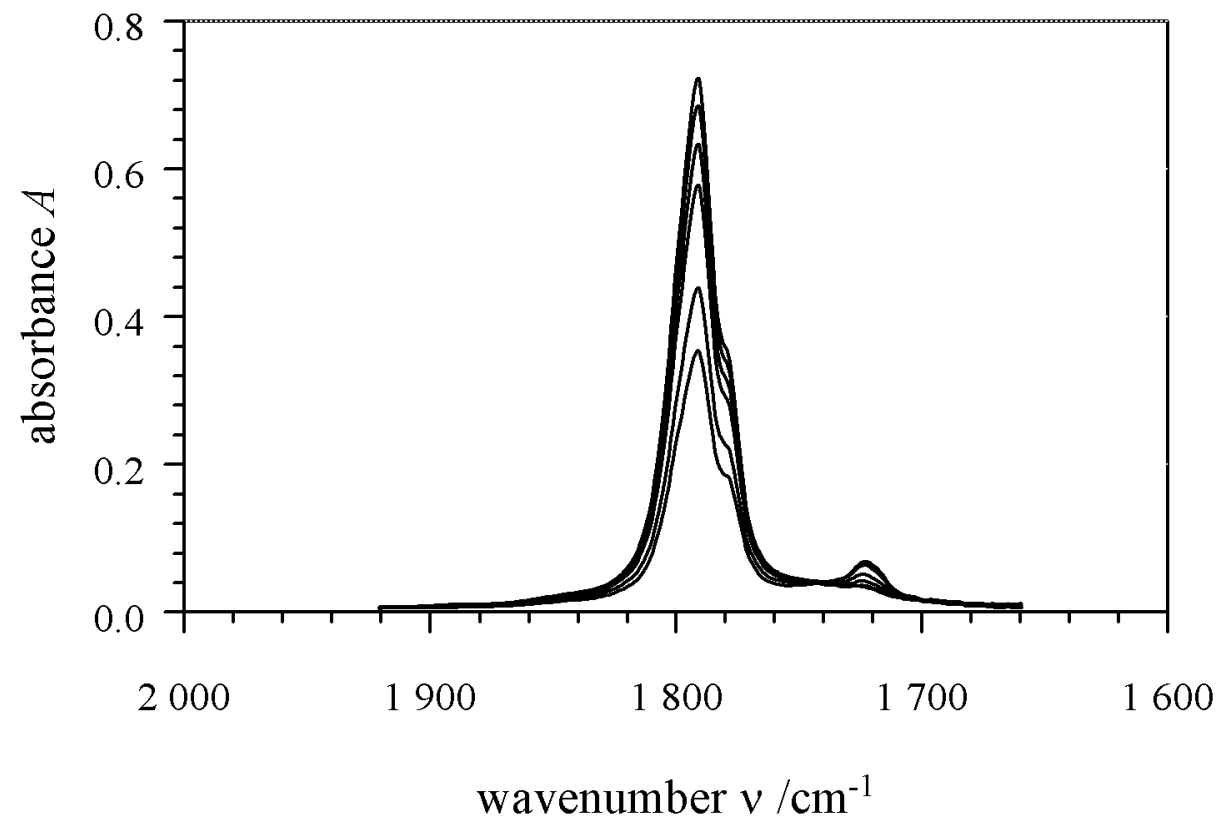

Fig 5.6. Spectral series of the decomposition of TBPA in n-heptane in the presence of TEMPO $\left(160^{\circ} \mathrm{C}, 1000\right.$ bar, $0.01 \mathrm{M},[\mathrm{TEMPO}] /[\mathrm{TBPA}]=2$, optical path length : $1.2 \mathrm{~mm})$. 
In the presence of TEMPO, gaseous decomposition products, most likely methane and ethane, are not observed contrary to what is formed in thermal decomposition without TEMPO.

\subsubsection{Quantitative IR Spectroscopy}

Quantitative analysis of the absorbance spectra proceeds via the Beer-Lambert law, which says that absorbance $A$ is proportional to the optical path length $l$ and the concentration $c$ of an absorbing species. $A$ is given by the decadic logarithm of the intensity of the incident radiation, $I_{0}$, divided by the transmitted radiation, $I$. The proportionality factor $\varepsilon(v)$ is referred as molar absorption coefficient.

$$
A(v)=\log \frac{I_{0}(v)}{I(v)}=\varepsilon(v) \cdot c \cdot l
$$

The quantity $\varepsilon(v)$ exhibits a strong temperature and pressure dependence. For this reason, quantitative analysis is best afforded using integrated absorbance bands, as band areas are little influenced by temperature and pressure. Quantitative evaluation of absorption spectra is thus affected via the integrated form of the Beer-Lambert law :

$$
\text { Int }=\int A(v) \cdot d v=c \cdot l \cdot \int \varepsilon(v) \cdot d v=c \cdot l \cdot B(v)
$$

where Int is the integrated absorbance and $B$ is the integrated molar absorptivity.

\subsubsection{Evaluation of first-order rate coefficients}

Instabilities of the spectrometer and fluctuations of the purge way may shift the baseline for a series of spectra during the course of the experiment. For this reason, absorbance spectra were baseline-corrected via inspection of the wavenumber region where neither peroxide nor the products absorb. 
The first-order reaction of a species is described by the integrated rate equation

$$
\ln \frac{c_{\mathrm{t}}}{c_{0}}=-k \cdot t
$$

where $k$ is the first order rate coefficient, $c_{0}$ is the initial concentration of the peroxide, and $c_{\mathrm{t}}$ is the concentration at a given time $t$. According to the Beer-Lambert law, the integrated characteristic absorbance of a species is proportional to its concentration, such that

$$
\ln \frac{c_{\mathrm{t}}}{c_{0}}=\ln \frac{A_{\mathrm{t}}}{A_{0}}
$$

where $A_{0}$ is the initial integrated absorbance of the substance and $A_{\mathrm{t}}$ the integrated absorbance at time $t$.

Combining equations 5.3 and 5.4, yields

$$
\ln \frac{A_{\mathrm{t}}}{A_{0}}=-k \cdot t
$$

As has been outlined in the previous section, for experiments performed in the continuous apparatus, the time scale is expressed in terms of residence time $\tau$. Each spectrum corresponds to a distinct residence time. Hence the integrated absorbance spectra for the decomposition allows for evaluation of first-order rate coefficients by plotting $\ln \left(A_{t} / A_{0}\right)$ against residence time. The slope of the straight line so obtained yields the rate coefficient $k$.

In Figure 5.7 is shown a typical plot of $\ln \left(A_{t} / A_{0}\right)$ versus residence time for the decomposition of TAPP in $n$-heptane at 500 bar and temperatures from 120 to $135^{\circ} \mathrm{C}$. Fig 5.7 demonstrates that at each temperature the peroxide decomposition follows first-order kinetics. 


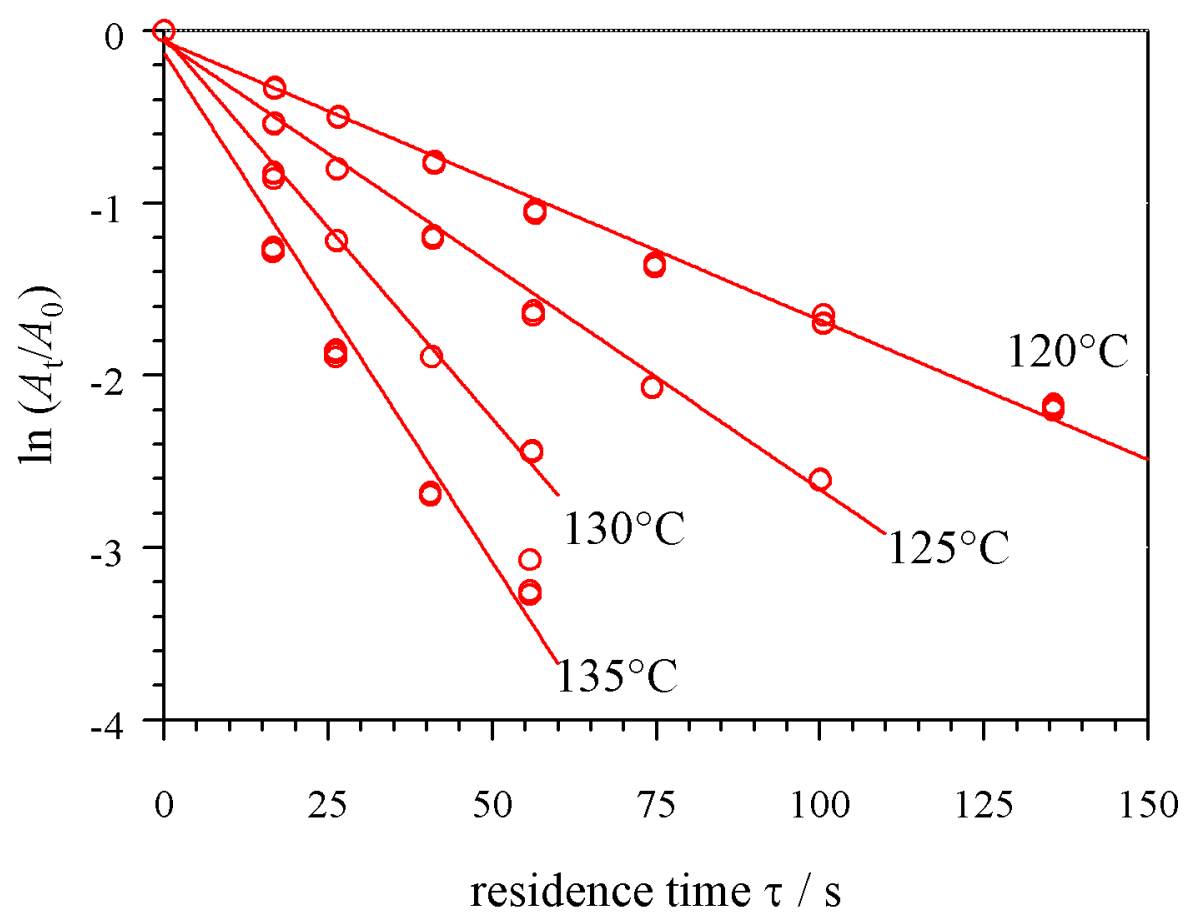

Fig. 5.7. First order $\ln \left(A_{t} / A_{0}\right)$ vs residence time plots for the decomposition of TAPP in n-heptane $\left(120-135^{\circ} \mathrm{C}, 500\right.$ bar, $\left.0.01 \mathrm{M}\right)$.

Plots of $\ln \left(A_{t} / A_{0}\right)$ versus residence time were constructed for each TAPP and TBPA decomposition experiments at constant $T$ and $P$. This method may not be applied with DTBP decomposition.

A first-order rate law has also been used to fit DTBP decomposition in $n$-heptane :

$$
\frac{d c_{D T B P}}{d t}=-k_{o b s} \cdot c_{D T B P}
$$

Tert-butanol (TBA) and acetone (AC) are the only oxygen-containing products that are identified from IR and GC analysis in the samples of this study. Assuming TBA and AC to be produced by parallel first-order reactions, characterized by rate coefficients $k_{\mathrm{TBA}}$ and $k_{\mathrm{AC}}$, respectively, allows to derive Eq. 5.7. By assuming that the fraction of DTBP that reacts to TBA, $k_{\mathrm{TBA} /(} k_{\mathrm{TBA}+} k_{\mathrm{AC})}$, stays constant during a particular DTBP decomposition reaction, even without knowing a number for this ratio, $k_{\mathrm{obs}}$ is directly accessible from the spectroscopically measured TBA concentration, $c_{\mathrm{TBA}}$, via Eq. $5.7^{3}$.

$$
c_{T B A}=c_{T B A}^{\infty}-2 c_{D T B P}^{0} \frac{k_{T B A}}{k_{T B A}+k_{A C}} \cdot \exp \left(-k_{o b s} \cdot t\right)
$$


where $c_{\mathrm{TBA}}^{\infty}$ and $c_{D T B P}^{0}$ refer to TBA concentration after complete decomposition and to initial DTBP concentration, respectively. The overall decomposition rate coefficients, $k_{\mathrm{obs}}$, is found by non-linear regression (using the Levenberg-Marquard algorithm) of the spectroscopically measured TBA concentration vs time data. An important advantage of the non-linear regression relates to the fact that a constant absorbance background, e.g. due to solvent absorbance is considered in the fitting procedure ${ }^{4}$, which therefore does not interfere the analysis for $k_{\text {obs. }}$

In Figure 5.8 is shown a typical graph of $A_{\mathrm{t}}\left(\mathrm{v}_{\mathrm{OH}}\right.$ stretching of TBA at $\left.3625 \mathrm{~cm}^{-1}\right)$ versus residence time for the decomposition of DTBP in $n$-heptane at 500 bar and at temperatures of 170 to $200^{\circ} \mathrm{C}$.

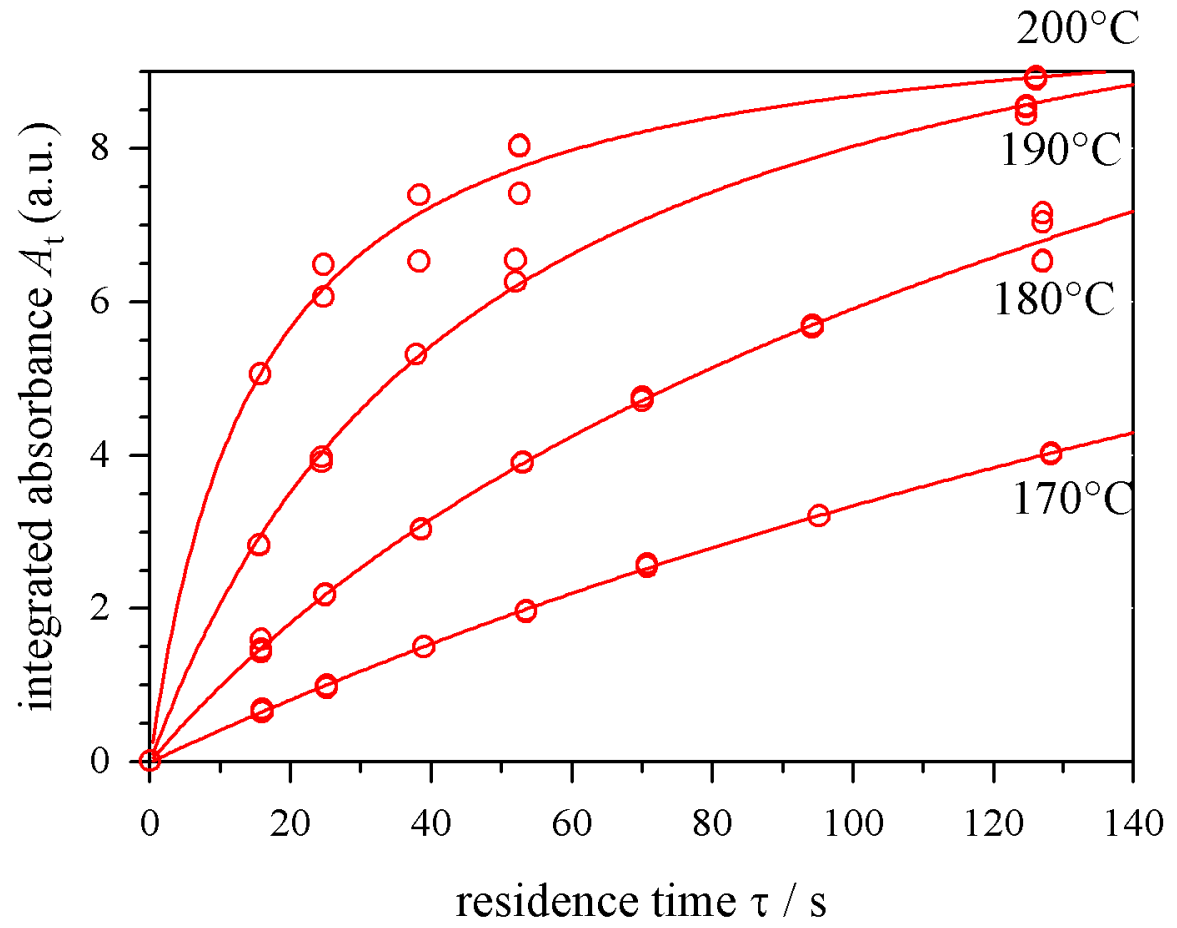

Fig. 5.8. Integrated absorbance $A_{t}$ of the $O H$ stretching mode of TBA at $3625 \mathrm{~cm}^{-1}$ measured as a function of residence time $\tau$ during the decomposition of DTBP in n-heptane $\left(170-200^{\circ} \mathrm{C}, 500 \mathrm{bar}, 0.1 \mathrm{M}\right)$.

\subsubsection{Tabulated first-order rate coefficients}

In the following sections are listed the first-order rate coefficients for the decomposition of the peroxides under investigation. For each tabulated set of results, the 
method of evaluation is given as either (1) determined by peroxide absorbance or (2) determined by product absorbance.

The accuracy of the $k$ values is estimated to be better than $\pm 10 \%$.

$\underline{\text { Decomposition of TAPP }}$

Method of evaluation : (1)

Pressure : 500 bar

$[\mathrm{TAPP}]=0.01 \mathrm{M}$

\begin{tabular}{|c|c|c|c|}
\hline \multirow{2}{*}{$\theta /{ }^{\circ} \mathrm{C}$} & \multicolumn{3}{|c|}{$k_{\mathrm{obs}} / 10^{-3} \mathrm{~s}^{-1}$ (high wavenumber of TAPP) } \\
\cline { 2 - 4 } & \multicolumn{2}{|c|}{$[$ TEMPO]/[TAPP] $=0$} & {$[$ TEMPO]/[TAPP] $=2$} \\
\hline 120 & 20.5 & $19.8^{*}$ & 22.1 \\
125 & 32.2 & - & 33.1 \\
130 & 50.9 & $50.5^{*}$ & 52.5 \\
135 & 78.9 & - & 81.8 \\
140 & - & $130^{*}$ & 133 \\
\hline
\end{tabular}

* literature data ${ }^{5}$

$\underline{\text { Decomposition of DTBP }}$

Method of evaluation : (2)

Pressure : 500 bar

$[\mathrm{DTBP}]=0.1 \mathrm{M}$

\begin{tabular}{|c|c|c|c|c|}
\hline$\theta /{ }^{\circ} \mathrm{C}$ & \multicolumn{4}{|c|}{$k_{\mathrm{obs}} / 10^{-3} \mathrm{~s}^{-1}$ (high wavenumber of TBA) } \\
\cline { 2 - 5 } & {$[$ TEMPO]/[DTBP] $=0$} & {$[$ TEMPO]/[DTBP] $=0.6$} & {$[$ TEMPO]/[DTBP] = 2 } \\
\hline 170 & - & - & 0.9 & - \\
180 & 4.9 & $4.1^{*}$ & 5.6 & 8.0 \\
190 & 9.6 & $9.6^{*}$ & 9.7 & 19.7 \\
200 & 24.4 & $22.0^{*}$ & 23.9 & 32.5 \\
210 & 51.0 & - & 50.5 & 58.0 \\
\hline
\end{tabular}

* literature data ${ }^{3}$ 
Temperature : $200^{\circ} \mathrm{C}$

$[\mathrm{DTBP}]=0.1 \mathrm{M}$

\begin{tabular}{|c|c|c|}
\hline \multirow{2}{*}{$P /$ bar } & $k_{\text {obs }} / 10^{-3} \mathrm{~s}^{-1}$ (high wavenumber of TBA) \\
\cline { 2 - 3 } & {$[$ TEMPO]/[DTBP] $=0$} & {$[$ TEMPO]/[DTBP] $=2$} \\
\hline 500 & 22.4 & 36.4 \\
1000 & 19.2 & 22.4 \\
1500 & 17.0 & 15.4 \\
2000 & 15.1 & 10.7 \\
2500 & 14.2 & 8.9 \\
\hline
\end{tabular}

$\underline{\text { Decomposition of TBPA }}$

Method of evaluation : (1)

Pressure : 1000 bar

$[\mathrm{TBPA}]=0.01 \mathrm{M}$

\begin{tabular}{|c|c|c|}
\hline \multirow{2}{*}{$/{ }^{\circ} \mathrm{C}$} & \multicolumn{2}{|c|}{$k_{\text {obs }} / 10^{-3} \mathrm{~s}^{-1}$ (high wavenumber of TBPA) } \\
\cline { 2 - 3 } & {$[$ TEMPO]/[TBPA] $=0$} & {$[$ TEMPO] $/[$ TBPA] $=2$} \\
\hline 160 & 6.4 & 6.7 \\
165 & 9.5 & 8.6 \\
170 & 14.9 & 15.0 \\
175 & 23.3 & 24.0 \\
180 & 39.7 & 37.6 \\
\hline
\end{tabular}

\subsection{Nitroxide-mediated styrene polymerization}

The high-pressure bulk polymerizations were performed according to the procedures described in Section 3.6.2. Styrene polymerizations were mostly carried out at a common temperature of $125^{\circ} \mathrm{C}$ which is a higher limiting temperature for experiment with $\mathrm{SG} 1$ and is a lower limiting temperature for experiments with DPAIO. This temperature allows for a 
satisfactory polymerization rate with the different nitroxides under investigation. The use of an alkoxyamine as the mediator has been preferred over the combination nitroxide plus thermal initiator, in order to avoid an independent initiation step. In fact, an alkoxyamine, for initiation and control of polymerization, avoids application of a chemical initiator, the initiation efficiency of which is mostly not precisely known.

The integration of the styrene IR band around $6139 \mathrm{~cm}^{-1}$ (at $125^{\circ} \mathrm{C}$ and $2000 \mathrm{bar}$ ) allows to monitor styrene concentration during the polymerization reaction. Kinetics are presented as semilogarithmic plots of $\ln \left([M]_{0} /[M]\right)$ vs reaction time, where $[M]_{0}$ is the initial and $[M]$ the actual monomer concentration. The obtained polymers were studied by GPC to yield molecular weight distribution and polydispersity. The dependence of both these quantities on the degree of monomer conversion will be presented. Experimental initiator concentrations are defined as molar fractions $[\mathrm{I}] /[\mathrm{S}]$ where $[\mathrm{I}]$ and $[\mathrm{S}]$ refer to initiator and styrene molar concentrations, respectively.

\subsubsection{Styrene CRP with SG1}

Fig. 5.9 shows the time dependence of $\ln \left([M]_{0} /[M]\right)$ measured at 60,1000 and 2000 bar and $125^{\circ} \mathrm{C}$ for styrene polymerization in the presence of $0.11 \mathrm{~mol} \%$ styryl-SG1. As was expected from equation 1.1, polymerization is faster at higher pressure. Fig 5.9 shows that the $\ln \left([M]_{0} /[M]\right)$ versus time correlation is not linear but exhibits a downward curvature. The experimental curves are fitted, by a non linear regression, to a function of the type $a+b \cdot t^{c}$. The values of the exponent $\mathrm{c}$ are $0.64,0.69$ and 0.69 for the experiments at 60, 1000 and 2000 bar, respectively. Correlation coefficients are close to 0.999 . The non-linearity of the $\ln \left([M]_{0} /[M]\right)$ vs time plot observed in Fig. 5.9 indicates the minor contribution of termination in styrene CRP in the presence of SG1. The conversion index $\ln \left([M]_{0} /[M]\right)$ seems to follow the rate law deduced by Fischer (see Equation 2.9), where $\mathrm{c}$ is $2 / 3$. 


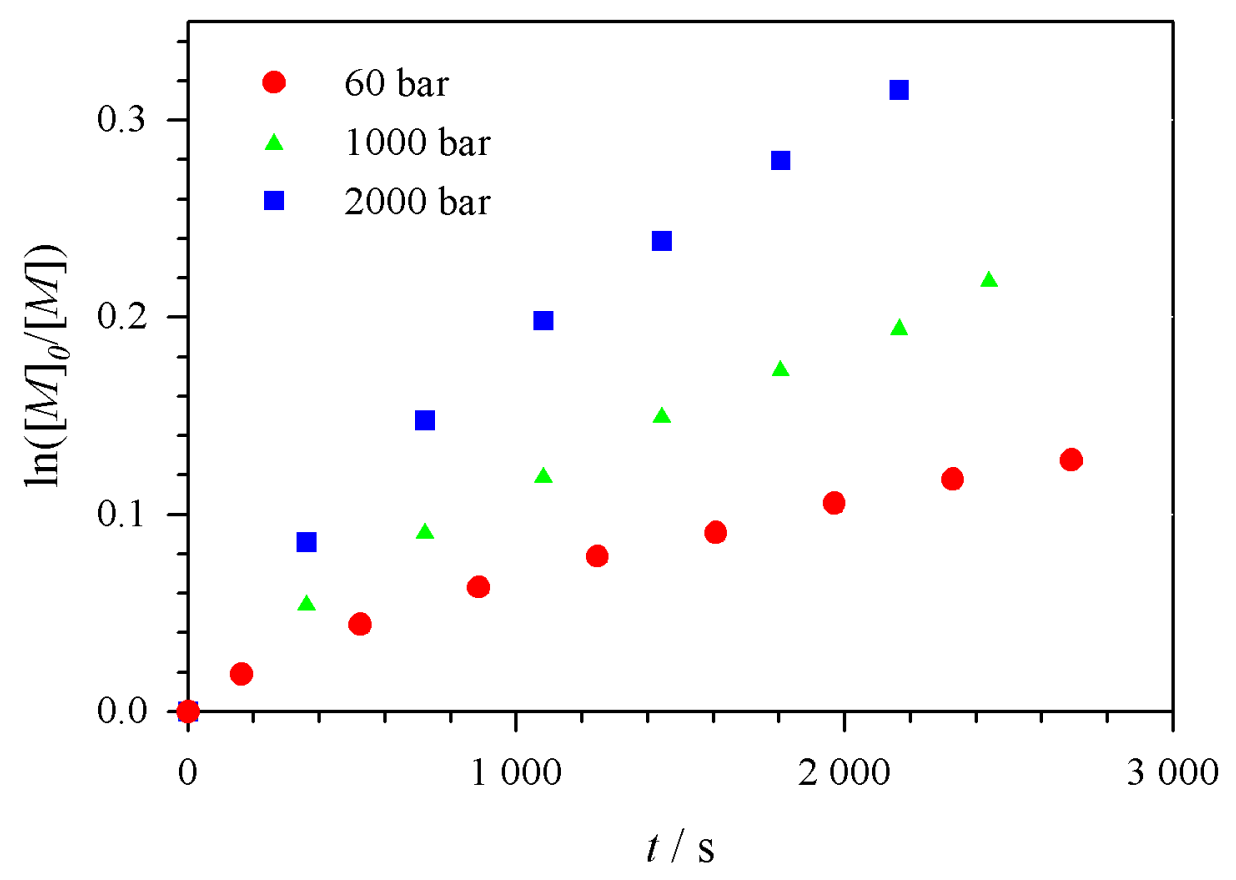

Fig. 5.9. $\ln ([M] d[M])$ plotted vs time for bulk styrene polymerizations in the presence of 0.11 mol\% styryl-SG1, at $125^{\circ} \mathrm{C}$ and three pressures : 60, 1000 and 2000 bar.

GPC measurements on polymer samples for SG1-mediated styrene polymerizations carried out up to different degrees of monomer conversion at $125^{\circ} \mathrm{C}$ and at pressures up to 2000 bar yield the following number average molecular weights, $M_{\mathrm{N}}$, and polydispersity indices, $I p=M_{\mathrm{W}} / M_{\mathrm{N}}$, reported in Table 5.10 and plotted in Fig. 5.11. The linear increase of $M_{\mathrm{N}}$ with monomer conversion and the low polydispersities, around 1.3 at conversions above $10 \%$, demonstrate that SG1 controls styrene polymerization at $125^{\circ} \mathrm{C}$ even under high pressure. The molar mass evolution with conversion does not pass through the origin, probably because of the poorer control at low conversion. Nevertheless, satisfactory polydispersities are obtained at higher conversion, irrespective of the applied pressure. 


\begin{tabular}{ccc|ccc|ccc}
\hline \multicolumn{3}{c|}{$60 \mathrm{bar}$} & \multicolumn{3}{|c|}{$1000 \mathrm{bar}$} & \multicolumn{3}{c}{$2000 \mathrm{bar}$} \\
\hline $\begin{array}{c}\text { conversion } \\
\%\end{array}$ & $\begin{array}{c}M_{\mathrm{N}} \\
\mathrm{g} \cdot \mathrm{mol}^{-1}\end{array}$ & $\mathrm{Ip}$ & conversion & $M_{\mathrm{N}}$ & $I p$ & conversion & $M_{\mathrm{N}}$ & $I p$ \\
$\%$ & $\mathrm{~g} \cdot \mathrm{mol}^{-1}$ & & $\%$ & $\mathrm{~g} \cdot \mathrm{mol}^{-1}$ & \\
\hline 4.8 & 20800 & 1.50 & 9.9 & 35600 & 1.48 & 6.2 & 31800 & 1.55 \\
12.0 & 34550 & 1.39 & 19.7 & 53900 & 1.29 & 11.0 & 43000 & 1.31 \\
20.0 & 55000 & 1.34 & 30.36 & 65600 & 1.27 & 19.4 & 56950 & 1.30 \\
42.5 & 89000 & 1.30 & - & - & - & 28.2 & 69700 & 1.27 \\
- & - & - & - & - & - & 41.3 & 80930 & 1.26 \\
\hline
\end{tabular}

Table 5.10. Number average molecular weight and polydispersity index as a function of monomer conversion for styrene polymerizations in the presence of $0.11 \mathrm{~mol} \%$ styryl-SG1 at $125^{\circ} \mathrm{C}$ and three different pressures.

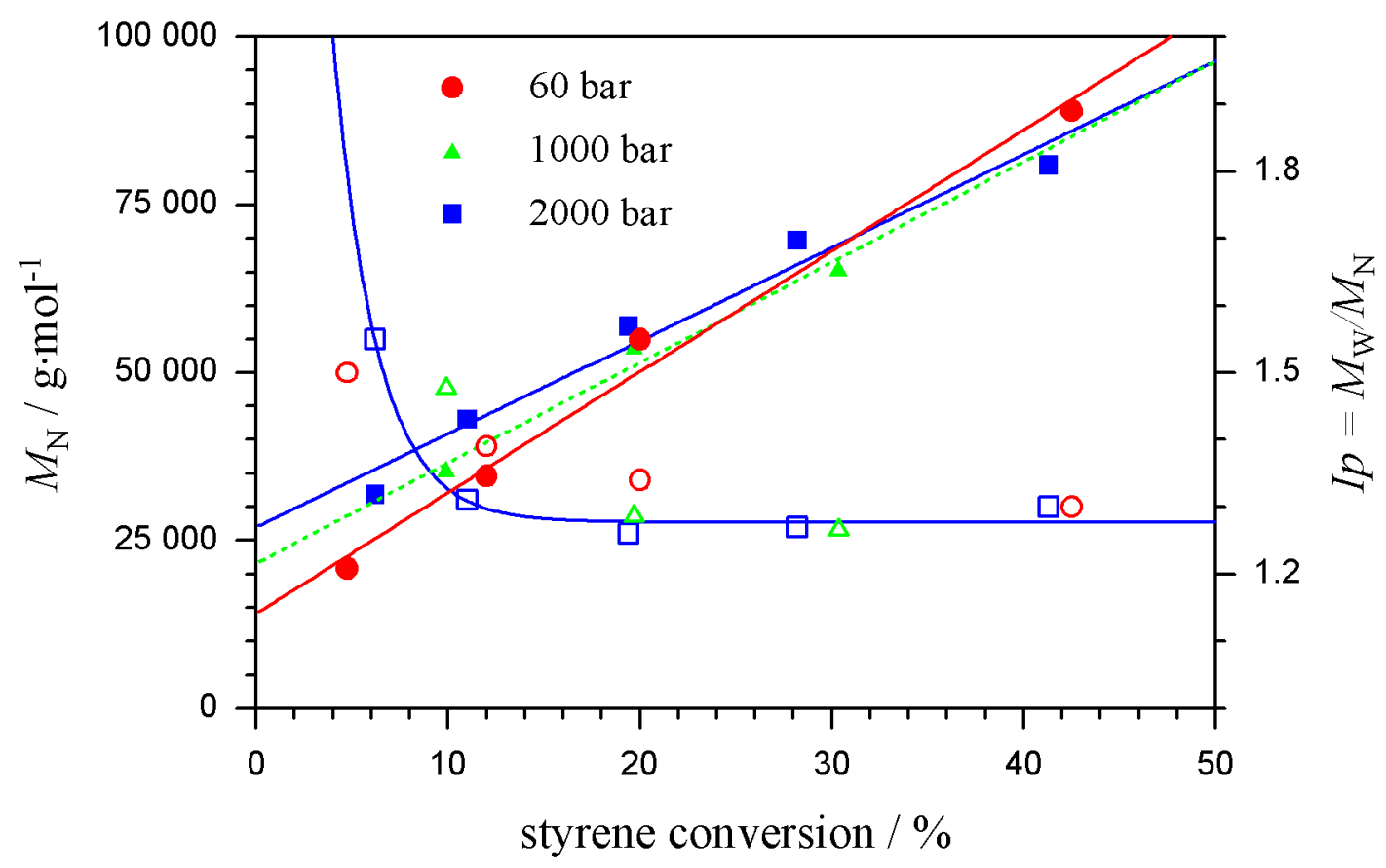

Fig.5.11. Conversion dependence of molecular weight (filled points) and polydispersity index (open points) for styrene polymerizations in the presence of $0.11 \mathrm{~mol} \%$ styryl-SG1 at $125^{\circ} \mathrm{C}$ and pressures between 60 and 2000 bar. 


\subsubsection{Styrene CRP with TEMPO}

Rate data of styrene bulk polymerizations in the presence of TEMPO at $125^{\circ} \mathrm{C}$ and several pressures are shown in Fig. 5.12. Polymerization rate with TEMPO is slower compared to the situation with SG1, which is due to the fact that SG1 is a bulky nitroxide, which dissociates more rapidly than does TEMPO. As has been observed with SG1, pressure clearly increases polymerization rate. The conversion index, $\ln \left([M]_{0} /[M]\right)$, exhibits a slight downward curvature. Experimental curves are fit, by a non linear regression, to a function of the type $\mathrm{a}+\mathrm{b} \cdot t^{\mathrm{c}}$. The values of the exponent $\mathrm{c}$ are close to $2 / 3$ so that the conversion index $\ln \left([M]_{0} /[M]\right)$ seems to follow the rate law deduced by Fischer (Eq. 2.9).

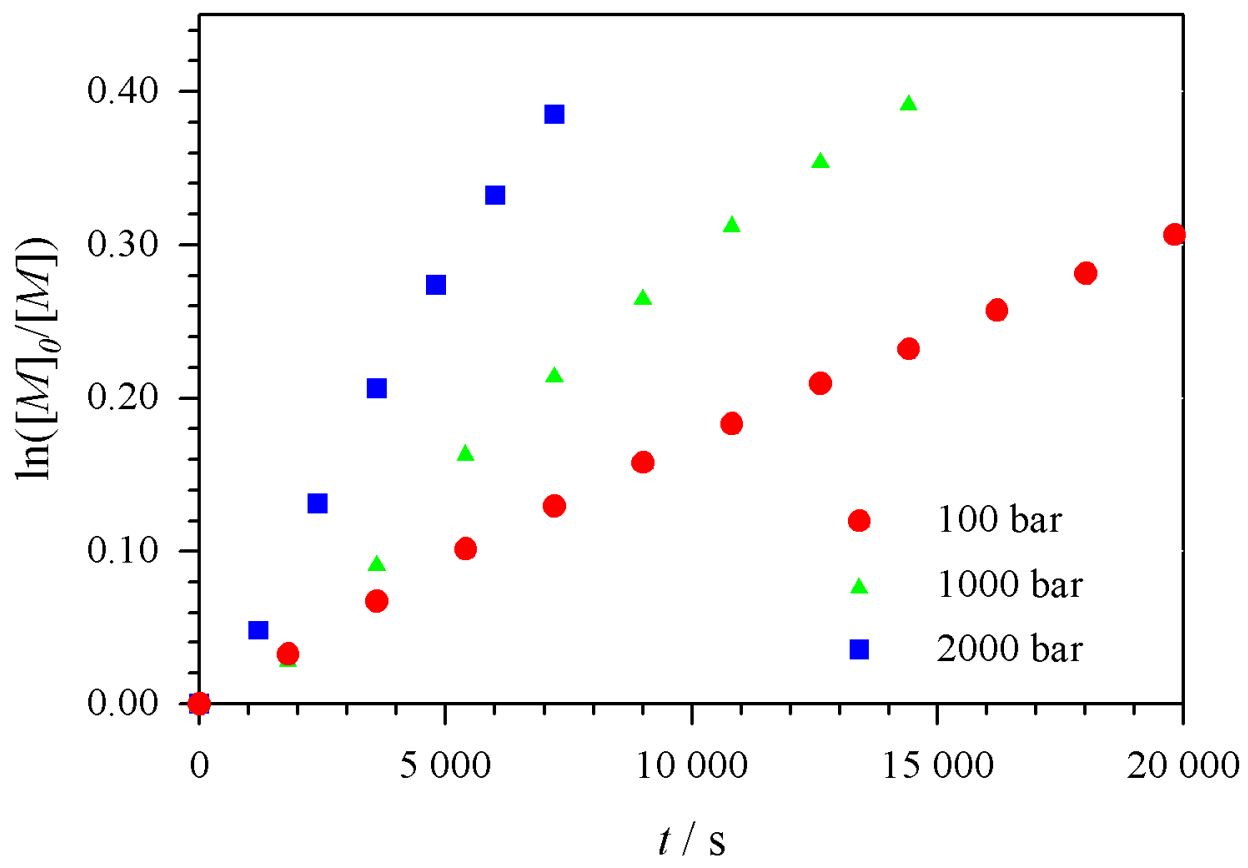

Fig. 5.12. $\ln ([M] d[M])$ plotted vs time for styrene polymerizations in the presence of 0.101 mol\% styryl-TEMPO at $125^{\circ} \mathrm{C}$ and three pressures.

The experimental results for styrene polymerization with styryl-TEMPO are presented in Table 5.13. At $125^{\circ} \mathrm{C}$ and at high alkoxyamine concentration, an effective control of styrene polymerization is observed. The linear molar mass evolution observed at these pressures is very close to the theoretical predictions determined by the ratio $n_{\mathrm{S}} / n_{\mathrm{I}}{ }^{*} M_{\mathrm{S}}$ (38 000 g. $\mathrm{mol}^{-1}$ at $40 \%$ styrene conversion). In Fig. 5.14, extrapolation to zero conversion of the molar mass evolutions reaches 2000,5000 and $11000 \mathrm{~g} \cdot \mathrm{mol}^{-1}$ for the experiments carried 
out at 100, 1000 and 2000 bar, respectively. Low polydispersities are obtained at monomer conversions above $10 \%$ for each pressure.

\begin{tabular}{|c|c|c|c|c|c|c|c|c|}
\hline \multicolumn{3}{|c|}{100 bar } & \multicolumn{3}{|c|}{1000 bar } & \multicolumn{3}{|c|}{2000 bar } \\
\hline $\begin{array}{c}\text { conversion } \\
\%\end{array}$ & $\begin{array}{c}M_{\mathrm{N}} \\
\mathrm{g} \cdot \mathrm{mol}^{-1}\end{array}$ & $I p$ & $\begin{array}{c}\text { conversion } \\
\%\end{array}$ & $\begin{array}{c}M_{\mathrm{N}} \\
\mathrm{g} \cdot \mathrm{mol}^{-1}\end{array}$ & $I p$ & $\begin{array}{c}\text { conversion } \\
\%\end{array}$ & $\begin{array}{c}M_{\mathrm{N}} \\
\mathrm{g} \cdot \mathrm{mol}^{-1}\end{array}$ & $I p$ \\
\hline 5.7 & 7900 & 2.70 & 9.9 & 2.10 & 2.10 & 5.5 & 15400 & 2.20 \\
\hline 7.8 & 7300 & 2.10 & 20.3 & 1.41 & 1.41 & 9.9 & 20660 & 1.95 \\
\hline 16.8 & 21400 & 1.35 & 31.2 & 1.25 & 1.25 & 15.6 & 23000 & 1.40 \\
\hline 31.7 & 35000 & 1.30 & 44.8 & 1.25 & 1.25 & 32.8 & 37700 & 1.30 \\
\hline 39.8 & 38700 & 1.25 & - & - & - & 40.2 & 44000 & 1.30 \\
\hline
\end{tabular}

Table 5.13. Number average molecular weight and polydispersity index as a function of monomer conversion for styrene polymerization in the presence of $0.101 \mathrm{~mol} \%$ styryl-TEMPO at $125^{\circ} \mathrm{C}$ and three pressures.

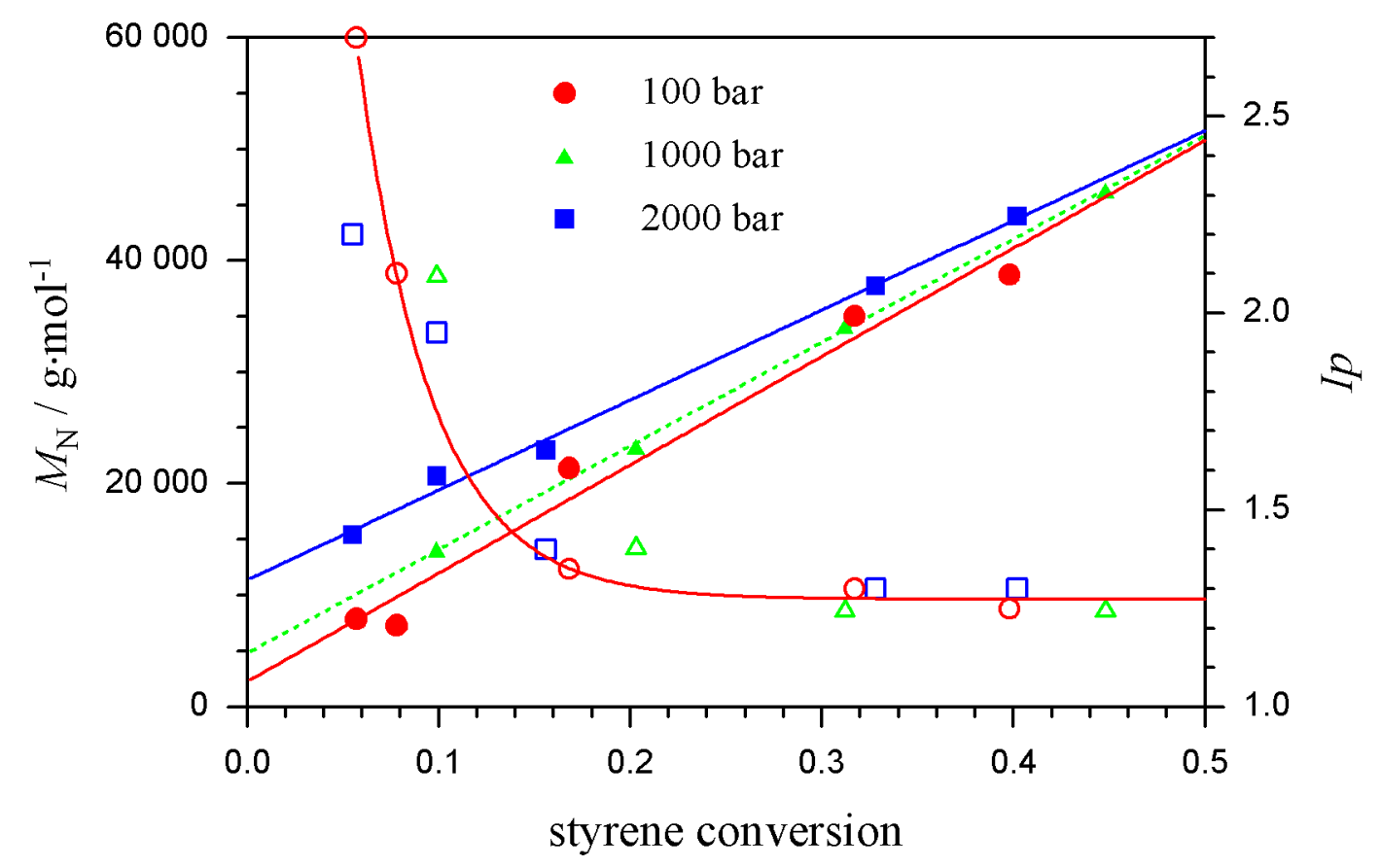

Fig. 5.14. Conversion dependence of molecular weight (filled points) and polydispersity index (open points) for styrene polymerizations in the presence of $0.101 \mathrm{~mol} \%$ styryl-TEMPO at $125^{\circ} \mathrm{C}$ and three pressures. 


\subsubsection{Styrene CRP with DPAIO}

Styrene polymerizations have also been carried out in the presence of $0.10 \mathrm{~mol} \%$ styryl-DPAIO which is a very stable alkoxyamine because of the delocalisation of electrons on the five-membered ring. Fig. 5.15 shows the curved evolution of the conversion index, $\ln \left([M]_{0} /[M]\right)$, with polymerization time. Toward higher pressure, polymerization rate increases in the expected way. The polymerization rate is higher than in case of styrylTEMPO but not so fast than with styryl-SG1, which was not expected because of the low dissociation rate of the alkoxyamine styryl-DPAIO.

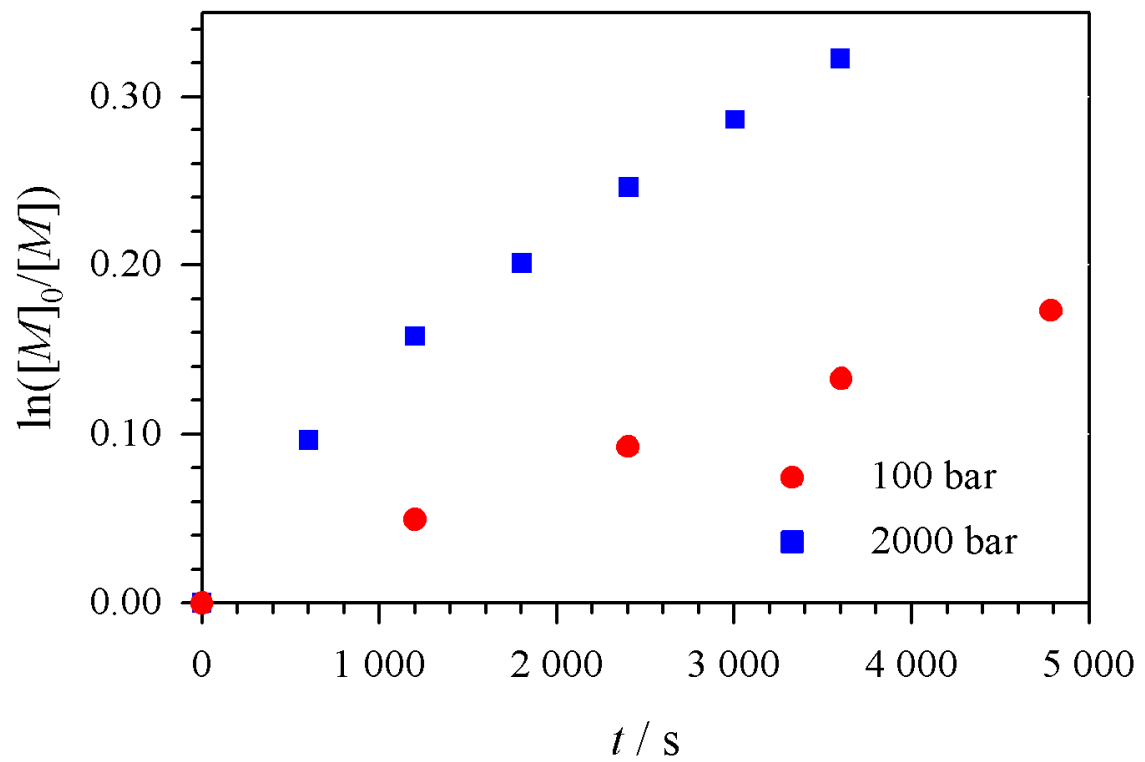

Fig. 5.15. $\ln ([M] d[M])$ vs time plot for styrene polymerizations in the presence of 0.10 mol\% styryl-DPAIO at $125^{\circ} \mathrm{C}$ and two pressures.

Molecular weights and polydispersities obtained for the different experiments are summarized in Table 5.16. Molar masses are independent of monomer conversion and the polydispersities are close to 2, which suggests that no controlled polymerization occurs. Molecular weights are dependent on pressure : $M_{\mathrm{N}}$ obtained at 100 bar is close to 50000 $\mathrm{g} \cdot \mathrm{mol}^{-1}$ whereas $M_{\mathrm{N}}$ is close to $130000 \mathrm{~g} \cdot \mathrm{mol}^{-1}$ at $2000 \mathrm{bar}$, irrespective of the monomer conversion. Molar masses obviously are controlled by propagation, chain-transfer and termination rates. As in the conventional free-radical styrene polymerization, high pressure enhances propagation rate which will be the reason for the observed increase in $M_{\mathrm{N}}$ with polymerization pressure. 


\begin{tabular}{ccc|ccc}
\hline \multicolumn{3}{c|}{$100 \mathrm{bar}$} & \multicolumn{3}{|c}{$2000 \mathrm{bar}$} \\
\hline conversion & $M_{\mathrm{N}}$ & Ip & conversion & $M_{N}$ & $I p$ \\
$\%$ & $\mathrm{~g} \cdot \mathrm{mol}^{-1}$ & & $\%$ & $\mathrm{~g} \cdot \mathrm{mol}^{-1}$ & \\
\hline 5.0 & 39300 & 3.01 & 5.0 & 130000 & 1.90 \\
16.0 & 60900 & 1.80 & 15.0 & 139000 & 1.87 \\
31.0 & 42000 & 1.85 & 30.0 & 132000 & 1.94 \\
46.0 & 35000 & 2.32 & 47.0 & 127000 & 1.93 \\
\hline
\end{tabular}

Table 5.16. Number average molecular weight and polydispersity index as a function of monomer conversion for styrene polymerizations in the presence of $0.10 \mathrm{~mol} \%$ styryl-DPAIO at $125^{\circ} \mathrm{C}$ and two pressures.

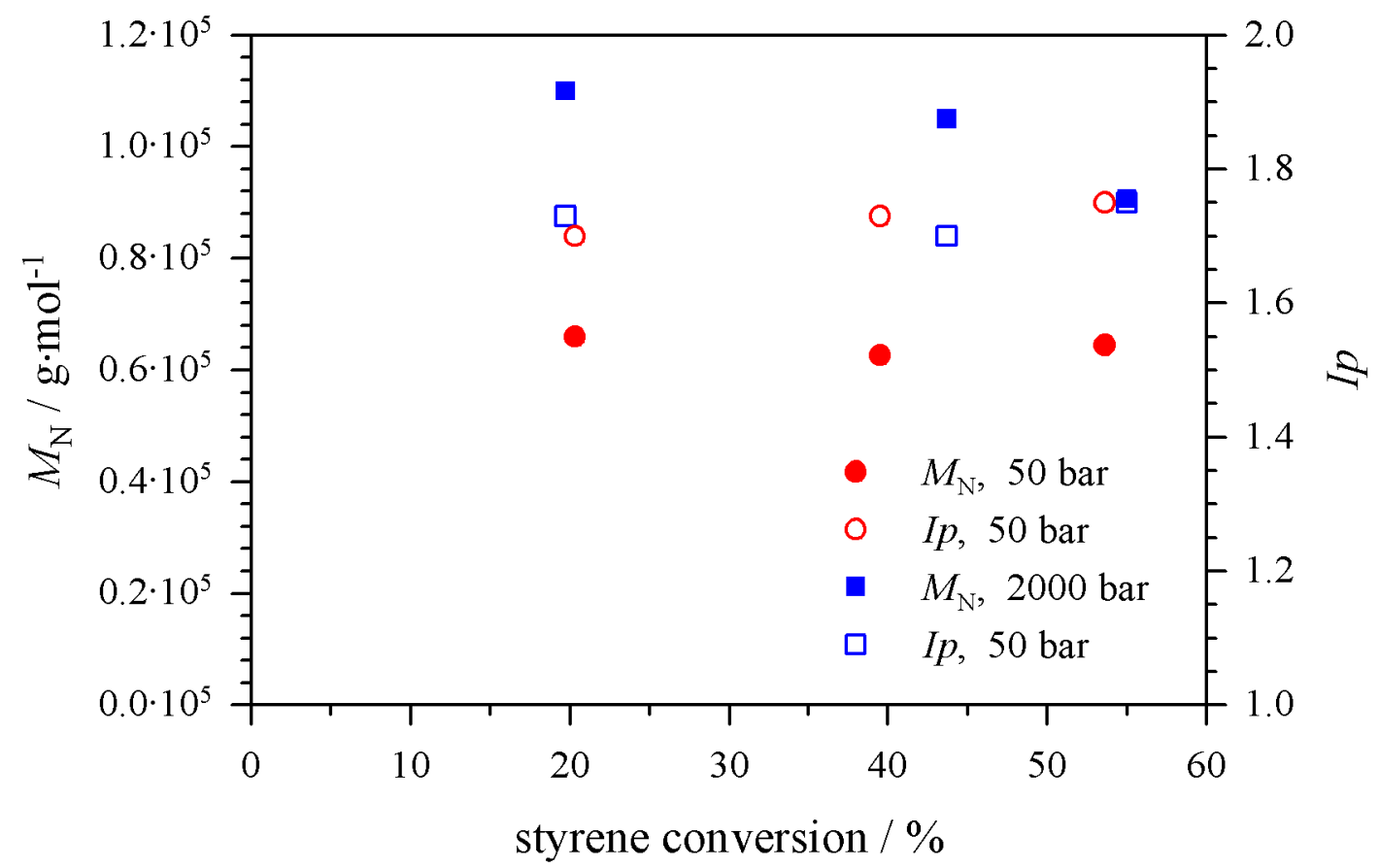

Fig. 5.17. Conversion dependence of molecular weight and polydispersity index for styrene polymerizations in the presence of $0.10 \mathrm{~mol} \%$ styryl-DPAIO at $140^{\circ} \mathrm{C}$ and two pressures, 50 and 2000 bar.

To check whether the observed failure toward controlling radical polymerization is due to a too low dissociation rate of the styryl-DPAIO and then an inadequate equilibrium constant ${ }^{17}$, polymerizations were also carried out at higher temperature. Figure 5.17 shows 
the molecular weights and polydispersity indices measured as a function of styrene conversion for polymerizations at $140^{\circ} \mathrm{C}$. The almost constant molar masses which are observed at different monomer conversions and the polydispersities close to 1.7 demonstrate that styrylDPAIO, even at this high temperature, is not capable of controlling styrene polymerization. The dependence of molar mass on pressure, $M_{\mathrm{N}}=64000 \mathrm{~g} \cdot \mathrm{mol}^{-1}$ at $50 \mathrm{bar}$ and close to 100 $000 \mathrm{~g} \cdot \mathrm{mol}^{-1}$ at $2000 \mathrm{bar}$, shows that DPAIO predominantly acts as a transfer agent.

\section{4 (Meth)acrylate CRP under high pressure}

\subsubsection{Butyl acrylate CRP with SG1}

SG1 has already been reported by Tordo et al. ${ }^{24}$ to be a good mediator for butyl acrylate CRP. Here SG1-mediated butyl acrylate polymerizations were carried out over a wide range of pressures at $125^{\circ} \mathrm{C}$. Fig. 5.18 shows the plot of $\ln \left([M]_{0} /[M]\right)$ versus time at 50 and 2000 bar at $125^{\circ} \mathrm{C}$ for BA polymerizations in the presence of $0.11 \mathrm{~mol} \%$ styryl-SG1.

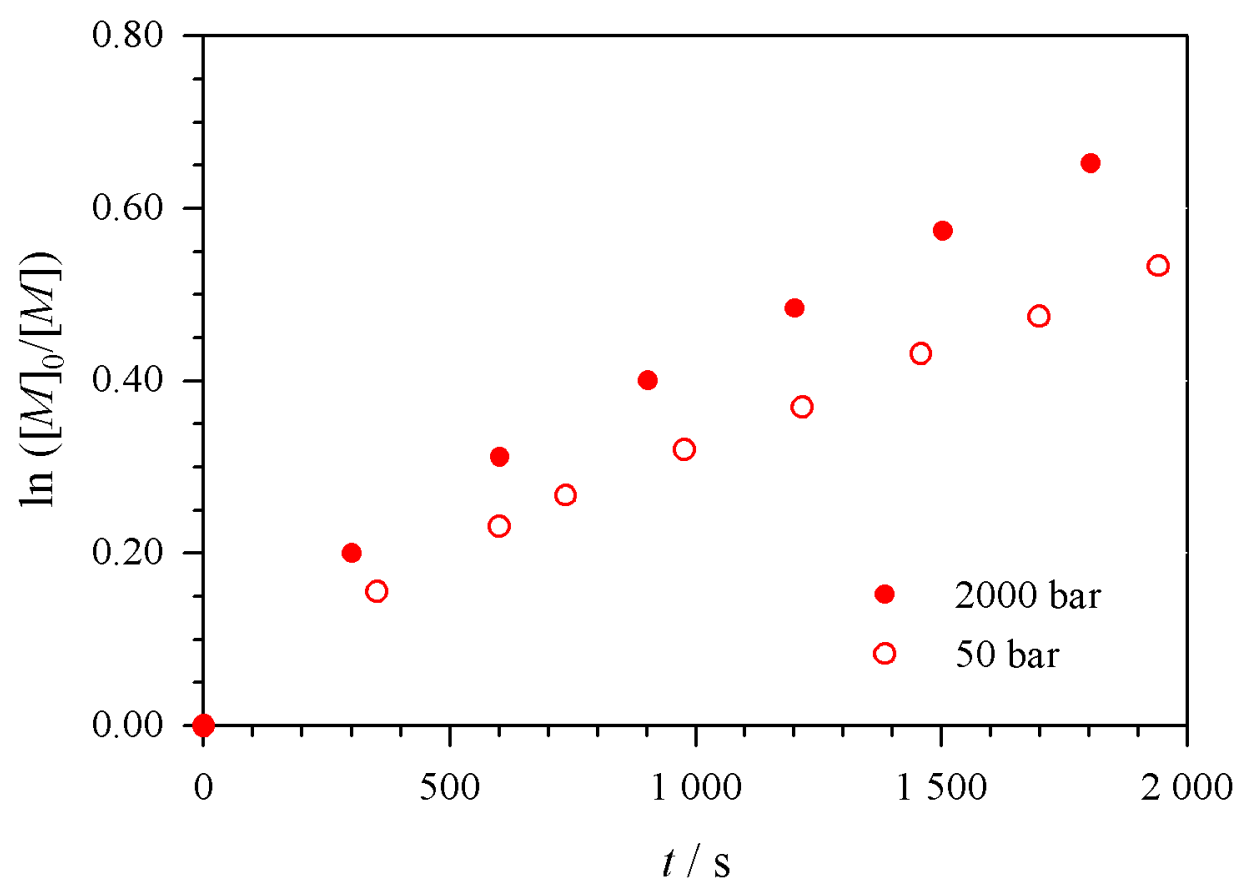

Fig. 5.18. $\quad \ln \left([M]_{d}[M]\right)$ vs time plot for BA polymerizations in the presence of $0.11 \mathrm{~mol} \%$ styryl-SG1 at $125^{\circ} \mathrm{C}$ and at 50 and 2000 bar. 
As was expected from equation 1.1, polymerization is faster at higher pressure. Fig 5.18 shows that the $\ln \left([M]_{0} /[M]\right)$ versus time correlation is not linear but exhibits a downward curvature. Experimental curves are fitted, by non-linear regression, to a function of the type a $+\mathrm{b} \cdot t^{\mathrm{c}}$, where the values of the exponent $\mathrm{c}$ are close to 0.65 for the experiments at 50 and 2000 bar. The non-linearity of the $\ln \left([M]_{0} /[M]\right)$ vs time plot observed in Fig. 5.18 indicates the minor contribution of termination in BA CRP in the presence of SG1. The conversion index $\ln \left([M]_{0} /[M]\right)$ follows the rate law deduced by Fischer (see equation 2.9), where $\mathrm{c}$ is $2 / 3$, that means which the persistent radical effect happens under the influence of termination reaction.

GPC measurements on polymer samples for SG1-mediated BA polymerization carried out up to different degrees of monomer conversion at $125^{\circ} \mathrm{C}$ and at pressures up to $2000 \mathrm{bar}$ yield the number-average molecular weights, $M_{\mathrm{N}}$, and polydispersity indices, $I p$, reported in Table 5.19 and plotted in Fig. 5.20. The linear increase of $M_{\mathrm{N}}$ with monomer conversion and the low polydispersities, around 1.5 at conversions above $10 \%$, demonstrate that SG1 controls styrene polymerization at $125^{\circ} \mathrm{C}$ even under high pressure. The molar mass evolution with conversion does not pass through the origin, probably because of the poorer control at low conversion. Nevertheless, satisfactory polydispersities are obtained up to $40 \%$ conversion, irrespective of the applied pressure.

\begin{tabular}{ccc|ccc}
\hline \multicolumn{3}{c|}{50 bar } & \multicolumn{3}{c}{$2000 \mathrm{bar}$} \\
\hline conversion & $M_{\mathrm{N}}$ & $I p$ & conversion & $M_{\mathrm{N}}$ & $I p$ \\
$\%$ & $\mathrm{~g} \cdot \mathrm{mol}^{-1}$ & & $\mathrm{~g} \cdot \mathrm{mol}^{-1}$ & \\
\hline 10.3 & 29000 & 1.65 & 20.0 & 42300 & 1.60 \\
20.0 & 44500 & 1.60 & 31.0 & 60500 & 1.53 \\
28.3 & 61000 & 1.64 & 42.2 & 81000 & 1.50 \\
38.9 & 65000 & 1.44 & 58.8 & 83200 & 1.50 \\
51.0 & 97000 & 1.37 & - & - & - \\
\hline
\end{tabular}

Table 5.19. Number average molecular weight and polydispersity index as a function of monomer conversion for BA polymerizations in the presence of $0.11 \mathrm{~mol} \%$ styryl-SG1 at $125^{\circ} \mathrm{C}$ and two pressures. 


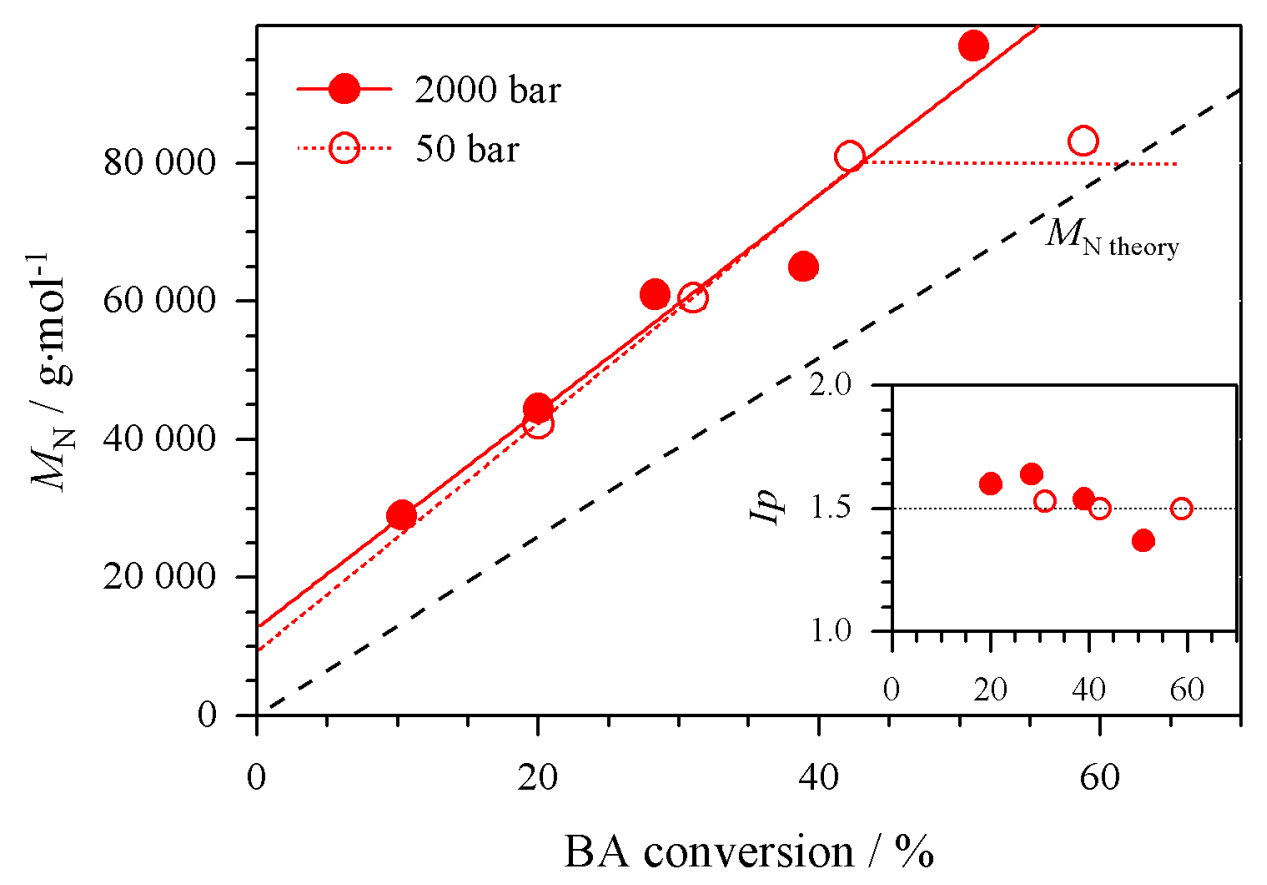

Fig. 5.20. Plot of molecular weight and polydispersity index (insert) as a function of monomer conversion for butyl acrylate polymerizations in the presence of 0.11 mol\% styryl-SG1 at $125^{\circ} \mathrm{C}$ and at 50 (open points) and 2000 bar (filled points).

The plot of molecular weight as a function of monomer conversion for butyl acrylate polymerizations carried out in the presence of 0.11 mol\% styryl-SG1 is reported in Fig. 5.20. Polydispersity vs monomer conversion is plotted in insert. Styryl-SG1-mediated polymerization under high pressure allows the production of high molecular weight material (approximately $10^{5} \mathrm{~g} \cdot \mathrm{mol}^{-1}$ ) with polydispersity smaller than 1.5 . Polymerizations performed at low pressure do not yield such high molar masses. At lower pressure, the number average molecular weight, $M_{\mathrm{N}}$, independent of the alkoxyamine concentration, reaches an upper limiting value of about $80000 \mathrm{~g} \cdot \mathrm{mol}^{-1}$. The MWDs obtained are not so narrow as the ones for SG1-mediated styrene polymerization, especially at high initiator concentration (see Section 5.3.1). Polydispersity indices are slightly lower for high-pressure polymerizations, 1.4 and 1.6 at 2000 and 50 bar, respectively.

\subsubsection{Nitroxide-mediated methyl methacrylate polymerization}

MMA polymerizations were carried out at $95^{\circ} \mathrm{C}$ and 50 and 2000 bar in the presence of approximately $0.1 \mathrm{~mol} \%$ of two alkoxyamine : 
- styryl-SG1 (S-SG1); reaction temperature around $60^{\circ} \mathrm{C}$

- cyano, isopropyl-DPAIO (CN-DPAIO); reaction temperature around $60^{\circ} \mathrm{C}$

The kinetic data is compared with results from AIBN-initiated MMA polymerization. The plots of conversion index vs time for alkoxyamine-mediated MMA polymerizations are reported in Fig. 5.21. In the MMA polymerizations high conversion were reached. The highest conversion was obtained in the high-pressure polymerization with styryl-SG1. At 1 bar and otherwise the same conditions, most nitroxides do not allow for reaction to conversions higher than $20 \%{ }^{18-19}$. AIBN-initiated MMA polymerization at $95^{\circ} \mathrm{C}$ yields high conversion with a fast polymerization rate. The plot of the conversion index vs time is quite linear, which indicates a low termination rate, at both pressures applied.

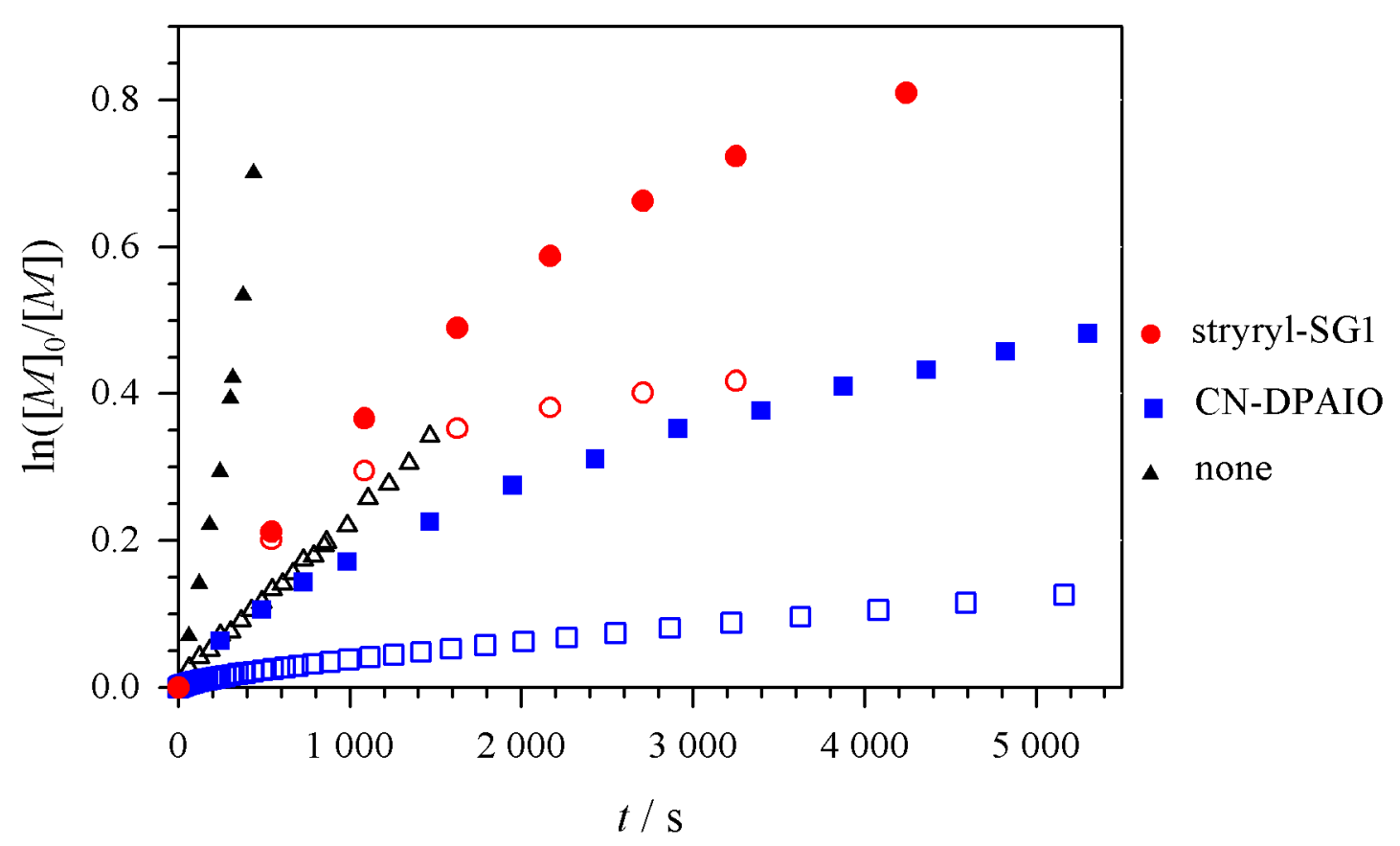

Fig. 5.21. $\ln ([M] d[M])$ vs time plot for methyl methacrylate polymerizations in the presence of various alkoxyamines at $95^{\circ} \mathrm{C}$ and at 50 (open symbols) and 2000 bar (filled symbols) and without alkoxyamine. The reaction conditions are summarized in Table 5.22.

In the presence of an alkoxyamine, the polymerization rate is much lower than in case of conventional MMA FRP. As expected, polymerization rates are higher in the presence of SG1 than in the presence of DPAIO due to the high reactivity of the open-chain nitroxide. High pressure induces a higher polymerization rate because of the increase of $k_{\mathrm{p}}$ and decrease 
of $k_{\mathrm{t}}$. The high obtained conversion, especially under high pressure, indicates that the propagating radicals are not that rapidly converted to an unreactive hydroxylamine. The combination of high pressure with a very reactive nitroxide (SG1) or with a five-memberedring nitroxide (DPAIO) may have a positive influence on the control of methyl methacrylate polymerization. Molecular weight and polydispersity as a function of monomer conversion for alkoxyamine-mediated MMA polymerization at $95^{\circ} \mathrm{C}$ and at pressures up to 2000 bar are summarized in Table 5.22.

\begin{tabular}{|c|c|c|c|c|}
\hline alkoxyamine & $P /$ bar & $M_{\mathrm{N}} / \mathrm{g} \cdot \mathrm{mol}^{-1}$ & $I p=M_{\mathrm{W}} / M_{\mathrm{N}}$ & $\begin{array}{c}\text { MMA conversion } \\
\%\end{array}$ \\
\hline S-SG1* & 50 & 129000 & 2.10 & 22.2 \\
\hline S-SG1* & 50 & 115000 & 1.78 & 28.5 \\
\hline S-SG1* & 50 & 119000 & 1.86 & 34.9 \\
\hline S-SG1* & 2000 & 248000 & 2.05 & 55.8 \\
\hline S-SG1* & 2000 & 213000 & 2.09 & 35.0 \\
\hline S-SG1* & 2000 & 220000 & 2.08 & 20.3 \\
\hline CN-DPAIO $* *$ & 50 & 23000 & 2.06 & 25.0 \\
\hline CN-DPAIO $* *$ & 50 & 35000 & 2.30 & 8.2 \\
\hline CN-DPAIO $* *$ & 50 & 27500 & 2.20 & 16.5 \\
\hline CN-DPAIO $* *$ & 2000 & 120000 & 2.56 & 38.7 \\
\hline $\mathrm{CN}-\mathrm{DPAIO} * *$ & 2000 & 147000 & 2.21 & 24.5 \\
\hline $\mathrm{CN}-\mathrm{DPAIO} * *$ & 2000 & 164000 & 2.89 & 13.0 \\
\hline None*** & 50 & 77000 & 1.83 & 29.3 \\
\hline None*** & 2000 & 130000 & 2.36 & 50.5 \\
\hline
\end{tabular}

Table 5.22. Number average molecular weight and polydispersity as a function of monomer conversion for methyl methacrylate polymerizations $\left(95^{\circ} \mathrm{C}\right.$, various pressures, bulk polymerization) in the presence of various alkoxyamines. 
Weight average molecular weight, $M_{\mathrm{W}}$, as a function of MMA conversion for alkoxyamine-mediated polymerizations at $95^{\circ} \mathrm{C}$ and at pressures up to 2000 bar is given in Fig. 5.23. Experimental polydispersities are close to 2 and higher, irrespective of the experimental conditions, for the systems under investigation. Molecular weights are independent of monomer conversion, which demonstrates that there is no control. Molecular weights seem to be determined by the experimental conditions, in particular by the applied pressure and the nature of the alkoxyamine. In fact, high pressure induces much higher molecular weights for conventional MMA FRP and also for polymerization in the presence of SG1 and DPAIO. The use of styryl-SG1 leads to much higher molecular weights than in conventional FRP with AIBN as the initiator. On the other hand, the use of CN-DPAIO leads to much lower molecular weights.

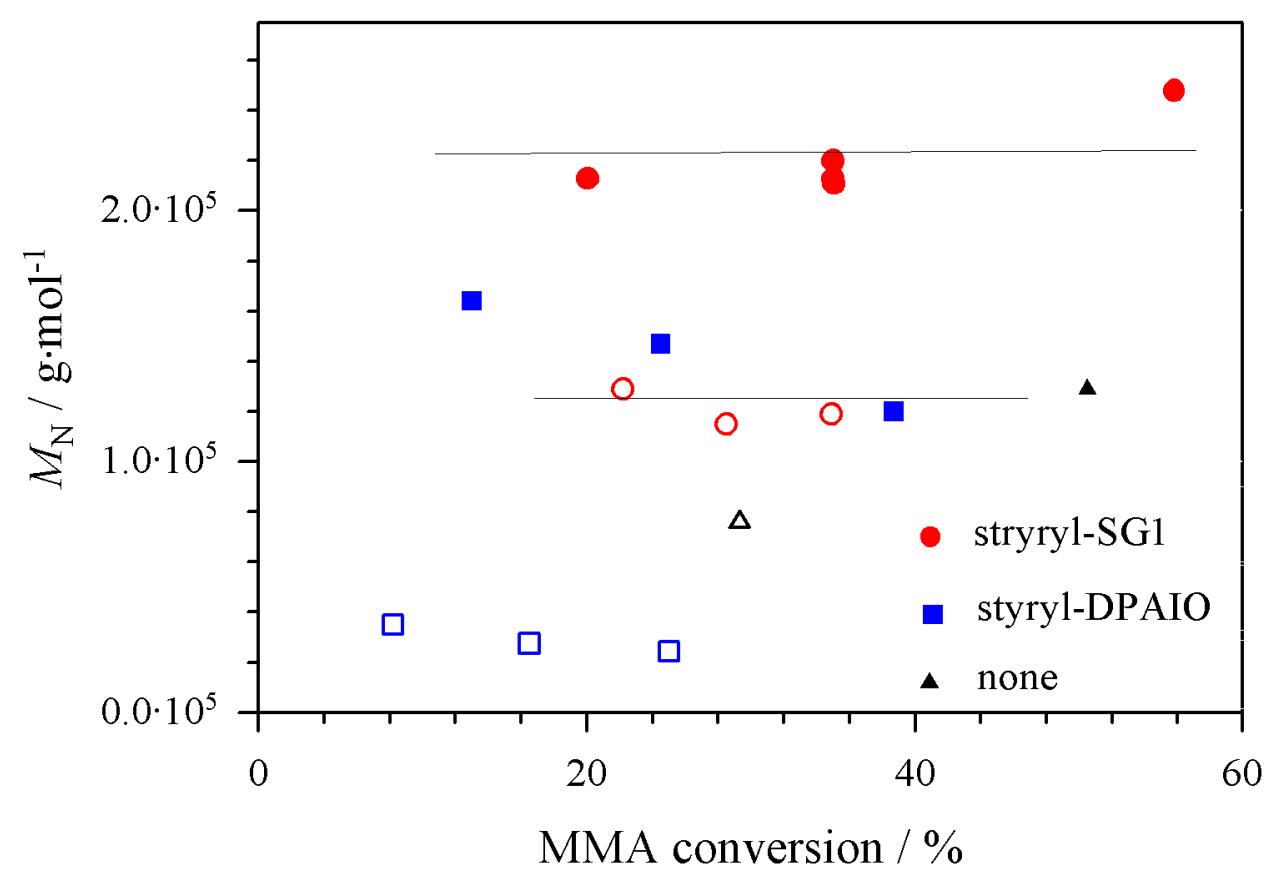

Fig. 5.23. Plot of molecular weight as a function of monomer conversion for methyl methacrylate polymerizations in the presence of two alkoxyamines, at $95^{\circ} \mathrm{C}$ and at 50 (open points) and 2000 bar (filled points) and without alkoxyamine. Reaction conditions are summarized in Table 5.22. 


\subsection{Nitroxide-mediated ethene polymerization under high pressure}

\subsubsection{Bulky nitroxides : DTBN, SG1}

Ethene polymerizations have been carried out in the presence of bulky nitroxides, such as DTBN and SG1. Styrene CRP with DTBN and SG1 are characterized by high polymerization rates due to high dissociation rates of dormant species compared with styrylTEMPO. Ethene homopolymerizations in the presence of DTBN were spectroscopically investigated over the temperature range from 140 up to $180^{\circ} \mathrm{C}$ at 2000 bar. Polymerizations have been performed in the presence of an excess of nitroxide as compared to the initiator $([\mathrm{DTBN}] /[\mathrm{DTBP}]=2.5)$. Experimental initiator concentrations are defined as molar fractions $[\mathrm{I}] /[\mathrm{E}]$ where $[\mathrm{I}]$ and $[\mathrm{E}]$ refer to initiator and ethene molar concentrations, respectively. Low fractions are expressed in molar ppm.

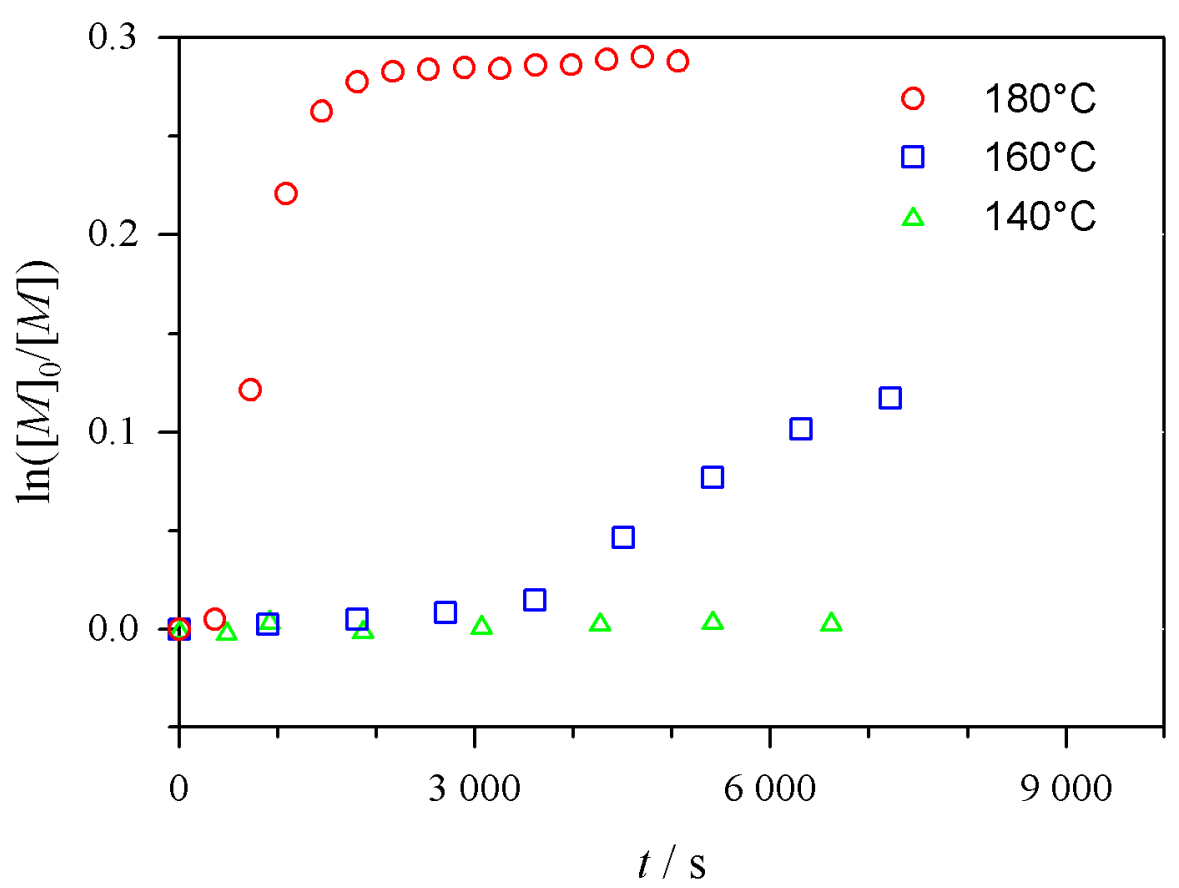

Fig. 5.24. $\ln ([M] d[M])$ vs time plot for ethene polymerization in the presence of $50 \mathrm{ppm}$ DTBP at 2000 bar and three temperatures. $[D T B N] /[D T B P]=2.5$.

In Figure 5.24 is shown the plot of the conversion index $\ln \left([M]_{0} /[M]\right)$ versus reaction time for DTBN-mediated ethene polymerization at 2000 bar and three temperatures. At $140^{\circ} \mathrm{C}$, DTBN-mediated ethene polymerization is inhibited or retarded for more than two hours. This inhibition time is shorter at higher temperature, 60 and $10 \mathrm{~min}$ at 160 and $180^{\circ} \mathrm{C}$, 
respectively. The polymerization rate following the retardation period is much faster at high temperature and $7 \%$ conversion are reached and $27 \%$ conversion in $2000 \mathrm{~s}$ at 160 and $180^{\circ} \mathrm{C}$, respectively. The observed $\ln \left([M]_{0} /[M]\right)$ vs time plots are not linear, and at $180^{\circ} \mathrm{C}$ the typical behavior of conventional free-radical polymerization is seen. At $180^{\circ} \mathrm{C}$, an upper limiting value of the conversion index is observed after $2000 \mathrm{~s}$ polymerization time.

Further ethene polymerizations have been carried out at different nitroxide concentrations at $160^{\circ} \mathrm{C}$ and 2000 bar in order to investigate the influence of nitroxide concentration. The duration of the inhibition period, as reported in Table 5.25, increases with the ratio [nitroxide]/[initiator], extending over more than 2 hours for a ratio [DTBN]/[DTBP] $=9$ at $160^{\circ} \mathrm{C}$.

$[\mathrm{DTBN}] /[\mathrm{DTBP}]=2.5 \quad[\mathrm{DTBN}] /[\mathrm{DTBP}]=4.5 \quad[\mathrm{DTBN}] /[\mathrm{DTBP}]=9.0$

duration of inhibition

period

$60 \min$

$75 \min$

$>2$ hours

Table. 5.25. Inhibition period in ethene polymerizations in the presence of 50 ppm DTBP at $160^{\circ} \mathrm{C}$ and at 2000 bar for different ratios [DTBN]/[DTBP].

Ethene polymerizations in the presence of SG1 were carried out with an alkoxyamine styryl-SG1 (S-SG1) as initiator to avoid the recombination of radicals issued from the initiator decomposition and to eliminate the inhibition period observed with DTBN-mediated styrene polymerization. Polymerization rates and molecular weights have been studied at several alkoxyamine concentrations. The conversion index vs time plot for S-SG1-mediated ethene polymerization performed at $190^{\circ} \mathrm{C}$ and 2000 bar are reported in Fig. 5.26. The ethene polymerization rate at low S-SG1 concentration $([\mathrm{S}-\mathrm{SG} 1] /[$ ethene] $=53 \mathrm{ppm})$ is quite small compared to the experiments at higher alkoxyamine concentration. The conversion index exhibits a downward curvature and reaches $13 \%$ in one hour. Polymerization rates at high alkoxyamine concentration (107 and 200 ppm) are higher and are similar. $13 \%$ conversion are reached after $15 \mathrm{~min}$. The polymerization rates seem to be dependent on the alkoxyamine concentration up to a limiting upper concentration which is between 50 and $100 \mathrm{ppm}$. This dependence of polymerization rate on alkoxyamine concentration is not consistent with the 
mechanism of CRP as proposed by Fukuda (see Section 2.4), who assumed that the polymerization rate is independent of the S-DTBN concentration for styrene polymerization.

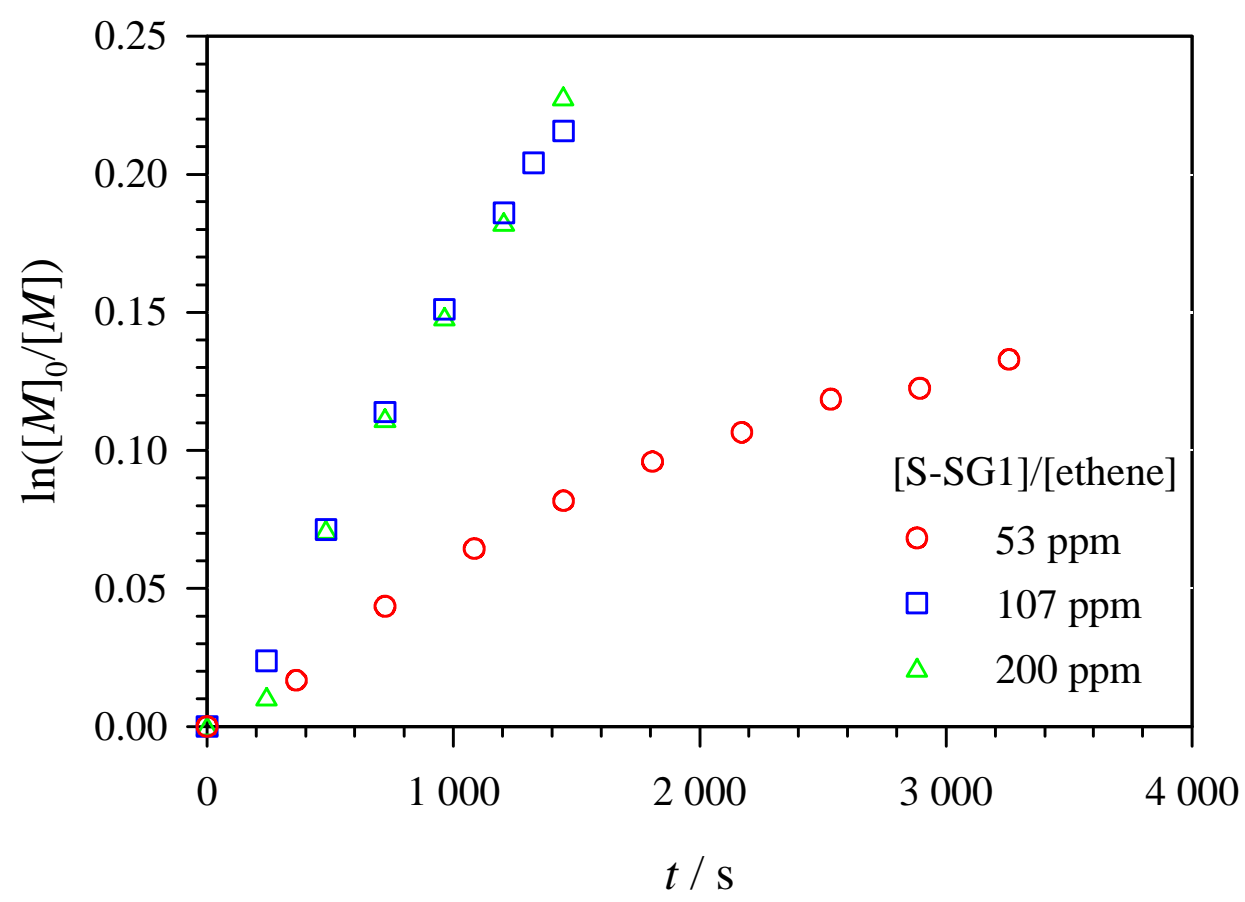

Fig. 5.26. $\ln ([\mathrm{M}] d /[M])$ vs time plot for ethene polymerizations at $190^{\circ} \mathrm{C}$ and 2000 bar in the presence of three concentrations of S-SG1.

The SEC analysis allowed for the determination of the polymer MWDs. The plot of the molar mass as a function of monomer conversion is presented in Fig. 5.27. Low S-SG1 concentration induces high weight average molecular weights close to $M_{\mathrm{W}}=110000 \mathrm{~g} \cdot \mathrm{mol}^{-1}$, whereas at high alkoxyamine concentrations, 107 and $200 \mathrm{ppm}, M_{\mathrm{Ws}}$ close to $70000 \mathrm{~g} \cdot \mathrm{mol}^{-1}$ are obtained. Both experiments at 107 and 200 ppm S-SG1 also show similar polymerization rates. Polydispersity indices observed are close to 3 . Irrespective of the experimental conditions, molar masses do not linearly increase with monomer conversion and polydispersity indices are not typical of a CRP. 


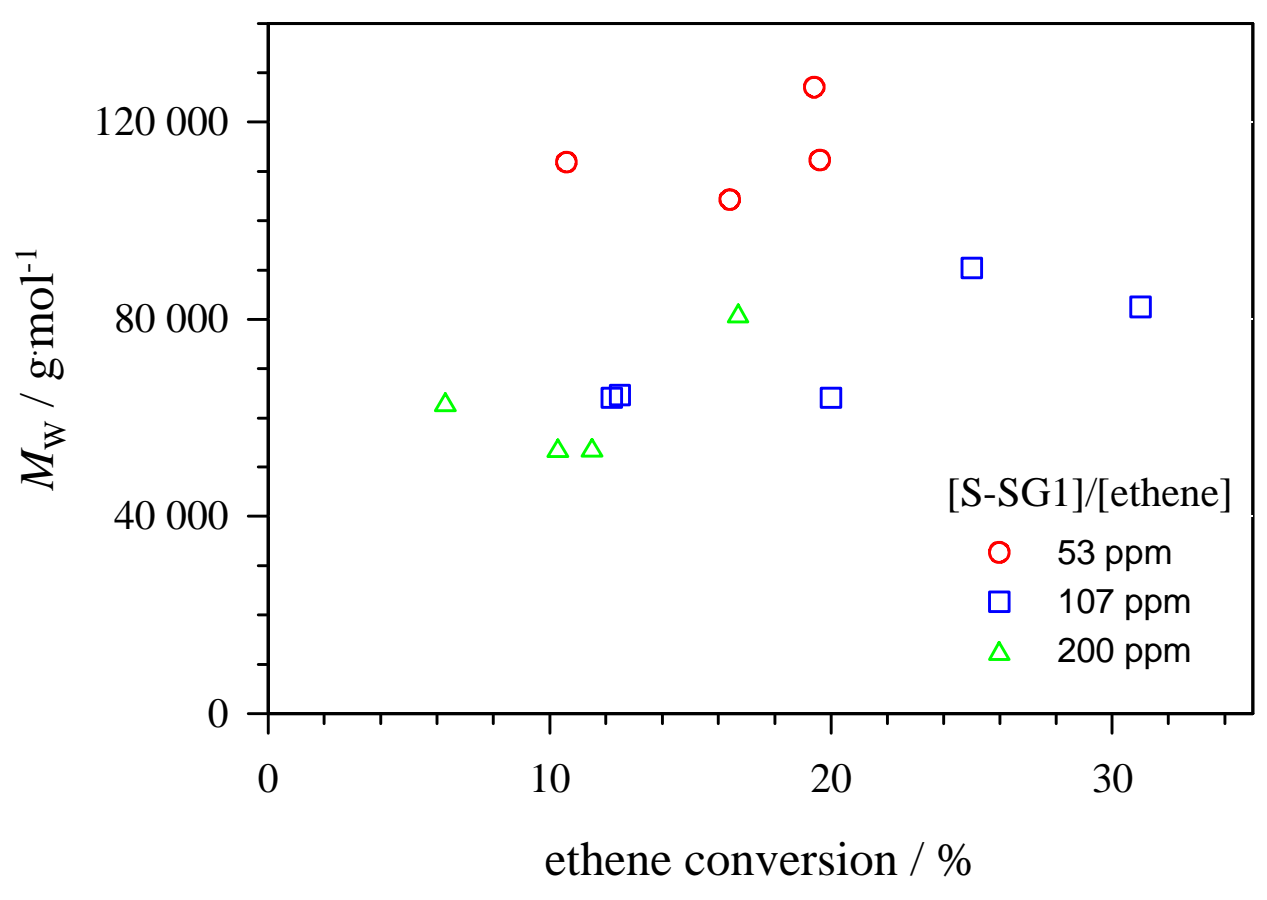

Fig. 5.27. Weight average molecular weight as a function of monomer conversion for ethene polymerizations at $190^{\circ} \mathrm{C}$ and 2000 bar in the presence of three concentrations of $S-S G 1$.

\subsubsection{Commercially available nitroxides : TEMPO and TEMPO-derivatives}

TEMPO and TEMPO-derived substances were the most common nitroxides used to control the styrene polymerization before several newly designed nitroxides have been developed. Controlled ethene polymerizations were also reported in the presence of TEMPO and its derivatives ${ }^{20}$. In this work, ethene polymerizations with TEMPO in combination with DTBP, or with an alkoxyamine, e.g. hexyl-TEMPO, were investigated over a wide range of temperatures. Polymerization rates and molecular weights have been studied from 180 up to $220^{\circ} \mathrm{C}$ at 2000 bar and at different ratios [TEMPO]/[DTBP], from 1 up to 10 . 


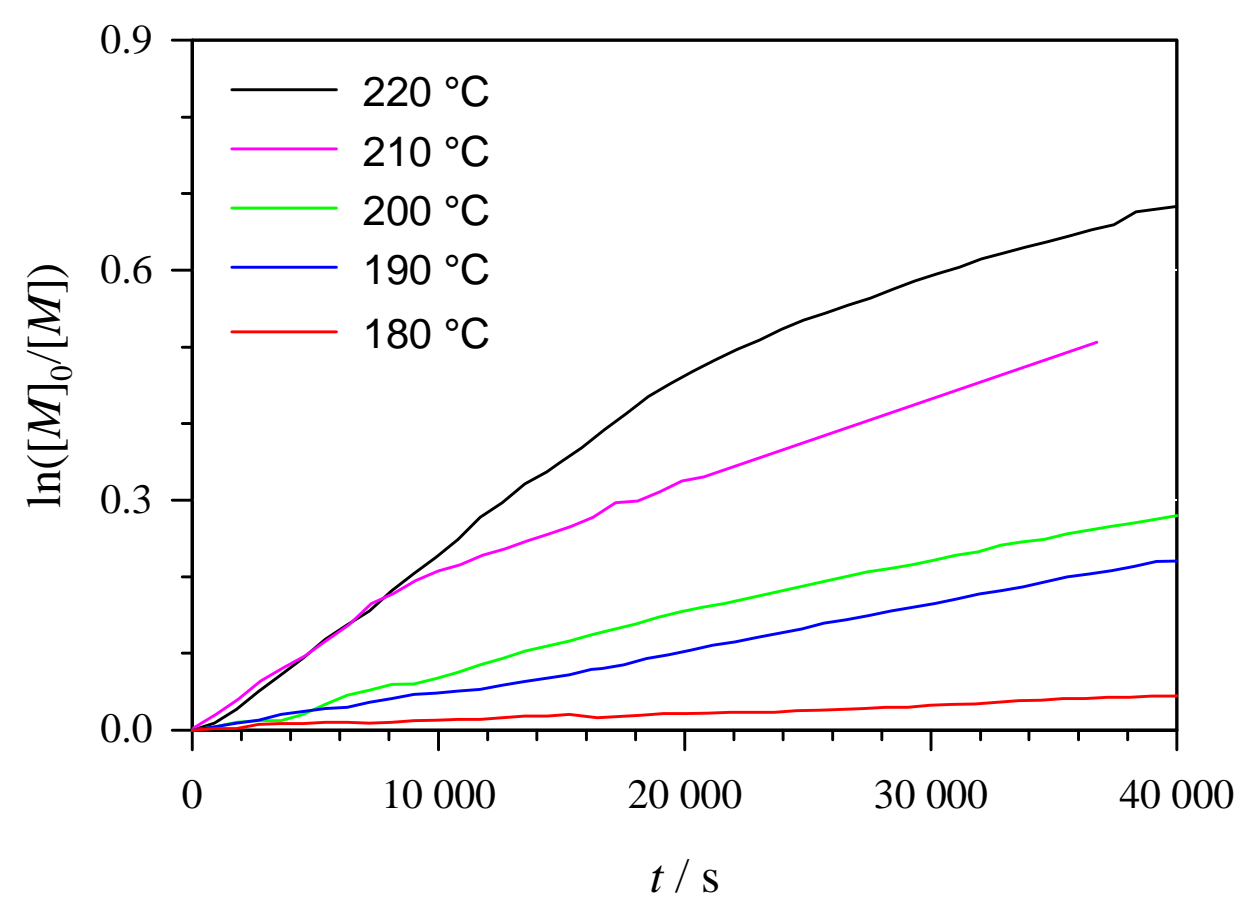

Fig. 5.28. $\ln ([M] d[M])$ vs time plot for TEMPO-mediated ethene polymerizations at 2000 bar and at different temperatures in the presence of 50 ppm DTBP. $[T E M P O] /[D T B P]=2$.

The plot of the conversion index vs polymerization time for ethene polymerization $\left([\right.$ TEMPO]/[DTBP] $=2)$ at 2000 bar and between 180 and $220^{\circ} \mathrm{C}$ is shown in Fig. 5.28. The DTBP efficiency is assumed to be lower than 1. Thus there is an excess of the persistent radical over the growing radicals from the beginning of the polymerization. The rate of ethene polymerization in the presence of TEMPO is very low and linear at $180^{\circ} \mathrm{C}(10 \%$ conversion in 20 hours) and reaches $70 \%$ conversion in 12 hours at $220^{\circ} \mathrm{C}$. Under these conditions, the $\ln \left([M]_{0} /[M]\right)$ vs time plot exhibits a slight downward curvature, indicating that termination occurs.

The influence of the ratio [TEMPO]/[DTBP] on the conversion index vs time plot is described in Fig. 5.29. Ethene polymerizations were performed at $200^{\circ} \mathrm{C}$ and 2000 bar for a ratio $\mathrm{R}=[\mathrm{TEMPO}] /[\mathrm{DTBP}]$ between 0 and 10 . In the absence of TEMPO, $\mathrm{R}=0$, the polymerization starts very fast and reaches a limiting upper conversion around $25 \%$ after few minutes. Thereafter a very small conversion due to spontaneous ethene polymerization may be detected. Increasing the TEMPO concentration in the system leads to a decrease of the concentration of free radicals produced by initiator decomposition and the extend of polymerization reached in the first stage is reduced. At $\mathrm{R}=2$, no inhibition is observed and 
the $\ln \left([M]_{0} /[M]\right)$ vs time correlation exhibits a slight downward curvature due to termination. In the presence of an excess of TEMPO, an inhibition period is observed, the duration of which depends on the TEMPO concentration. As in DTBN-mediated ethene polymerization, the inhibition period increases with the size of $\mathrm{R}$. The rate of polymerization is lower at higher ratio of [TEMPO]/[DTBP], probably slowed down by high persistent radical concentrations.

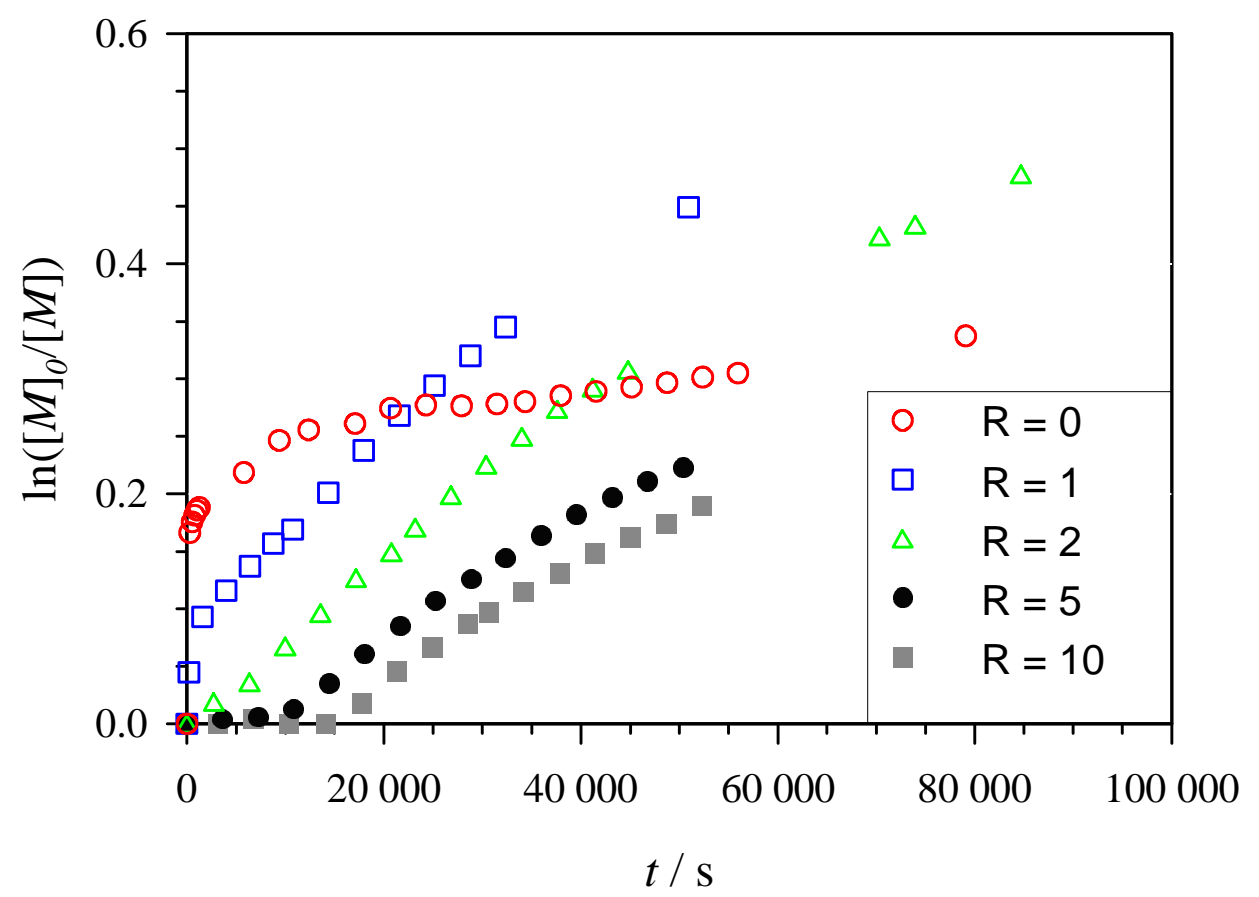

Fig. 5.29. $\ln ([\mathrm{M}] d[\mathrm{M}])$ vs time plot for ethene polymerizations at $200^{\circ} \mathrm{C}$ and $2000 \mathrm{bar}$ for different ratios [TEMPO]/[DTBP]. [DTBP]/[styrene] $=50 \mathrm{ppm}$.

The molar mass and the polydispersity index as a function of monomer conversion for the TEMPO-mediated polymerization at $200^{\circ} \mathrm{C}$ and 2000 bar and at a ratio $[$ TEMPO $] /[\mathrm{DTBP}]=2$ are plotted in Fig. 5.30. Polymerization rate for this experiment has been reported in Fig. 5.28. Very low and conversion-independent number average molecular weights, $M_{\mathrm{N}}$ around $15000 \mathrm{~g} \cdot \mathrm{mol}^{-1}$, are observed. $M_{\mathrm{N}}=56000 \mathrm{~g} \cdot \mathrm{mol}^{-1}$ was expected for $20 \%$ ethene conversion. The polydispersity index increases with monomer conversion from 4 up to 8 at 14 and $50 \%$ ethene conversion, respectively, that is far off the theoretical $I p$ which should be below 1.5. Polyethylene samples, obtained at different ratios $\mathrm{R}$ and at several $T$ and $P$ conditions, all show the same characteristics, i.e. low conversion-independent $M_{\mathrm{N}}$ and high polydispersity indices. All experimental data are reported in Appendix A2. 


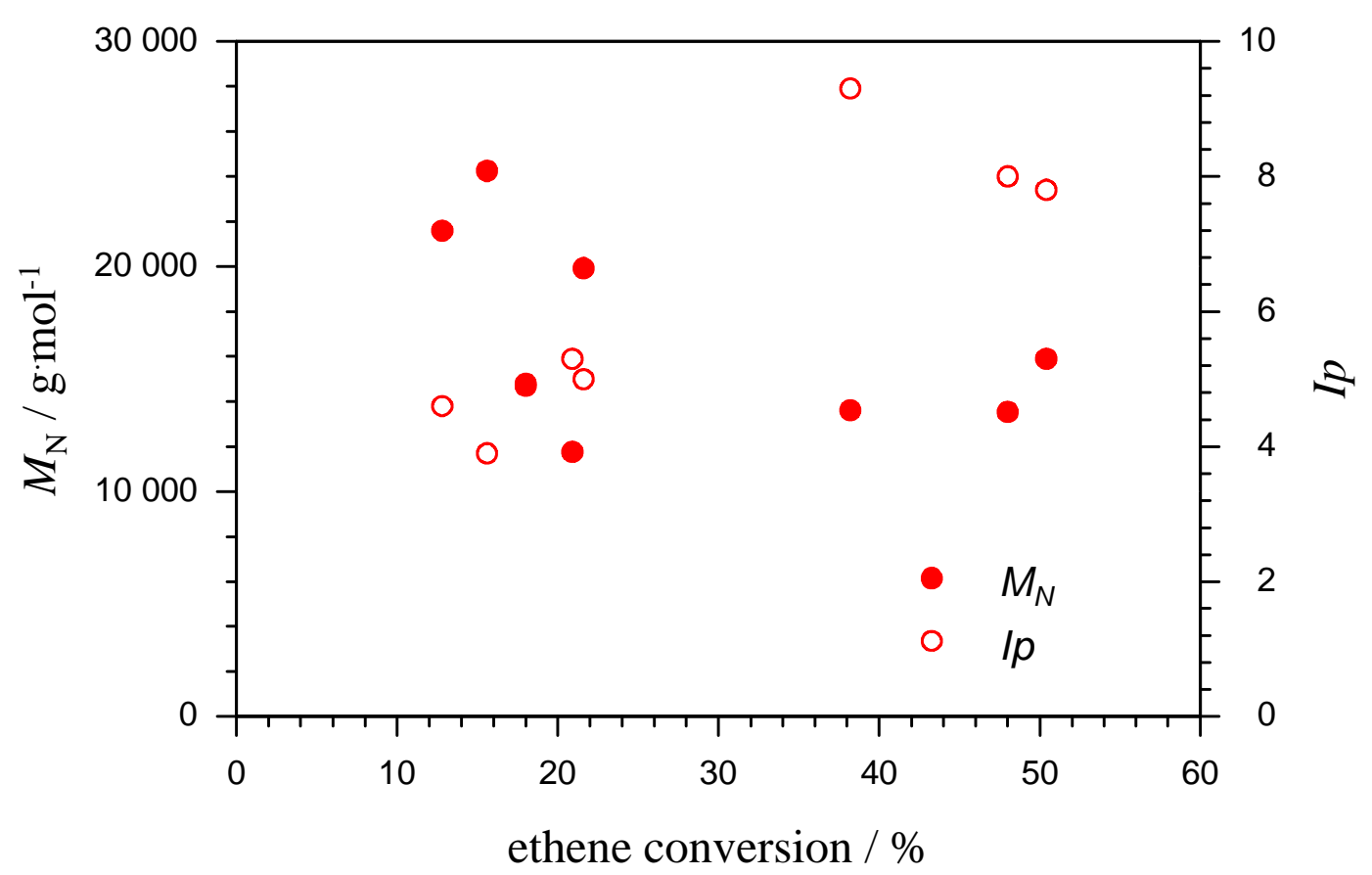

Fig. 5.30. Number average molecular weight and polydispersity index as a function of monomer conversion for ethene polymerizations at $200^{\circ} \mathrm{C}$ and 2000 bar in the presence of $50 \mathrm{ppm}$ DTBP. [TEMPO]/[DTBP] $=2$.

Further ethene polymerizations were carried out in the presence of DTBP and CXA, a TEMPO-derived nitroxide with two nitroxyl radical functions and with a better thermal stability than the one of TEMPO. Polymerization rates observed are similar to TEMPOmediated ethene polymerization under the same conditions. Conversion, molar masses and polydispersities are reported in Table 5.31 for the ethene polymerization in the presence of $\mathrm{CXA}([\mathrm{CXA}] /[\mathrm{DTBP}]=1.1)$ at $220^{\circ} \mathrm{C}$ and 2000 bar.

\begin{tabular}{cccc}
\hline$t / \mathrm{s}$ & conversion $/ \%$ & $M_{\mathrm{N}} / \mathrm{g} \cdot \mathrm{mol}^{-1}$ & $I p$ \\
\hline 3600 & 3.3 & 28000 & 5.3 \\
7200 & 8.7 & 23200 & 6.7 \\
10800 & 14.3 & 24800 & 7.2 \\
\hline
\end{tabular}

Table 5.31. Number average molecular weight and polydispersity index as a function of monomer conversion for CXA-mediated ethene polymerizations at $220^{\circ} \mathrm{C}$ and 2000 bar in the presence of $50 \mathrm{ppm}$ DTBP. [CXA]/[DTBP] $=1.1$. 
The presented results do not show any characteristics of controlled radical polymerization, probably because of a too low stability of the nitroxides used and of a too high stability of the dormant species.

Recent studies ${ }^{21}$ showed that oxygen-centered radicals (such as the ones produced by DTBP decomposition) may induce decomposition of certain nitroxides such as TEMPO. To avoid such reactions, polymerization experiments have been performed using a TEMPOderived alkoxyamine (hexyl-TEMPO) which has a good thermal stability as was checked by $\mathrm{ESR}^{3}$.

In the presence of hexyl-TEMPO, the reproducibility of the conversion vs time correlations of ethene polymerization is very good between 190 and $250^{\circ} \mathrm{C}$. Characteristics of ethene polymerizations performed in the presence of $100 \mathrm{ppm}$ hexyl-TEMPO at $2000 \mathrm{bar}$ and between 190 and $250^{\circ} \mathrm{C}$ are reported in Table 5.32 .

\begin{tabular}{|c|c|c|c|c|c|c|c|c|c|c|}
\hline \multicolumn{4}{|c|}{$210^{\circ} \mathrm{C}$} & \multicolumn{3}{|c|}{$230^{\circ} \mathrm{C}$} & \multicolumn{4}{|c|}{$250^{\circ} \mathrm{C}$} \\
\hline $\begin{array}{l}t \\
\mathrm{~s}\end{array}$ & $\%$ & $\begin{array}{c}M_{\mathrm{W}} \\
\mathrm{g} \cdot \mathrm{mol}^{-1}\end{array}$ & $I p$ & $\%$ & $\begin{array}{c}M_{\mathrm{W}} \\
\mathrm{g} \cdot \mathrm{mol}^{-1}\end{array}$ & $I p$ & $\begin{array}{l}t \\
\mathrm{~s}\end{array}$ & $\%$ & $\begin{array}{c}M_{\mathrm{W}} \\
\mathrm{g} \cdot \mathrm{mol}^{-1}\end{array}$ & $I p$ \\
\hline 3600 & 4.4 & 69500 & 6.1 & 34.7 & 109000 & 4.8 & 800 & 28.1 & 72000 & 7.4 \\
\hline 7200 & 9.2 & 80500 & 5.4 & 53.2 & 274000 & 11.3 & 1800 & 39.8 & 74000 & 10.7 \\
\hline 10800 & 14.0 & 103700 & 6.2 & 64.0 & 134000 & 10.1 & 3300 & 48.9 & 107000 & 14.5 \\
\hline
\end{tabular}

Tab. 5.32. Number average molecular weight and polydispersity index as a function of monomer conversion for ethene polymerizations in the presence of $100 \mathrm{ppm}$ hexyl-TEMPO at 2000 bar and temperatures between $210^{\circ} \mathrm{C}$ and $250^{\circ} \mathrm{C}$.

$\ln \left([M]_{0} /[M]\right)$ at different temperatures increases with time and exhibits a slight downward curvature at temperatures above $230^{\circ} \mathrm{C}$. No inhibition period has been observed. The spontaneous ethene polymerization was also monitored at 2000 bar and several temperatures for comparison. Polymerization rates observed are approximately twofold higher than the spontaneous ethene polymerization (Fig. 5.33). Nevertheless, molecular weights do not linearly increase with monomer conversion and are far from the theoretical molecular weights estimated from the initiator concentration. Polydispersities are very high, typically between 6 and 14 at high conversion. 


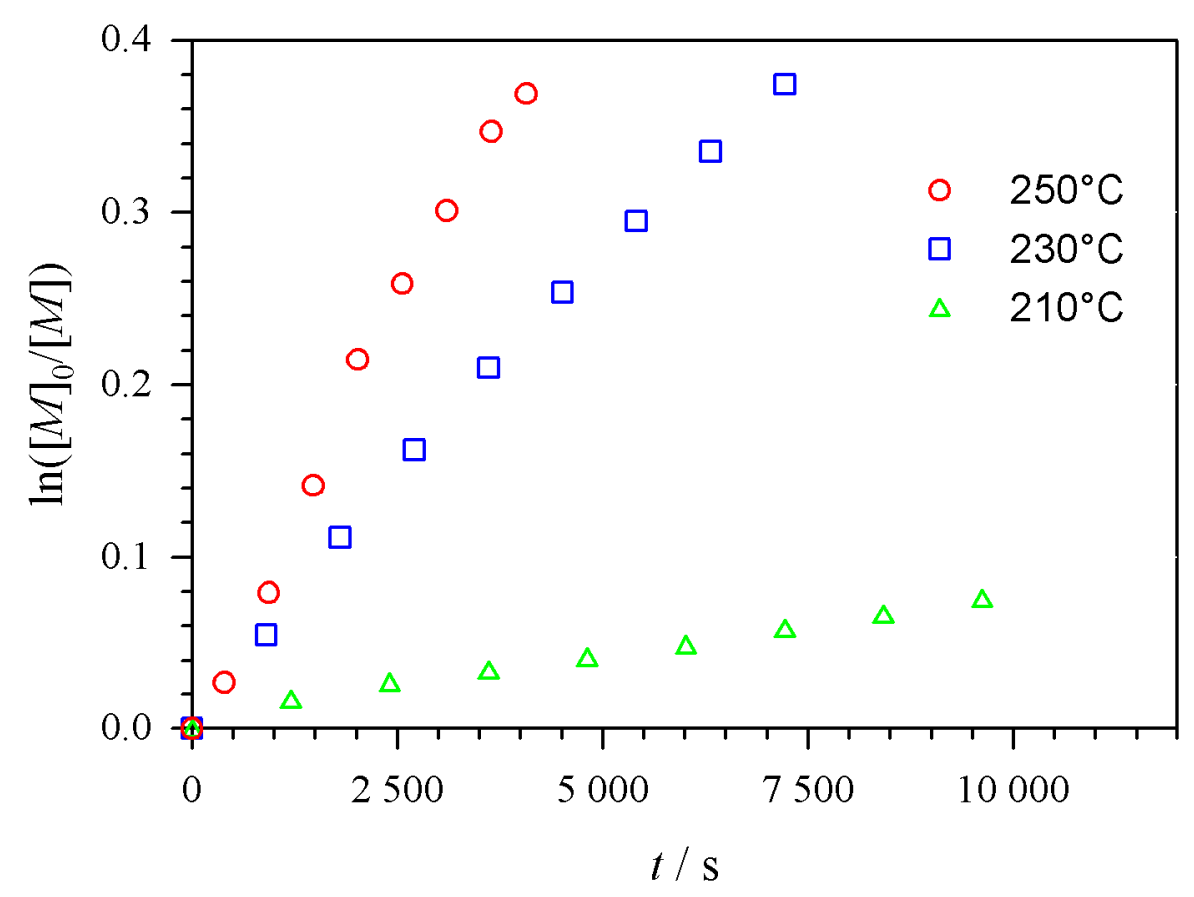

Fig. 5.33. $\ln ([M] d[M])$ vs time plot for spontaneous thermal ethene polymerizations at 2000 bar and three temperatures.

Fig. 5.33 shows that the conversion index vs time plot for spontaneous thermal ethene polymerization is quite linear because of the low and constant initiation rate for spontaneous ethene polymerization. Polymerization rates are much lower than the ones observed in nitroxide-mediated ethene polymerization, so that it is assumed that dormant species or the nitroxide will undergo a bond dissociation and will enhance polymerization rate.

\subsubsection{Thermal nitroxide decomposition}

The thermal nitroxide decomposition has been studied by chromatographic analysis of nitroxide concentration in $n$-heptane solution at 2000 bar and several temperatures. Hexadecane was used as internal standard for the chromatographic analysis. The decomposition of DTBN, TEMPO and hexyl-TEMPO were investigated. The rate coefficients obtained are reported in Table 5.34. The decomposition follows first-order kinetics. The first order reaction of nitroxide degradation is described by the integrated Equation 4.6.

The variation of the first order rate coefficient with temperature can be described by the Arrhenius equation (Eq. 4.7). The activation energy of the decomposition reaction is given by the slope of a plot $\ln k$ versus inverse temperature. 


\begin{tabular}{cc|cc|cc}
\hline \multicolumn{2}{c|}{ DTBN } & \multicolumn{2}{c|}{ TEMPO } & \multicolumn{2}{c}{ hexyl-TEMPO } \\
\hline$T /{ }^{\circ} \mathrm{C}$ & $k / \mathrm{s}^{-1}$ & $T /{ }^{\circ} \mathrm{C}$ & $k / \mathrm{s}^{-1}$ & $T /{ }^{\circ} \mathrm{C}$ & $\mathrm{k} / \mathrm{s}^{-1}$ \\
\hline 120 & $1.7 \cdot 10^{-5}$ & 160 & $1.1 \cdot 10^{-5}$ & 210 & $9.0 \cdot 10^{-6}$ \\
130 & $1.3 \cdot 10^{-4}$ & 180 & $4.6 \cdot 10^{-5}$ & 230 & $2.7 \cdot 10^{-5}$ \\
140 & $5.2 \cdot 10^{-4}$ & 200 & $1.9 \cdot 10^{-4}$ & 250 & $1.8 \cdot 10^{-4}$ \\
150 & $6.3 \cdot 10^{-4}$ & - & - & - & - \\
\hline \multicolumn{2}{c|}{$E_{\mathrm{a}=142 \mathrm{~kJ} \cdot \mathrm{mol}^{-1} *}$} & \multicolumn{2}{c|}{$E_{\mathrm{a}}=162 \mathrm{~kJ} \cdot \mathrm{mol}^{-1} *$} & \multicolumn{2}{c}{$E_{\mathrm{a}}=182 \mathrm{~kJ} \cdot \mathrm{mol}^{-1} *$} \\
\hline
\end{tabular}

$* \mathrm{~A}=2.5 \cdot 10^{14}$

Table 5.34. Rate coefficients of nitroxide decomposition investigated at 2000 bar.

Rate coefficients for the different nitroxides and alkoxyamine investigated are plotted in Figure 5.35.

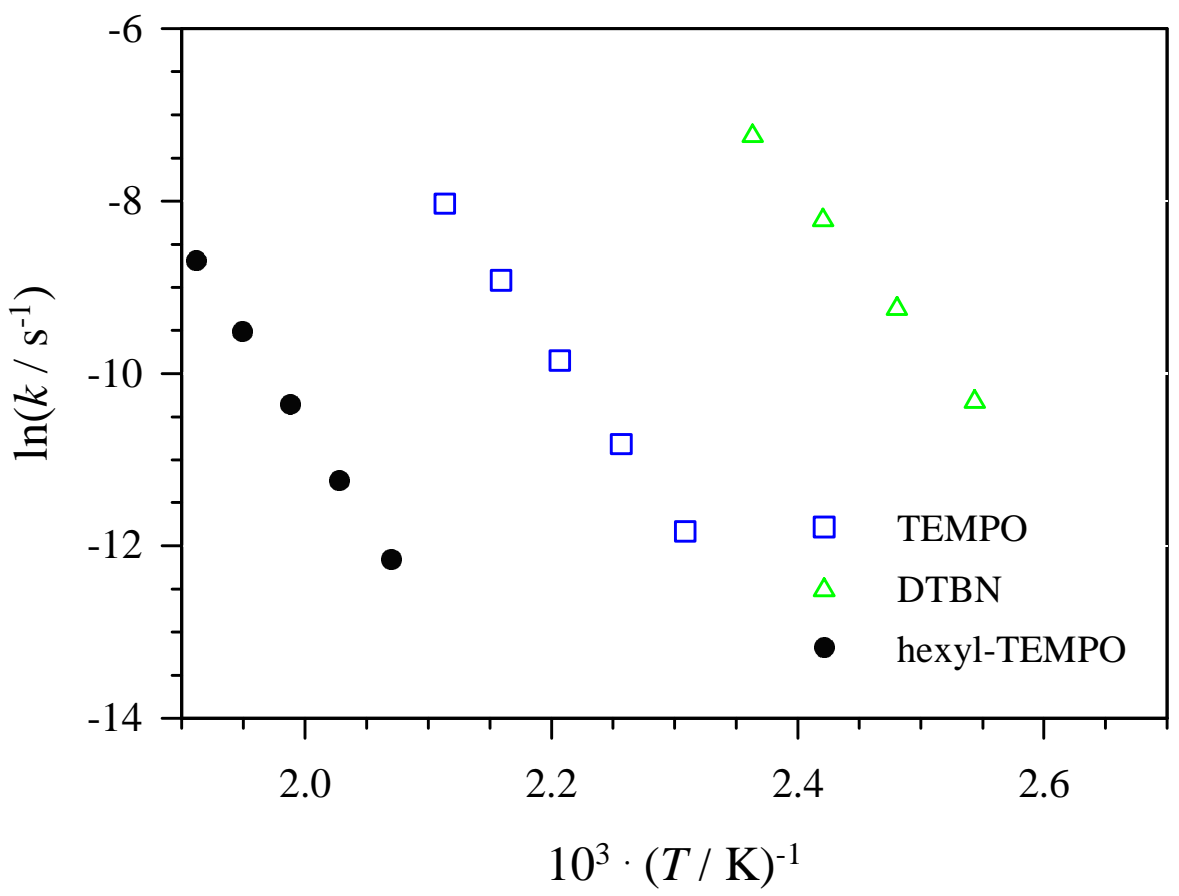

Fig. 5.35. Plot of rate coefficients vs inverse temperature for nitroxide decompositions at 2000 bar. 


\subsubsection{Newly developed nitroxides : DPAIO and derivatives}

Within a joint research project new nitroxides have been developed by Greci (University of Ancona) and Tordo (University of Marseille). The intention was to provide thermally stable but also reactive compounds. A study of DPAIO thermal stability has already been reported ${ }^{22}$. By monitoring the nitroxide concentration via Electron Paramagnetic Resonance spectroscopy (EPR), the nitroxide DPAIO was shown to be stable for two hours at $200^{\circ} \mathrm{C}$ in benzene.

Ethene polymerization has been investigated in the presence of $100 \mathrm{ppm}$ of the alkoxyamine hexyl-DPAIO at 2000 bar and between 150 and $170^{\circ} \mathrm{C}$. In Fig. 5.36 $\ln \left([M]_{0} /[M]\right)$ is plotted vs time for hexyl-DPAIO-mediated ethene polymerizations at 2000 bar and at different temperatures. As compared with the situation in hexyl-TEMPO-mediated polymerization, high polymerization rates are observed even at low temperature : $10 \%$ conversion are reached in $30 \mathrm{~min}$ at $150^{\circ} \mathrm{C}$. The plot of $\ln \left([M]_{0} /[M]\right)$ vs time is almost linear at $150^{\circ} \mathrm{C}$ and exhibits a slight downward curvature at higher temperature.

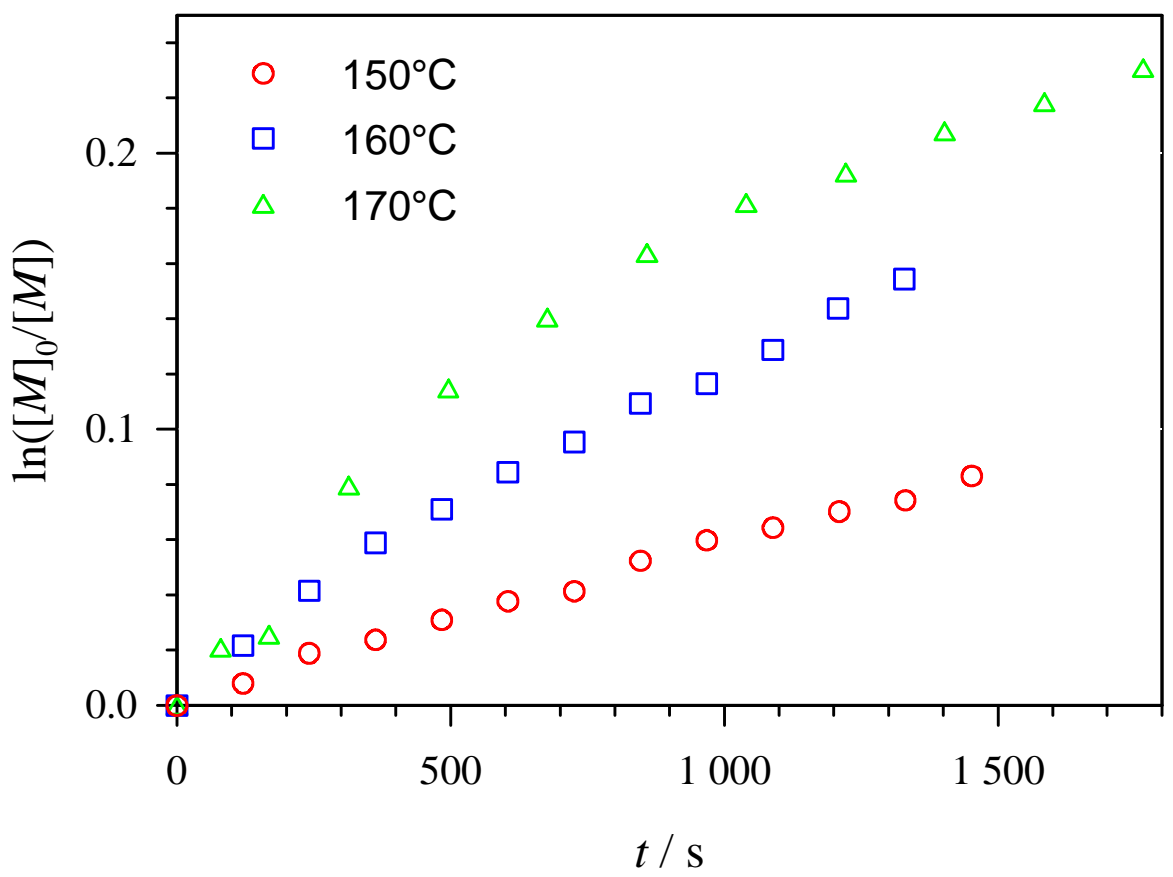

Fig. 5.36. $\ln \left([M]_{d}[M]\right)$ vs time plot for ethene polymerizations at 2000 bar and at different temperatures in the presence of 100 ppm hexyl-DPAIO. 
The thermal stability of the nitroxide DPAIO has been proven by carrying out an ethene polymerization in the presence of the nitroxide at $160^{\circ} \mathrm{C}$ and 2000 bar (without initiator). No polymerization has been observed. The spontaneous thermal ethene polymerization is negligible under these conditions.

The influence of hexyl-DPAIO concentration on polymerization rate and molecular weight was investigated by performing experiments at $160^{\circ} \mathrm{C}$ and 2000 bar over a wide range of alkoxyamine concentrations, from $12 \mathrm{ppm}$ up to $400 \mathrm{ppm}$. The plot of $\ln \left([M]_{0} /[M]\right)$ vs time for hexyl-DPAIO-mediated ethene polymerization at alkoxyamine concentration from 12 up to $400 \mathrm{ppm}$ is shown in Fig. 5.37. Polymerization rates seems to be independent of alkoxyamine concentration between 50 and $400 \mathrm{ppm}$. The experiments at alkoxyamine concentrations of 200 and 400 ppm are slightly shifted due to a short inhibition period at the very beginning of the polymerization. At very low alkoxyamine concentrations $(<50 \mathrm{ppm})$, polymerization rate increases with hexyl-DPAIO concentration, but is independent of alkoxyamine concentration above $100 \mathrm{ppm}$.

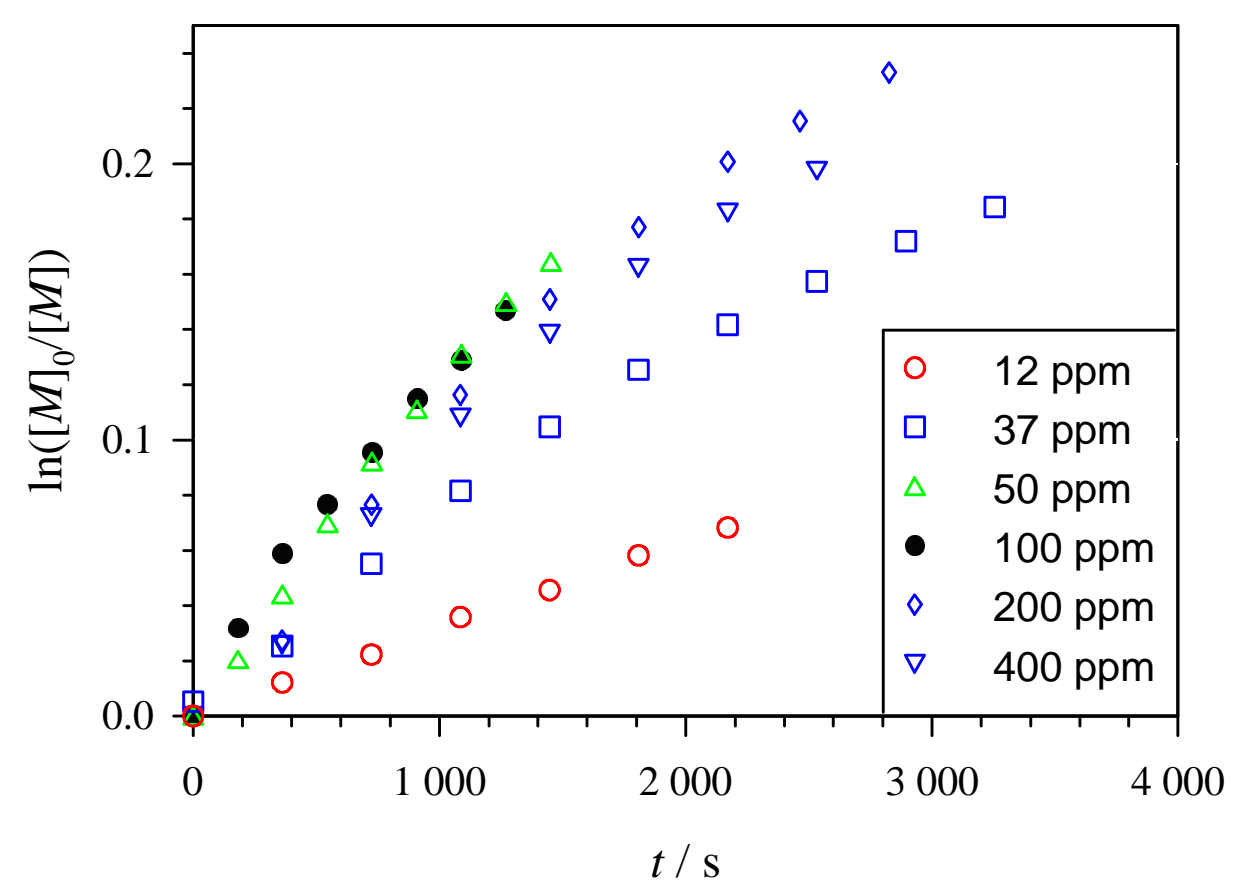

Fig. 5.37. $\ln ([\mathrm{M}] d /[\mathrm{M}])$ vs time plot for ethene polymerizations at $160^{\circ} \mathrm{C}$ and 2000 bar in the presence of different concentrations of hexyl-DPAIO. 
Molecular weights of the peak maximum for hexyl-DPAIO-mediated ethene polymerization at $160^{\circ} \mathrm{C}$ and 2000 bar at different alkoxyamine concentrations are reported in Fig. 5.38. As expected, the alkoxyamine concentration influences molecular weight. Lowering the concentration leads to high molecular weights. A limiting molar mass of about 60000 $\mathrm{g} \cdot \mathrm{mol}^{-1}$ seems to be reached at high alkoxyamine concentration. At 200 and $400 \mathrm{ppm}$ of hexyl-DPAIO, the molecular weights observed are close to $50000 \mathrm{~g} \cdot \mathrm{mol}^{-1}$, independent of monomer conversion. At low concentration $(50 \mathrm{ppm})$, the peak molecular weight increases with monomer conversion. Extrapolation to zero monomer conversion yields $M_{\mathrm{PS}}$ of 50000 up to $60000 \mathrm{~g} \cdot \mathrm{mol}^{-1}$ for the different experiments, as described in Figure 5.38.

Number average molecular weights are found to be independent of monomer conversion and polydispersities are much higher than 2, irrespective of the applied conditions. All experimental data are reported in Appendix A2.

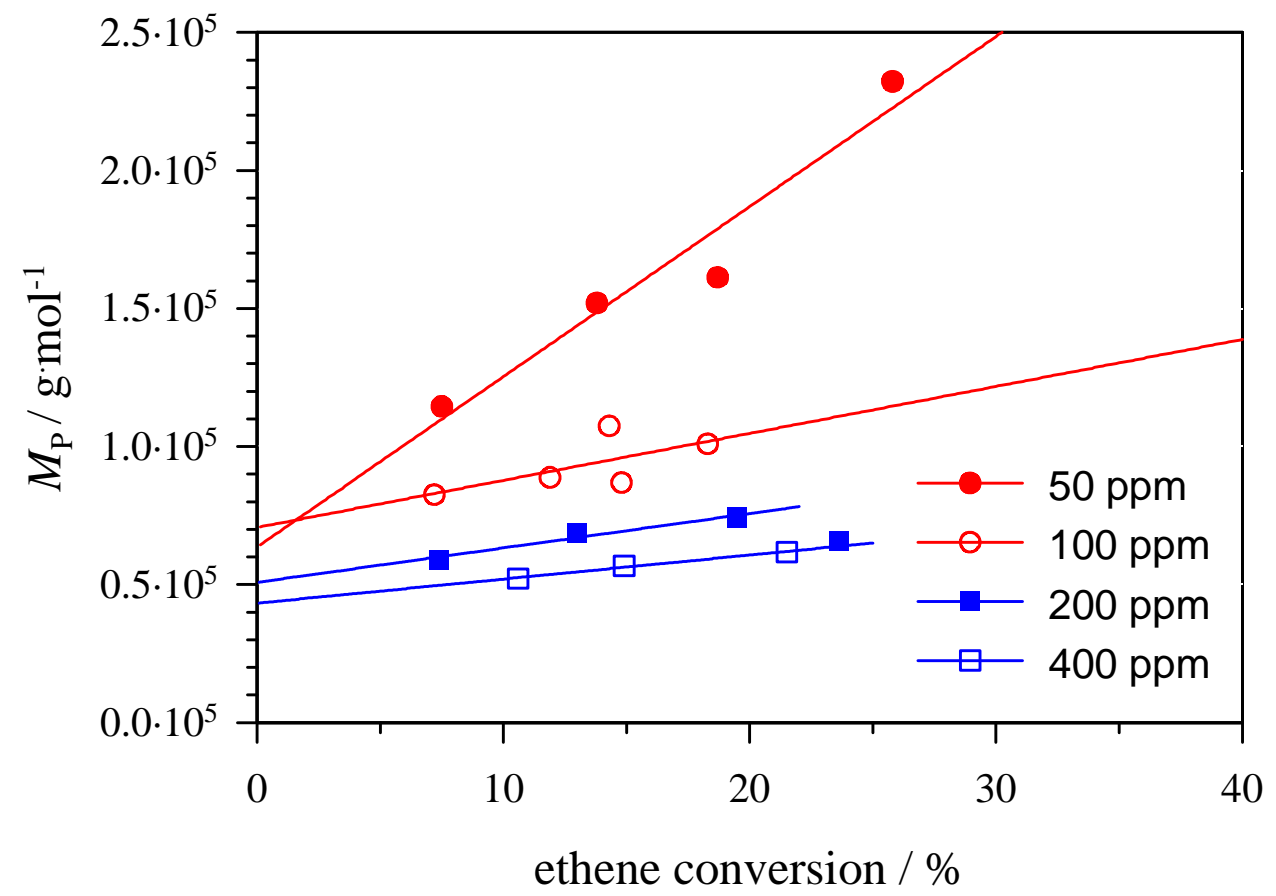

Fig. 5.38. Plot of peak molecular weight as a function of conversion for ethene polymerizations at $160^{\circ} \mathrm{C}$ and 2000 bar in the presence of different concentrations of hexyl-DPAIO.

Molecular weight distributions obtained by SEC analysis of the polymer from ethene polymerizations at different conversions in the presence of $100 \mathrm{ppm}$ of hexyl-DPAIO are shown in Fig. 5.38. At low monomer conversion, a monomodal distribution is observed. Increasing the conversion leads to the growth of a second population at high molecular 
weights inducing much higher molecular weights. This phenomenon was observed only at low alkoxyamine concentrations (50 and 100 ppm). A monomodal distribution is obtained, even at high monomer conversion, in the case of ethene polymerization with 200 and 400 ppm alkoxyamine.

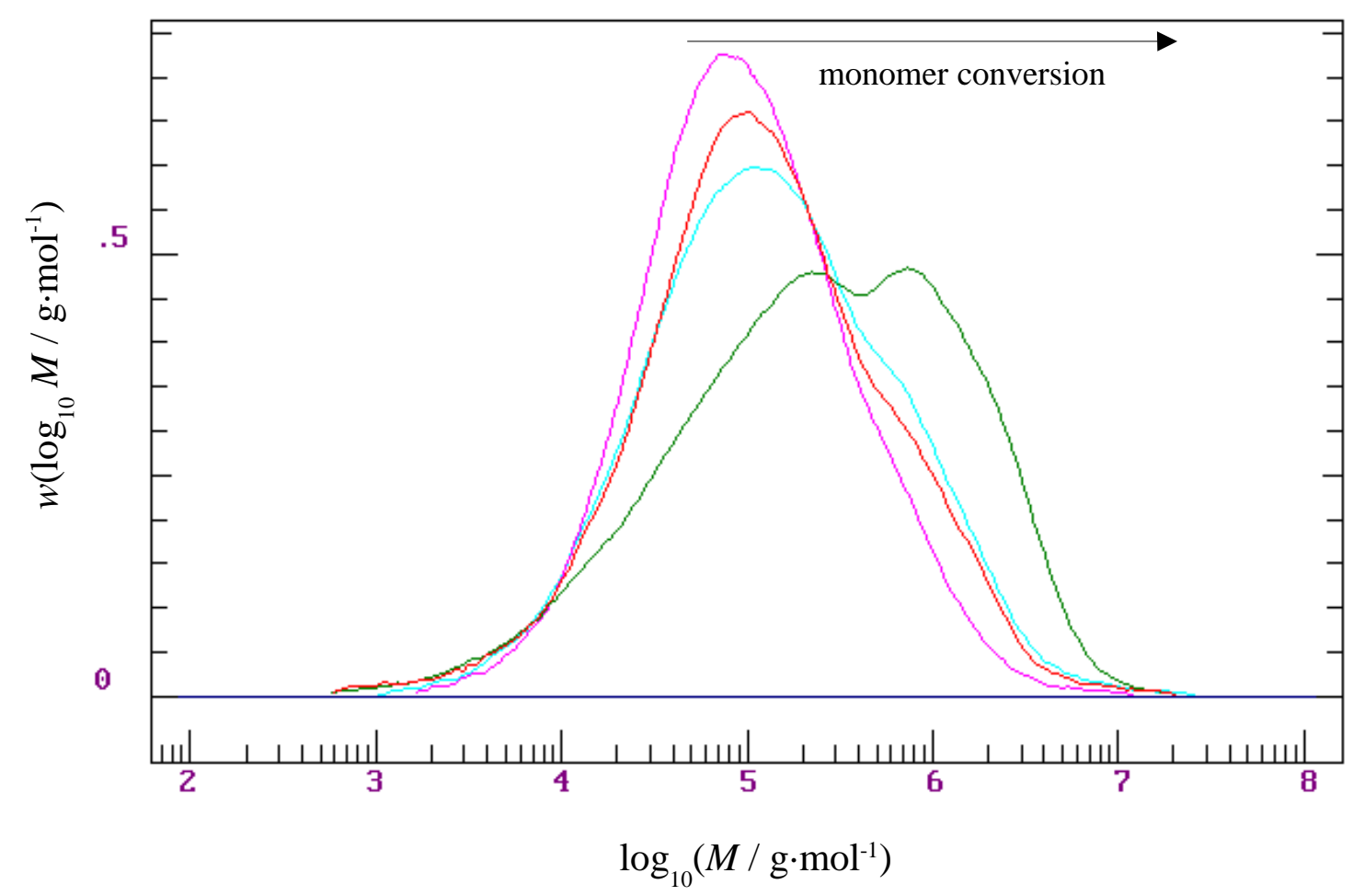

Fig. 5.39. Molecular weight distribution for ethene polymerizations at different monomer conversions at $160^{\circ} \mathrm{C}$ and 2000 bar in the presence of 100 ppm hexyl-DPAIO.

\subsection{RAFT ethene polymerization}

Ethene polymerizations were performed in the presence of the RAFT agent presented in Scheme 5.40 over a wide range of temperature at 2000 bar. This RAFT agent was chosen because of its good thermal stability and as it has already been successfully applied toward controlling vinyl acetate (VAc) polymerization ${ }^{23}$. The VAc monomer has similar reactivity as ethene. The vinyl acetate growing radical is also poorly stabilized and of low steric bulkiness and hence is both highly reactive and contributes a rather poor leaving group. The polymerization of VAc is hence completely inhibited in the presence of the preferred RAFT agents for (meth)acrylate, acrylamide and styrene polymerizations. Nevertheless xanthates and 
dithiocarbamates, which show little or no activity with these monomers, function effectively in the RAFT polymerization of vinyl acetate. Restricted reaction conditions need to be considered for controlled VAc polymerization. Low polydispersity indices are obtained at moderate conversion and the control is effective only at low molecular weights $\left(M_{\mathrm{N}} \mathrm{PVAc}\right.$ $\left.000 \mathrm{~g} \cdot \mathrm{mol}^{-1}\right)$. By translating the PVAc molecular weights into ethene units, favorable conditions should be $M_{\mathrm{N} \text { PE }}<9000 \mathrm{~g} \cdot \mathrm{mol}^{-1}$ at moderate degrees of monomer conversion.

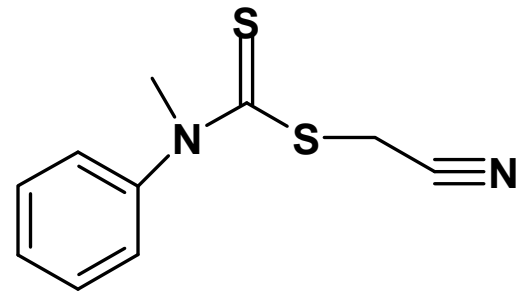

Scheme 5.40. RAFT agent used in RAFT ethene polymerization.

Ethene polymerizations were carried out at 2000 bar and at temperatures between 150 up to $180^{\circ} \mathrm{C}$ in the presence of DTBP as initiator and at high RAFT agent concentrations ([RAFT]/[E] between 100 and $400 \mathrm{ppm}$ ), in order to reach low molecular weights. The observed dependences of conversion index vs time for RAFT agent-mediated ethene polymerizations are plotted in Fig. 5.41. The conversion index $\ln \left([M]_{0} /[M]\right)$ vs time plot exhibits a straight line up to $35-40 \%$ ethene conversion. Conversions are, however, limited by the solubility of polyethylene in ethene. As usual, polymerization rate increases with temperature. The RAFT agent clearly induces a slow down of polymerization rate as compared to chemically induced (with DTBP) ethene polymerization, at $150^{\circ} \mathrm{C}$. Nevertheless RAFT ethene polymerization is very fast in comparison to RAFT vinyl acetate polymerization.

In table 5.42, $M_{\mathrm{N}}$ and $M_{\mathrm{W}}$ values from SEC are reported as a function of monomer conversion. The data is also plotted in Fig. 5.43. Molecular weights observed are quite low and polydispersities are between 2 and 3. Ip increases with monomer conversion, due to the increase of $M_{\mathrm{W}}, M_{\mathrm{N}}$ remains constant and also is insensitive toward temperature. For a given concentration of the RAFT agent, molecular weight is independent of experimental conditions, such as temperature and monomer conversion. $M_{\mathrm{N}}$ close to $10000 \mathrm{~g} \cdot \mathrm{mol}^{-1}$ are observed for polymerizations performed in the presence of $200 \mathrm{ppm}$ of RAFT agent at temperatures between 150 and $180^{\circ} \mathrm{C}$. 


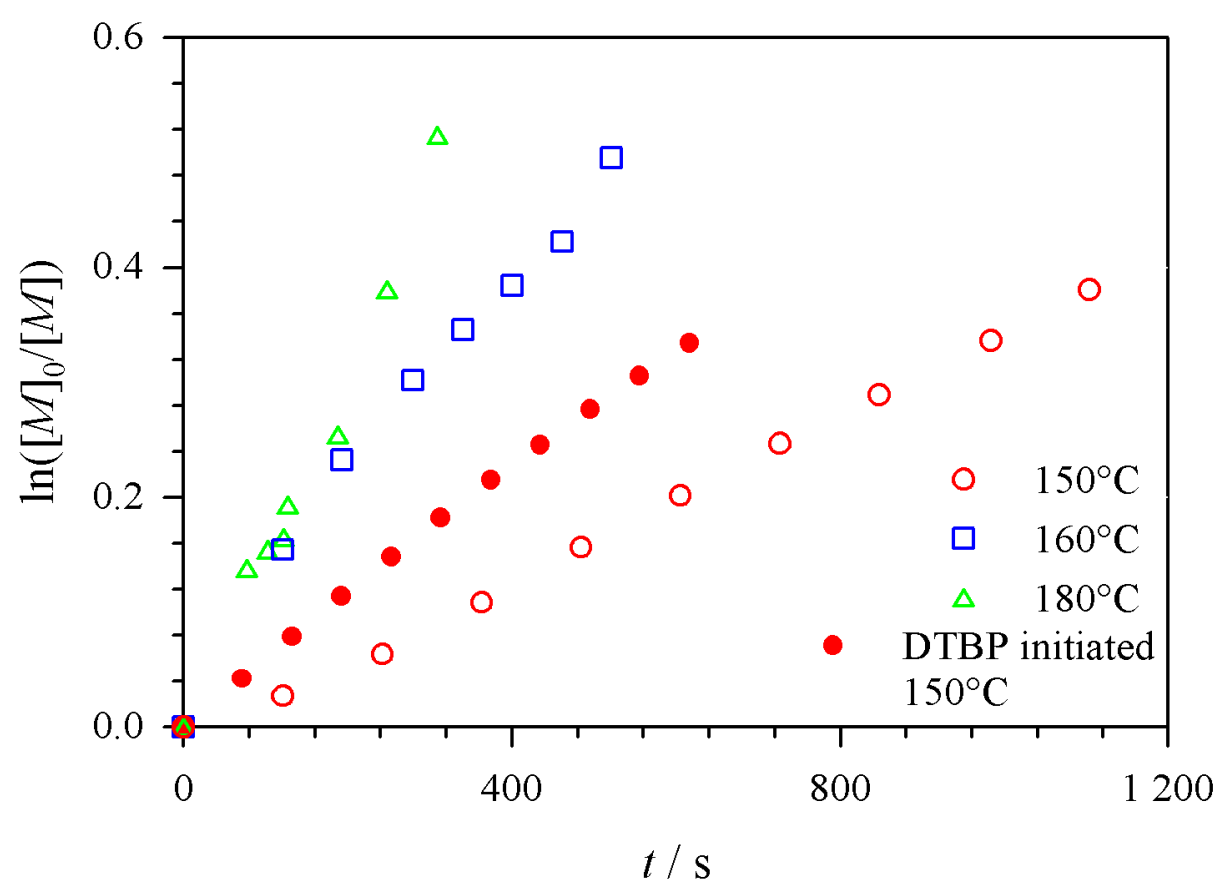

Fig. 5.41. $\ln ([M] d[M])$ vs time plot for ethene polymerizations in the presence of 200 ppm RAFT agent at 2000 bar and three temperatures. Comparison with DTBP initiated ethene polymerization at $150^{\circ} \mathrm{C}$ and 2000 bar (filled circles).

\begin{tabular}{ccccc}
\hline $\begin{array}{c}T \\
{ }^{\circ} \mathrm{C}\end{array}$ & $\begin{array}{c}\text { ethene conversion } \\
\%\end{array}$ & $\begin{array}{c}M_{\mathrm{N}} \\
\mathrm{g} \cdot \mathrm{mol}^{-1}\end{array}$ & $\begin{array}{c}M_{\mathrm{W}} \\
\mathrm{g} \cdot \mathrm{mol}^{-1}\end{array}$ & $I p$ \\
\hline \multirow{2}{*}{150} & 8.5 & 10040 & 20830 & 2.1 \\
& 18.8 & 12310 & 32330 & 2.7 \\
& 20.3 & 13250 & 27880 & 2.2 \\
& 25.4 & 12910 & 39330 & 2.4 \\
& 31.7 & 15200 & 37010 & 2.6 \\
\hline \multirow{2}{*}{160} & 14.0 & 11070 & 27120 & 2.4 \\
& 39.1 & 12870 & 35800 & 3.0 \\
\hline 180 & 10.0 & 7040 & 25730 & 3.7 \\
\hline
\end{tabular}

Table 5.42. Molecular weights and polydispersity indices at different monomer conversion for RAFT ethene polymerizations at 2000 bar and at three temperatures. $[R A F T] /[E]=200 \mathrm{ppm}$. 
Two additional ethene polymerizations were carried out in the presence of the RAFT agent. The first one was performed in the presence of a two-fold smaller RAFT concentration, $[\mathrm{RAFT}] /[\mathrm{E}]=100 \mathrm{ppm}$, at $150^{\circ} \mathrm{C}$. Two fold-higher molecular weights compared to the previous experiments were observed $\left(M_{\mathrm{N}}=24670 \mathrm{~g} \cdot \mathrm{mol}^{-1}\right.$ and $M_{\mathrm{W}}=54030 \mathrm{~g} \cdot \mathrm{mol}^{-1}$ at $18.8 \%$ conversion). An experiment was carried out at $180^{\circ} \mathrm{C}$ in the presence of a very high RAFT agent concentration, approximately $400 \mathrm{ppm}$. This experiment leads to the decomposition of ethene due to the high exothermicity of polymerization reaction under these conditions. This observation indicates that free radicals are produced from the RAFT agent.

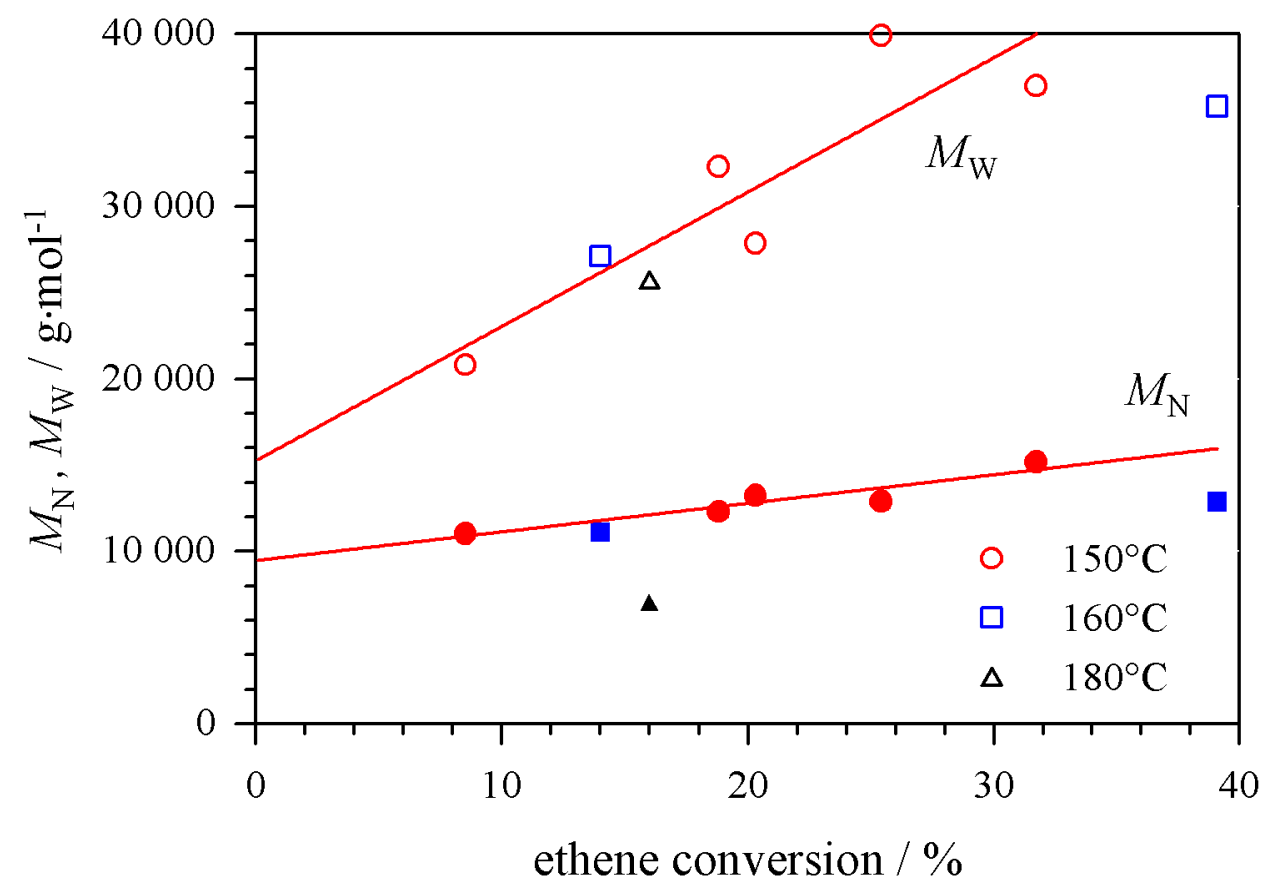

Fig. 5.43. Molecular weights as a function of monomer conversions for RAFT ethene polymerizations at 2000 bar and at three temperatures. [RAFT]/[E] $=200 \mathrm{ppm}$.

\subsection{References}

[1] M. Buback, Angew. Chem. Int. Ed. Engl. 1991, 30, 641

[2] J. Sandmann, Diploma Thesis Göttingen 1996

[3] M. Buback, L. Wittkowski, Zeitschrift für Physikalische Chemie 1999, 210, 61

[4] L. Wittkowski, Ph. D. Thesis Göttingen 1998

[5] M.B. Sderra, Ph. D. Thesis Göttingen 1997 
[6] S. Klingbeil, Ph. D. Thesis Göttingen 1995

[7] J. Sandmann, Diplom Thesis Göttingen 1996

[8] W.A. Pryor, E. H. Morkved. .H. Bickley, J. Org. Chem. 1972, 37, 1999

[9] R. Neumann, J. Acc. Chem. Res. 1972, 5, 381

[10] P.D. Barlett, R. Hiatt, J. Am. Chem. Soc. 1958, 80, 1958

[11] P.D. Barlett, C. Rüchard, J. Am. Chem. Soc. 1960, 82, 1756

[12] M.S. Karasch, A. Fono, J. Org. Chem. 1959, 24, 606

[13] J. Barton, E. Borsig, Polym. Sc. Library 61988

[14] C. Hinton, Ph. D. Thesis Göttingen 1995

[15] H. Seidl, G. Luft, J. Macromol. Sci.-Chem. 1981, A15, 1

[16] J.E. Guillet, T. Walker, M. Meyer, J. Hawk, E. Towne, Ind. Eng. Chem. Prod. Res. Develop. 1964, 3, 257

[17] H. Fischer, J. Polym. Sci., Polym. Chem., 1999, 37, 1885

[18] F. Ercole, G. Moad, C. Johnson, J. Krstina, C. Moad, E. Rizzardo, T. Spurling, S. Thang, A. Anderson, ACS Symp. 1998, 685, 332

[19] P. Tordo, Private communications

[20] D. Salomon, E. Rizzardo, P. Ccioli, U.S. Patent, 1986, 581, 429

[21] K. Matyjaszewski, ACS Symp. : Controlled Radical Polymerization 1998, 685, 2

[22] P.Tordo, D. Gigmes, Private communications

[23] E. Rizzardo, J. Chiefari, R. T. A. Mayadunne, G. Moad, S. H. Thang, ACS Symp. 2000, 768,278

[24] D. Benoit, S. Grimaldi, S. Robin, J.P.Finet, P. Tordo, Y. Gnanou, J. Am. Chem. Soc. 2000, 122 (25), 5929. 


\section{DISCUSSION}

\subsection{Study of thermal peroxide decomposition}

The measured temperature and pressure dependencies for the observed first-order decomposition rate coefficients of peroxides are quantified in the usual way in terms of activation energy and activation volume, respectively. The variation with temperature of most chemical reactions may be described by the empirical derived Arrhenius equation

$$
\ln k=\ln k_{0}-\frac{E_{a}}{R T}
$$

where $k$ is the rate coefficient, $T$ the temperature in Kelvin, and $R$ the gas constant. The term $k_{0}$ is called the pre-exponential factor, and $E_{\mathrm{a}}$ is the activation energy. $E_{\mathrm{a}}$ and $k_{0}$ are directly derived from the slope and from the y-intercept of a plot of $\ln k$ versus inverse temperature, respectively.

According to transition state theory, the pressure effect on reaction rate, at constant temperature, is described by the fundamental relationship

$$
\left(\frac{\partial \ln k}{\partial P}\right)_{T}=-\frac{\Delta V^{\neq}}{R T}
$$

where $\Delta V^{\neq}$is the activation volume. Reactions which are accelerated by pressure have a negative activation volume, whereas a positive activation volume indicates that the reaction is retarded by the application of pressure. It is from the slope of a graph $\ln k$ versus pressure that the activation volume is determined. 


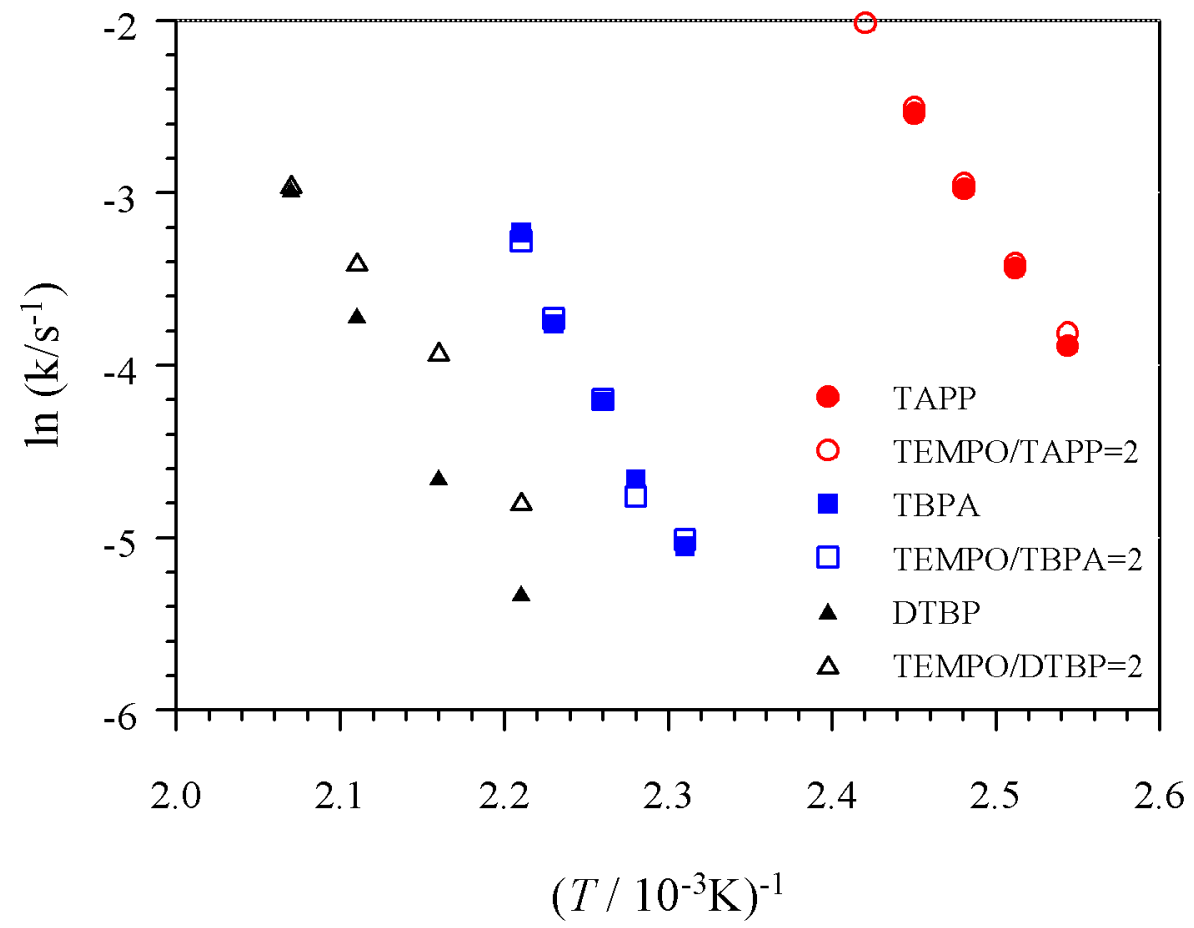

Fig. 6.1. $\quad$ Plot of $\ln k$ vs inverse temperature for the determination of activation energy for the decomposition of TAPP, DTBP and TBPA in n-heptane (for the reaction conditions see Table 6.2)

\begin{tabular}{lccc}
\hline & $E_{\mathrm{a} \text { TAPP }} / \mathrm{kJ} \cdot \mathrm{mol}^{-1}$ & $E_{\mathrm{a} \mathrm{DTBP}} / \mathrm{kJ} \cdot \mathrm{mol}^{-1}$ & $E_{\mathrm{a} \mathrm{TBPA}} / \mathrm{kJ} \cdot \mathrm{mol}^{-1}$ \\
\hline conditions & $\begin{array}{c}120-140^{\circ} \mathrm{C}, \\
500 \mathrm{bar}\end{array}$ & $\begin{array}{c}170-210^{\circ} \mathrm{C}, \\
500 \mathrm{bar}\end{array}$ & $\begin{array}{c}160-180^{\circ} \mathrm{C}, \\
\end{array}$ \\
\hline peroxide without & $120.1 \pm 3.3$ & $144.9 \pm 8 \mathrm{bar}$ \\
TEMPO & $122 \pm 8^{1}$ & $150 \pm 15^{2}$ & $148.4 \pm 3$ \\
peroxide without & & & $148 \pm 4^{3}$ \\
TEMPO (lit. data) & $121.5 \pm 4$ & $110.7 \pm 14(\mathrm{R}=2)$ & $146.0 \pm 3$ \\
peroxide with TEMPO & - & $143.5 \pm 11(\mathrm{R}=0.6)$ & - \\
\end{tabular}

Table 6.2. Activation energies for the decomposition of TAPP, DTBP, TBPA in n-heptane (for the reaction conditions see table)

In Figure 6.1 is shown the graph of $\ln k$ versus the inverse temperature for the decomposition of TAPP, DTBP and TBPA in $n$-heptane at the reaction conditions listed in Table 6.2. Also summarized in Table 6.2 are the values of activation energies calculated from the slope of $\ln k$ versus $1 / T$ data presented in Fig. 6.1. 
As the $k$ values for TAPP and TBPA do not differ in decomposition experiments with and without TEMPO, the activation energies do not differ either as is reported in Table 6.2. Decomposition rates are adequately described by first-order kinetics. As we can see from Table 6.2, the activation energies determined in this work are in excellent agreement with literature data.

The DTBP decomposition shows an other behavior in that the observed $k$ values differ significantly for situations with and without TEMPO. The reason behind this difference is most likely due to the fact that the kinetics are determined via products. The concentrations of products are influenced by reactions of intermediate radicals from DTBP decomposition with TEMPO or with species from TEMPO decomposition which can not be ignored at the fairly high temperatures of the kinetic experiments. For this reason the question whether DTBP decomposition itself is modified by the presence of TEMPO can not be safely answered. The kinetic data, however, indicate that the decomposition rates of DTBP are high enough to allow for a satisfactory initiation of ethene polymerization as usual.

The effect of pressure on the rate of DTBP decomposition at $200^{\circ} \mathrm{C}$ and at pressures of 500 up to 2500 bar is very weak and a precise method is required to quantify it. In this case, independently of the presence of nitroxide, a great scattering in data has been observed. In fact, the results appear to be strongly dependent on the integration method. In evaluating the kinetic coefficients from the TBA signal, a scattering of $k$ values taken under ostensibly the same conditions up to $15 \%$ has been observed by considering the half-band integral or the maximum peak integral ( -3 to $+3 \mathrm{~cm}^{-1}$ of the maximum). An even worse scattering has been observed by integrating the signal of acetone from the DTBP decomposition. For the study of the pressure dependence of DTBP decomposition, the scattering of integrated absorbance is so great and the pressure influence so low that the method appears to be not suitable for the quantitative measurements of such a small pressure dependence.

The quantitative analysis, via a gas chromatography coupled to the continuous flow apparatus, of products from peroxide decompositions has unfortunately not allowed to understand the difference in DTBP decomposition rates in the presence of TEMPO. The GC spectrum taken at $20^{\circ} \mathrm{C}$ allows for the characterization of DTBP (signal 1) and TEMPO (signal 2). A second signal close to TEMPO (signal 3) is due to the adduct methyl-TEMPO. 
At high temperature, the other signals in Fig. 6.3 are input to the products of DTBP decomposition, for example TBA and acetone (signals 4 and 5, respectively). Acetone is formed by $\beta$-scission of the tert-butoxy radicals, which are the primary decomposition products of DTBP. Tert-butoxy radicals can abstract an hydrogen from the solvent or other molecules or undergo a $\beta$-scission reaction resulting in methane and acetone. Heptane dimers (signal 6) are formed by hydrogen abstraction from the solvent (signal 7) and recombination of two heptyl radicals. GC spectra show that oxygen centered radicals are not trapped by TEMPO (presence of TBA even in the presence of an excess of TEMPO : see spectra at $180^{\circ} \mathrm{C}$ ). Methyl (signal 8) and heptyl radicals (carbon centered radicals) are not observed any more in the presence of an excess of TEMPO (see signal at $180^{\circ} \mathrm{C}$ ), probably due to trapping by the nitroxide which yields to alkoxyamines methyl-TEMPO (signal 3) and heptyl-TEMPO (signal 9). The GC spectra at $200^{\circ} \mathrm{C}$ plotted in Fig. 4.9 illustrates cases where all TEMPO is consumed (no signal 2) and a pronounced formation of methane (8) and heptane dimers (6) is seen. Although, as can be seen from Fig. 6.3, various GC components can be detected and assigned, the detailed kinetics of DTBP decomposition in the presence of TEMPO is not yet fully clear.

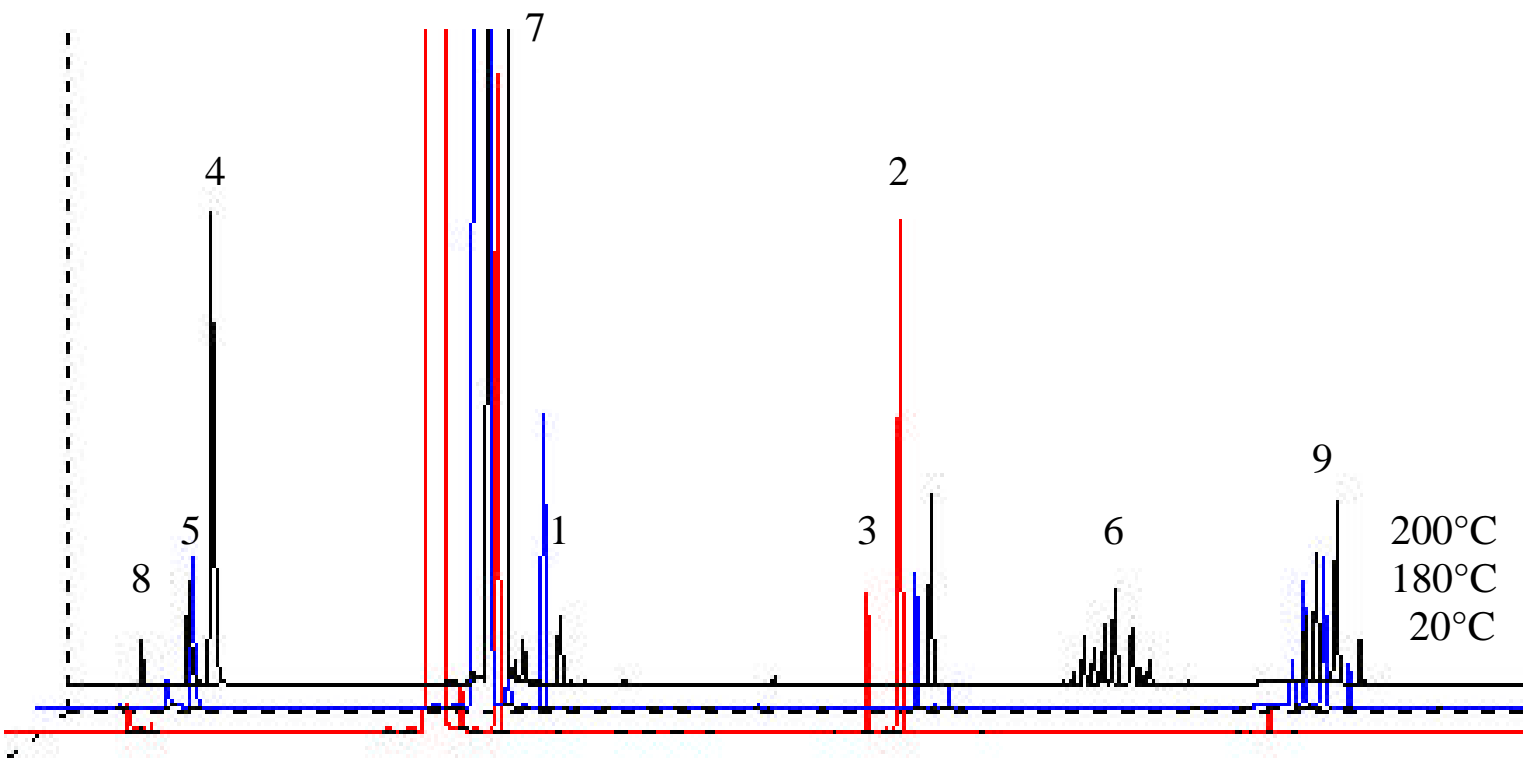

Fig. 6.3. Gas chromatography spectra of products issued from the DTBP decomposition in the presence of nitroxide $(T E M P O / D T B P=0.6)$ at 500 bar and temperatures of 20,180 and $200^{\circ} \mathrm{C}$. [DTBP] $=0.1 \mathrm{M}$. 


\subsection{Nitroxide-mediated styrene polymerization}

Experimental results presented in Section 5.3, showed the influence of pressure on polymerization rate. It was observed that high pressure does not prevent the control of styrene polymerization. As it was expected, high pressure induces an enhancement of styrene polymerization rate with TEMPO and with SG1 derivatives. The increase in polymerization rate is due to the influence of high pressure on $k_{\mathrm{p}}$ and $k_{\mathrm{t}}$, which for conventional styrene polymerization was already shown by Buback and Kuchta ${ }^{4,5}$. The pressure influence on activation and on deactivation rate coefficients of the growing radical, $k_{\mathrm{c}}$ and $k_{\mathrm{d}}$, has not been yet investigated. The coefficients will be estimated from experimental data via PREDICI ${ }^{\circledR}$ simulation. Approximate values of activation volumes have already been used in Section 4 .

The narrow polydispersities, Ip close to 1.25 at $20 \%$ conversion, at $125^{\circ} \mathrm{C}$ and 2000 bar, obtained for styrene polymerization show that the reaction is controlled over the pressure range 1 up to 2000 bar by the alkoxyamine styryl-SG1. High pressure only induces a slight lowering of polymerization control in the early stage of the reaction (up to $20 \%$ conversion), inducing higher molecular weights. Transfer and termination processes occur more frequently when the persistent radical is not available in a large excess as at the beginning of the polymerization. High pressure-induced $k_{\mathrm{p}}$ leads to the formation of high molecular weight polymer. Dead polymers may have a strong influence on number average molecular weight and polydispersity in the early period of the polymerization.

Similar phenomena are observed in case of styrene polymerization mediated with styryl-TEMPO. High pressure has only a small influence on molar mass and polydispersity. Molecular weight in TEMPO-mediated styrene polymerization at $125^{\circ} \mathrm{C}$ are closer to the theoretical predictions at low conversion than this is the case with SG1-mediated styrene polymerization. The optimum polymerization temperature, which is determined by stability and activation rate of SG1 adducts, for SG1 is in the range from 115 to $120^{\circ} \mathrm{C}$.

\subsubsection{Comparison of TEMPO and SG1 as mediators of styrene polymerization}

The plot of conversion index vs time for styrene polymerizations in the presence of styryl-TEMPO and styryl-SG1 at $125^{\circ} \mathrm{C}$ and several pressures is plotted in Fig 6.4. As it was expected, a much faster polymerization rate is observed in the presence of SG1. In fact, the 
high bulkiness of SG1 induces a higher dissociation rate coefficient of the dormant species. An increase of steric crowding at the $\mathrm{N}-\mathrm{O}$ bond induces an increase in the rate constant $k_{\mathrm{d}}$. Non cyclic compounds such as dialkoxyphosphonyl substituted dialkylalkoxyamines ${ }^{6}$ decay faster than cyclic ones ${ }^{7}$.

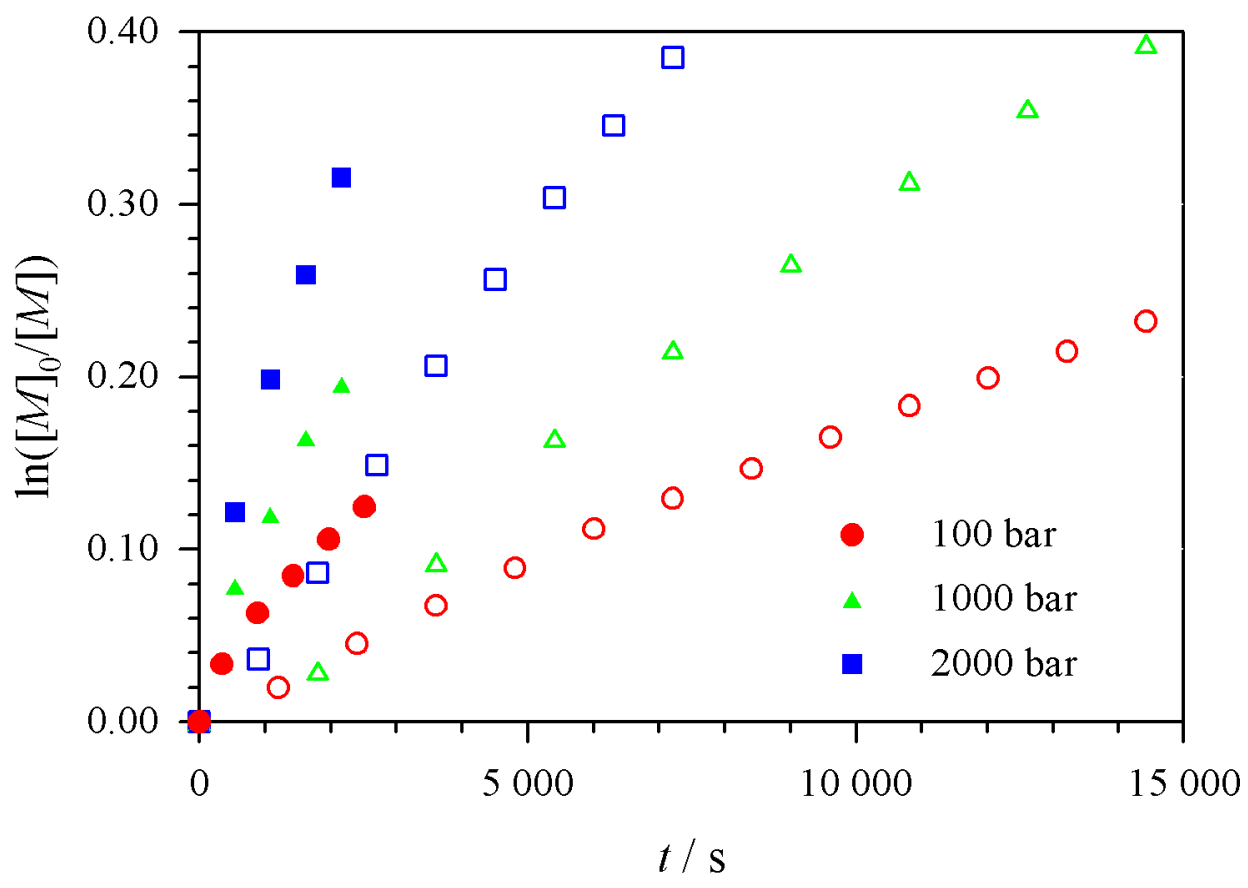

Fig. 6.4. Comparison of $\ln ([M] d /[M])$ vs time plots for styrene polymerizations in the presence of 0.11 mol\% styryl-TEMPO (open points) and styryl-SG1 (filled points) at $125^{\circ} \mathrm{C}$ and at three pressures.

The plot of the conversion index vs time of styrene polymerization in the presence of SG1 and TEMPO exhibits a downward curvature. $\ln \left([M]_{0} /[M]\right)$ follows a time dependence of the type $\mathrm{a}+\mathrm{b} \cdot t^{\mathrm{c}}$ were $\mathrm{c}$ is close to $2 / 3$. Thus the controlled styrene polymerizations under pressure follow the characteristic rate law described by Fischer (see Equation 2.9), which indicates that the persistent radical effect (see Section 2.3) occurs, and termination can not be neglected.

It should be noted, that the combination of high pressure and a bulky nitroxide such as SG1 induces an approximately 9 times faster polymerization rate as compared to the classical TEMPO-mediated styrene polymerization at 1 bar.

The plot of the molar mass evolution with time in Fig. 6.5 shows that higher molecular weights are obtained in the presence of SG1. The calculated efficiency factors, which is defined as fractions $M_{\mathrm{N}} / M_{\mathrm{N}}$ theory with $M_{\mathrm{N}}$ theory referring to the theoretical number average 
molecular weight estimated from mediator concentration and from monomer concentration, are close to 1 and to 0.53 for styryl-TEMPO and styryl-SG1, respectively. The fairly high temperature probably causes some SG1 thermal decomposition and reduces the efficiency factor for styryl-SG1 mediated styrene polymerization. The rates of thermal decomposition of nitroxides are reported in Section 5.5.3. The rapid dissociation of the dormant species and the lower thermal stability of SG1 induce a loss of growing radicals via termination and transfer processes. SG1 requires a lower temperature for providing good control.

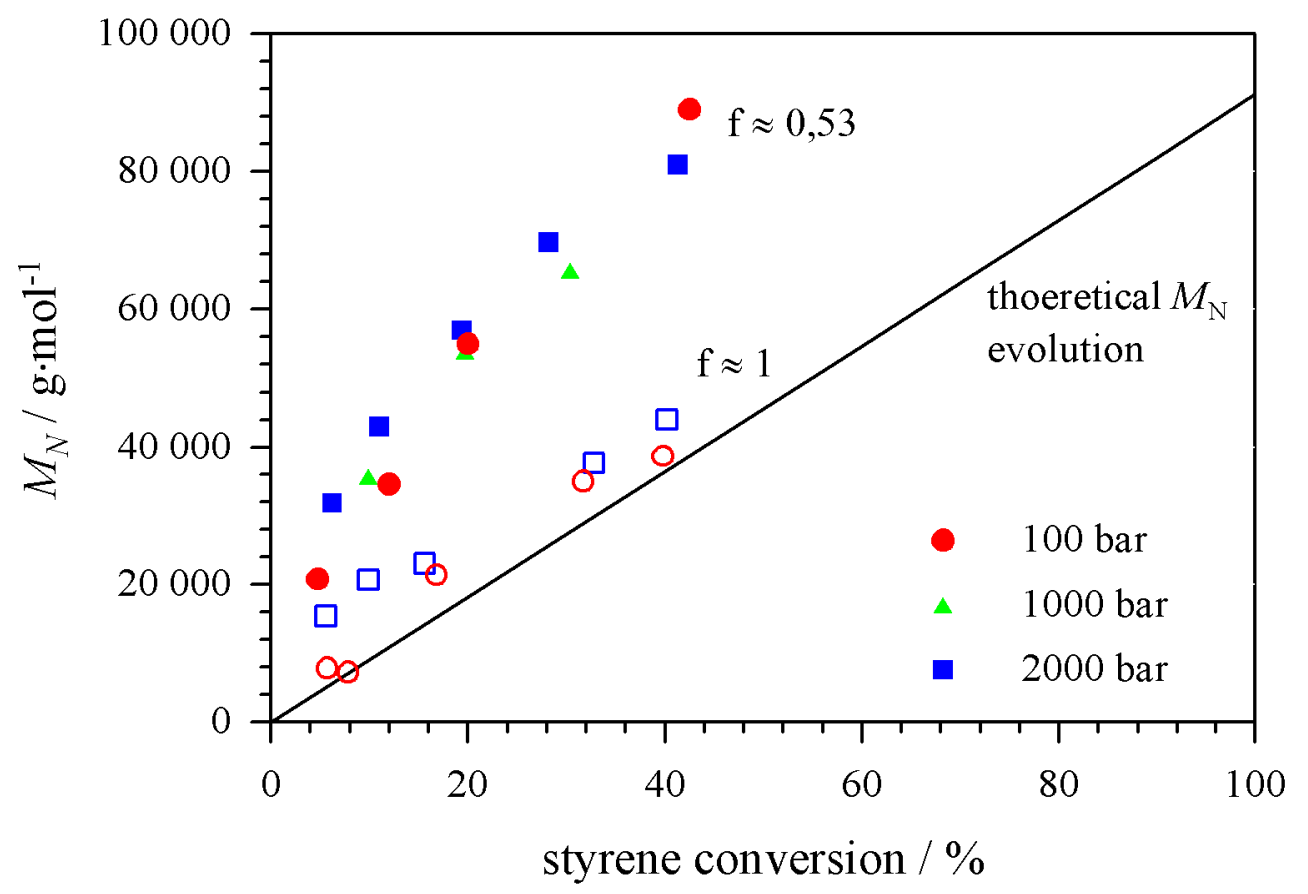

Fig. 6.5. Comparison of molar mass evolutions for styrene polymerizations in the presence of 0.11 mol\% styryl-TEMPO (open points) and styryl-SG1 (filled points) at $125^{\circ} \mathrm{C}$ and at pressures up to 2000 bar.

\subsubsection{DPAIO-mediated styrene polymerization}

DPAIO-mediated styrene polymerization shows an intermediate polymerization rate which is lower than the rate for SG1-mediated but much faster than for TEMPO-mediated styrene polymerization. This was not expected because of the high stability of the alkoxyamine styryl-DPAIO. Experimental data, presented in Section 5.3.3, showed that the system styrene-DPAIO does not yield a linear kinetic plot of $\ln \left([M]_{0} /[M]\right)$ vs time and no linear $M_{\mathrm{N}}$ evolution with conversion. Molecular weights are dependent on pressure, around 
$40000 \mathrm{~g} \cdot \mathrm{mol}^{-1}$ and $130000 \mathrm{~g} \cdot \mathrm{mol}^{-1}$ at 100 and $2000 \mathrm{bar}$, respectively, irrespective of the monomer conversion. Molar masses appear to be controlled by propagation and termination reactions. High pressure is expected to induce an increase of the propagation rate and a decrease of the termination rate, as in conventional free-radical styrene polymerization. Assuming that styryl-DPAIO cleavage temperature is close to $120^{\circ} \mathrm{C}$, styrene polymerization was performed at higher temperature $\left(140^{\circ} \mathrm{C}\right)$. Unfortunately, even at $140^{\circ} \mathrm{C}$, with different initiation systems (AIBN/DPAIO or cyano-isopropyl - DPAIO, that cleavage temperature of which is close to $70^{\circ} \mathrm{C}$ ) and with or without nitroxyl radical excess, styrene polymerization is not controlled. This is probably due to the high stability of the adduct styryl-DPAIO which requires high temperature to be dissociated. At $125^{\circ} \mathrm{C}$, the low cleavage rate does not allow for the persistent radical effect to take place and at $140^{\circ} \mathrm{C}$ the spontaneous thermal styrene polymerization has a significant influence on molecular weights. In both cases, spontaneous thermal polymerization will induce high polydispersities and constant molecular weights with monomer conversion.

\subsubsection{Effect of the initiating system}

Styrene polymerizations were carried out in the presence of a classical initiator such as BPO and an excess of TEMPO (compared to BPO) in order to avoid formation of high molecular weight polymer in the early period of the styrene polymerization. The pressure influence on conversion index and on the molecular weight evolution for styrene polymerization at $125^{\circ} \mathrm{C}$ in the presence of different initiation systems are illustrated for the pressure range from 1 up to 2000 bar in Figs. 6.6 and 6.7. The initiation system has no influence on the evolution of the molar masses. Even for a ratio [TEMPO]/[BPO] of 2.5, molecular weights between 9000 and $15000 \mathrm{~g} \cdot \mathrm{mol}^{-1}$ are observed at $10 \%$ monomer conversion, which are 2 or 3 times larger than the expected value of $4700 \mathrm{~g} \cdot \mathrm{mol}^{-1}$. An excess of nitroxyl radical then does not avoid the formation of high molecular weight polymer in the earlier stage of the polymerization. 


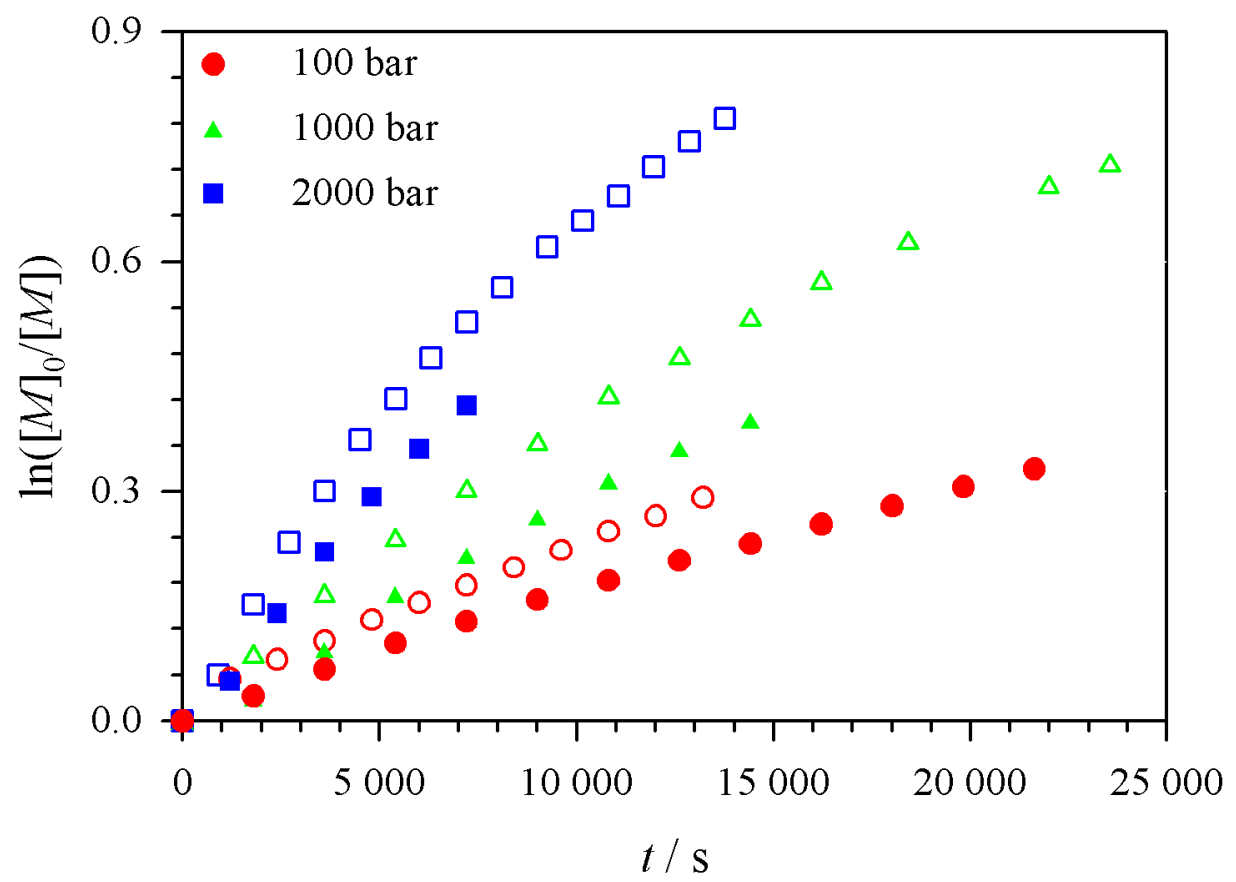

Fig. 6.6. $\quad$ Pressure influence on $\ln ([M] d[M])$ vs time plots for styrene polymerizations in the presence of 0.11 mol\% styryl-TEMPO (open points) and $0.055 \mathrm{~mol} \% \mathrm{BPO}$ $([T E M P O] /[B P O]=2.5)($ filled points $)$ at $125^{\circ} \mathrm{C}$.

On the other hand, polymerization rates are dependent on relative initiator-mediator concentrations. Fig. 6.6. shows that, irrespective of the applied pressure, the polymerization rate is lower when an excess of TEMPO (over the initiator) is used. The rate of polymerization of styrene in CRP seems to be dependent on the excess of free nitroxide over growing radicals. This phenomenon has already been observed by Georges et al ${ }^{8}$. They showed that the rate of styrene CRP is dependent on the excess of free nitroxide in the reaction mixture but the number of polymer chains is still predominantly determined by the amount of initiator used. 


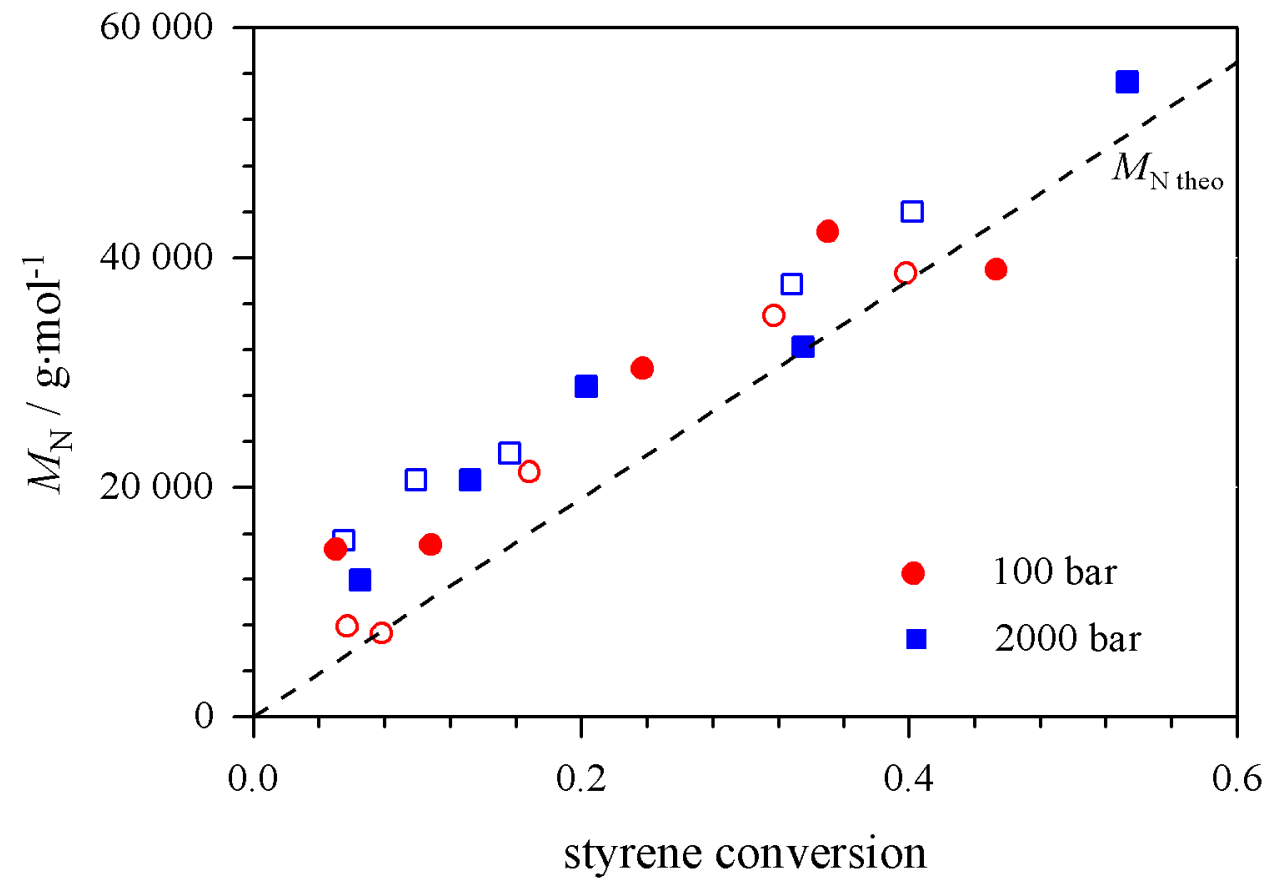

Fig. 6.7. $\quad$ Pressure influence on molecular weight evolution with monomer conversion for styrene polymerizations in the presence of 0.11 mol\% styryl-TEMPO (open points) and $0.055 \mathrm{~mol} \% \mathrm{BPO}([\mathrm{TEMPO}] /[\mathrm{BPO}]=2.5)$ (filled points) at $125^{\circ} \mathrm{C}$. The dashed line represents the theoretical $M_{N}$.

\subsubsection{Advantages of high pressure in styrene CRP}

The major advantage of high pressure, as shown in Fig. 5.9 and 5.12 is that high pressure allows for a much faster polymerization rate and then reduces polymerization time. Assuming pressure to have only a weak influence on the alkoxyamine equilibrium constant $K$ (see Section 6.2.4), the increase in polymerization rate is primarily due to the pressure influence on $k_{\mathrm{p}}$ and $k_{\mathrm{t}}$. Increasing $P$ from 1 to 2000 bar enhances styrene polymerization rate (in the presence of TEMPO or SG1) by about a factor four.

Georges et al. ${ }^{9,10}$ reported that TEMPO forms a stable adduct at $T<120^{\circ} \mathrm{C}$ and thus plays the role of a radical scavenger. Nevertheless, styrene polymerizations were carried out at low temperature and at high pressure. Higher $k_{\mathrm{p}}$ and lower $k_{\mathrm{t}}$ should allow for a reasonable polymerization rate at $100^{\circ} \mathrm{C}$ in the presence of SG1 and at $115^{\circ} \mathrm{C}$ with TEMPO. Fig 6.8 shows the $\ln \left([M]_{0} /[M]\right)$ plot with time for styrene polymerizations in the presence of SG1 at $100^{\circ} \mathrm{C}$ and 2000 bar at two alkoxyamine concentrations. The polymerization reaches about 
$50 \%$ conversion in 10 hours at $100^{\circ} \mathrm{C}$. Higher alkoxyamine concentration induces only a weak increase of the polymerization rate. Molar mass and polydispersity index evolutions are reported in Fig. 6.9. Even at $100^{\circ} \mathrm{C}$, the cleavage of the alkoxyamine seems to occur which allows to obtain linear evolutions of molecular weight with monomer conversion. Low polydispersities ( $I p=1.3$ above $30 \%$ conversion) are obtained, independently of the alkoxyamine concentration. High alkoxyamine concentrations, however allow for a slightly better control of the polymerization : at low conversion and with low adduct concentrations, higher molecular weights are obtained and the extrapolation to zero conversion does not pass through the origin. At $10 \%$ conversion, Ip is close to 1.7 compared to 1.4 at the twofold higher adduct concentration. This phenomenon, already reported in Section 6.2.3 can not be avoided, even by introducing a large excess of nitroxyl radical to rapidly induce the persistent radical effect. Thus high alkoxyamine concentration is required to obtain molecular weights at low conversion close to the theoretically expected one.

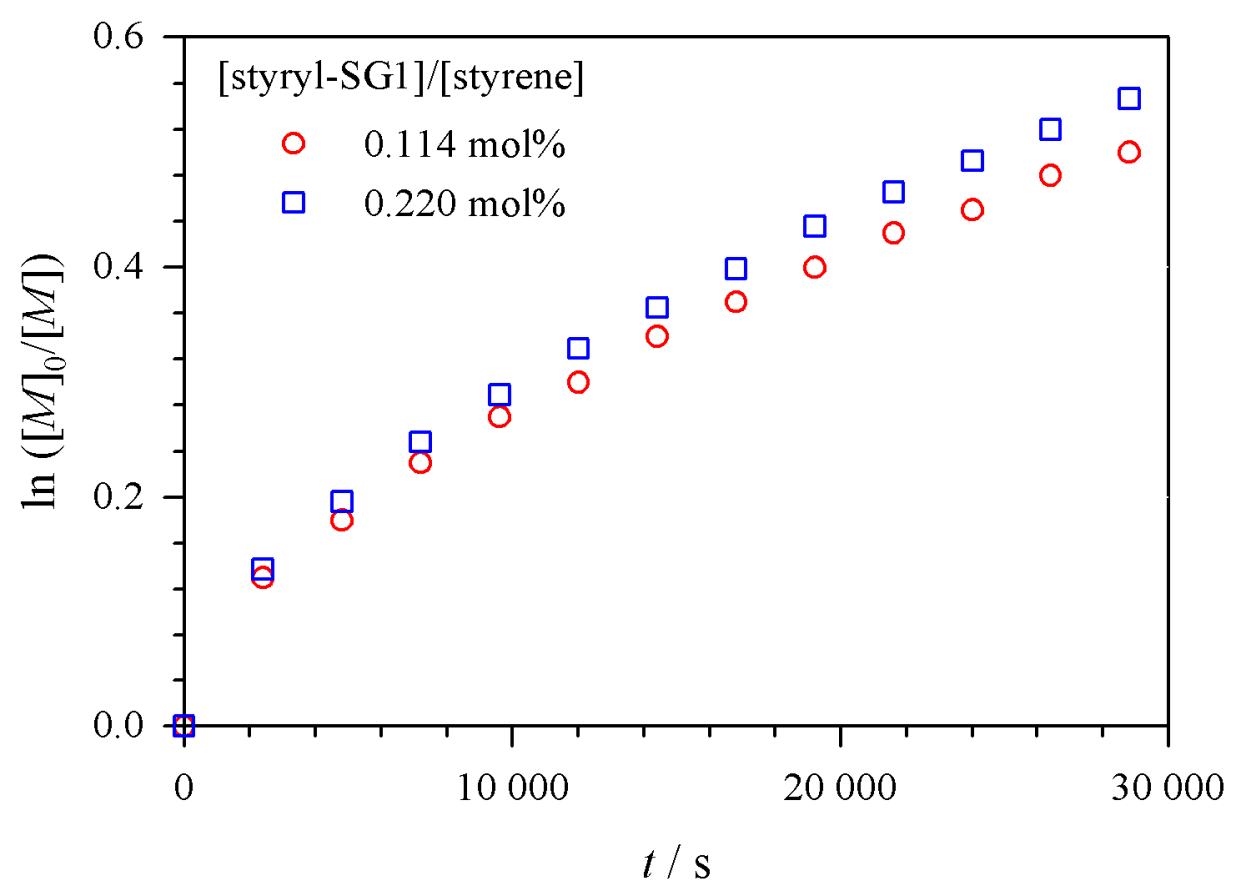

Fig. 6.8. $\ln ([M] d[M])$ vs time plot for styryl-SG1-mediated styrene polymerizations at $100^{\circ} \mathrm{C}$ and $2000 \mathrm{bar}$.

Lower polymerization temperature presents the twofold advantage of decreasing both self initiation and transfer reactions which have an unfavorable effect on polymerization control. 


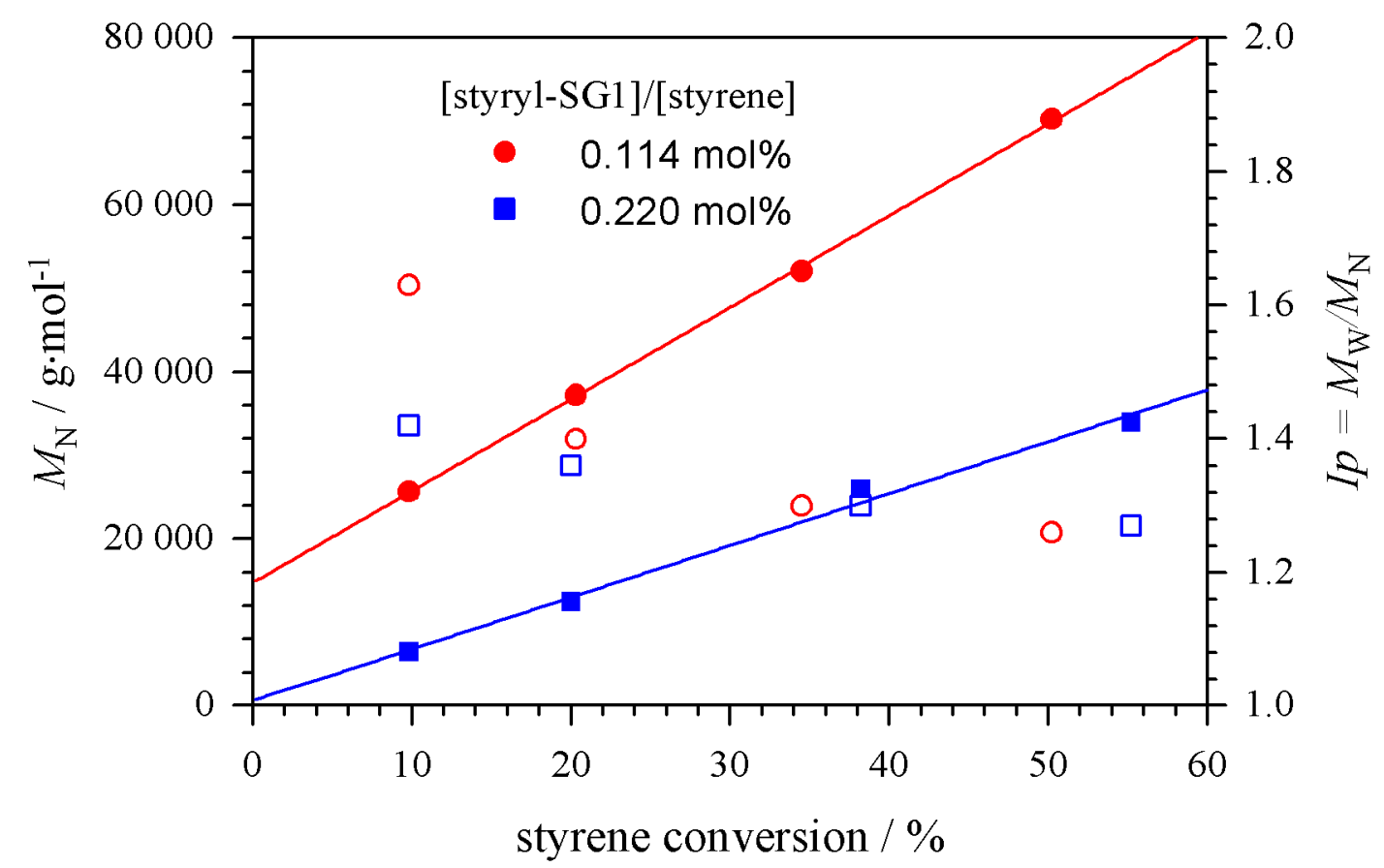

Fig. 6.9. $\quad$ Plot of molecular weight (filled points) and polydispersity index (open points) as a function of monomer conversion for styryl-SG1-mediated styrene polymerizations at $100^{\circ} \mathrm{C}$ and 2000 bar.

Rate enhancement upon the addition of an initiator during the course of the polymerization ${ }^{11}$ has already been reported for the controlled styrene polymerization with TEMPO. The same effect is observed upon adding camphrosulfonic acid ${ }^{9,10}$ to consume the stable radicals which accumulate during polymerization. High pressure appears to be an other means to enhance polymerization rate. Styrene polymerization, carried out in the presence of styryl-TEMPO at $115^{\circ} \mathrm{C}$ and 2000 bar, reaches about $50 \%$ conversion in 9 hours. Linear molar mass evolutions and low polydispersities are plotted in Fig. 6.10 and show the good control of styrene polymerization with TEMPO at $115^{\circ} \mathrm{C}$, that is even at low temperature. The cleavage of the alkoxyamine thus seems to occur at a sufficient rate even at $115^{\circ} \mathrm{C}$ and 2000 bar. Control is improved by low self-initiation at $115^{\circ} \mathrm{C}$. 


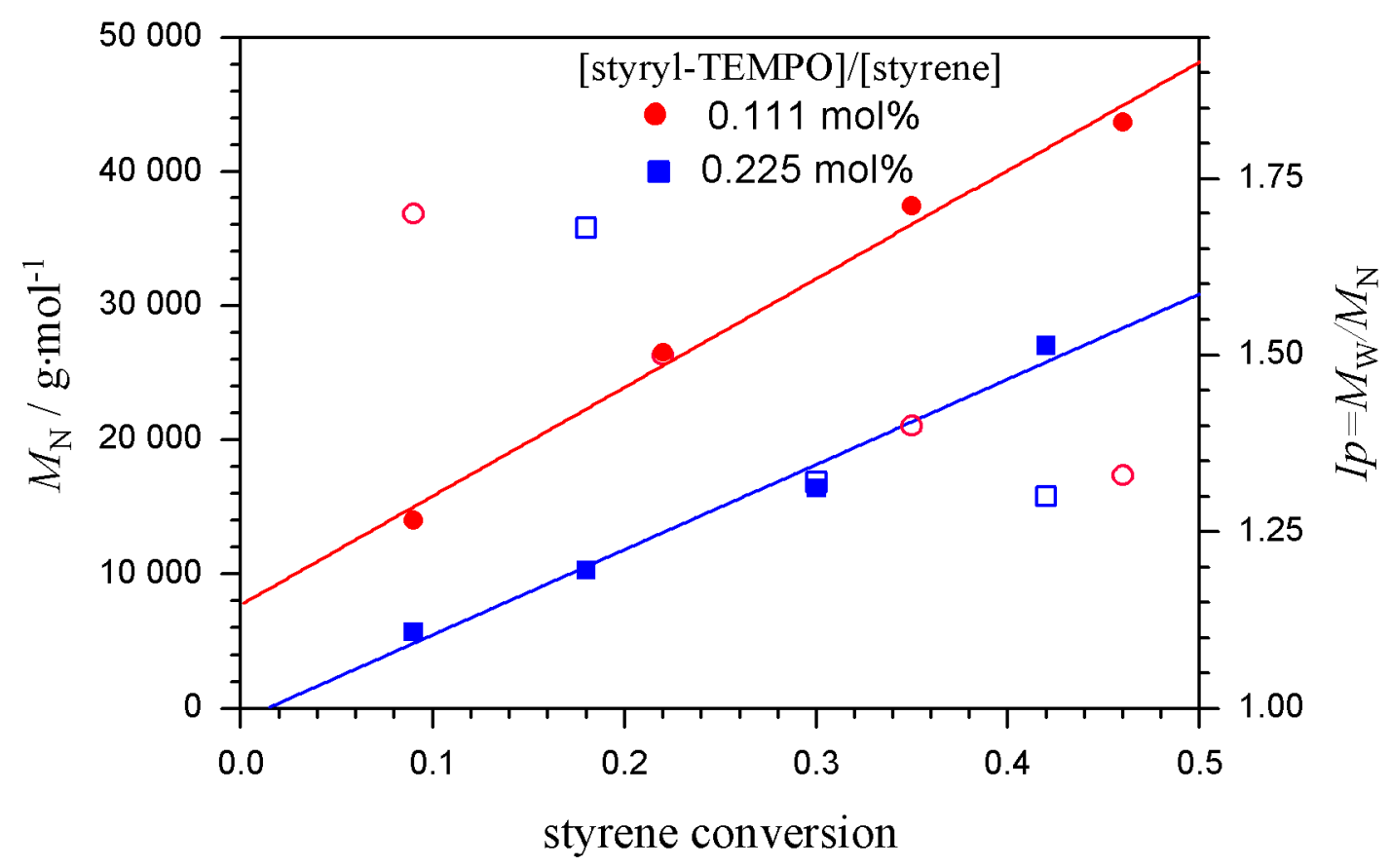

Fig. 6.10. $\quad$ Plot of molecular weight (filled points) and polydispersity index (open points) as a function of monomer conversion for styryl-TEMPO-mediated styrene polymerizations at $115^{\circ} \mathrm{C}$ and 2000 bar.

\subsubsection{Effect of low initiator concentration on styrene CRP}

Most of the controlled polymerizations described in the literature are carried out in the presence of high initiator concentrations which induce quite low molecular weights in a range from 30000 up to $50000 \mathrm{~g} \cdot \mathrm{mol}^{-1}$. Such molecular weights are reported in Section 6.2.4 to occur in styrene polymerizations with TEMPO and with SG1 under high pressure. Tordo et al. $^{12}$ argued that in controlled styrene polymerizations with SG1 high molar masses until 100 $000 \mathrm{~g} \cdot \mathrm{mol}^{-1}$ and more may be reached, but such high values have not been yet found. Boutevin et al. $^{13}$ reported the molecular weight evolution as a function of monomer conversion of SG1-mediated styrene polymerization. The molar mass evolution reported was not linear at high conversion and reached an upper limiting value close to $70000 \mathrm{~g} \cdot \mathrm{mol}^{-1}$. The observed phenomenon was not discussed. Similar observations were made by Tordo ${ }^{14}$. Therefore, within the present work, styrene polymerizations were performed at low initiator concentrations and the pressure influence was studied, especially at high conversion where high molecular weights may be obtained. 
The polymerization of styrene in the presence of alkoxyamine styryl-SG1 at $125^{\circ} \mathrm{C}$ has been described in Section 5.3.1 and high molar masses (70 $000 \mathrm{~g} \cdot \mathrm{mol}^{-1}$ ) have been reached at $30 \%$ conversion. Further polymerizations were performed at $120^{\circ} \mathrm{C}$ in the presence of two low alkoxyamine concentrations in order to reach higher molecular weights at reasonable conversion (up to $60 \%$ monomer conversion). The MWDs of polymer obtained for different monomer conversions for styryl-SG1-mediated styrene polymerization at $125^{\circ} \mathrm{C}$ at two pressures, 50 and 2000 bar, are plotted in Fig. 6.11. High pressure has only a small influence on molecular weight distribution up to $40 \%$ styrene conversion : similar MWDs are obtained up to $M_{\mathrm{N}}$ is $80000 \mathrm{~g} \cdot \mathrm{mol}^{-1}$ at 50 and $2000 \mathrm{bar}$. At higher styrene conversion, e.g. at $55 \%$, the MWD obtained at 50 bar is not further shifted any more to high molar masses but is much broader than the MWD obtained at 2000 bar.

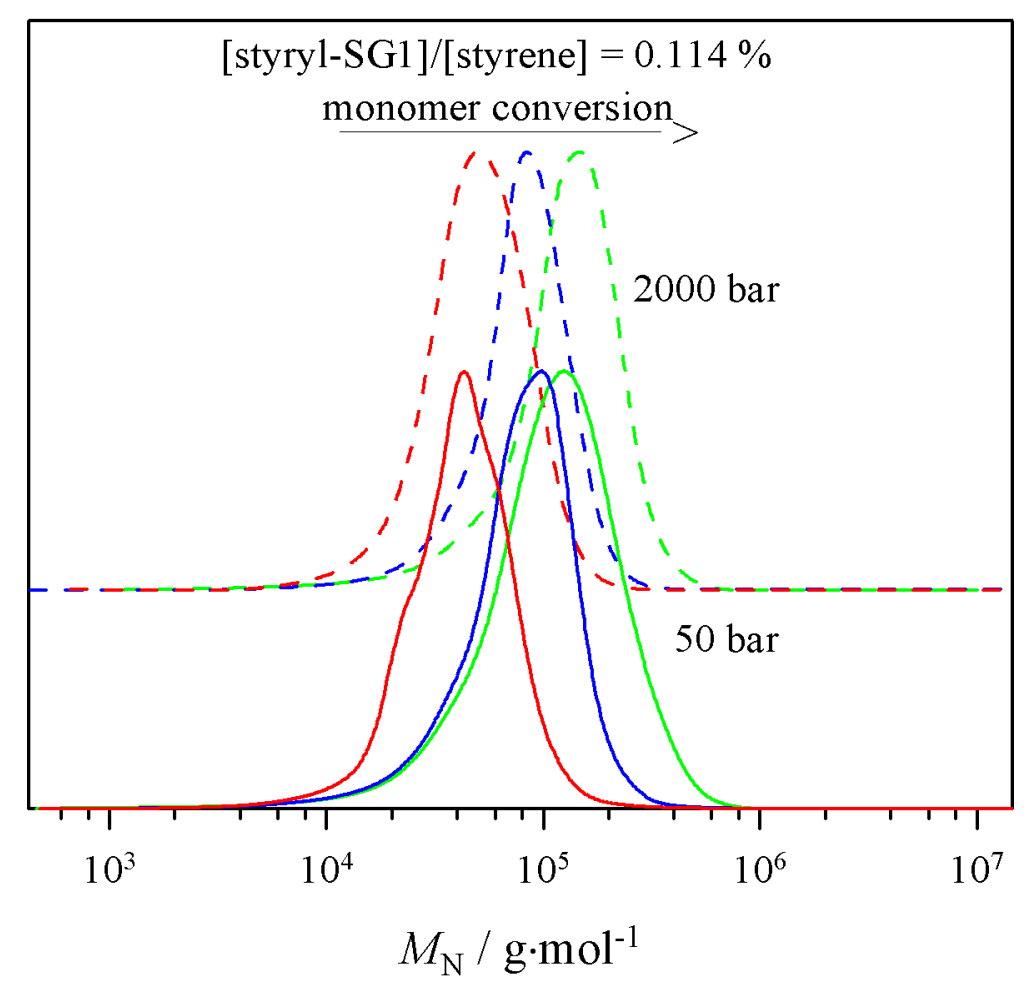

Fig. 6.11. Experimental molecular weight distributions at several monomer concentrations $(10,30$ and $55 \%)$ for styrene polymerizations at $120^{\circ} \mathrm{C}$ in the presence of 0.114 mol\% styryl-SG1 at 50 (solid lines) and 2000 bar (dashed lines).

The plot of molecular weight as a function of monomer conversion for styrene polymerizations carried out at different styryl-SG1 concentrations is reported in Fig. 6.12. 
Polydispersity vs monomer conversion is plotted in insert. Styryl-SG1-mediated polymerization under high pressure allows the production of high molecular weight material $\left(>10^{5} \mathrm{~g} \cdot \mathrm{mol}^{-1}\right.$ ) with narrow polydispersity. Polymerizations performed at low pressure do not yield such high molar masses. At lower pressure, the number average molecular weight, $M_{\mathrm{N}}$, independently of the alkoxyamine concentration, reaches a upper limiting value of about $90000 \mathrm{~g} \cdot \mathrm{mol}^{-1}$. Polydispersity indices are much lower for high pressure polymerizations, 1.3 and 1.55 at 2000 and 50 bar, respectively. The molecular weight limitation is probably due to the relevant effect of dead polymer on the polydispersity. The accumulation of radicals from spontaneous initiation, due to high polymerization time, combined with the irreversible deactivation of the dormant species during the polymerization, thus induces a decrease of persistent radical concentration, and thus an increase of the concentration of dead polymer. High pressure allows higher polymerization rate without relevant increase of spontaneous thermal initiation of styrene (see Section 4.1.4). High conversions are reached four or five times faster at 2000 bar as compared to 1 bar.

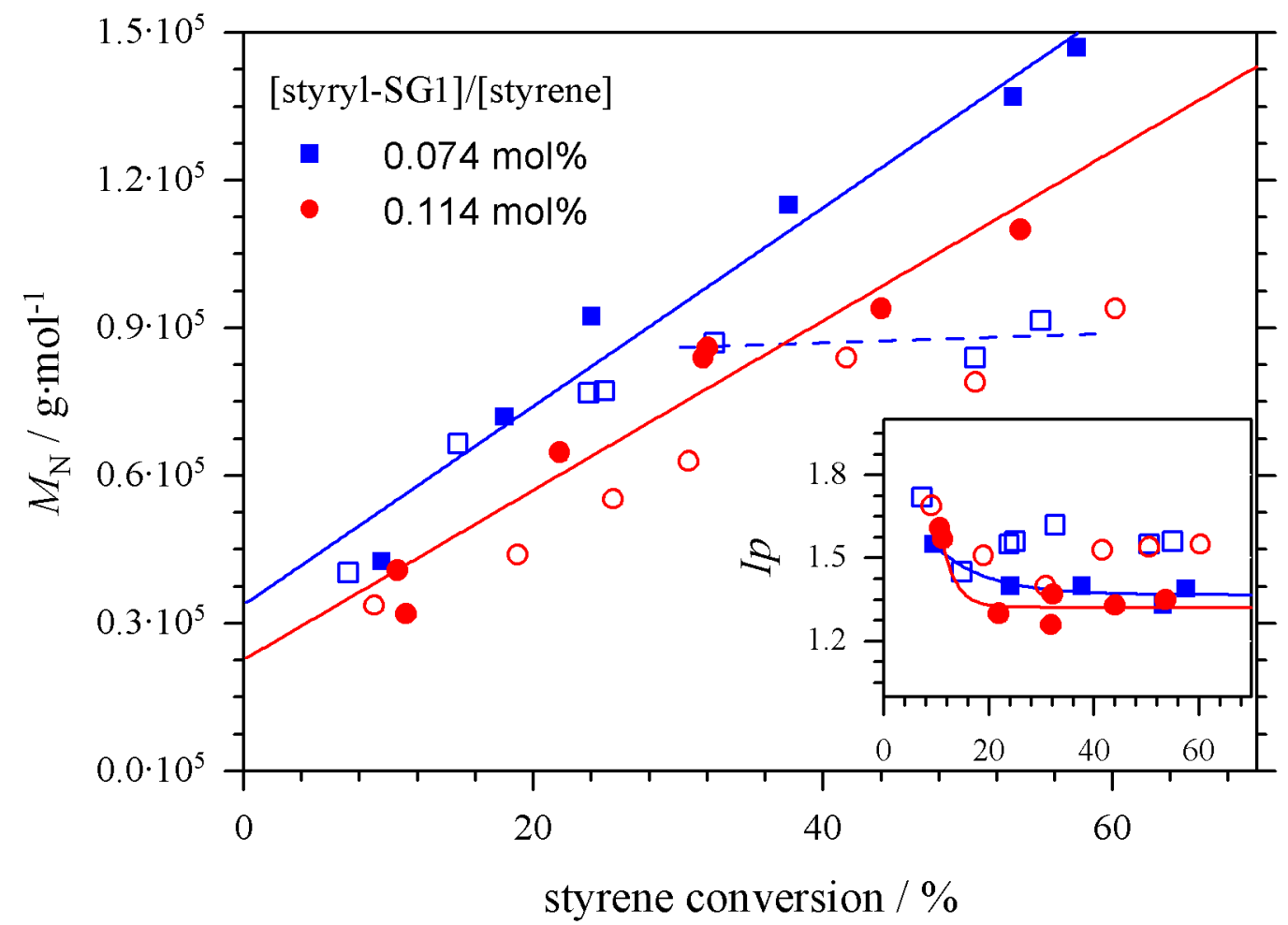

Fig. 6.12. Plot of molecular weight and polydispersity index (insert) as a function of monomer conversion for styrene polymerizations in the presence of low styryl-SG1 concentrations at 50 (open points) and 2000 bar (filled points) and $120^{\circ} \mathrm{C}$. 
Experimental molar masses were found to be around $20 \%$ higher than the values predicted from the alkoxyamine concentrations in styrene $\left(M_{\mathrm{N}}\right.$ theo are 110000 and $90000 \mathrm{~g} \cdot \mathrm{mol}^{-1}$ at $70 \%$ conversion for the experiments at 0.074 and $0.114 \%$ alkoxyamine, respectively). The discrepancy is due to the fact that the persistent radical effect does not work in the early period of the polymerization. The estimation of the amount of living polymer at different monomer conversions would be helpful toward understanding the mechanism of broadening of the MWD. Dormant species and dead polymer will be estimated during the course of the polymerization via the program PREDICI ${ }^{\circledR}$.

\subsubsection{Simulation of TEMPO-mediated styrene polymerization under high pressure}

Styrene polymerizations have been intensively investigated and rate coefficients for most of the involved reactions have become available in the literature. Temperature and pressure dependences of kinetic coefficients are presented in Section 4.1.2. A major problem concerns the determination of invividual rate coefficients $k_{\mathrm{c}}$ and $k_{\mathrm{d}}$. The simulations show that the equilibrium constant $K=k_{\mathrm{d}} / k_{\mathrm{c}}$ rather than the absolute value of the rate constants $k_{\mathrm{c}}$ and $k_{\mathrm{d}}$ will determine the polymerization kinetics over the time scale of interest (see Section 4.2.7).

The pressure influence on activation and deactivation rate coefficients $k_{\mathrm{d}}$ and $k_{\mathrm{c}}$ is estimated by fitting the experimental data. The prediction concerning the pressure dependence of $k_{\mathrm{c}}$ made in Section $4.4\left(\Delta V^{\neq}\left(k_{\mathrm{c}}\right)=\Delta V^{\neq}\left(k_{\mathrm{t}}\right)=22 \mathrm{~cm}^{3} \cdot \mathrm{mol}^{-1}\right)$ was verified by the simulations. $k_{\mathrm{d}}$ at $125^{\circ} \mathrm{C}$ was found to be $1.0 \cdot 10^{-3} \mathrm{~L} \cdot \mathrm{mol}^{-1} \cdot \mathrm{s}^{-1}$ at 1 bar and $6.2 \cdot 10^{-4} \mathrm{~L} \cdot \mathrm{mol}^{-1} \cdot \mathrm{s}^{-1}$ at $2000 \mathrm{bar}$. This induces an activation volume close to $8 \mathrm{~cm}^{3} \cdot \mathrm{mol}^{-1}$ for the dissociation reaction. As shown in Figure 6.13, these rate coefficients data allow for a good simulation of the polymerization rate and the molecular weight evolution with monomer conversion. The constant $K=k_{\mathrm{d}} / k_{\mathrm{c}}$ has been varied but no better fit is obtained. Upon varying $k_{\mathrm{c}}$ and $k_{\mathrm{d}}$ by the same factor ( $K$ remains constant) within a certain range allows to adequatly simulate the experimental results. As $k_{\mathrm{c}}$ and $k_{\mathrm{d}}$ have not been directly measured, only the pressure dependence of $K$ will be condidered. In this case, pressure induces a very low increase of $K$ from $1.1 \cdot 10^{-9}$ to $2.6 \cdot 10^{-9}$ in going from 1 to 2000 bar, due to the higher pressure dependence of $k_{\mathrm{c}}$ than the one of $k_{\mathrm{d}}$. 

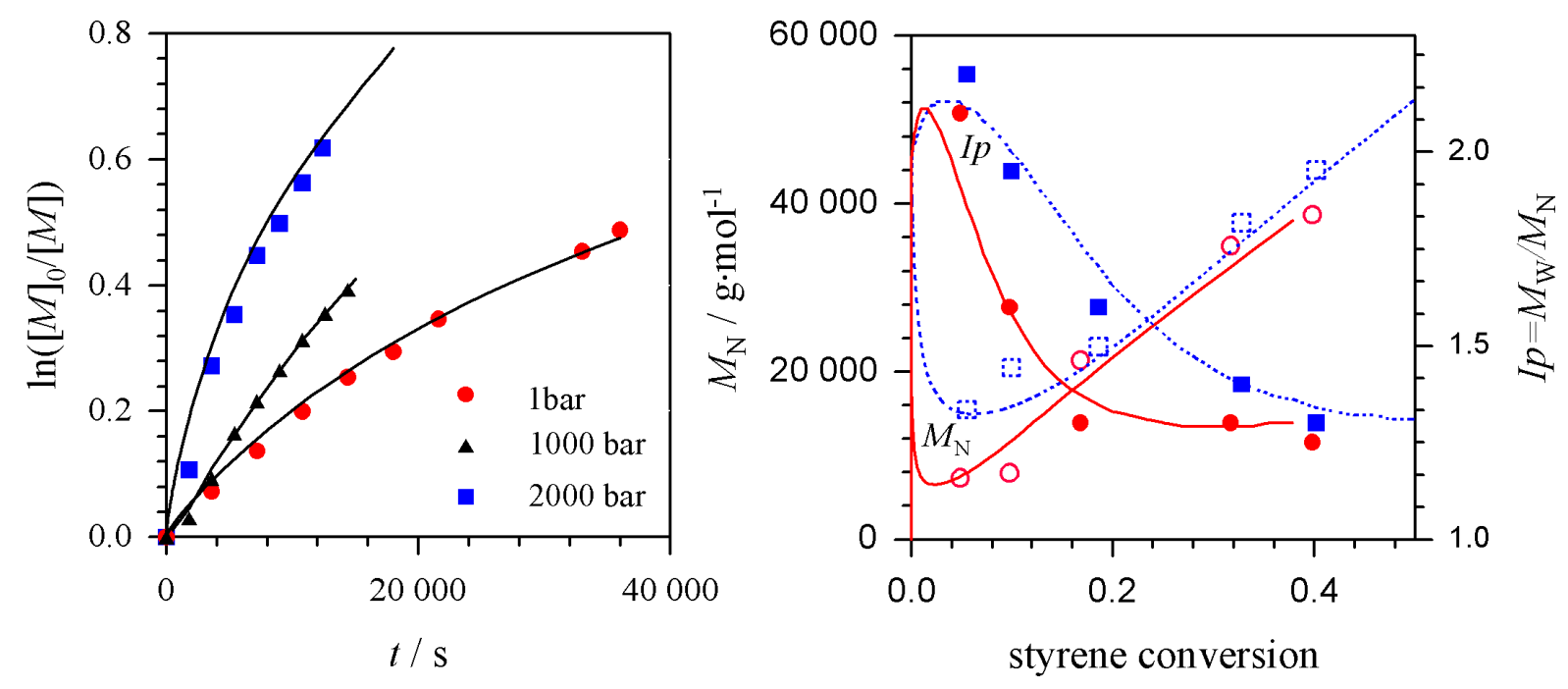

Fig. 6.13. Plot of $\ln ([M] d[M])$ vs time and of polydispersity (filled points) and molecular weight (open points) as a function of styrene conversion for polymerizations in the presence of $0.11 \mathrm{~mol} \%$ of styryl-TEMPO at $125^{\circ} \mathrm{C}$ and at pressures up to 2000 bar. The lines are from PREDICI ${ }^{\circledR}$ simulations, the data points are from experiments.

The estimated rate coefficient $k_{\mathrm{d}}$ at $125^{\circ} \mathrm{C}$ for the bond cleavage of the dormant species from TEMPO and the growing radical is one order of magnitude higher than the value given in the literature for the bond dissociation of styryl-TEMPO ${ }^{7-11,17} . k_{\mathrm{d}}$ is reported to be chain-length dependent and the value estimated in this work is close to the one determined by German et al. ${ }^{17}$ for the polymeric analog (approximately 75 units) by monitoring nitroxide concentration via ESR. The literature values are summarized in Table 6.14. The small difference at low monomer conversion between experimental and simulated data may be explained by the chain length dependence of $k_{\mathrm{d}}$, which is not considered in the model.

\begin{tabular}{ccccc}
\hline $\begin{array}{l}\text { conditions } \\
125^{\circ} \mathrm{C}\end{array}$ & $\begin{array}{c}k_{\mathrm{c}} \\
\mathrm{L} \cdot \mathrm{mol}^{-1} \cdot \mathrm{s}^{-1}\end{array}$ & $\begin{array}{c}k_{\mathrm{d}} \\
\mathrm{s}^{-1}\end{array}$ & $K=k_{\mathrm{d}} / k_{\mathrm{c}}$ & source \\
\hline \multicolumn{1}{c}{ styryl-TEMPO, 1 bar } & $2.5 \cdot 10^{8}$ & 2 to $5 \cdot 10^{-4}$ & 1 to $2 \cdot 10^{-12}$ & literature $^{7-11}$ \\
polystyryl-TEMPO1 bar & - & $3.2 \cdot 10^{-3}$ & - & literature $^{7-11}$ \\
1 bar & $9.0 \cdot 10^{5}$ & $1.0 \cdot 10^{-3}$ & $1.1 \cdot 10^{-9}$ & this work \\
2000 bar & $2.4 \cdot 10^{5}$ & $6.2 \cdot 10^{-4}$ & $2.6 \cdot 10^{-9}$ & this work \\
\hline
\end{tabular}

Table 6.14. Summary of fitted rate coefficients $k_{c}$ and $k_{d}$ for the alkoxyamine styryl-TEMPO at $125^{\circ} \mathrm{C}$ and comparison with literature values. 
The simulations performed for TEMPO-mediated polymerization at $115^{\circ} \mathrm{C}$ and 2000 bar are also presented in Figure 6.16. The rate coefficient of spontaneous thermal initiation of styrene at $115^{\circ} \mathrm{C}$ and 2000 bar was set to $k_{\mathrm{i}}=1.3 \cdot 10^{-9} \mathrm{~s}^{-1}$, so that simulations are in good agreement with the experimental data from spontaneous thermal polymerization. Temperature and pressure dependencies of $k_{\mathrm{c}}$ and $k_{\mathrm{d}}$ are taken as determined for styryl-TEMPO : $\Delta V^{\ddagger}\left(k_{\mathrm{c}}\right)$ $=22 \mathrm{~cm}^{3} \cdot \mathrm{mol}^{-1}, \Delta V^{\ddagger}\left(k_{\mathrm{d}}\right)=10 \mathrm{~cm}^{3} \cdot \mathrm{mol}^{-1}$. The coefficients for the reversible dissociation of styryl-TEMPO used in PREDICI ${ }^{\circledR}$ simulations are reported in Table 6.15. They allow for satisfactory description of styrene polymerization in the presence of styryl-TEMPO at low temperature.

\begin{tabular}{ccccc}
\hline conditions & $\begin{array}{c}k_{\mathrm{c}} \\
\mathrm{L} \cdot \mathrm{mol}^{-1} \cdot \mathrm{s}^{-1}\end{array}$ & $\begin{array}{c}k_{\mathrm{d}} \\
\mathrm{s}^{-1}\end{array}$ & $K=k_{\mathrm{d}} / k_{\mathrm{c}}$ & source \\
& $2.2 \cdot 10^{5}$ & $2.3 \cdot 10^{-4}$ & $1.0 \cdot 10^{-9}$ & this work \\
\hline $125^{\circ} \mathrm{C}, 2000$ bar & & & \\
\hline
\end{tabular}

Table 6.15. Summary of fitted rate coefficients $k_{c}$ and $k_{d}$ for the reversible dissociation of styryl-TEMPO at $115^{\circ} \mathrm{C}$ and 2000 bar.

Calculated molar mass distributions, polydispersity indices, and polymerization rates are very close to the experimental data. PREDICI ${ }^{\circledR}$ simulations represent the influence of the adduct concentration on polymerization rate and molar mass evolution as it was experimentally observed in Section 6.2.4. A lower alkoxyamine concentration induces a slight decrease of polymerization rate. The alkoxyamine concentration determines the molecular weight as theoretically predicted. A comparison of experimental and simulated MWDs for styrene polymerization in the presence of $0.11 \mathrm{~mol} \%$ styryl-TEMPO at 2000 bar and $115^{\circ} \mathrm{C}$ is reported in Fig. 6.16. The good agreement between experimental and simulated MWDs indicates that the rate coefficients $k_{\mathrm{c}}$ and $k_{\mathrm{d}}$ are reasonable. 


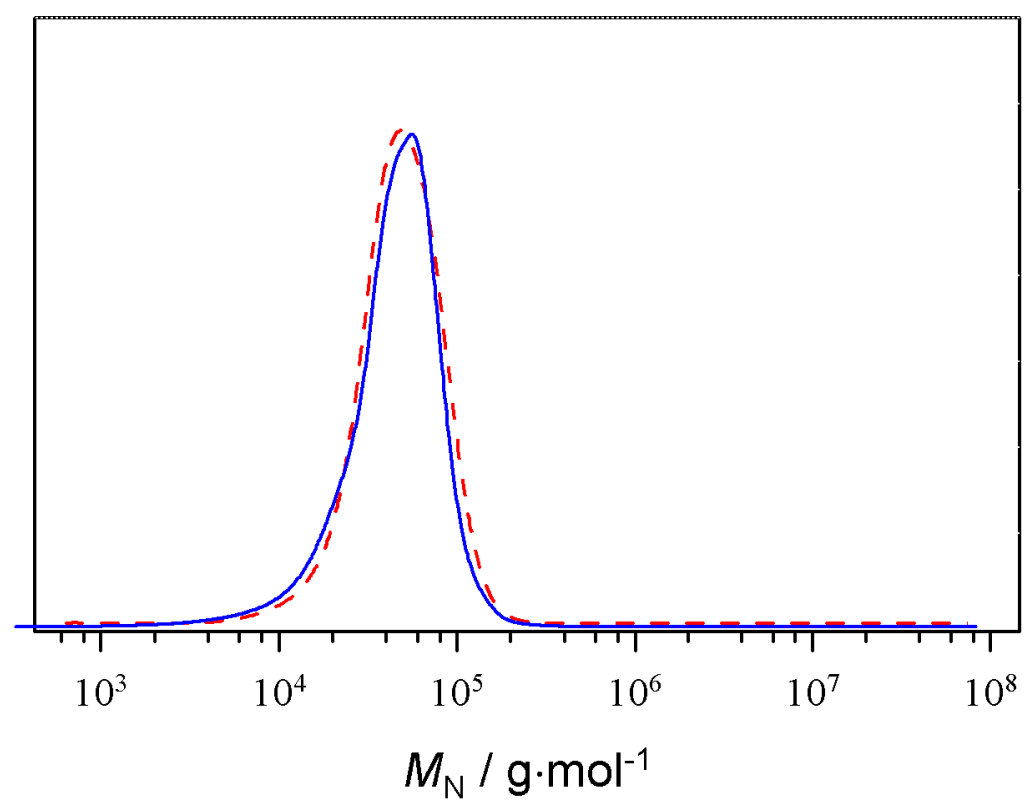

Fig. 6.16. Experimental (solid lines) and simulated (dashed lines) molecular weight distributions for styrene polymerization in the presence of $0.11 \mathrm{~mol} \%$ styrylTEMPO at 2000 bar and $115^{\circ} \mathrm{C}$. Data at approximately $50 \%$ conversion in 9 hours.

PREDICI $^{\circledR}$ simulations were also performed at lower temperatures in order to determine the temperature limit for controlled styrene polymerizations under pressure. The plot of molecular weight and polydispersity index as a function of monomer conversion for styryl-TEMPO-mediated styrene polymerizations at 2000 bar and at temperatures from $115^{\circ} \mathrm{C}$ up to $95^{\circ} \mathrm{C}$ are presented in Fig. 6.17. Simulations between $95^{\circ} \mathrm{C}$ and $115^{\circ} \mathrm{C}$ show that controlled styrene polymerization is possible under high pressure at these temperatures. Polydispersity indices lower than 1.5 are obtained above $30 \%$ conversion and molar masses increase with monomer conversion irrespective of the applied temperature. Polydispersity slightly increases with decreasing temperature but polydispersity indices at $50 \%$ conversion are close to 1.25 and 1.35 at 115 and $95^{\circ} \mathrm{C}$, respectively. Basically, controlled styrene polymerization is possible at $95^{\circ} \mathrm{C}$ but low temperature induces very low polymerization rate. Polymerization time to reach $50 \%$ conversion at 2000 bar is 7 hours at $115^{\circ} \mathrm{C}, 21$ hours at $105^{\circ} \mathrm{C}$ and approaches 40 hours at $95^{\circ} \mathrm{C}$.

These simulations show that mediator controlled styrene polymerization under high pressure may be carried out at reasonably low temperatures. High pressure presents a relevant advantage in that polymerization rate is increased. 


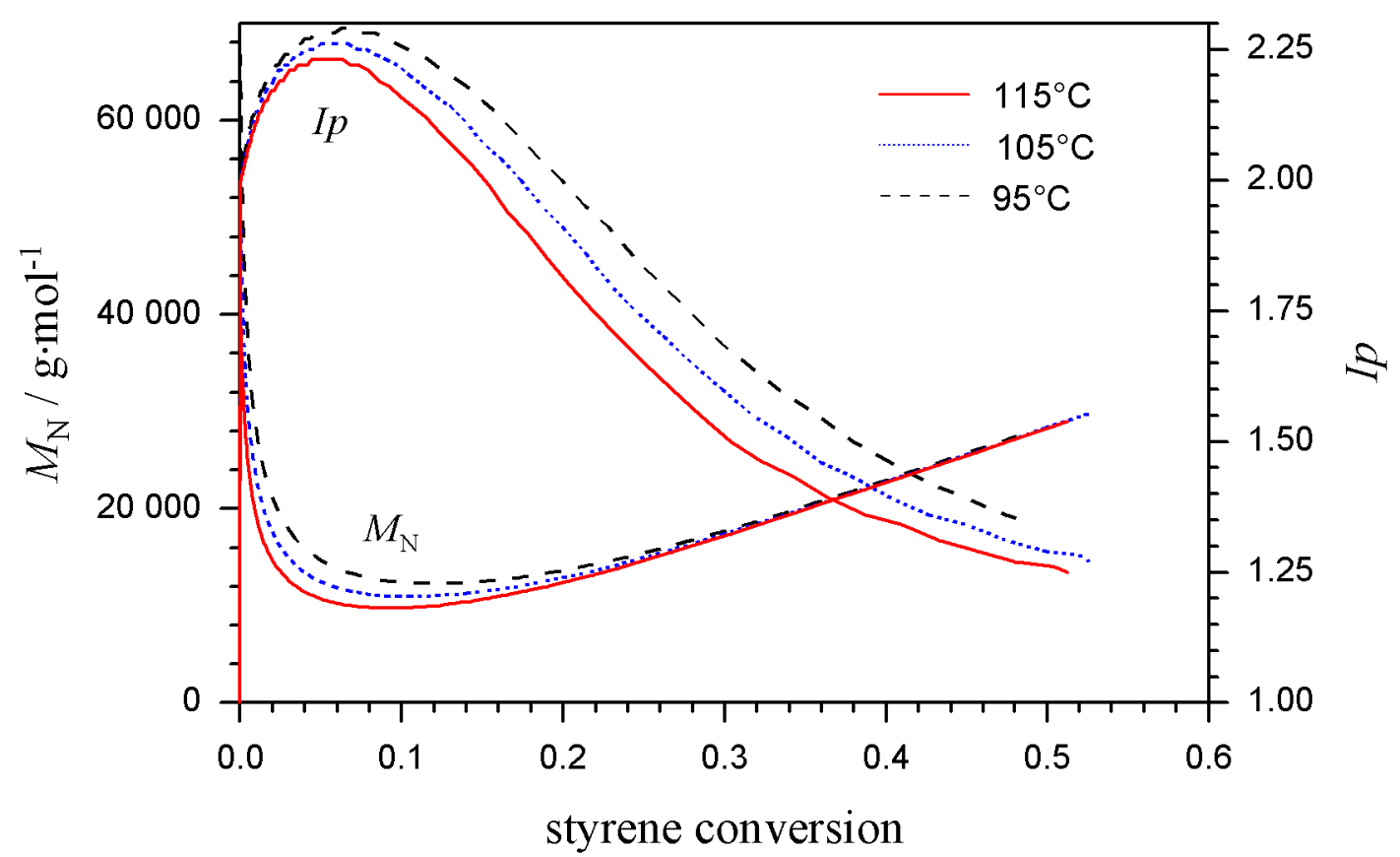

Fig. 6.17. $\quad$ PREDICI $^{\circledR}$ simulations of molecular weight and polydispersity index as a function of monomer conversion for styrene polymerizations in the presence of 0.220 mol\% styryl-TEMPO at 2000 bar between 115 and $95^{\circ} \mathrm{C}$.

\subsubsection{Simulation of SG1-mediated styrene CRP}

Rate coefficients $k_{\mathrm{c}}$ and $k_{\mathrm{d}}$ for styryl-SG1 and the polymeric equivalent (polystyrylSG1) at 1 bar and $120^{\circ} \mathrm{C}$ have already been reported by Tordo et al. ${ }^{15}$. They were adjusted by PREDICI $^{\circledR}$ simulations to satisfactorily describe the measurements at low and high pressure.

\begin{tabular}{ccccc}
\hline $120^{\circ} \mathrm{C}$ & $k_{\mathrm{c}}$ & $k_{\mathrm{d}}$ & $K$ & source \\
& $\mathrm{L} \cdot \mathrm{mol}^{-1} \cdot \mathrm{s}^{-1}$ & $\mathrm{~s}^{-1}$ & & \\
\hline polystyryl-SG1, 1 bar & $7 \cdot 10^{5}$ & $3 \cdot 10^{-3}$ to $10^{-2}$ & $4 \cdot 10^{-9}$ to $10^{-8}$ & 12 \\
1 bar & $7 \cdot 10^{5}$ & $2.7 \cdot 10^{-3}$ & $3.8 \cdot 10^{-9}$ & this work \\
2000 bar & $7 \cdot 10^{5}$ & $2.4 \cdot 10^{-3}$ & $3.4 \cdot 10^{-9}$ & this work \\
\hline
\end{tabular}

Table 6.18. Rate coefficients for the cleavage of the alkoxyamine styryl-SG1 at $120^{\circ} \mathrm{C}$ from PREDICI $^{\circledR}$ simulations 
PREDICI $^{\circledR}$ simulations allow for a good correlation of the experimental molecular weights and polydispersities at 1 and 2000 bar as shown in Figure 6.19. Polymerization rates reported in Section 6.2.5 are also correctly described. Fitted rate coefficients for the reversible dissociation of styryl-SG1 are presented in Table $6.18 . k_{\mathrm{c}}$ is set to $7 \cdot 10^{5} \mathrm{~L} \cdot \mathrm{mol}^{-1} \cdot \mathrm{s}^{-1}$ at $120^{\circ} \mathrm{C}$ and is considered pressure independent. $k_{\mathrm{d}}$ is set to $2.4 \cdot 10^{-3} \mathrm{~s}^{-1}$ and $2.7 \cdot 10^{-3} \mathrm{~s}^{-1}$ at $120^{\circ} \mathrm{C}$ and at 1 and 2000 bar, respectively. The obtained rate coefficients are in the range of values reported by Tordo et al. ${ }^{15}$ for the polymeric analogue. The rate coefficient for the dissociation of styryl-SG1 and for $K$ are found to be slightly pressure dependent.

In Fig 6.19, the simulated and experimental $M_{\mathrm{N}}$ and $I p$ values obtained as a function of styrene conversion are compared for S-SG1-mediated styrene polymerization at $120^{\circ} \mathrm{C}$ and 2000 bar. At high alkoxyamine concentration $(0.283 \mathrm{~mol} \%)$ the efficiency factor of styryl-SG1 was set to unity. Simulations performed via the program PREDICI ${ }^{\circledR}$ provide data close to the experimentaly observed values. In order to fit the polymerization data at low alkoxyamine concentration, the efficiency of the alkoxyamine was set to 0.7 . With this modification, the PREDICI $^{\circledR}$ calculations at 2000 bar are able to satisfactorily represent the experiments at low alkoxyamine concentration. The lower efficiency factor is due to termination processes in the early period of the polymerization.

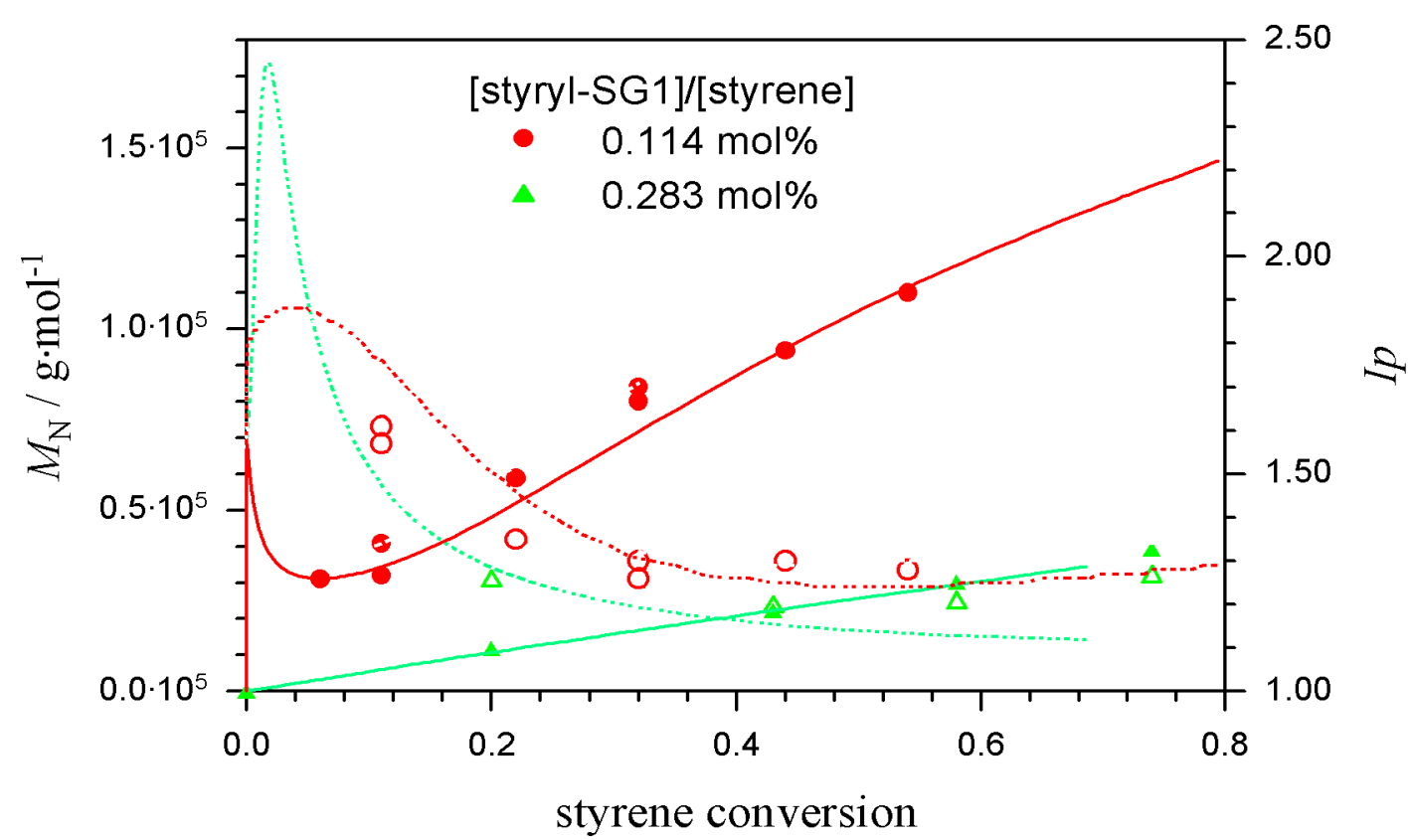

Fig. 6.19. Comparison of experimental and simulated (solid lines) molar mass (filled points) and polydispersity index (open points) evolutions for styrene polymerization at $120^{\circ} \mathrm{C}$ and 2000 bar with different concentrations of styryl$S G 1$. 
Simulations have also been carried out at 1 bar. Simulated $M_{\mathrm{N}}$ increases linearly with conversion up to about $80000 \mathrm{~g} \cdot \mathrm{mol}^{-1}$ which is in good agreement with experimental data (see Section 6.2.4). After $35 \%$ conversion, the PREDICI ${ }^{\circledR}$ simulations for ambient pressure gives no reasonable results.

Further simulations for SG1-mediated styrene polymerization have been performed at $120^{\circ} \mathrm{C}$ and 2000 bar by introducing a $10 \mathrm{~mol} \%$ excess of nitroxide in the system in order to improve the molecular weight control in the early stage of the polymerization. Calculated molecular weights and polydispersities are plotted in Fig. 6.20. An excess of nitroxide is favorable only in the very beginning of the reaction by lowering $M_{\mathrm{N}}$ and $I p$. In fact, the control of the polymerization seems to be effective even in the early period of the polymerization. The molar mass starts to increase with monomer conversion already at very low conversion (the extrapolation to $0 \%$ conversion yields $M_{\mathrm{N}}=8000 \mathrm{~g} \cdot \mathrm{mol}^{-1}$ ) and the polydispersities are lower. $I p=1.5$ is reached after $16 \%$ conversion compared to $25 \%$ without excess nitroxide. Above $30 \%$ conversion, the influence of nitroxide excess on the $M_{\mathrm{N}}$ and $I p$ evolution is negligible.

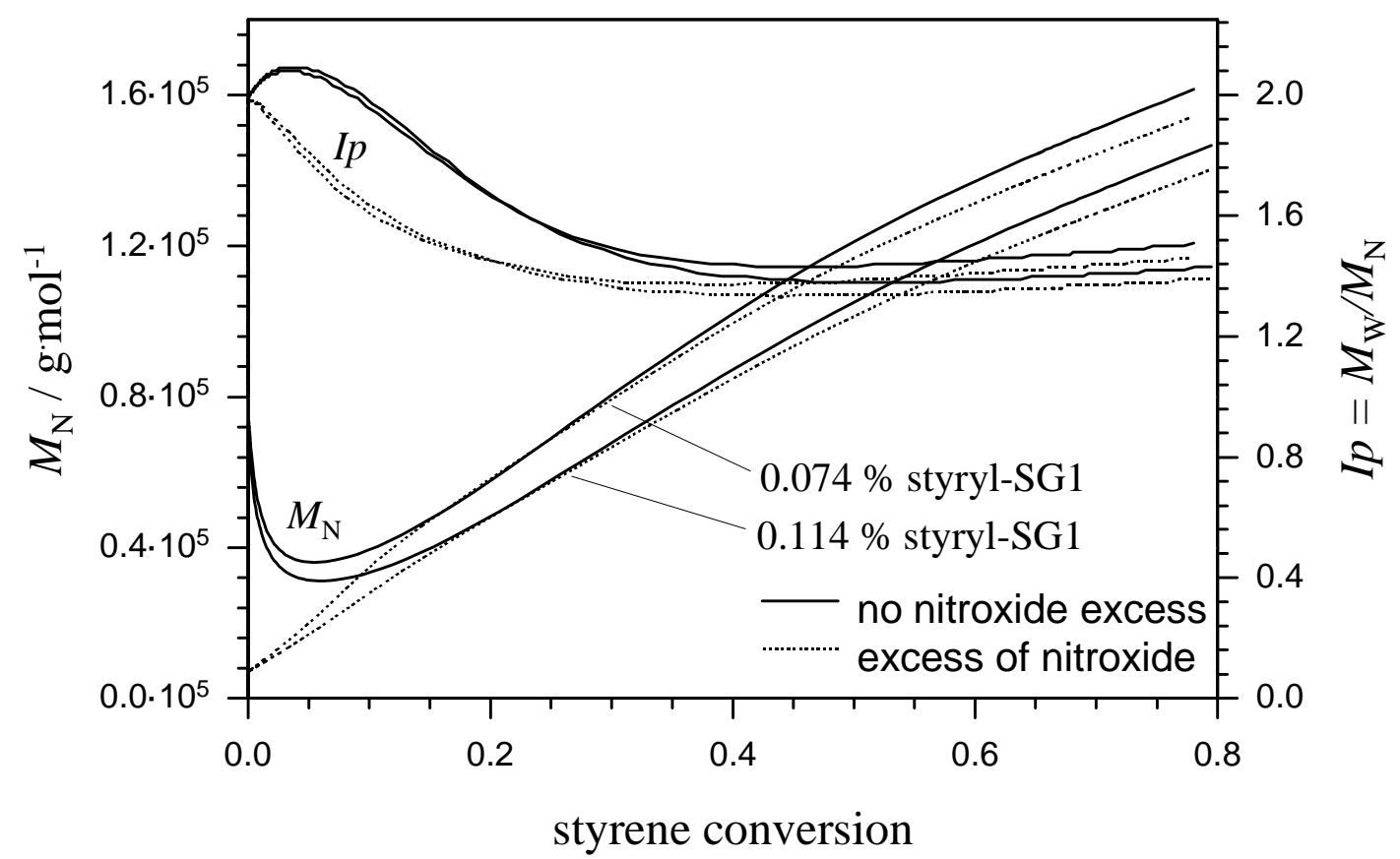

Fig. 6.20. Influence of nitroxide excess on molar mass and polydispersity index evolutions for styrene polymerizations at $120^{\circ} \mathrm{C}$ and 2000 bar in the presence of different concentrations of styryl-SG1 (all data are from PREDICI ${ }^{\circledR}$ simulations). 
Dead polymer and dormant species concentrations during styrene polymerizations have also been calculated. In Fig. 6.21 these concentrations are given as a function of monomer conversion for styryl-SG1-mediated polymerization with or without nitroxide excess. The dead polymer concentration increases during the polymerization because of self initiation, transfer and termination reaction. The dormant species concentration rapidly reaches a maximum at approximately $18 \%$ conversion (during this period the control is not very effective) and then slowly decreases because of transfer and termination reactions. Dead polymer concentration is reported to be not influenced by the nitroxyl radical excess. A nitroxide excess induces an increase of about $15 \%$ of the dormant species concentration and the maximal concentration is reached faster. With an excess of nitroxide the persistent radical effect is reached earlier which is fully consistent with the treatment presented by Fischer (see Section 3.5).

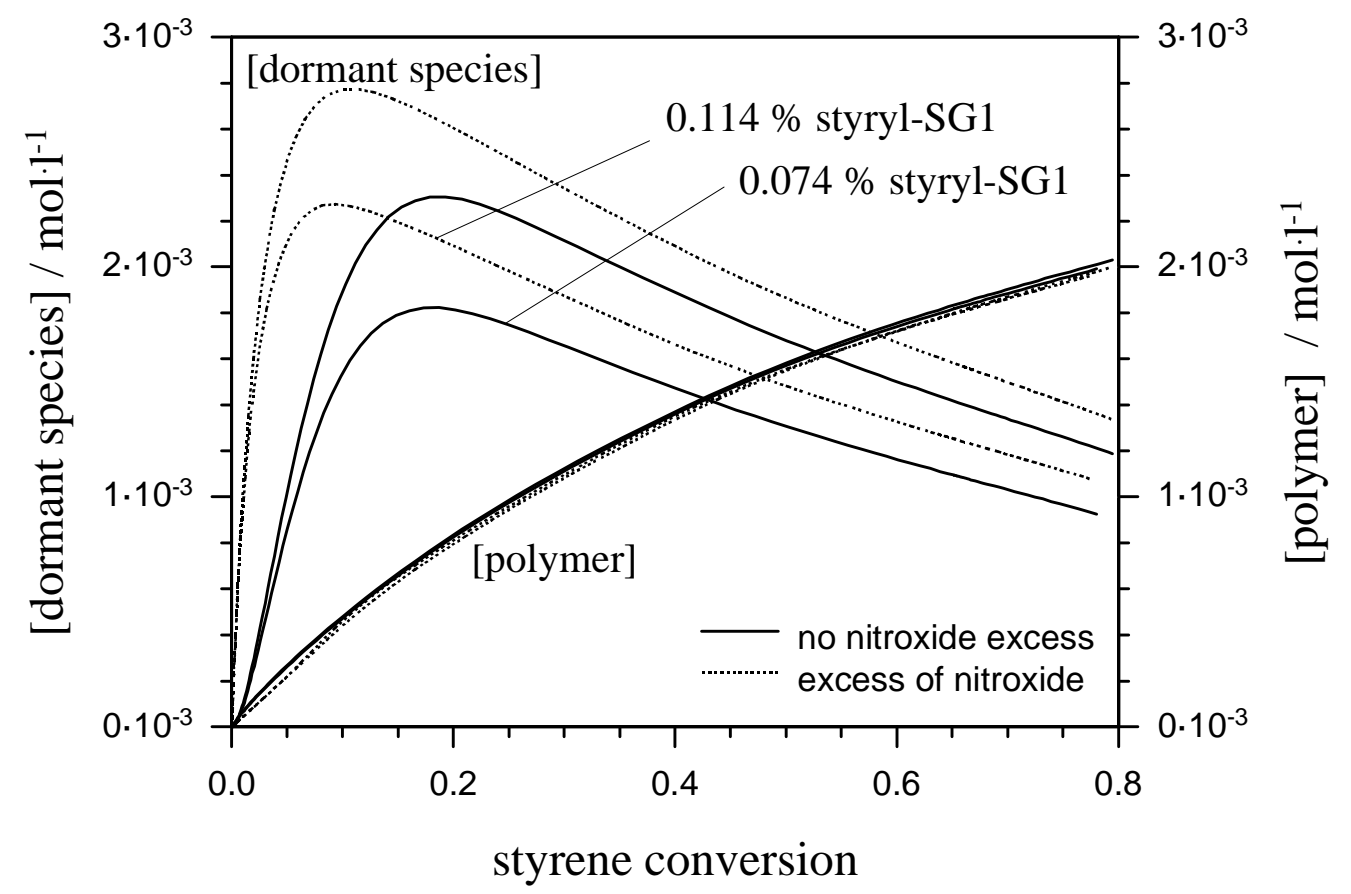

Fig. 6.21. Influence of nitroxide excess on dormant species and on dead polymer concentration for styrene polymerizations at $120^{\circ} \mathrm{C}$ and 2000 bar in the presence of different concentrations of styryl-SG1 (all data are from PREDICI ${ }^{\circledR}$ simulations).

Fig. 6.21 also shows the effect of lower alkoxyamine concentration on the dormant species and on dead polymer concentration. A decrease of $0.114 \mathrm{~mol} \%$ to $0.074 \mathrm{~mol} \%$ of styryl-SG1 induces a decrease of $25 \%$ of concentration of dormant species. It is assumed that 
the dead polymer concentration seems to be not dependent on the alkoxyamine concentration, the influence of dead polymer will be clearer and the polydispersity index will be higher at low alkoxyamine concentration. Equal amounts of dormant species and dead polymer occur at 43 and $52 \%$ for styrene polymerization with 0.074 and 0.114 mol\% styryl-SG1, respectively.

The results in Figs 6.20 and 6.21 show that polystyrene from reaction at low initiator concentration at conversion above $50 \%$ may be controlled (showing narrow polydispersity) but to a major part is not living.

\subsection{Nitroxide-mediated (meth)acrylate polymerization}

\subsubsection{Nitroxide-mediated butyl acrylate polymerization}

The experimental results presented in Section 5.4.1, showed the influence of pressure on polymerization rate. Firstly, it was observed, as in the case of styrene CRP, that high pressure does not prevent the control of styrene polymerization. As it was expected, high pressure induces an enhancement of butyl acrylate polymerization rate with SG1. The increase in polymerization rate is due to the influence of high pressure on $k_{\mathrm{p}}$ and $k_{\mathrm{t}}$, which for conventional styrene polymerization was already shown by Buback et al. ${ }^{16,17}$. The pressure influence on activation and on deactivation rate coefficients of the growing radical, $k_{\mathrm{c}}$ and $k_{\mathrm{d}}$, has not been yet investigated. It appears that high pressure does not improve the control of the polymerization, in particular not at low BA conversion.

Experimental molar masses were found to be higher than the values predicted from the alkoxyamine concentrations in BA due to the fact that the persistent radical effect does not work in the early period of the polymerization. It is assumed that high alkoxyamine concentration induces a lowering of the molecular weight and a relevant narrowing of the MWD, as it was observed in Section 6.2.4. for styrene CRP.

The limitation of molecular weight toward high values was already observed for styrene polymerization at low SG1 concentration. It was shown to be due to the effect of dead polymer on polydispersity. The accumulation of radicals from spontaneous initiation, due to high polymerization time, combined with the irreversible deactivation of the dormant species 
during the polymerization, induces a decrease of persistent radical concentration, and thus an increase of the concentration of dead polymer and a broadening of the MWD.

\subsubsection{Nitroxide-mediated methyl methacrylate polymerization}

During recent years, several authors demonstrated the capacity of nitroxides to polymerize a wide variety of monomers and suggested that controlled radical polymerization should be feasible with monomers having carboxylic, epoxy functional groups... This feature was probed by copolymerizing mixtures of styrene, or butyl acrylate, with a variety of reactive monomers. Control was maintained even in the presence of a significant amount of the reactive monomer. On the other hand, nitroxides which were able to control acrylate polymerizations, failed to control methacrylate homopolymerizations ${ }^{18}$. The ability of this type of nitroxides to control acrylate homopolymerization suggested that this system may be applicable to a wide selection of monomers, similar to ATRP procedures. Well-defined poly (methyl) methacrylates have been prepared by ATRP within the molecular weight range from 1000 to $180000 \mathrm{~g} \cdot \mathrm{mol}^{-1}$. In the region from 1000 to $90000 \mathrm{~g} \cdot \mathrm{mol}^{-1}$, polydispersities are less than 1.1. For a typical bromide-mediated MMA ATRP in solution, the estimated constant ${ }^{19}$, $K=2 \cdot 10^{-7}$, is larger than in styrene polymerization despite the lower temperature used. A typical ATRP system consists of a monomer, an initiator, and a metal complexed with ligands. Polymerization systems utilizing this concept have been developed using $\mathrm{Cu}(\mathrm{I}){ }^{20-24}$, $\mathrm{Ni}(\mathrm{II}){ }^{25,26}, \mathrm{Ru}(\mathrm{II}) / \mathrm{Al}(\mathrm{OR}) 3^{27}$, and $\mathrm{Fe}$ (II) ${ }^{28-30}$ complexes and many different complexes using various initiation systems ${ }^{31-52}$.

Methyl methacrylate homopolymerization in the presence of nitroxide, however, did not allow for the preparation of PMMA with polydispersities less than 1.5. Recently, Rizzardo et al. reported a comparison of a series of nitroxides ${ }^{53}$, including the imidazolidinone nitroxides, in controlling MMA polymerization at $90^{\circ} \mathrm{C}$. Narrow polydispersities in the range from 1.4 to 1.7 were obtained with the five-membered ring nitroxides. Although these polydispersities are not exceptional, as compared with the very narrow polydispersities obtained in styrene polymerizations, the results compare very favorably with the values in the range from 3.1 to 4.1 obtained with six-membered ring nitroxide (TEMPO and derivatives) or open-chain nitroxides (tert-amyl-tert-butyl nitroxide). Best monomer conversions (up to $38 \%$ ) were obtained with imidazolidinone nitroxide. All other nitroxides gave conversions 
below $20 \%$. NMR analyses showed that a methyl methacrylate macromonomer is formed by disproportionation of the MMA propagating species with nitroxide. After a short inhibition period due to the nitroxide excess, little propagation occurs and the propagating radical irreversibly disproportionates with nitroxide to give a hydroxylamine. The results of simulations from Rizzardo et al. ${ }^{54}$ support this hypothesis.

In order to improve methyl methacrylate controlled polymerization, it was decided to carry out some polymerizations under high pressure in the presence of nitroxides which differ in reactivity. High pressure may have an influence on disproportionation of the MMA propagating species and on the combination of nitroxide and propagating radical. MMA polymerizations were performed in the presence of SG1, an open chain nitroxide which is both reactive and capable of controlling acrylate polymerization, and a five-membered ring nitroxide, DPAIO, which is very stable and of lower reactivity. PREDICI ${ }^{\circledR}$ simulations will $^{2}$ allow an estimation of the disproportionation rate coefficient at high pressure.

MMA polymerization, as could be seen from the result presented in Section 5.4.2, is not controlled by either the styryl-SG1 nor the styryl-DPAIO alkoxyamine. SG1-mediated MMA polymerization is much slower than the conventional FRP of MMA. Styryl-SG1 leads to high monomer conversion, but also to very high molecular weights. High pressure induces a strong increase of polymerization rate and of number average molecular weight for SG1mediated MMA polymerization. The $M_{\mathrm{N}}$ values are 125000 and $280000 \mathrm{~g} \cdot \mathrm{mol}^{-1}$ at 50 and 2000 bar, respectively. At 1 bar, the plots of $\ln \left([M] /[M]_{0}\right)$ vs time exhibit a strong downward curvature from $30 \%$ conversion (see Fig. 5.21), due to termination processes. At 2000 bar, termination reactions are less important and the $\ln \left([M] /[M]_{0}\right)$ vs time plot only exhibits a slight downward curvature and leads to high conversion. For SG1-mediated MMA polymerization, the high dissociation rate of the dormant species, which is associated with the high bulkiness of SG1, probably allows for a rapid growth which leads to the high molecular weight observed. This propagating radical may finally undergo termination, transfer to monomer or may disproportionate with nitroxide to yield dead polymer. The use of SG1 as the mediator for MMA polymerization does not lead to a controlled MMA polymerization but induces a significant decrease of termination and transfer processes, leading to $M_{\mathrm{N}}$ values which are much higher than with AIBN-initiated polymerization. 
Molecular weights observed for DPAIO-mediated MMA polymerization at $95^{\circ} \mathrm{C}$ are much lower than in the case of AIBN-initiated methyl methacrylate polymerization. Polymerization rates are also much lower, even much lower than for SG1-mediated MMA polymerization. The dormant species seems to be very stable and yields a low release of growing radicals and thus low propagation rates. DPAIO nitroxide seems to play the role of a chain-transfer agent that induces low molecular weights. The dormant species may undergo a low dismutation reaction (see Scheme 6.22), as it was proposed by Rizzardo et al ${ }^{53}$.

Although the five-membered nitroxide should to be favorable compared to open chain nitroxides, the reactivity of the nitroxide is too low as to allow for a good control. The dependence of the potential to control polymerization on the type of nitroxide can be attributed to the relative importance of combination vs disproportionation reactions as was proposed by Rizzardo et al ${ }^{53}$.
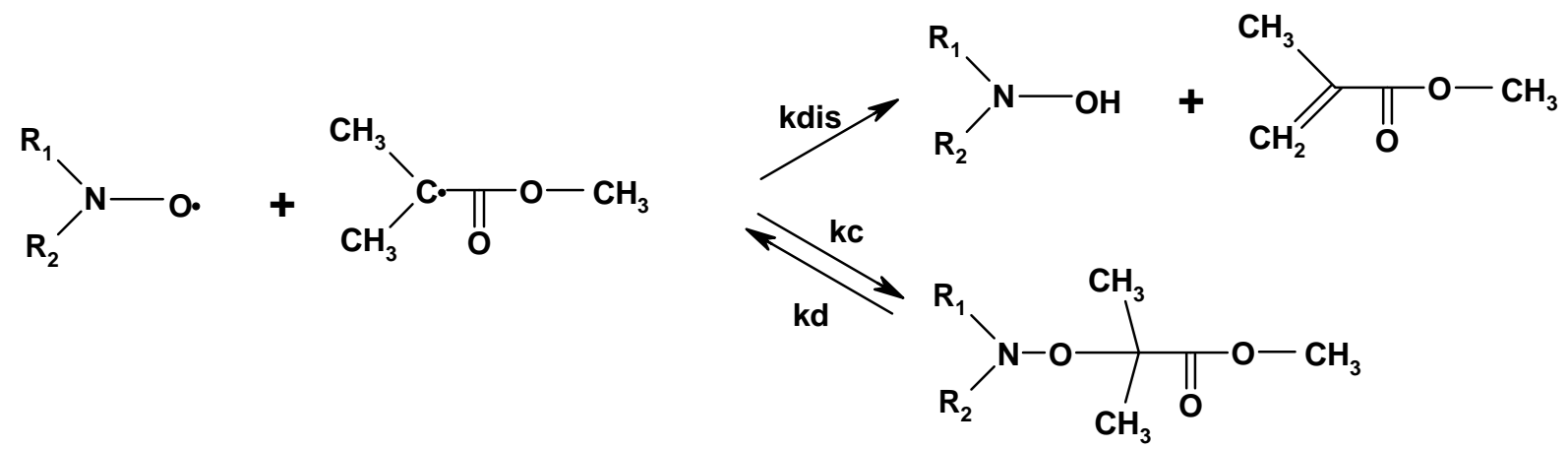

Scheme 6.22. Competition between combination and disproportionation during nitroxidemediated FRP of methyl methacrylate.

The competition between recombination and disproportionation (presented in Scheme 2) during free-radical polymerization in the presence of nitroxide has been estimated by Tordo et al. ${ }^{55}$. The results for the difference in reaction enthalpies are presented in Table 6.23. 


\begin{tabular}{ccc}
\hline & $\Delta \Delta H\left(\mathrm{kcal} \cdot \mathrm{mol}^{-1}\right)=\Delta H_{\mathrm{c}}-\Delta H_{\mathrm{dis}}$ \\
& TEMPO & $\mathrm{SG} 1$ \\
\hline styrene & -8.6 & -5.8 \\
methyl acrylate & -3.6 & -2.5 \\
methyl methacrylate & 2.1 & 6.8 \\
\hline
\end{tabular}

Table 6.23. Difference in reaction enthalpies for the combination and disproportionation reactions of TEMPO and SG1 with several monomer molecules at $90^{\circ} \mathrm{C}$ and 1 bar.

The positive value of $\Delta \Delta H$ determined for MMA polymerization in the presence of TEMPO or SG1 shows that hydrogen abstraction is favored. In these cases at 1 bar, neither TEMPO, nor SG1 are able to control MMA polymerization. Considering the results from Section 5.4.2. and especially the large polydispersities at 2000 bar shows that high pressure does not reverse the sign of $\Delta \Delta H$ for MMA polymerization.

For a better understanding of the influence of the nitroxide, the evaluation ${ }^{55}$ of the pre-cited competition between combination and disproportionation during polymerization of MMA for different nitroxides is presented in Table 6.24. The $\Delta \Delta H$ value may be correlated with the steric crowding in the vicinity of the oxygen atom of the nitroxyl group.

\begin{tabular}{cccc}
\hline nitroxide & $\begin{array}{c}\mathrm{S} \\
\AA^{2}\end{array}$ & $\begin{array}{c}k_{\mathrm{c}} \cdot 10^{-7} \\
(\text { for benzyl radical) }\end{array}$ & $\begin{array}{c}\Delta \Delta H_{\text {MMA }} \\
\mathrm{kcal} \cdot \mathrm{mol}^{-1}\end{array}$ \\
\hline ABNO & 44.7 & $118 \pm 9$ & 0.5 \\
TMIO & 28.9 & $55 \pm 5$ & -0.6 \\
DPAIO & 27.0 & - & -0.2 \\
TEMPO & 23.7 & $48 \pm 8$ & 2.1 \\
DTBN & 21.2 & $46 \pm 4$ & - \\
SG1 & 8.0 & - & 6.8 \\
\hline
\end{tabular}

Table 6.24. Correlation between steric crowding around the oxygen atom in the nitroxide and the competition between recombination and disproportionation during MMA polymerization in the presence of nitroxides at $90^{\circ} \mathrm{C}$ and 1 bar ${ }^{55}$. 
where $\mathrm{S}$ is the accessible surface of a solvent molecule at the oxygen atom of the nitroxide. This surface which represents a measure of the steric crowding (see Scheme 6.25) of the nitroxide shows a good correlation with combination rate coefficients $k_{\mathrm{c}}$. The smaller the surface is (as with the bulky nitroxide SG1), the lower is the combination and the higher is the disproportionation of the growing radical. Contrary to the favorable predictions (negative $\Delta \Delta \mathrm{H})$ from theoretical calculations for MMA polymerization with DPAIO, the experiments did not show any sign of control of MMA polymerization.

In order to determinate the disproportionation rate coefficient with DPAIO in MMA polymerization, PREDICI ${ }^{\circledR}$ simulations have been carried out. MMA polymerization in the presence of SG1 was not simulated because of the unfavorable theoretical predictions presented in Table 6.24.

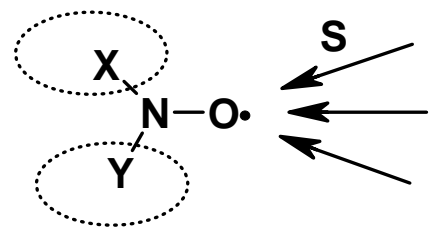

Scheme 6.25. Schematic representation of the accessible surface $S$ for a solvent molecule to the nitroxyl radical.

The main problem associated with kinetic simulations is the suitable selection of the rate coefficients. This is a particular problem in case of alkoxyamine-mediated polymerization where only very few rate constants have been measured directly. Fortunately, reliable rate coefficients have been determined in Prof. Tordo's laboratory. They allow to model MMA polymerization in the presence of alkoxyamines.

From previous work ${ }^{56,57}$ into MMA radical polymerization under high pressure, the rate coefficients relative to propagation and termination in MMA polymerization are available. It is assumed that all steps are chain length independent. The reactions which are taken into account are presented in Scheme 6.26. 
$\mathrm{R}_{\mathrm{n}}+\mathrm{M} \stackrel{k_{\mathrm{p}}}{\longrightarrow} \mathrm{R}_{\mathrm{n}+1}$

$\mathrm{R}_{\mathrm{n}}+\mathrm{R}_{\mathrm{m}} \stackrel{k_{\mathrm{tc}}}{\longrightarrow} \mathrm{P}_{\mathrm{n}+\mathrm{m}}$

$\mathrm{R}_{\mathrm{n}}+\mathrm{R}_{\mathrm{m}} \stackrel{k_{\mathrm{td}}}{\longrightarrow} \mathrm{P}_{\mathrm{n}}+\mathrm{P}_{\mathrm{m}}$

$\mathrm{R}_{\mathrm{n}}+\mathrm{M} \stackrel{k_{\mathrm{trM}}}{\longrightarrow} \mathrm{P}_{\mathrm{n}}+\mathrm{R}_{1}$

$\mathrm{R}_{\mathrm{n}}+\mathrm{N} \stackrel{k_{\mathrm{c}}}{\longrightarrow} \mathrm{R}_{\mathrm{n}} \mathrm{N}$

$\mathrm{R}_{\mathrm{n}} \mathrm{N} \stackrel{k_{\mathrm{d}}}{\longrightarrow} \mathrm{R}_{\mathrm{n}}+\mathrm{N}$

$\mathrm{R}_{\mathrm{n}}+\mathrm{N} \stackrel{k_{\text {dis }}}{\longrightarrow} \mathrm{P}_{\mathrm{n}}+\mathrm{NH}$

$\mathrm{R}_{\mathrm{n}}+\mathrm{NH} \stackrel{k_{\text {dis }}}{\longrightarrow} \mathrm{P}_{\mathrm{n}}+\mathrm{N}$ propagation

termination

transfer to monomer

reversible deactivation of

the propagating radical

disproportionation of the growing radical

with nitroxide

Scheme 6.26. Elementary reactions used in the simulation of nitroxide-mediated MMA homopolymerizations.

The conventional MMA FRP initiated by AIBN has been simulated and coefficients have been fitted to experimental data. $k_{\mathrm{p}}$ and $k_{\mathrm{t}}$ are taken from Buback et al. ${ }^{16,17}$. A comparison of polymerization rates and MWDs with simulated data for conventional MMA FRP is presented in Fig. 6.27. The perfect correlation between experimental and simulated data shows the quality of the rate coefficients used. 


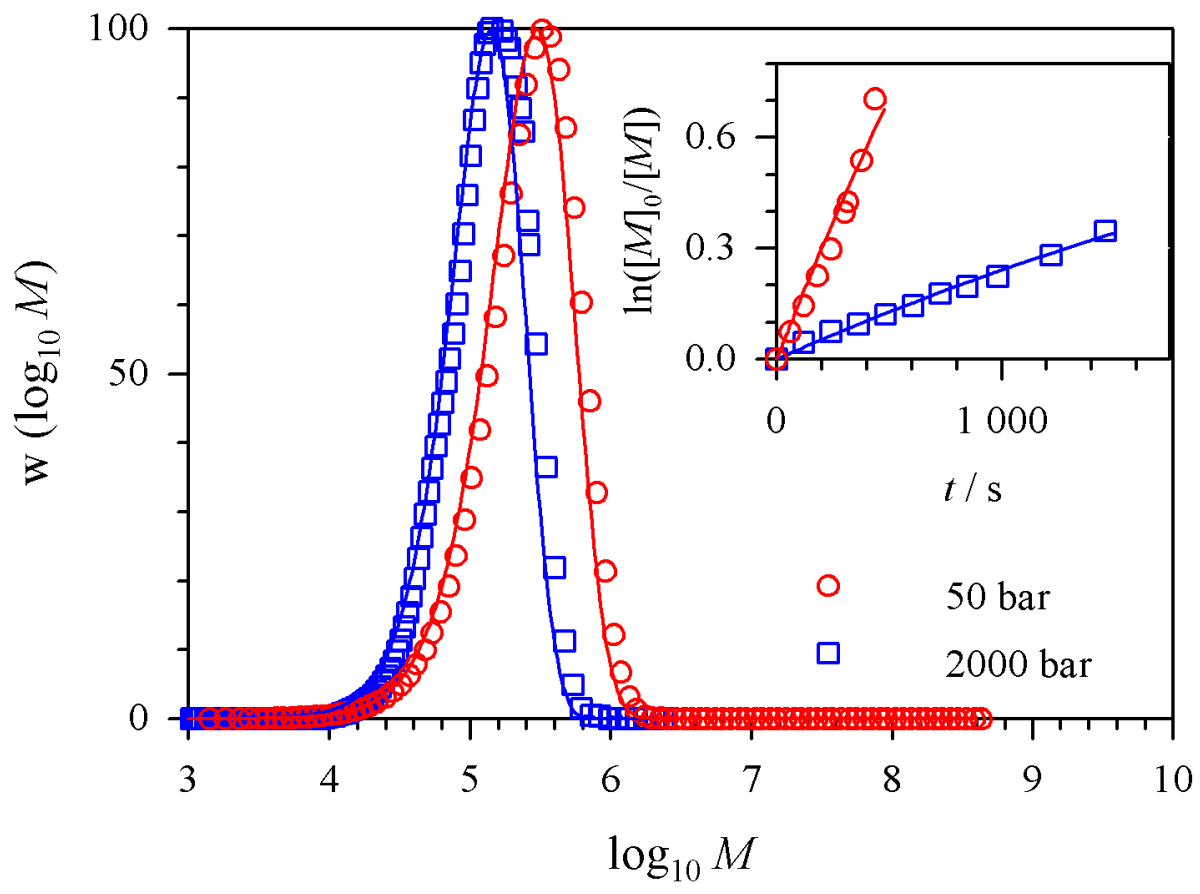

Fig. 6.27. Comparison of experimental (points) with simulated (lines) MWDs and polydispersity index vs time plots in insert for MMA polymerizations initiated by $A I B N$ at $95^{\circ} \mathrm{C}$ and at pressures up to 2000 bar. [AIBN]/[MMA] $=0.05 \mathrm{~mol} \%$, $T=95^{\circ} \mathrm{C}, k_{i A I B N} / s^{-1}=2 \cdot 10^{14} \exp (-14900 /(T / K))^{59}$.

From the known $\mathrm{C}-\mathrm{O}$ bond dissociation energies of styryl-TEMPO and MMATEMPO alkoxyamines which are rather similar, 15.7 and $14.9 \mathrm{kcal} \cdot \mathrm{mol}^{-1}{ }^{55}$, respectively, it has been assumed that $k_{\mathrm{d}}$ at $95^{\circ} \mathrm{C}$ for MMA-TEMPO should be close to $8.2 \cdot 10^{-5} \mathrm{~s}^{-1}$, the value for styryl-TEMPO ${ }^{60}$ at $95^{\circ} \mathrm{C}$.

The rate constant for combination of TEMPO with a styryl radical has been reported to be $7.6 \cdot 10^{5} \mathrm{~L} \cdot \mathrm{mol}^{-1} \cdot \mathrm{s}^{-1} 60$. The activation energy for this reaction is very small. A value for $k_{\mathrm{c}}$ at $95^{\circ} \mathrm{C}$ in the range $10^{5}-10^{6} \mathrm{~L} \cdot \mathrm{mol}^{-1} \cdot \mathrm{s}^{-1}$ seems to be reasonable.

The resulting data from simulation of polymerization rate, molecular weight and molecular weight distribution is reported in Fig. 6.29. The kinetic coefficients are given in Table 6.28. The rate coefficients implemented into the model allow for a good correlation between experimental and simulated polymerization rates and molecular weights at different pressures and also the simulated MWDs at 50 and 2000 bar agree with the experimental ones (see Fig. 6.29). 


\begin{tabular}{ccccc}
\hline rate constant & & 1 bar & 2000 bar & source \\
\hline$k_{\mathrm{p}}$ & $\mathrm{L} \cdot \mathrm{mol}^{-1} \cdot \mathrm{s}^{-1}$ & 1745 & 5085 & 56 \\
$k_{\mathrm{t}}$ & $\mathrm{L} \cdot \mathrm{mol}^{-1} \cdot \mathrm{s}^{-1}$ & $1.8 \cdot 10^{-8}$ & $4.5 \cdot 10^{-7}$ & 57 \\
$k_{\mathrm{tc}} / k_{\mathrm{td}}$ & & 0.3 & 0.3 & 54 \\
$k_{\mathrm{tr}}$ & $\mathrm{L} \cdot \mathrm{mol}^{-1} \cdot \mathrm{s}^{-1}$ & 0.15 & 0.15 & 58 \\
$k_{\mathrm{d}}$ & $\mathrm{s}^{-1}$ & $1.0 \cdot 10^{-4}$ & $1.0 \cdot 10^{-4}$ & this work \\
$k_{\mathrm{c}}$ & $\mathrm{L} \cdot \mathrm{mol}^{-1} \cdot \mathrm{s}^{-1}$ & $2.5 \cdot 10^{5}$ & $2.5 \cdot 10^{5}$ & this work \\
$k_{\mathrm{dis}}$ & $\mathrm{L} \cdot \mathrm{mol}^{-1} \cdot \mathrm{s}^{-1}$ & $5 \cdot 10^{-3}$ & $5.0 \cdot 10^{-3}$ & this work \\
\hline
\end{tabular}

Table 6.28. Rate coefficients used in DPAIO-mediated MMA simulations at $95^{\circ} \mathrm{C}$.

It should be noted that the kinetic parameters $k_{\mathrm{d}}$ and $k_{\mathrm{c}}$ obtained by fitting the experimental data are possibly not unique. The obtained $k_{\mathrm{d}}$ and $k_{\mathrm{c}}$ values are much smaller (by a factor of 100) than the data determined by Rizzardo et al. ${ }^{54}$ for MMA-TMIO, a fivemembered ring nitroxide. However if $k_{\mathrm{d}}$ and $k_{\mathrm{c}}$ for the system DPAIO-MMA are not in the order of magnitude as determined here, the simulated MWD does not still agree with experimental one. This is also true if higher $k_{\mathrm{d}}$ and $k_{\mathrm{c}}$ values with $K=k_{\mathrm{d}} / k_{\mathrm{c}}$ remaining constant, are implemented into the model. The equilibrium constant $K$ and disproportionation-combination ratio $\left(k_{\mathrm{dis}} / k_{\mathrm{c}}\right)$ have been demonstrated by Rizzardo et al. ${ }^{54}$ to be crucial parameters for the nitroxide-mediated MMA polymerization. In the case of DPAIO-mediated MMA polymerization, the disproportionation rate coefficient was set to $5 \cdot 10^{-3} \mathrm{~L} \cdot \mathrm{mol}^{-1} \cdot \mathrm{s}^{-1}$. Due to the low disproportionation rate of growing radical with DPAIO, the ratio $k_{\mathrm{dis}} / k_{\mathrm{c}}$ is very small, close to $10^{-8}$, which is by 3 to 5 orders of magnitude below the ratios determined by Rizzardo et al. ${ }^{54}$ for MMA polymerization with TMIO. This is unexpected but the values of Rizzardo et al. for the TMIO-MMA system do not allow to represent the molecular weights obtained in the experiments with the nitroxide DPAIO.

High conversions are reached under high pressure for DPAIO-mediated MMA polymerization due to combination of three phenomena $: k_{\mathrm{p}}$ increases and $k_{\mathrm{t}}$ decreases with pressure. Low disproportionation rate of the growing radical and a low alkoxyamine dissociation rate are expected. The simulations of experimental data indicates that the rate coefficients $k_{\mathrm{dis}}, k_{\mathrm{d}}$ and $k_{\mathrm{c}}$ (or more precisely the ratio $K=k_{\mathrm{d}} / k_{\mathrm{c}}$ ) are pressure independent. Thus high pressure will not induce a good control of the polymerization. The observed high $M_{\mathrm{N}}$ and high monomer conversion show that disproportionation of the growing radical with nitroxide does not play an important role, which is in agreement with the $\Delta \Delta H$ value for the 
system DPAIO-MMA at $90^{\circ} \mathrm{C}$ and 1 bar determined by Tordo et al. ${ }^{55}$. The simulations suggest that the uncontrolled polymerization is probably due to low $k_{\mathrm{d}}$ and $k_{\mathrm{c}}$ values, in particular due to a very low dissociation rate coefficient of the dormant species.
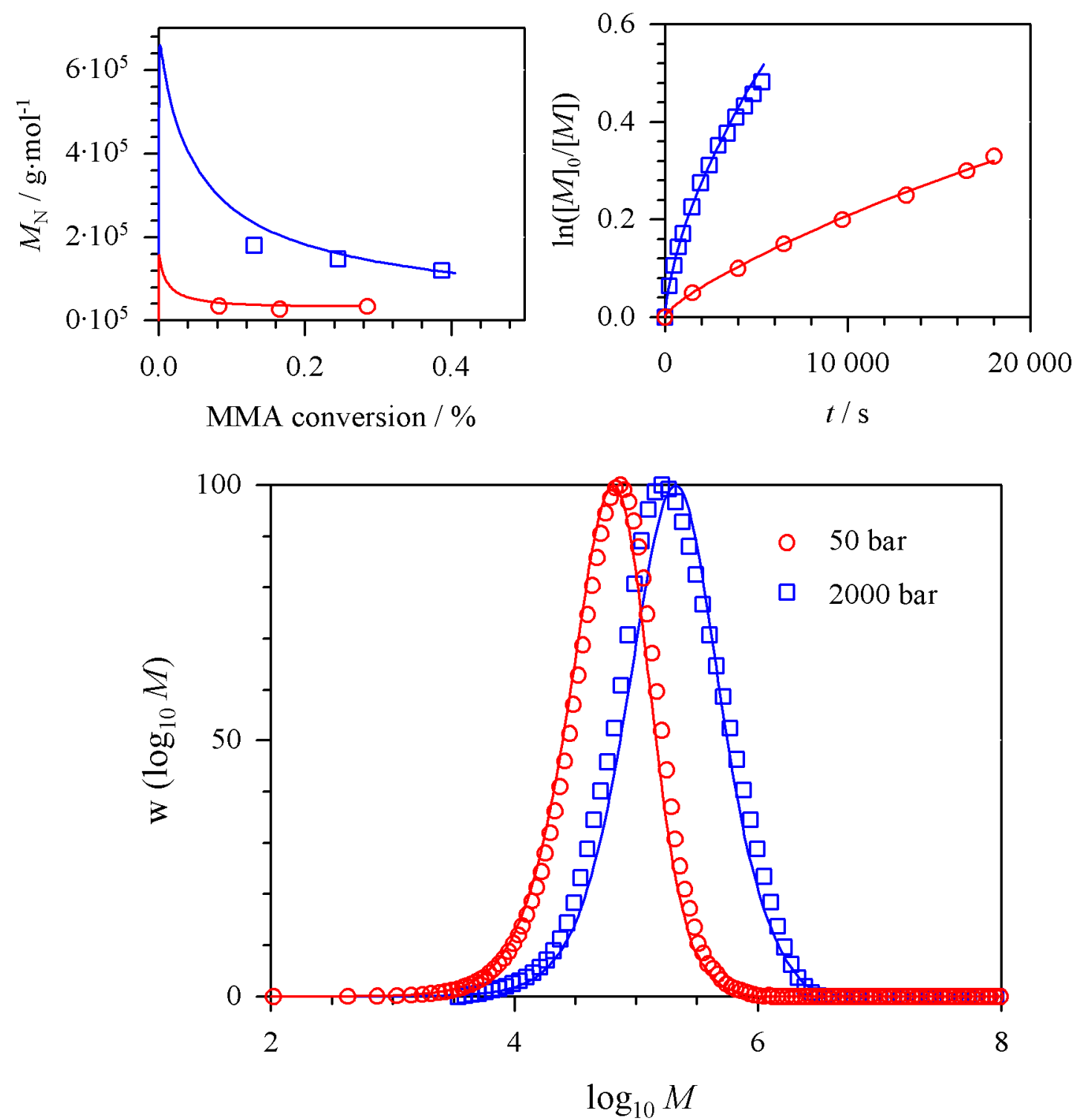

Fig. 6.29. Comparison of simulated (lines) with experimental data of DPAIO-mediated MMA polymerizations at $95^{\circ} \mathrm{C}$ and at pressures up to $2000 \mathrm{bar}$.

\subsection{Nitroxide-mediated ethene polymerization under high pressure}

Controlling ethene high-pressure polymerization regulated by persistent radicals is much more complicated than styrene or acrylates polymerizations. First of all, drastic temperature and pressure conditions are required for ethene homopolymerization and growing 
ethyl radicals are very reactive. An ethyl radical is no good leaving group which should unfavorably affect the equilibrium rate of the deactivation reaction. Finally ethene polymerization presents a complex kinetic situation at high temperature and pressure where transfer reactions, to monomer and to polymer, play an important role and $\beta$-scission processes also must be considered.

High-pressure ethene polymerization in the presence of persistent radicals was only reported in 1995 in a patent by Georges et al. ${ }^{70}$ but no experimental data were reported. The present work proposes a detailed experimental study of high-pressure ethene homopolymerization in the presence of various stable nitroxyl radicals. Different commercially available nitroxides of quite different reactivity, have been studied. Then new compounds have been developed in the course of the study by several groups.

Ethene polymerizations have been performed either with DTBP as high-temperature initiator associated to an excess of nitroxide or using an alkoxyamine over the temperature range $140-250^{\circ} \mathrm{C}$ at 2000 bar. The temperature of $140^{\circ} \mathrm{C}$ is the lowest temperature at which polyethylene is soluble in the monomer over the entire concentration range. Ethene concentration is spectroscopically monitored on-line as has been illustrated in Section 3.7.3.

\subsubsection{SG1, DTBN}

In this work, nitroxides have been classified in three different groups, depending on their thermal stability and reactivity toward radicals. DTBN and SG1 are the most reactive species but they are not stable at high temperatures. Ethene polymerizations carried out in the presence of DTBN did not show any characteristics of controlled polymerization. Even in the presence of a large excess of DTBN, the polymerization rate is not constant during polymerization, but termination reactions induce a strong slow down of the polymerization rate as is shown by the $\ln \left([M]_{0} /[M]\right)$ vs time evolution in Fig. 5.24. The inhibition period observed in the presence of a large excess of persistent radicals increases with the free nitroxide concentration, so that it was assumed that DTBN inhibits ethene polymerization as long as the persistent radical is in excess. If the nitroxide undergoes thermal degradation, the alkoxyamine thermal decomposition releases radicals which are not trapped any more and which can initiate polymerization. From this point the ethene polymerization is no longer controlled and molecular weights are determined by termination and transfer reactions. 
MWDs of polyethylene obtained by S-SG1 mediated ethene polymerization are also very broad. The molecular weights are not dependent on monomer conversion but are only dependent on the experimental conditions: particularly on $T, P$, and initiator concentration. At $190^{\circ} \mathrm{C}$ and 2000 bar, conversion independent molecular weights close to 110000 and

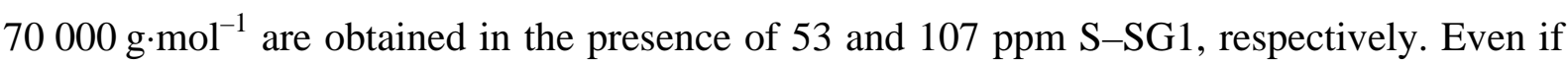
the conversion index $\ln \left([M]_{0} /[M]\right)$ slowly increases with time, ethene polymerization is not controlled in the presence of S-SG1. The low polymerization rates result in the low thermal degradation of the alkoxyamine which is associated with a low initiation rate. At high alkoxyamine concentrations (above $100 \mathrm{ppm}$ ) polymerization rates and molecular weights are independent of the S-SG1 concentration. The induced higher concentration of free radical is assumed to increase termination rate, resulting in polymerization rates and molar masses which are independent of S-SG1 concentration.

Very reactive nitroxides such as DTBN and SG1 are not stable enough to perform any high-temperature ethene polymerization. The bulkiness of this type of persistent radicals was expected to be sufficiently high to allow for a reversible dissociation of alkoxyamine. The inhibition period observed indicates that alkoxyamines are formed. Tordo et al. ${ }^{61}$ determined the decomposition temperature of SG1 by ESR to be around $140^{\circ} \mathrm{C}$ and the hexyl-SG1 dissociation temperature was determined to be close to $170^{\circ} \mathrm{C}$. At $190^{\circ} \mathrm{C}$, the competition of the thermal degradation of SG1 with the dissociation of hexyl-SG1 is responsible for the persistent radical effect to be absent and for a low rate of formation of growing radicals during the polymerization.

\subsubsection{TEMPO and TEMPO-derivatives}

TEMPO and TEMPO-derivatives are the most common mediators for nitroxidemediated polymerization. Ethene polymerization in the presence of TEMPO and TEMPOderivatives, such as hexyl-TEMPO and CXA, have been investigated over a wide range of pressures and temperatures. A typical ethene radical polymerization initiated by a peroxide ( $\mathrm{R}$ $=0$ ) is presented in Fig. 5.29. A very high polymerization rate is observed in the first minutes of the reaction. In the presence of TEMPO, the concentration of growing free radicals is lower. An excess of TEMPO leads to an inhibition period which depends on the concentration 
of TEMPO (see Fig. 5.29). These experiments prove that TEMPO is able to trap the growing radicals, produced by the DTBP decomposition and subsequent propagation of a few ethene molecules. The dissociation rate of the dormant species seems to be too low to allow for a controlled polymerization as was shown in Section 4.2. As soon as the excess of TEMPO is consumed, dormant species, which thermally and slowly decompose, will induce the low polymerization rate observed. Thus, molecular weights and polydispersities observed are not typical for a controlled polymerization. This slow release of radicals also explains the increase of the polymerization rate as compared to the one of spontaneous pure ethene polymerization.

TEMPO decomposition was supposed to be catalyzed by oxy-radicals. In order to avoid such catalysis, ethene polymerizations were carried out in the presence of hexylTEMPO. The conversion index increases with monomer conversion and allows to reach high conversions $\left(64 \%\right.$ at $230^{\circ} \mathrm{C}$ after 3 hours $)$. The $\ln \left([M]_{0} /[M]\right)$ vs time plots exhibit a low downward curvature. Broad MWDs are observed so that it is assumed that TEMPO decomposes which goes with a loss of control of the ethene polymerization.

\subsubsection{Thermal decomposition of nitroxides}

The thermal decomposition of the nitroxides under investigation was studied by gas chromatography and by ESR spectroscopy. From GC the nitroxide and alkoxyamine concentrations are accessible whereas ESR allows to follow the persistent radical concentration. Rate coefficients of the decomposition of DTBN, TEMPO and hexyl-TEMPO are plotted in Fig. 6.30.

As expected, DTBN is not very thermally stable. The activation energy of DTBN decomposition was calculated to be $142 \pm 4 \mathrm{~kJ} \cdot \mathrm{mol}^{-1}$. The activation energy of TEMPO decomposition was estimated to be $162 \pm 6 \mathrm{~kJ} \cdot \mathrm{mol}^{-1}$. Hexyl-TEMPO shows a good thermal stability. The $E_{\mathrm{a}}$ of hexyl-TEMPO decomposition is $182 \pm 7 \mathrm{~kJ} \cdot \mathrm{mol}^{-1}$ which is close to the dissociation energy of hexyl-TEMPO measured by Fischer to be between 180 and $190 \mathrm{~kJ} \cdot \mathrm{mol}^{-1}$. From ESR observations, it has been found that TEMPO decomposes to $40 \%$ within 5.2 hours at $200^{\circ} \mathrm{C}$ in diphenyl ether ${ }^{62}$. The rate coefficient of decomposition deduced from this measurement is also represented in Fig. 6.30 and agrees with the data from the GPC investigations. On the other hand, values of activation energy of TEMPO thermal decomposition are reported by Schmidt-Naake et al. ${ }^{63}$ to be $153 \mathrm{~kJ} \cdot \mathrm{mol}^{-1}$, and the rate 
coefficients are intermediate values between DTBN and TEMPO values determined in this work.

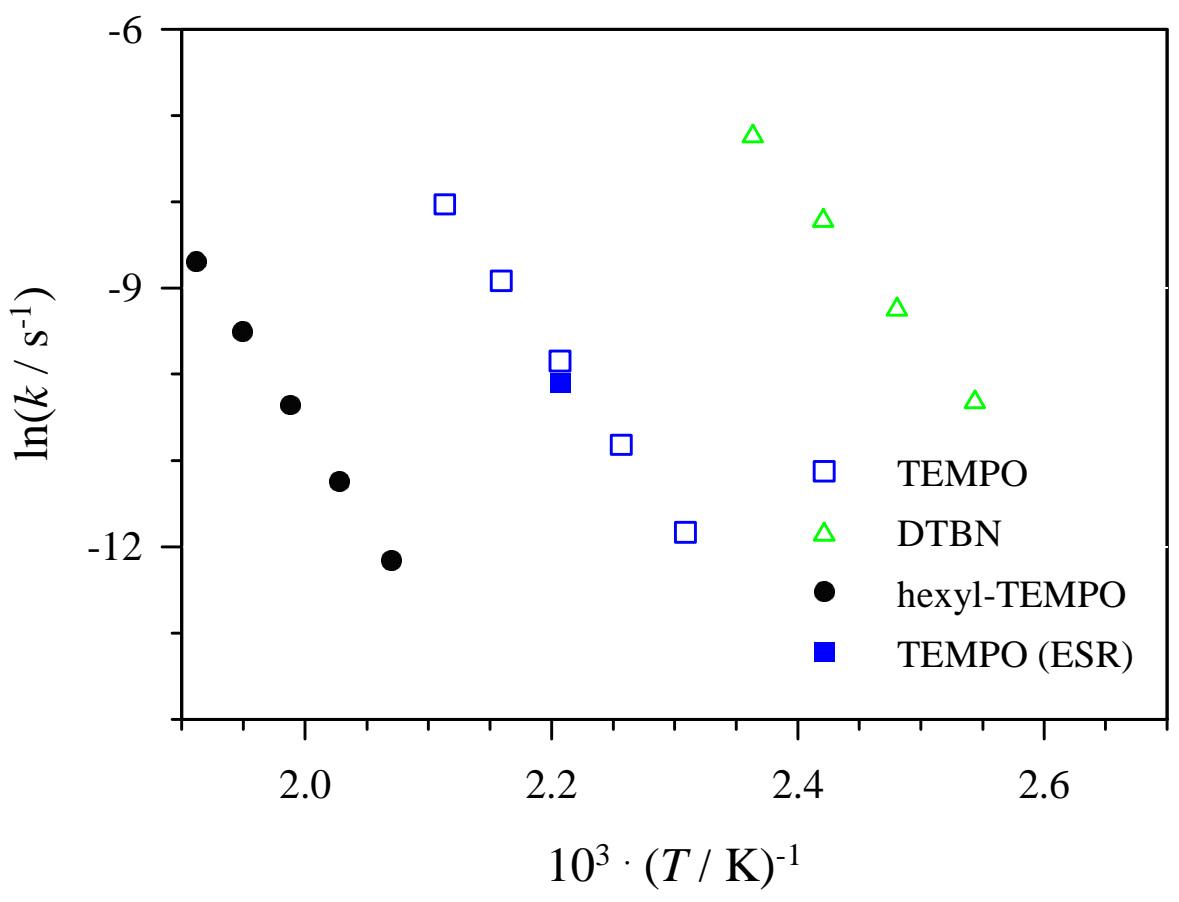

Fig. 6.30. Temperature dependence of the rate coefficients of nitroxides and alkoxyamines thermal decomposition. The data is from gas chromatography.

DTBN (and SG1) decomposes too rapidly at the temperatures applied for ethene polymerization as to allow for controlled ethene polymerization. TEMPO also decomposes at temperature required for the dissociation of the dormant species, so that no PRE is expected.

Further ESR experiments were performed to study the decomposition rate of DPAIO. The nitroxide decay was observed at $200^{\circ} \mathrm{C}$ and 1 bar in diphenyl ether. As was already reported by Greci et al. ${ }^{64}$, DPAIO is very stable. After 6 hours at $200^{\circ} \mathrm{C}$ less than $6 \%$ are consumed ${ }^{61}$.

\subsubsection{DPAIO}

Experimental investigations show that a fast ethene polymerization rate is observed at fairly low temperatures at 2000 bar in the presence of alkoxyamine hexyl-DPAIO. In fact, at $160^{\circ} \mathrm{C}$ where DPAIO is thermally stable, the ethene polymerization reaches about $25 \%$ 
conversion in 30 minutes. At high alkoxyamine concentration, the polymerization rate was shown to be independent of the alkoxyamine concentration. As it is described in Section 5.5.4, ethene polymerization in the presence of hexyl-TEMPO is characterized by a linear evolution of $\ln \left([M]_{0} /[M]\right)$ with time, which is explained by the low hexyl-TEMPO rate of decomposition during the course of the polymerization. In case of hexyl-DPAIO-mediated polymerization, also a linear $\ln \left([M]_{0} /[M]\right)$ versus time correlation is observed (see Fig. 5.36). This conversion may be due to the reversible dissociation of the dormant species but may also be induced by the alkoxyamine thermal decomposition. Because of equimolar quantities of growing and persistent radicals, polymerization starts without any inhibition period.

ESR investigations showed a very good thermal stability of DPAIO at $200^{\circ} \mathrm{C}$ for many hours. Spontaneous ethene polymerization is negligible under these conditions. ESR investigations into hexyl-DPAIO were carried out for many hours at $200^{\circ} \mathrm{C}$ in tetradecane and no signal of the persistent radical has been detected.

Ethene polymerizations were also performed at different hexyl-DPAIO concentrations at $160^{\circ} \mathrm{C}$ and 2000 bar (see Fig. 5.37). Almost the same polymerization rate was observed at alkoxyamine concentrations between 50 and $400 \mathrm{ppm}$. In cases where thermal decomposition of the alkoxyamine induces most of the polymerization, it is assumed that the polymerization rate should depend on radical concentration and thus on nitroxide concentration. Only at very low alkoxyamine concentrations the polymerization rate is lower.

Broad MWDs were observed and polydispersities in the range of 3 to 6 are obtained in hexyl-DPAIO mediated ethene polymerization. A slight increase of the molecular weights with monomer conversion has also been observed. The evolution of number molecular weight plotted in Fig. 6.31 undergoes a very slight increase with monomer conversion, but does not pass through the origin. Experimental $M_{\mathrm{N}}$ seems to be strongly dependent on the hexyl-DPAIO concentration and differs very much from molecular weights expected for controlled polymerization (see Table 6.32). Thus molecular weight evolution does not suggest that a controlled polymerization takes place. Considering $M_{\mathrm{N}}$ evolution, hexyl-DPAIO seems to play the role of a conventional chain-transfer agent. 


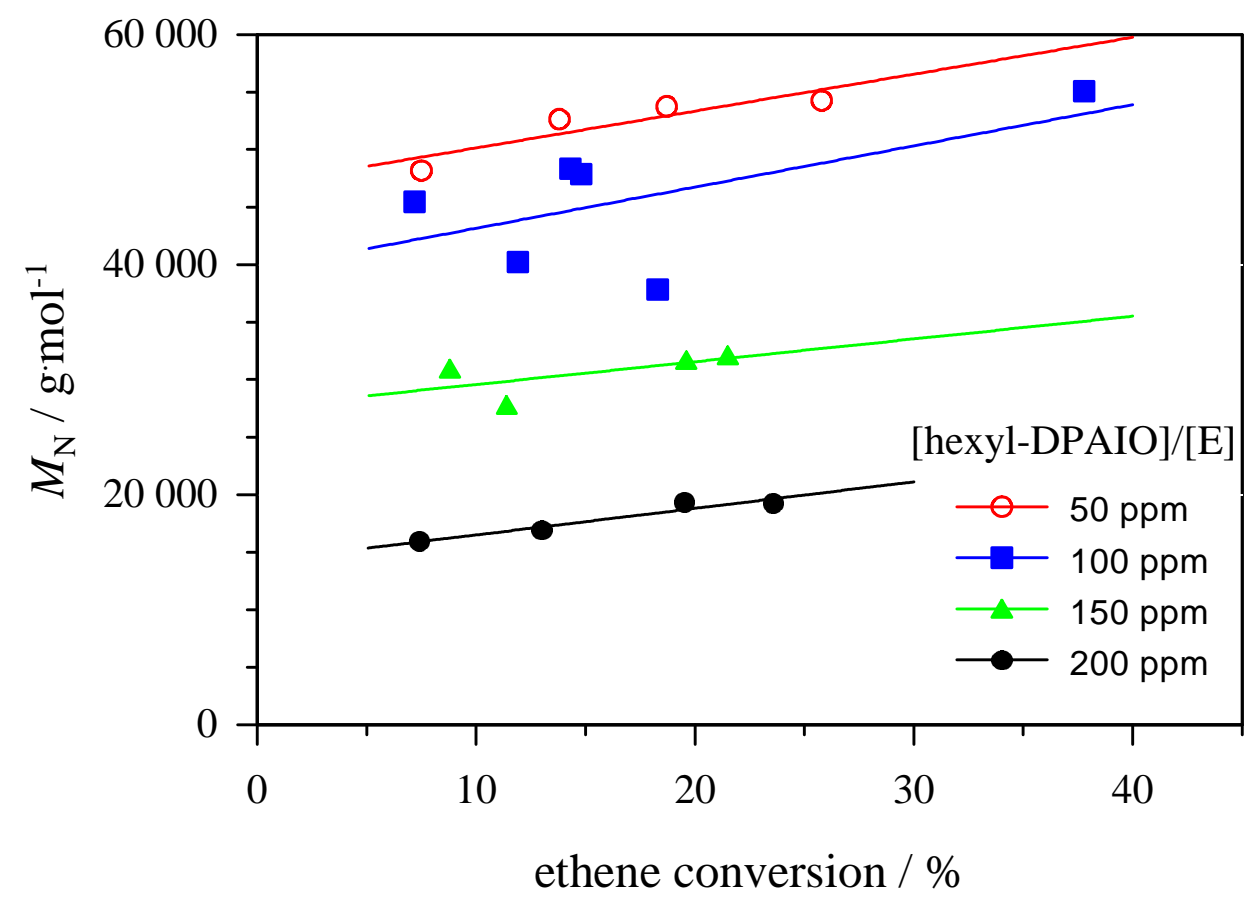

Fig. 6.31. Number average molecular weight as a function of monomer conversion for ethene polymerizations at $160^{\circ} \mathrm{C}$ and 2000 bar in the presence of different concentrations of hexyl-DPAIO.

\begin{tabular}{|c|c|c|c|c|}
\hline \multirow[t]{2}{*}{$\begin{array}{c}\text { [hexyl-DPAIO] } \\
/[\text { ethene }]\end{array}$} & \multicolumn{2}{|c|}{$\begin{array}{c}\text { experimental data } \\
\mathrm{g} \cdot \mathrm{mol}^{-1}\end{array}$} & \multicolumn{2}{|c|}{$\begin{array}{c}\text { estimated data } \\
\mathrm{g} \cdot \mathrm{mol}^{-1}\end{array}$} \\
\hline & $M_{\mathrm{N}}$ & $M_{\mathrm{W}}$ & $M_{\mathrm{N} \text { theory }}$ & $\begin{array}{l}M_{\mathrm{W} \text { theory }} \\
(I p=1.2)\end{array}$ \\
\hline $50 \mathrm{ppm}$ & 52000 & 290000 & 112000 & 134400 \\
\hline 100 ppm & 46000 & 230000 & 56000 & 67200 \\
\hline 200 ppm & 31000 & 150000 & 28000 & 33600 \\
\hline $400 \mathrm{ppm}$ & 26000 & 123000 & 14000 & 16800 \\
\hline
\end{tabular}

Table 6.32. Experimental and estimated molecular weights at $20 \%$ conversion for ethene polymerizations at $160^{\circ} \mathrm{C}, 2000$ bar and different concentrations of hexyl-DPAIO.

Assuming $M_{W}$ to be less sensitive toward oligomers , an increase of $M_{W}$ with monomer conversion is observed but experimental data do not agree with the theoretically expected ones. MWDs plotted in Fig. 5.39 present a clear bimodal distribution for ethene polymerization at $50 \mathrm{ppm}$ hexyl-DPAIO. This phenomenon was so clearly observed only at 
$50 \mathrm{ppm}$ : the signal at high molecular weight is only slightly visible at low alkoxyamine concentration and not any more above $100 \mathrm{ppm}$. At low concentration (up to $50 \mathrm{ppm}$ ) a monomodal MWD was also observed at high molar mass. Experimental MWDs are plotted in Fig. 6.33.

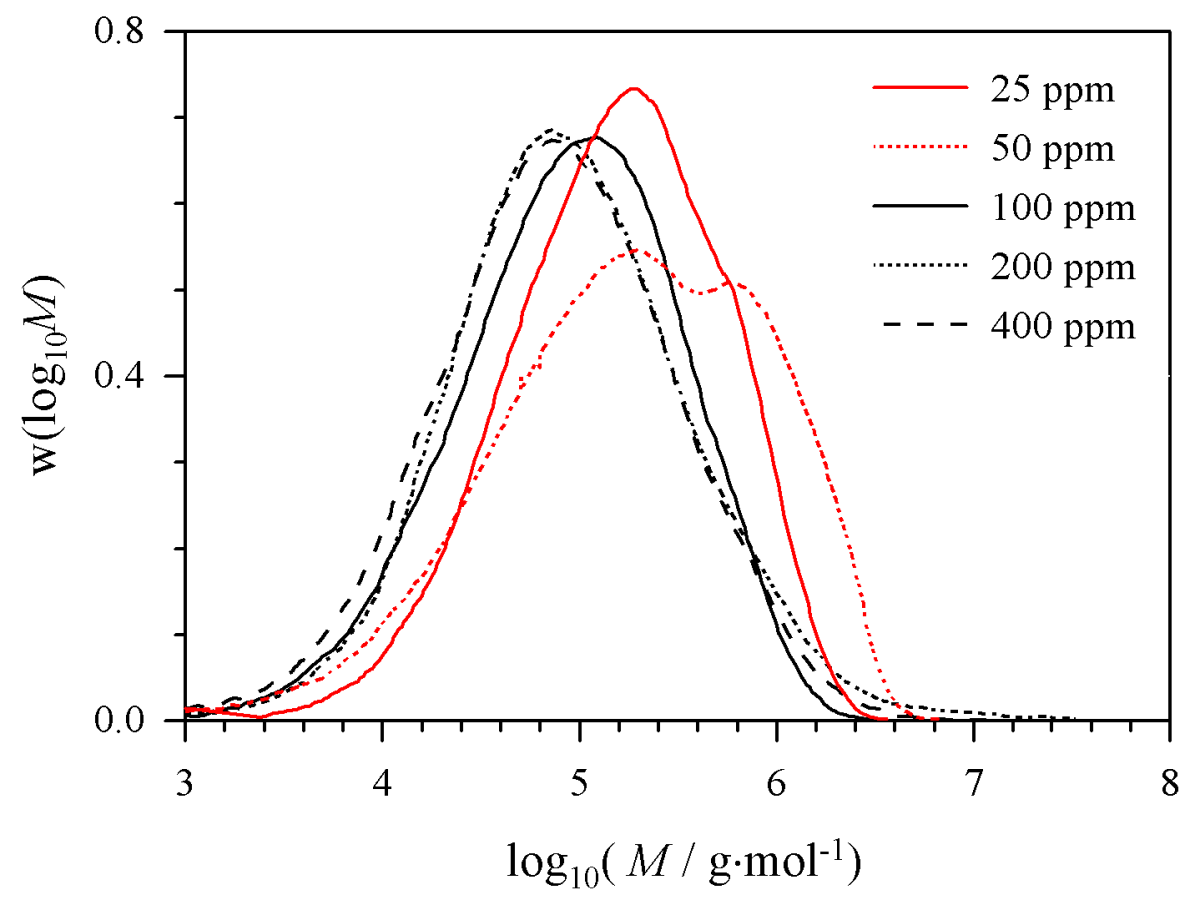

Fig. 6.33. Experimental molecular weight distributions at $20 \%$ conversion of polyethylene from ethene polymerizations at $160^{\circ} \mathrm{C}, 2000$ bar and different concentrations of hexyl-DPAIO.

In order to characterize the different MWDs observed, PREDICI ${ }^{\circledR}$ simulations of the ethene polymerization mediated by hexyl-DPAIO at $160^{\circ} \mathrm{C}$ and 2000 bar were performed and MWDs obtained are compared with the experimental ones. The model for nitroxide-mediated ethene polymerization used has already been presented in Section 4.2. Spontaneous ethene polymerization under these conditions is negligible. The next simulation does not consider any DPAIO thermal decomposition. The combination rate coefficient $k_{\mathrm{c}}$ was set to be close to the termination rate coefficient $k_{\mathrm{t}}$. The dissociation rate coefficient $k_{\mathrm{d}}$ of hexyl-DPAIO was determined by fitting the experimental polymerization rate. 
conditions

$160^{\circ} \mathrm{C}, 2000$ bar, $100 \mathrm{ppm}$ hexyl-DPAIO $k_{\mathrm{c}} / \mathrm{L} \cdot \mathrm{mol}^{-1} \cdot \mathrm{s}^{-1}$

$10^{8}$ $k_{\mathrm{d}} / \mathrm{s}^{-1}$

$7 \cdot 10^{-2}$

The simulation yields narrow MWDs (Ip around 1.40). $M_{N}$ and $M_{W}$ increase linearly with monomer conversion and agree with theoretical predictions. The concentration of dormant species is also much higher than the dead polymer concentration. Controlled polymerization thus seems to be possible under these conditions. This simulation however does not allow for any fit of the measured molecular weight data.

A second simulation was performed which allows for some thermal decomposition of the alkoxyamine hexyl-DPAIO to produce growing radicals. The rate coefficient of hexylDPAIO thermal decomposition has been chosen such that more than $99 \%$ of the alkoxyamine are decomposed in one hour. The polydispersity increases to about two. High molecular weights components are obtained which do not increase in molecular weight with monomer conversion. The simulations show that the thermal decomposition induces broad MWDs and high molecular weights and controlled polymerization allows to obtain narrow MWDs and low molecular weights. This may explain the experimental bimodal MWD observed previously. Part of the polymer, in particular at low molecular weight, may be from controlled polymerization and another is from the thermal decomposition of the alkoxyamine, leading to high molecular weight polymer. At low hexyl-DPAIO concentrations, dormant species at low concentration are hidden by polymer of high molecular weight, induced by alkoxyamine decomposition, resulting in a monomodal distribution. At high alkoxyamine concentration, the associated high concentration of free radicals is assumed to increase transfer and termination reactions so that lower molecular weight material is observed.

Further experiments were carried out in the presence of $100 \mathrm{ppm}$ of hexyl-DPAIO and an excess of $10 \mathrm{~mol} \%$ of nitroxide DPAIO and no characteristics of controlled polymerization has been observed.

It should be noted that, even DPAIO is thermally stable, whereas the alkoxyamine hexyl-DPAIO and the adduct PE-DPAIO undergo a quite fast thermal degradation. Polyethylene samples produced in the presence of hexyl-DPAIO under different conditions were investigated by ESR. No signal of persistent radical was observed at $160^{\circ} \mathrm{C}$, so it is assumed that the polyethylene samples are no living polymers and are not DPAIO-capped 
macroradicals. Further ESR investigations for the determination of cleavage temperature of alkoxyamines have been performed ${ }^{1}$ and are presented in the next section.

\subsubsection{Determination of the cleavage temperature of alkoxyamines}

The cleavage temperatures of the alkoxyamines have been estimated by ESR in deoxygenated tetradecane at concentration of $10^{-1} \mathrm{M}^{61}$. The cleavage temperature corresponds to the temperature at which the ESR signal appears and increases with time. Hexyl-DPAIO was investigated up to $200^{\circ} \mathrm{C}$ and no ESR signal has been observed. It is assumed that the difference of cleavage temperature between hexyl-SG1 and hexyl-DPAIO is of the same order of magnitude as the difference observed between styryl-SG1 and styrylDPAIO. Based upon this assumption a cleavage temperature of about $230^{\circ} \mathrm{C}$ is expected for hexyl-DPAIO.

\begin{tabular}{cc}
\hline styryl-SG1 & styryl-DPAIO \\
$T_{\mathrm{c}}=60^{\circ} \mathrm{C}$ & $T_{\mathrm{c}}=120^{\circ} \mathrm{C}$ \\
\hline hexyl-SG1 & hexyl-DPAIO \\
$T_{\mathrm{c}}>170^{\circ} \mathrm{C}$ & $T_{\mathrm{c}}>230^{\circ} \mathrm{C}$ (estimated) \\
\hline
\end{tabular}

Table 6.34. Cleavage temperature of different alkoxyamines determined by ESR in tetradecane and estimation of the cleavage temperature of hexyl-DPAIO.

Persistent radicals, such as nitroxyl radicals, are easily detected by ESR whereas other radicals from thermal decomposition of nitroxide are difficult to be detected. Thus it may be possible that hexyl-DPAIO decomposes by pathways other than by breaking the $\mathrm{C}-\mathrm{O}$ bond could yield no or no strong ESR signal. Thus experiments were carried out, following the hexyl-DPAIO concentration by Thin Layer Chromatography (TLC) ${ }^{62}$. A $10^{-3} \mathrm{M}$ hexylDPAIO concentrated solution in dodecane has been heated to $160^{\circ} \mathrm{C}$ and the alkoxyamine concentration, and eventually products, were measured by TLC. About $50 \%$ of the alkoxyamine disappeared before the temperature of $160^{\circ} \mathrm{C}$. After one hour at $160^{\circ} \mathrm{C}$, there is no hexyl-DPAIO left. The major product issued from the thermal degradation the corresponding amine presented in Figure 6.35, so that the hexyl-DPAIO decomposes via $\mathrm{N}-\mathrm{O}$ bond cleavage. As a conclusion from this series of ESR investigations, it is assumed that 
the alkoxyamine hexyl-DPAIO undergoes a thermal degradation at a temperature much lower than the temperature required to open the $\mathrm{O}-\mathrm{C}$ bond. Obviously the bond dissociation energy $\mathrm{BDE}$ of the $\mathrm{N}-\mathrm{O}$ bond is lower than the $\mathrm{BDE}$ of the $\mathrm{O}-\mathrm{C}$ bond.

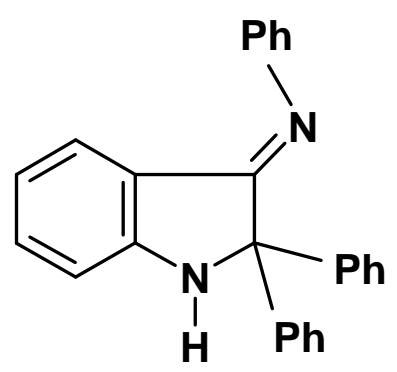

Fig. 6.35. Amine issued from hexyl-DPAIO thermal decomposition.

\subsubsection{Study of BDE by molecular modeling of a series of indolinic aminoxyl radicals}

The control of radical polymerizations depends on the reversible dissociation of a growing chain capped by a nitroxide moiety. When either $k_{\mathrm{d}}$ increases or $k_{\mathrm{c}}$ decreases, the polymerization time decreases. $K$ is a thermodynamic parameter and is related to the bond dissociation enthalpy, BDE, of the $\mathrm{C}-\mathrm{O}$ bond (see Scheme 6.36).

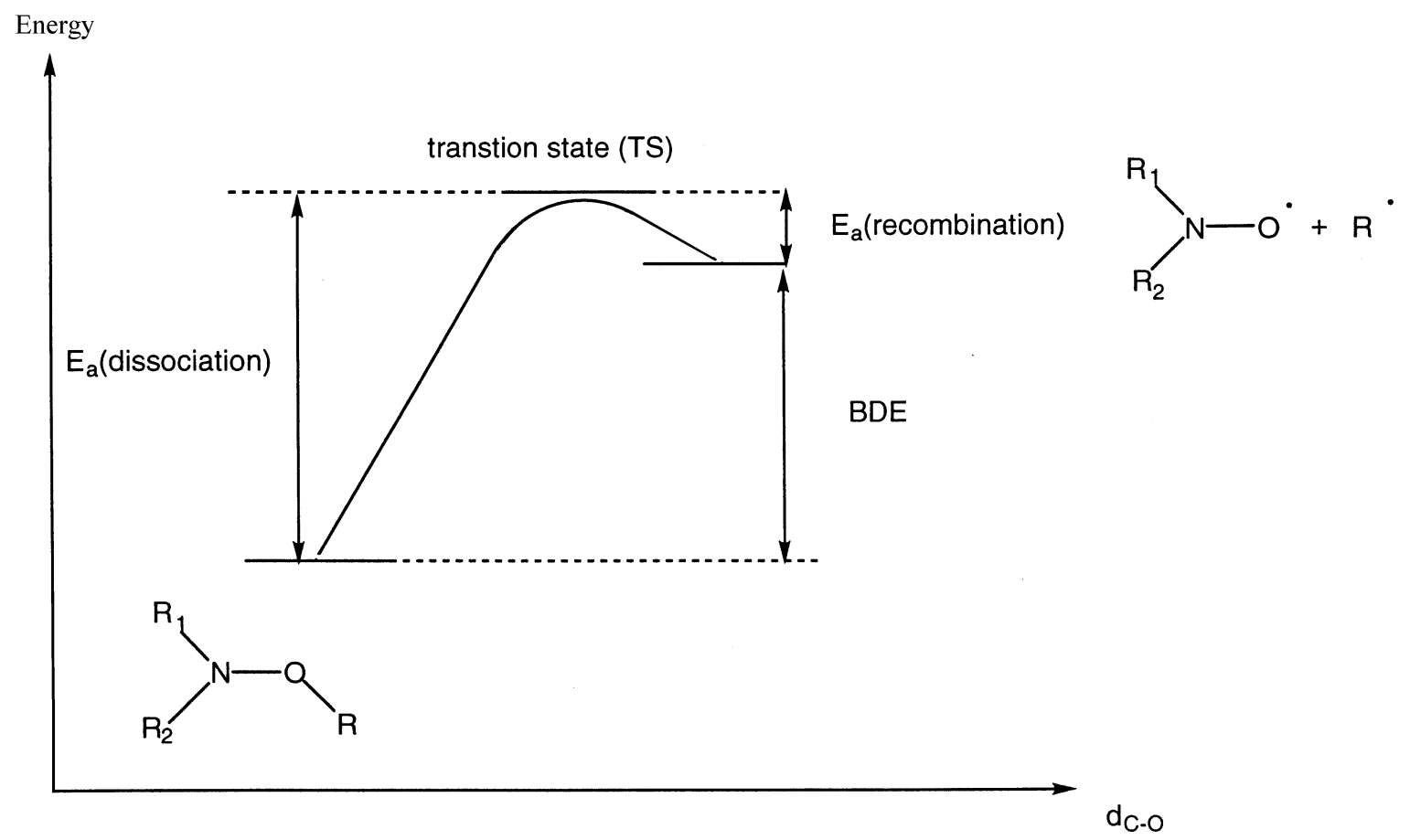

Scheme. 6.36. $\quad$ Pathway of the homolytic dissociation of an alkoxyamine. 
The reversible homolysis of an alkoxyamine is influenced by steric and/or electronic factors of the nitroxyl and polymer moieties. The steric congestion is reduced by the dissociation, so that $k_{\mathrm{d}}$ and $K$ increase with the steric hindrance around the $\mathrm{C}-\mathrm{O}$ bond. If the nitroxide or the polymer radical is stabilized by delocalization of the unpaired electron, the BDE decreases.

It has been established that the BDE of a nitroxide capped polymer alkoxyamine is close to the BDE in a model alkoxyamine. Thus molecular modeling of the BDE of alkoxyamines can provide thermodynamic information to estimate the importance of different factors on the dissociation equilibrium. These estimations may be very helpful to design new nitroxides for control of free-radical polymerization of a given monomer.

Quantitative information about radical combination reactions may be provided by abinitio molecular orbital calculations ${ }^{65}$. The ab-initio calculations allow to obtain information on reaction energy barriers (see Fig. 6.36). The figure shows a schematic potential energy profile corresponding to the dissociation of an alkoxyamine, proceeding via a transition structure (TS) to produce a radical $\mathrm{R}^{\bullet}$ and a nitroxide. The calculations allow to obtain complete geometries, bond and torsional angles, of all species. It is also possible to determine thermochemical quantities such as reaction barrier and exothermicity. Calculations may be carried out using either a restricted (RHF) or unrestricted (UHF) Hartree-Fock methods ${ }^{66}$.

Considering the size of the studied molecules, Tordo et al ${ }^{67}$ used semi-empirical methods (AMPAC software) for these calculations. It was already shown that PM3 parametrization offers the best description of alkoxyamines and nitroxides (compared to AM1 and SAM1 parametrizations). At first, the BDE calculations were performed at the RHF level in order to avoid spin contamination in the conjugated nitroxides. The UHF BDEs are known to be underestimated but this method offers realistic relative BDEs for this kind of compounds. The polyethylene chain was simulated with an $n$-hexyl chain and the preferred conformer of each alkoxyamine was found after a simulated annealing. Because of the large number of variables, some negligible internal coordinates (like $\mathrm{C}-\mathrm{H}$ stretchings or the deformations of the phenyl rings) were frozen during the simulated annealing.

Previous calculated BDE of alkoxyamines with conjugated leaving radicals have shown a good correlation with the experimental temperatures reported in Fig. 6.37. The cleavage temperatures are determined by ESR. 


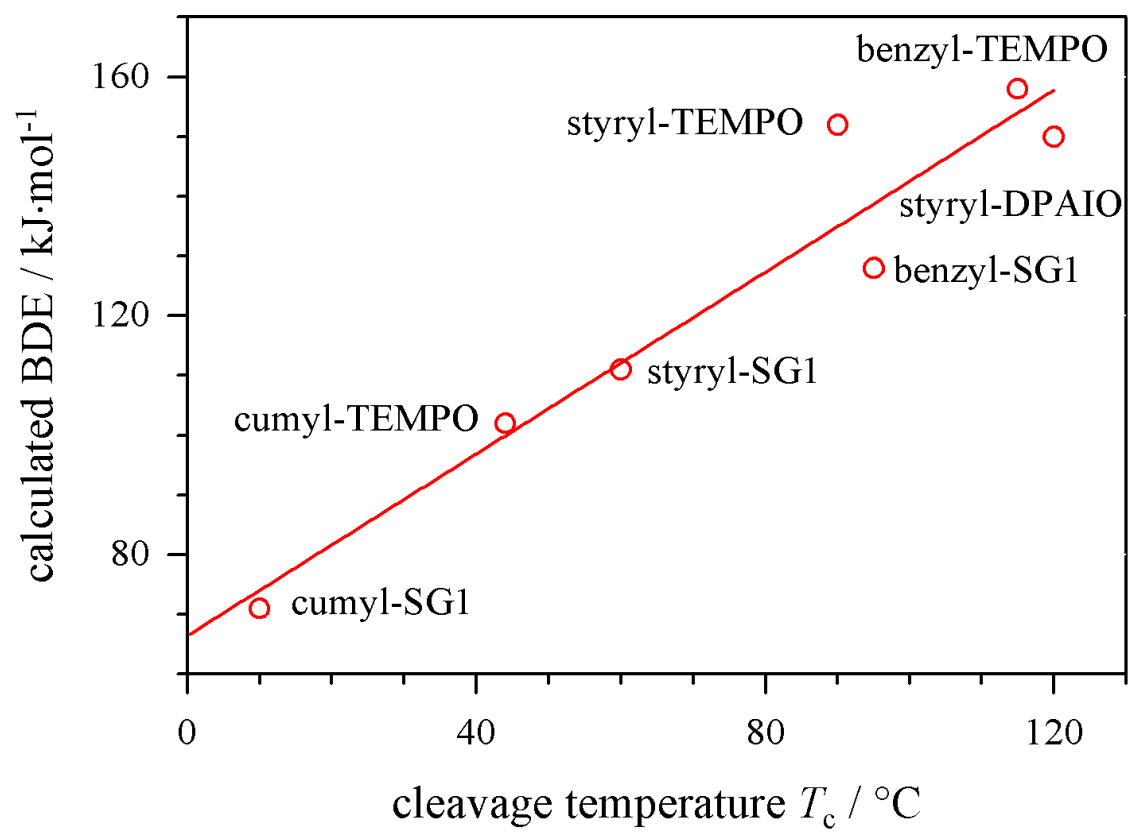

Fig. 6.37. Correlation between the calculated BDE and the cleavage temperature of the $\mathrm{C}-\mathrm{O}$ bond in alkoxyamines with conjugated leaving groups.

The BDE of the $\mathrm{O}-\mathrm{C}$ bond in hexyl-DPAIO was calculated to be around $185 \pm 10 \mathrm{~kJ} \cdot \mathrm{mol}^{-1}$. The validity of the BDE calculations have been verified on a small series of nitroxide-alkyl alkoxyamines. This correlation which is reported in Fig. 6.38 allows the estimation of the cleavage temperature of the $\mathrm{C}-\mathrm{O}$ bond in hexyl-DPAIO at around $210^{\circ} \mathrm{C}$.

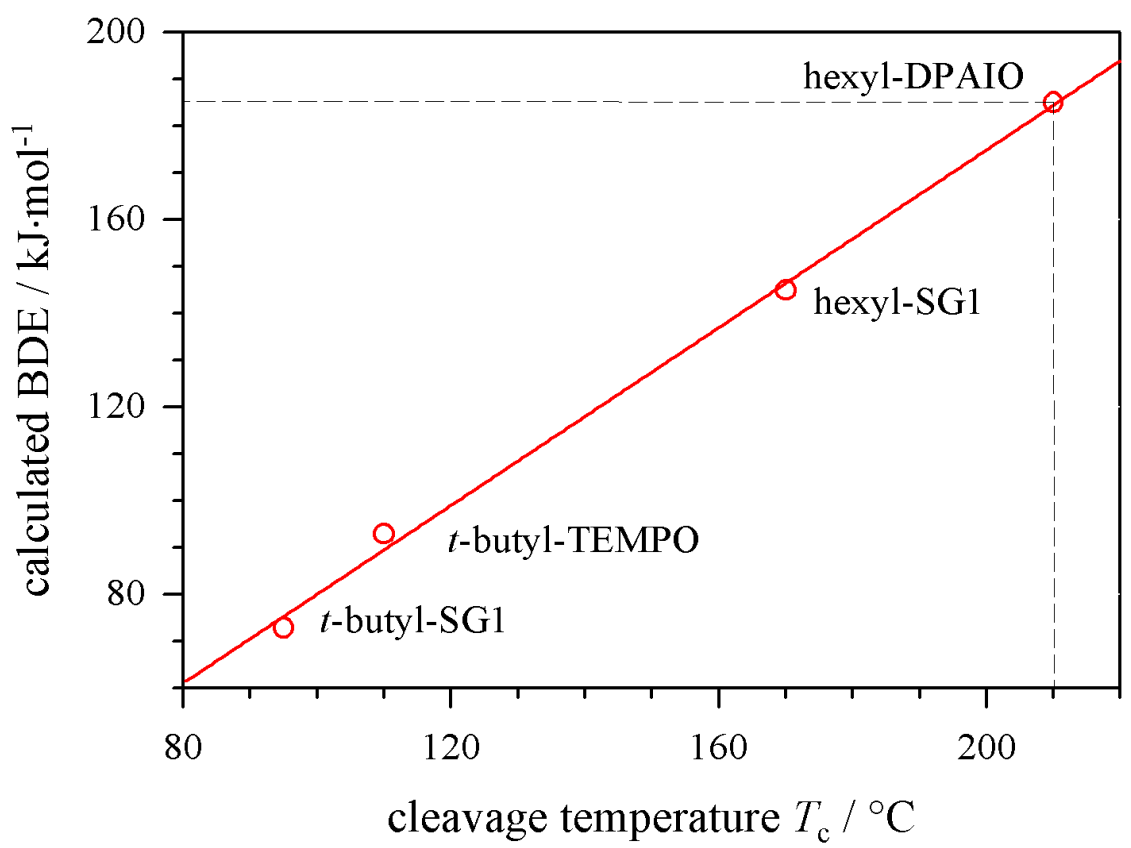

Fig. 6.38. Estimation of the cleavage temperature of the $C-O$ bond in hexyl-DPAIO. 
The experimental study of thermal degradation shows that it is the $\mathrm{N}-\mathrm{O}$ bond of the alkoxyamine which breaks instead of the $\mathrm{C}-\mathrm{O}$ bond which should break in order to allow for control of the radical polymerization. Further BDE calculations were performed in order to compare the strength of the $\mathrm{N}-\mathrm{O}$ and $\mathrm{C}-\mathrm{O}$ bonds. BDE calculations for the two bonds of different alkoxyamines are reported in Table 6.39. Firstly, as experimentally observed, the $\mathrm{N}-\mathrm{O}$ bond was found to be weaker than the $\mathrm{C}-\mathrm{O}$ bond in the case of hexyl-DPAIO. The same conclusion is reached for alkoxyamines hexyl-4a and hexyl-4b (see structures in Scheme 6.40) . The weakness of the $\mathrm{N}-\mathrm{O}$ bond is strongly dependent on steric hindrance as can be seen from a comparison of $4 \mathrm{a}$ and $4 \mathrm{~b}$. The presence of bulky substituents on the nitroxide induces a strong decrease of the $\mathrm{N}-\mathrm{O}$ BDE. The $\mathrm{C}-\mathrm{O}$ bond in hexyl-nitroxide alkoxyamines appears to be extremely strong as compared with that in styryl-TEMPO and other TEMPO-derived alkoxyamines (Table 6.39). These calculations also show that the $\mathrm{C}-\mathrm{O}$ bond is weaker than the $\mathrm{N}-\mathrm{O}$ bond in S-TEMPO, allowing a satisfactory controlled polymerization.

\begin{tabular}{lcccccc}
\hline & $\begin{array}{c}\text { hexyl- } \\
\text { DPAIO }\end{array}$ & hexyl-4a & hexyl-4b & $\begin{array}{c}\text { benzyl- } \\
\text { TEMPO }\end{array}$ & $\begin{array}{c}\text { styryl- } \\
\text { TEMPO }\end{array}$ & $\begin{array}{c}\text { cumyl- } \\
\text { TEMPO }\end{array}$ \\
\hline $\begin{array}{l}\text { BDE C-O } \\
\mathrm{kcal} \cdot \mathrm{mol}^{-1}\end{array}$ & 31.2 & 29.5 & 31.7 & 20.0 & 15.7 & 10.2 \\
\hline $\begin{array}{l}\mathrm{BDE} \mathrm{N}-\mathrm{O} \\
\mathrm{kcal} \cdot \mathrm{mol}^{-1}\end{array}$ & 16.4 & 6.4 & 1.4 & 16.5 & 17.3 & 20.1 \\
\hline
\end{tabular}

Table 6.39. Estimation of $B D E$ of the $\mathrm{C}-\mathrm{O}$ and $\mathrm{N}-\mathrm{O}$ bonds in different alkoxyamines (see text for the method used)

BDE calculations have been performed on a series of indolinic aminoxyl radicals with various substituents. For the nitroxides $2 \mathrm{a}-\mathrm{c}$, the $\mathrm{BDE}$ of the $\mathrm{C}-\mathrm{O}$ bond should be reduced by their greater stabilization due to the extended delocalization of the unpaired electron. Moreover, a favorable steric factor is introduced in $2 \mathrm{c}$. In the $3 \mathrm{a}-\mathrm{c}$ series, the steric hindrance around the aminoxyl moiety is varied and the BDE should decrease due to destabilization of the alkoxyamine. In the case of nitroxide 4 , the aminoxyl function is a six-membered ring and the BDE of such alkoxyamine is known to be lower than those involving five-membered rings. 
The calculations using UHF method show a decrease of the BDE of the $\mathrm{C}-\mathrm{O}$ bond in hexyl-2a, hexyl-2b, hexyl-2c relative to hexyl-DPAIO. As expected, the increase of the steric hindrance in $3 \mathrm{~b}$ and $3 \mathrm{c}$ results in a slightly decrease of the BDE relative to the BDE of hexyl-DPAIO. The BDE of hexyl-4 is also lowered at the RHF and UHF levels but not as much as it was expected considering the stabilization of the nitroxide.

The calculations however show that the $\mathrm{N}-\mathrm{O}$ bond in the different alkoxyamines hexyl-2a-c, hexyl-3a-c, and hexyl-4a-b was also shown to be much weaker than the $\mathrm{C}-\mathrm{O}$ bond (Tordo et al. ${ }^{67}$ ). No substituents were found to be able to strongly increase the strength of the $\mathrm{N}-\mathrm{O}$ bond.<smiles>[O-]N1c2ccccc2/C(=N\c2ccccc2)C1(c1ccccc1)c1ccccc1</smiles>

DPAIO<smiles>[O-]N1c2c(ccc3ccccc23)/C(=N\c2ccccc2)C1(c1ccccc1)c1ccccc1</smiles>

2c<smiles>Cc1cc(C)c(C2(c3ccccc3)/C(=N\c3ccccc3)c3cccc(C(C)(C)C)c3N2O)c(C)c1</smiles><smiles>[O-]N1c2cc3ccccc3cc2/C(=N\c2ccccc2)C1(c1ccccc1)c1ccccc1</smiles>

$2 a$<smiles>Cc1cccc2c1N([O-])C(c1ccccc1)(c1ccccc1)/C2=N\c1ccccc1</smiles>

$3 a$<smiles>[O-]N1c2ccccc2-c2ccccc2C1(c1ccccc1)c1ccccc1</smiles>

$4 a$<smiles>[O-]N1c2ccc3ccccc3c2/C(=N\c2ccccc2)C1(c1ccccc1)c1ccccc1</smiles>

2b

$\mathrm{Ph}$<smiles>CCCc1cccc2c1N([O])C(c1ccccc1)(c1ccccc1)C2=NC</smiles>

$3 b$<smiles>CC(C)(C)c1cccc2c1N([O])C(c1ccccc1)(c1ccccc1)c1ccccc1-2</smiles>

4b

Scheme 6.40. $\quad$ Series of studied indolinic aminoxyl radicals. 


\subsection{RAFT ethene polymerization under high pressure}

Recently, an effective and versatile living radical polymerization process was reported by Rizzardo et al. which functions by reversible addition-fragmentation chain transfer. The process is performed simply by adding a suitable thiocarbonylthio compound to a conventional free-radical polymerization. Rizzardo et al. ${ }^{68}$ reported in detail on the polymerization of methacrylates, styrenes, acrylates, acrylamides and vinyl actetate and offered a selection of preferred thiocarbonylthio compounds for particular monomer types.
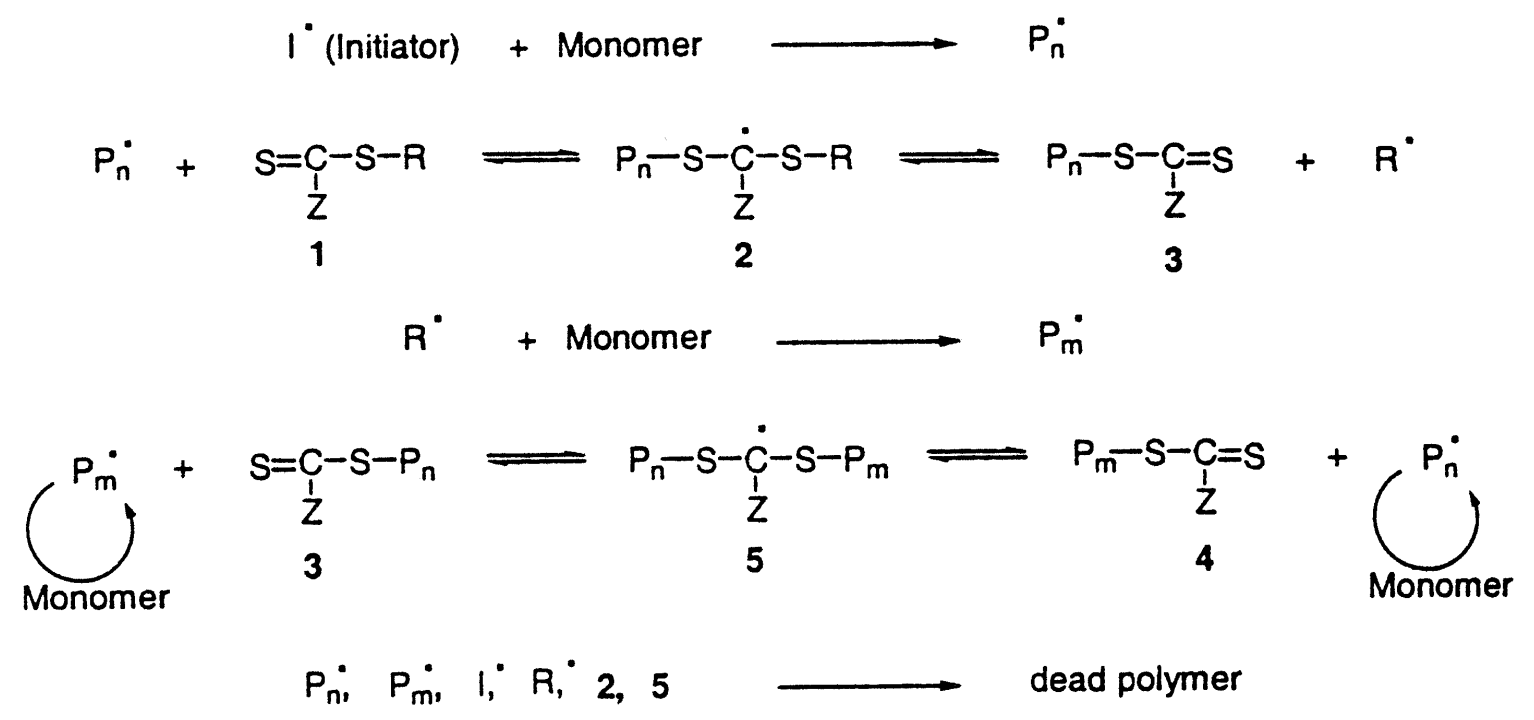

Scheme 6.41. Mechanism proposed for RAFT polymerization.

The mechanism of the RAFT process is believed to involve a series of reversible addition-fragmentation steps as shown in Scheme 6.41. Addition of a growing radical $\mathrm{P}_{\mathrm{n}}$, issued from the initiation step, to the thiocarbonylthio compound $\mathbf{1}$ gives the adduct radical $\mathbf{2}$ which fragments to polymeric thiocarbonylthio compound $\mathbf{3}$ and to a new radical $\mathrm{R}$. The radical $\mathrm{R}$ adds a monomer molecule to give a new propagating radical $\mathrm{P}_{\mathrm{m}}$. Subsequent additional-fragmentation steps establish an equilibrium between the propagating radicals $\mathrm{P}_{\mathrm{n}}$ and $\mathrm{P}_{\mathrm{m}}$ and the dormant polymeric thiocarbonylthio compound $\mathbf{3}$ and $\mathbf{4}$ by way of the intermediate radical 5. Equilibration of the growing chains gives rise to a narrow molecular weight distribution.

For RAFT polymerization to function effectively, the choice of the thiocarbonylthio compound $\mathbf{1}$ is extremely important. The requirements are high rate constants for both the addition of propagating radicals to the thiocarbonylthio compound $\mathbf{1}, \mathbf{3}$ and $\mathbf{4}$ and 
fragmentation of the intermediate radicals $\mathbf{2}$ and $\mathbf{5}$, relative to the rate constant for propagation. In other words, the species 1, 3 and 4 must have high chain transfer constants ${ }^{68}$.

In general terms, the rates of addition of radicals to thiocarbonylthio compound $\mathbf{1}, \mathbf{3}$ and $\mathbf{4}$ are strongly influenced by the substituent Z. It appears that radical stabilizing groups such as phenyl enhance the rate of addition of radicals to the $\mathrm{C}=\mathrm{S}$ bond. On the other hand, when $\mathrm{Z}$ is a nitrogen or oxygen substituent, the reagents are practically inert in the polymerization of acrylate, methacrylate and styrene monomers. This is attributed to a lowering of the double bond character of the $\mathrm{C}=\mathrm{S}$ bond by conjugation of the lone pair of electrons on the heteroatom substituent.

A high rate of fragmentation of radical $\mathbf{2}$ to the polymeric thiocarbonylthio species $\mathbf{3}$ is achieved by selecting a substituent $\mathrm{R}$ that is a good homolytic leaving group relative to the polymer chain $\mathrm{P}_{\mathrm{n}}$. The homolytic leaving ability of a group $\mathrm{R}$ appears to increase with increasing radical stability of the expelled radical $\mathrm{R}$ and increasing steric bulkiness of $\mathrm{R}$. It should also be noted that the radical $\mathrm{R}$ expelled from $\mathbf{1}$ must be sufficiently reactive to initiate polymerization efficiently ${ }^{68}$.

The data in Fig. 5.43, show that ethene polymerizations in the presence of the RAFT agent do not show any characteristics of a controlled polymerization. Broad polydispersities (from 2.2 up to 3) and $M_{\mathrm{N}}$ being independent of conversion are observed, so that the RAFT agent seems to play the role of a simple chain transfer agent. Nevertheless the use of RAFT agent leads to low molecular weights close to $10000 \mathrm{~g} \cdot \mathrm{mol}^{-1}$ and much lower polydispersities compared to nitroxide-mediated ethene polymerization (Ip close to 6 for hexyl-DPAIOmediated ethene polymerization ) as shown in Fig. 1.6 where MWDs from RAFT agent and nitroxide-mediated ethene polymerization are compared. According to the fact that only one RAFT agent has been used and that the mechanism of the RAFT mediation is not clearly understood, the use of RAFT agent stays an interesting possibility to be explored with respect to controlling ethene polymerization.

Rizzardo et al. reported, that in the case of a too slow fragmentation of the species $\mathbf{5}$, vinyl acetate polymerization was inhibited. As described before, substituents $\mathrm{R}$ and $\mathrm{Z}$ strongly influence the stability of the intermediate species. Assuming that VAc and ethene have similar reactivities, it is assumed that the intermediate radical 5 will be also formed. The high polymerization rates indicate that $\mathbf{5}$ undergoes a fragmentation reaction, which, however may be different from the type of reaction depicted in Scheme 6.41. 
The first hypothesis is, that the radical $\mathbf{5}$ undergoes thermal decomposition, similar to the alkoxyamine decomposition, presented in nitroxide-mediated ethene polymerization. In order to understand which bond will break, theoretical calculations of the BDE of these C-S and S-hexyl bonds need to be performed.

The second possible explanation for an ineffective RAFT polymerization is that $\mathbf{5}$ decomposes too rapidly. If the radical is too much destabilized by the substituent $\mathrm{Z}$, fragmentation is much more facile and may be too fast. The equilibrium is then strongly shifted to the formation of the propagating radical so that the control is not effective. It may be argued, that substituents which stabilize these radicals would retard their rate of fragmentation. The presence of a phenyl group in the substituent $\mathrm{Z}$ induces a high transfer constant compared to dialkylamine substituent. The electron density on the nitrogen is then lowered by delocalization of the lone pair. Even the substituent $\mathrm{R}$ may be involved in the ineffective RAFT ethene polymerization. Its bulkiness is also an important factor which influences the rate of fragmentation of the intermediate species 2 . In order to slow down the RAFT ethene polymerization, new RAFT agents with different $\mathrm{Z}$ (such as dialkylamine) and $\mathrm{R}$ (less voluminous) substituents should be tested.

A third possibility might be that the intermediate radical $\mathbf{5}$ is capable of adding an ethene molecule and thereby initiating the polymerization.

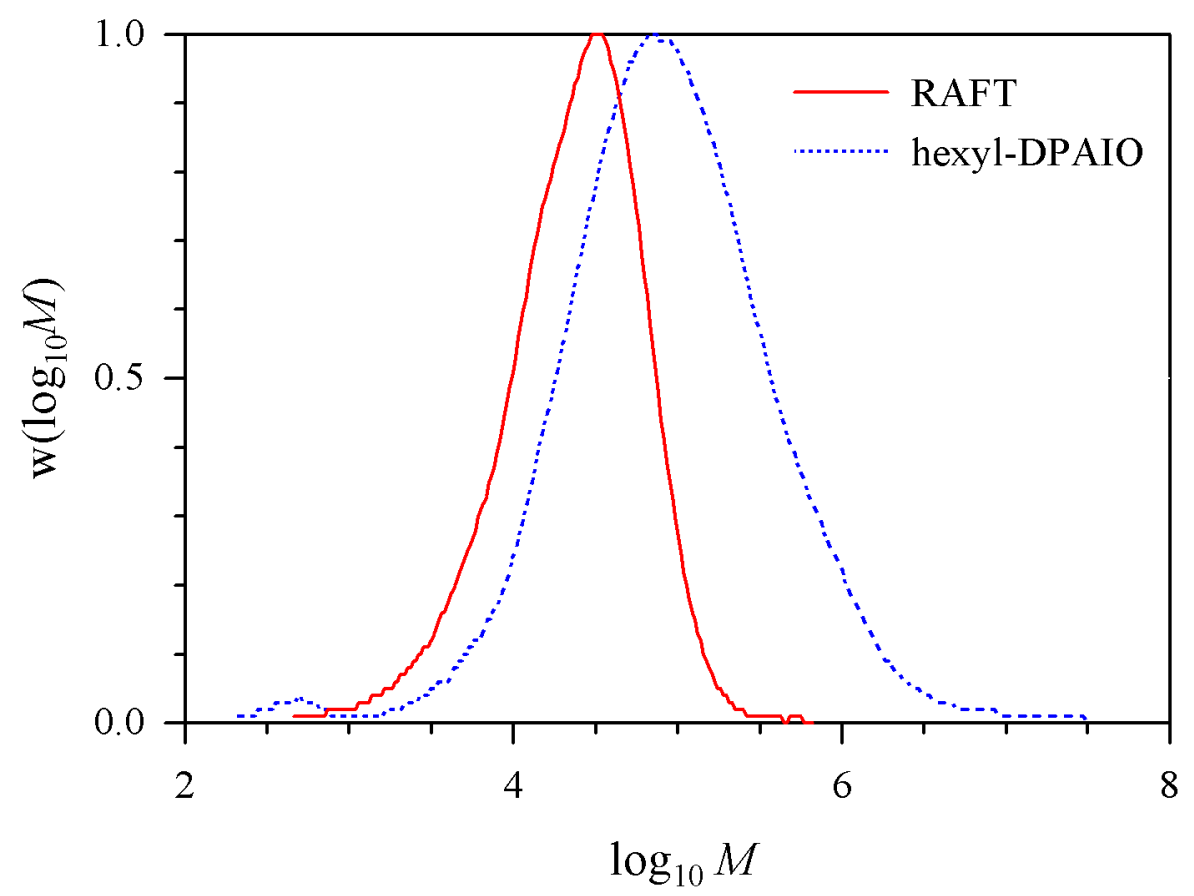

Fig. 6.42. Comparison of MWDs for polyethylene from RAFT ethene polymerization and for a hexyl-DPAIO-mediated ethene polymerization. $P=2000 \mathrm{bar}$, $T=150^{\circ} \mathrm{C},[R A F T] /[E]=200 \mathrm{ppm},[$ hexyl-DPAIO $] /[\mathrm{E}]=200 \mathrm{ppm}$. 


\subsection{References}

[1] M.B. Sderra, Ph. D. Thesis, Göttingen 1997

[2] M. Buback, L. Wittkowski, Z. Phys. Chem. 1999, 210, 61

[3] M. Buback, S. Kligbeil, J. Sandmann, M. B. Sderra, H.P. Vögele, H. Wackerbarth, L. Wittkowski, Z. Phys. Chem. 1999, 210, 199

[4] M. Buback, F. Kuchta, J. Macromol. Chem. Phys. 1995, 196, 1887

[5] M. Buback, F. Kuchta, J. Macromol. Chem. Phys. 1997, 198, 1455

[6] S. Grimaldi, J. Finet, A. Zeghdaoui, P. Tordo, D. Benoit, Y. Gnanou, M. Fontanille, P. Nicol, J.P. Pierson, Am. Chem. Soc. Div. Polym. Chem. 1997, 38, 651

[7] S. Marque, R. Matschke, J. Sobek, H. Fischer, to be published

[8] R. Veregin, M. Georges, K. Daimon, ACS symp. 1998, 685, 170

[9] M. Georges, R. Veregin, P. Kazmaier, G. Hamer, Macromolecules 1994, 27, 7228

[10] R. Veregin, M. Georges, P. Odell, L. Michalk, Macromolecules 1996, 29, 4161

[11] J. He, J. Chen, L. Li, J. Pan, Polymer 2000, 4573

[12] D. Benoit, S. Grimaldi, S. Robin, J.P.Finet, P. Tordo, Y. Gnanou, J. Am. Chem. Soc. 2000, 122,5929

[13] P. Lacroix-Desmazes, J.F. Lutz, B. Boutevin, Macromol. Chem. and Phys. 2000, 201, 662

[14] P. Tordo, Private communications

[15] D. Benoit, S. Grimaldi, S. Robin, J.P.Finet, P. Tordo, Y. Gnanou, J. Am. Chem. Soc. 2000, 122,5929

[16] M. Buback, C. Kowollik, Macromolecules 1998, 31, 3211

[17] S. Bon, G. Chambard, A. L. German, Macromolecules 1999, 32, 8276

[18] D. Benoit, V. Chaplinski, R. Braslau, C. Hawker, J. Am. Chem. Soc. 1999, 16, 3905

[19] K. Matyjaszewski, ACS symph. 1998, 685, 259

[20] J. Wang, K. Matyjaszewski, Macromolecules 1995, 28, 7901

[21] J. Wang, K. Matyjaszewski, J. Am. Chem. Soc. 1995, 117, 5614

[22] T. Patten, J. Xia, T. Abernathy, K. Matyjaszewski, Science 1996, 272, 866

[23] V. Percec, B. Barboiu, Macromolecules 1995, 28, 7970

[24] D. Haddleton, C. Jasieczek, M. Hammon, A Shooter, Macromolecules 1997, 30, 2190

[25] C. Granel, P. Dubois, R. Jerome, P. Teyssie, Macromolecules 1996, 29, 8576

[26] H. Uegaki, Y. Katani, M. Kamigaito, M. Sawamato, Macromolecules 1997, 30, 2249 
[27] M. Kato, M. Kamigaito, M. Sawamato, T. Higashimura, Macromolecules 1995, 28, 1721

[28] J. Wang, K. Matyjaszewski, WO 96/30421 1996

[29] M. Wei, J. Xia, K. Matyjaszewski, Polym. Prepr. (Am. Chem. Soc. Div. Polym. Chem.) 1997, 38(2), 233

[30] M. Wei, J. Xia, N. McDermott, K. Matyjaszewski, Polym. Prepr. (Am. Chem. Soc. Div. Polym. Chem.) 1997, 38(2), 231

[31] P. Lecomte, I. Drapier, P. Dubois, R. Jerome, P. Teyssie, Macromolecules 1997, 24, 7633

[32] M. Wei, J. Xia, N. McDermott, K. Matyjaszewski, Macromolecules 1997, 30, 8161

[33] B. Wayland, L. Basickes, S. Mukerjee, M. Wei, M. Fryd, Macromolecules, 1997, 30, 8109

[34] G. Moineau, C. Granel, P. Dubois, R. Jerome, P. Teyssie, Macromolecules 1998, 31, 542

[35] A. Ajayaghosh, R. Francis, Macromolecules 1998, 31, 1436

[36] K. Matyjaszewski, J. Wang, T. Grimaud, D. Shipp, Macromolecules 1998, 31, 1527

[37] S. Coca, C. Jasieczek, K. Beers, K. Matyjaszewski, J. Polym. Sc. Part A : Polym. Chem. 1998, 36, 1417

[38] H. Schlaad, B. Schmidt, A. Muller, Angewandte Chemie - Int. Ed. 1998, 37, 1389

[39] K. Matyjaszewski, Macromolecules 1998, 31, 4710

[40] J. Xia, S. Gaynor, K. Matyjaszewski, Macromolecules 1998, 31, 5958

[41] G. Cheng, C. Hu, S. Ying, Polymer 1999, 40, 2167

[42] N. Ide, T. Fukuda, Macromolecules 1999, 32, 95

[43] G. Moineau, M. Minet, P. Dubois, R. Jerome, P. Teyssie, T. Senninger, Macromolecules 1999, 32, 27

[44] F. Simal, A. Demonceau, A. Noels, Angewandte Chemie - Int. Ed. 1999, 38, 538

[45] A. Kajiwara, K. Matyjaszewski, Polymer Journal 1999, 31, 70

[46] X. Wang, N. Luo, S. Ying, J. Polym. Sc. Part A : Polym. Chem. 1999, 37, 1255

[47] J. Xia, K. Matyjaszewski, Macromolecules 1999, 32, 2434

[48] F. Simal, A. Demonceau, A. Noels, Tetrahedron Letters 1999, 40, 5689

[49] J. Guo, P. Wu, Z. Han, Chem. J. of Chinese Universities 1999, 30, 1303

[50] D. Shipp, K. Matyjaszewski, Macromolecules 1999, 32, 2948

[51] M. Acar, K. Matyjaszewski, Macromol. Chem. and Ph. 1999, 200, 1094

[52] G. Cheng, C. Hu, S. Ying, J. of Molec. Catalysis - Chemical 1999, 144, 357 
[53] Y. Chong, F. Ercole, G. Moad, E. Rizzardo, S. Thang, Macromolecules 1999, 32, 6895

[54] F. Ercole, G. Moad, C. Johnson, J. Krstina, C. Moad, E. Rizzardo, T. Spurling, S. Thang, A. Anderson, ACS Symp. 1998, 685, 332

[55] P. Tordo, Private communications

[56] S. Beuermann, M. Buback, G. Russel, Macromol. Rapid. Commun. 1994, 15, 351

[57] C. Kowollik, Ph. D. Thesis Göttingen 1999

[58] S. Maeder, R. Gilbert, Macromolecules 1998, 31, 4410

[59] Odian 1970

[60] C. Han, M. Drache, G. Schmidt-Naake, Angew. Makromol. Chem. 1999, 264, 73

[61] P. Tordo, D. Bertin, D. Gigmes, Private communications

[62] L. Greci, M. Depature, Private communications

[63] G. Schmidt-Naake, M. Drache, C.H. Han, A. Koch, Dechema Monographs 1998, 134, 598

[64] A. Alberti, P. Carloni, L. Greci, P. Stipa, C. Neri , Polym. Degrad. And Stab. 1993, 29, 215

[65] W. J. Hehre, L. Radom, P. v. R. Schleyer, J. A. Pople, Ab Initio Molecular Orbital Theory, Wiley: New York, 1986

[66] L. Radom, M. W. Wong, A. Pross, ACS Symp. 1997, 685, 31

[67] P. Tordo, A. Gaudel, Private communications

[68] E. Rizzardo, J. Chiefari, R. T. A. Mayadunne, G. Moad, S. H. Thang, ACS Symp. 2000, 768,278

[69] G. Moad, J. Chiefari, Y. K. Chong, J. Krstina, R. T. A. Mayadunne, , A. Postma, E. Rizzardo, S. H. Thang, Polym. International 2000, in press

[70] K. Moffat, M. Saban, R. Veregin, M. Georges, G. Hamer, P. Kazmaier, U.S. Patent 5 449724,1995 


\section{Closing ReMARKS}

An enormous interest in controlled free-radical polymerization (CRP) has emerged during recent years, with particular emphasis of the polymerization of common monomers such as styrene and acrylates. An adequate understanding of polymerization rate and polymer microstructures for CRP has been reached. Current studies focus on the measurement of combination/dissociation rates in nitroxide-mediated polymerization and on the development of new nitroxides. Also CRP of some unusual monomers has been investigated. With the exception of one patent, no study so far has been devoted to controlled radical polymerization under high pressure. The present study is devoted to CRP under high pressure. Special attention has been paid to high-pressure ethene polymerization, but studies were also carried out for styrene homopolymerization for which an extended body of literature referring to ambient pressure is available.

High-pressure controlled polymerizations of styrene and of butyl acrylate were successfully performed using different nitroxides as mediator. Narrow polydispersities and molecular weights close to the theoretical predictions are observed, irrespective of the applied pressure. High pressure allows for a significant enhancement of polymerization rate. Applying high pressure and using a bulky nitroxide, such as SG1, at $125^{\circ} \mathrm{C}$ induces an approximately nine time faster polymerization rate at 2000 bar as compared to TEMPOmediated styrene polymerization at 1 bar. High pressure also allows to carry out CRP at relatively low temperature and allows to produce high-molecular-weight material with narrow polydispersity. Rate coefficients of the reversible dissociation of the dormant species for several systems were estimated by simulations using the software package PREDICI ${ }^{\circledR}$.

Attempts to control high-pressure ethene polymerization were not successful. Even at fairly high temperature, the dormant species will not readily dissociate. Further increase of temperature is associated with mediator decomposition and with a large enhancement of transfer to monomer reactions. Both effects exclude CRP. Within a joint research program also several new nitroxides have been tested toward application into ethene polymerization, but also without success. PREDICI ${ }^{\circledR}$ simulations and ab-initio calculations allow for the estimation of bond dissociation energies in alkoxyamines. It turns out that in several cases the 
$\mathrm{N}-\mathrm{O}$ bond is less stable than the $\mathrm{C}-\mathrm{O}$ bond which also destroys control of ethene polymerization.

The studies into CRP of ethene were extended to RAFT processes. It was assumed that the $\beta$-scission of the intermediate radical might work more selectively than does bond scission of the alkoxyamines. However the RAFT agent used so far primarily acts as a chaintransfer agent. Nevertheless, it appears that only via a RAFT strategy there may be a chance to control ethene polymerization. 


\section{APPENDIX}

\subsection{Abbreviations}

\begin{tabular}{|c|c|}
\hline AIBN & Azo-bis isobutyronitrile \\
\hline ATRP & Atom Transfer Radical Polymerization \\
\hline $\mathrm{BA}$ & Butyl Acrylate \\
\hline BDE & Bond Dissociation Energy \\
\hline BPO & Dibenzoylperoxide \\
\hline CRP & Controlled free-Radical Polymerization \\
\hline CXA & TEMPO-derived nitroxide (see Section 3.6.1) \\
\hline DPAIO & 3-imino-2-phenyl indolinonic nitroxide (see Section 3.6.1) \\
\hline$D P_{\mathrm{N}}$ & Degree of polymerization \\
\hline DTBN & Di-tert-butylnitroxide (see Section 3.6.1) \\
\hline DTBP & Di-tert-butylperoxide (see Section 3.6.2) \\
\hline$\Delta V^{\neq}$ & Activation volume \\
\hline $\mathrm{E}$ & Ethene \\
\hline$E_{\mathrm{a}}$ & Activation Energy \\
\hline FRP & Free Radical Polymerization \\
\hline hexyl-TEMPO & Alkoxyamine hexyl-TEMPO \\
\hline hexyl-SG1 & Alkoxyamine hexyl-SG1 \\
\hline I & Initiator \\
\hline$I_{p}$ & Polydispersity index \\
\hline$K$ & Overall equilibrium constant $k_{\mathrm{d}} / k_{\mathrm{c}}$ \\
\hline$k_{0}$ & Pre-exponential factor \\
\hline$k_{\mathrm{c}}$ & Rate coefficient of combination of nitroxide and propagating radical \\
\hline$k_{\mathrm{d}}$ & Rate coefficient of alkoxyamine dissociation \\
\hline$k_{\mathrm{dis}}$ & Rate coefficient of disproportionation of nitroxide with propagating radical \\
\hline$k_{\mathrm{p}}$ & Propagation rate coefficient \\
\hline$k_{\mathrm{tc}}$ & Termination rate coefficient for termination by combination \\
\hline$k_{\mathrm{td}}$ & Termination rate coefficient for termination by disproportionation \\
\hline
\end{tabular}


$k_{\mathrm{tr}, \mathrm{M}} \quad$ Transfer to monomer rate coefficient

M Monomer

$v \quad$ wavenumber

MMA Methyl MethAcrylate

$M_{\mathrm{N}} \quad$ Number average molecular weight

$M_{\mathrm{P}} \quad$ Molecular weight at peak maximum

$M_{\mathrm{W}} \quad$ Weight average molecular weight

MWD Molecular Weight Distribution

$\mathrm{N}$

Nitroxide

NMP

Nitroxide-Mediated Polymerization

$P$

Pressure (bar)

PBA PolyButyl Acrylate

PE PolyEthylene

PMMA Poly Methyl MethAcrylate

PR

Persistent Radical

PRE

Persistent Radical Effect

PREDICI $^{\circledR} \quad$ Simulation program PREDICI ${ }^{\circledR}$

PS PolyStyrene

Overall reaction rate

RAFT Radical Addition-Fragmentation Transfer

$\mathrm{R}_{\mathrm{n}}$

Growing macroradical of chain length $n$

$\mathrm{R}_{\mathrm{n}} \mathrm{N}$

Macroradical of chain length $\mathrm{n}$ kept under dormant species

$\mathrm{S}$

Styrene

S-DPAIO Alkoxyamine styryl-DPAIO

SFRP

Stable Free Radical Polymerization

SG1 n-tert-butyl-1-diethylphospono-2,2-dimethylpropyl nitroxide (see Section 3.6.1)

S-SG1 Alkoxyamine styryl-SG1

S-TEMPO Alkoxyamine styryl-TEMPO

$T \quad$ Temperature $(\mathrm{K})$

$\theta \quad$ Temperature $\left({ }^{\circ} \mathrm{C}\right)$

$t \quad$ Time (s)

$t_{90}$

Time at $90 \%$ monomer conversion (s)

TAPP

tert-amylperpivalate (see Section 3.6.2) 
TBPA tert-butylperacetate (see Section 3.6.2)

TEMPO 2,2,6,6-tetramethyl-1-piperidinyloxyl (see Section 3.6.1)

$X$

Monomer conversion

[X] Concentration of species $\mathrm{X}\left(\mathrm{mol} \cdot \mathrm{L}^{-1}\right)$

$[\mathrm{X}]_{0} \quad$ Initial concentration of species $\mathrm{X}\left(\mathrm{mol} \cdot \mathrm{L}^{-1}\right)$ 


\subsection{Experimental molecular weights as a function of monomer conversion for styrene polymerizations}

Molecular weight and polydispersity as a function of monomer conversion for styrene polymerizations in the presence of styryl-TEMPO at $125^{\circ} \mathrm{C}$ and at pressure up to 2000 bar.

\begin{tabular}{ccccccc}
\hline $\begin{array}{c}\text { [styryl-TEMPO] } \\
\text { [styrene] }\end{array}$ & $\begin{array}{c}P \\
\mathrm{bar}\end{array}$ & $\begin{array}{c}\theta \\
{ }^{\circ} \mathrm{C}\end{array}$ & $\begin{array}{c}\text { styrene } \\
\text { conversion/\% }\end{array}$ & $\begin{array}{c}M_{\mathrm{N}} \\
\mathrm{g} \cdot \mathrm{mol}^{-1}\end{array}$ & $\begin{array}{c}M_{\mathrm{W}} \\
\mathrm{g} \cdot \mathrm{mol}^{-1}\end{array}$ & \begin{tabular}{c}
$I p$ \\
\hline $0.101 \%$
\end{tabular} \\
\hline 2000 & 125 & 5.5 & 15400 & 33400 & 2.2 \\
$0.101 \%$ & 2000 & 125 & 9.9 & 20660 & 45800 & 1.95 \\
$0.101 \%$ & 2000 & 125 & 15.6 & 23000 & 38000 & 1.4 \\
$0.101 \%$ & 2000 & 125 & 32.8 & 37700 & 62000 & 1.3 \\
$0.101 \%$ & 2000 & 125 & 40.2 & 44000 & 57500 & 1.3 \\
\hline $0.101 \%$ & 1000 & 125 & 9.9 & 14100 & 29600 & 2.1 \\
$0.101 \%$ & 1000 & 125 & 20.3 & 23300 & 32850 & 1.4 \\
$0.101 \%$ & 1000 & 125 & 31.2 & 34100 & 42600 & 1.25 \\
$0.101 \%$ & 1000 & 125 & 44.8 & 46300 & 57900 & 1.25 \\
\hline $0.101 \%$ & 100 & 125 & 5.7 & 7900 & 21000 & 2.7 \\
$0.101 \%$ & 100 & 125 & 7.8 & 7300 & 15500 & 2.1 \\
$0.101 \%$ & 100 & 125 & 16.8 & 21400 & 27900 & 1.35 \\
$0.101 \%$ & 100 & 125 & 31.7 & 35000 & 41300 & 1.3 \\
$0.101 \%$ & 100 & 125 & 39.8 & 38700 & 48900 & 1.25 \\
\hline
\end{tabular}

Molecular weight and polydispersity as a function of monomer conversion for styrene polymerizations in the presence of styryl-TEMPO at $115^{\circ} \mathrm{C}$ and at $2000 \mathrm{bar}$.

\begin{tabular}{ccccccc}
\hline $\begin{array}{c}\text { [styryl-TEMPO] } \\
\text { [styrene] }\end{array}$ & $\begin{array}{c}P \\
\mathrm{bar}\end{array}$ & $\begin{array}{c}\boldsymbol{} \\
{ }^{\circ} \mathrm{C}\end{array}$ & $\begin{array}{c}\text { styrene } \\
\text { conversion/\% }\end{array}$ & $\begin{array}{c}M_{\mathrm{N}} \\
\mathrm{g} \cdot \mathrm{mol}^{-1}\end{array}$ & $\begin{array}{c}M_{\mathrm{W}} \\
\mathrm{g} \cdot \mathrm{mol}^{-1}\end{array}$ & \multicolumn{1}{c}{} \\
\hline $0.111 \%$ & 2000 & 115 & 9.3 & 14000 & 23800 & 1.7 \\
$0.111 \%$ & 2000 & 115 & 21.6 & 26500 & 39750 & 1.5 \\
$0.111 \%$ & 2000 & 115 & 34.8 & 37400 & 52400 & 1.4 \\
$0.111 \%$ & 2000 & 115 & 46.4 & 43700 & 58100 & 1.3 \\
\hline $0.225 \%$ & 2000 & 115 & 8.7 & 5700 & 11400 & 2.0 \\
$0.225 \%$ & 2000 & 115 & 18.3 & 10300 & 13200 & 1.3 \\
$0.225 \%$ & 2000 & 115 & 30.2 & 16400 & 21650 & 1.3 \\
$0.225 \%$ & 2000 & 115 & 42.1 & 27000 & 35100 & 1.3 \\
\hline
\end{tabular}


Molecular weight and polydispersity as a function of monomer conversion for styrene polymerizations in the presence of styryl-SG1 at $125^{\circ} \mathrm{C}$ and at pressures up to 2000 bar.

\begin{tabular}{ccccccc}
\hline $\begin{array}{c}\text { [styryl-SG1] } \\
\text { [styrene] }\end{array}$ & $\begin{array}{c}P \\
\mathrm{bar}\end{array}$ & $\begin{array}{c}\boldsymbol{}{ }^{\circ} \mathrm{C} \\
\text { conversion/\% }\end{array}$ & $\begin{array}{c}M_{\mathrm{N}} \\
\mathrm{g} \cdot \mathrm{mol}^{-1}\end{array}$ & $\begin{array}{c}M_{\mathrm{W}} \\
\mathrm{g} \cdot \mathrm{mol}^{-1}\end{array}$ & $\begin{array}{c}\text { styrene } \\
\text { conc }\end{array}$ \\
\hline $0.110 \%$ & 2000 & 125 & 6.2 & 31800 & 50300 & 1.55 \\
$0.110 \%$ & 2000 & 125 & 11.0 & 43000 & 56400 & 1.31 \\
$0.110 \%$ & 2000 & 125 & 19.4 & 56950 & 72000 & 1.30 \\
$0.110 \%$ & 2000 & 125 & 28.2 & 69700 & 107350 & 1.27 \\
$0.110 \%$ & 2000 & 125 & 41.3 & 80930 & 126000 & 1.26 \\
\hline $0.110 \%$ & 1000 & 125 & 9.9 & 35600 & 52900 & 1.48 \\
$0.110 \%$ & 1000 & 125 & 19.7 & 53900 & 70000 & 1.30 \\
$0.110 \%$ & 1000 & 125 & 30.4 & 65600 & 82760 & 1.27 \\
\hline $0.110 \%$ & 60 & 125 & 4.8 & 20800 & 31800 & 1.50 \\
$0.110 \%$ & 60 & 125 & 12.0 & 34550 & 48070 & 1.40 \\
$0.110 \%$ & 60 & 125 & 20.0 & 55000 & 74125 & 1.35 \\
$0.110 \%$ & 60 & 125 & 42.5 & 89000 & 117000 & 1.30
\end{tabular}

Molecular weight and polydispersity as a function of monomer conversion for styrene polymerizations in the presence of several concentrations of styryl-SG1 at $120^{\circ} \mathrm{C}$ and at pressures up to 2000 bar.

\begin{tabular}{|c|c|c|c|c|c|c|}
\hline $\begin{array}{c}\text { [styryl-SG1] / } \\
\text { [styrene] }\end{array}$ & $\begin{array}{c}P \\
\text { bar }\end{array}$ & $\begin{array}{c}\theta \\
{ }^{\circ} \mathrm{C}\end{array}$ & $\begin{array}{c}\text { styrene } \\
\text { conversion/\% }\end{array}$ & $\begin{array}{c}M_{\mathrm{N}} \\
\mathrm{g} \cdot \mathrm{mol}^{-1}\end{array}$ & $\begin{array}{c}M_{\mathrm{W}} \\
\mathrm{g} \cdot \mathrm{mol}^{-1}\end{array}$ & $I p$ \\
\hline $0.282 \%$ & 2000 & 120 & 19.8 & 11900 & 1500 & 1.26 \\
\hline $0.282 \%$ & 2000 & 120 & 42.5 & 22250 & 26700 & 1.20 \\
\hline $0.282 \%$ & 2000 & 120 & 57.6 & 30100 & 36400 & 1.21 \\
\hline $0.282 \%$ & 2000 & 120 & 73.6 & 39300 & 49900 & 1.27 \\
\hline $0.282 \%$ & 50 & 120 & 20.1 & 12200 & 1590 & 1.30 \\
\hline $0.282 \%$ & 50 & 120 & 40.0 & 21500 & 27950 & 1.30 \\
\hline $0.282 \%$ & 50 & 120 & 58.3 & 33100 & 45350 & 1.37 \\
\hline $0.282 \%$ & 50 & 120 & 70.0 & 35400 & 48500 & 1.37 \\
\hline $0.114 \%$ & 2000 & 120 & 5.5 & 31000 & 52700 & 1.70 \\
\hline $0.114 \%$ & 2000 & 120 & 10.6 & 40800 & 65700 & 1.61 \\
\hline
\end{tabular}




\begin{tabular}{ccccccc}
$0.114 \%$ & 2000 & 120 & 11.2 & 32000 & 50250 & 1.57 \\
$0.114 \%$ & 2000 & 120 & 21.8 & 64800 & 84250 & 1.30 \\
$0.114 \%$ & 2000 & 120 & 31.7 & 84000 & 105850 & 1.26 \\
$0.114 \%$ & 2000 & 120 & 32.0 & 86000 & 111800 & 1.30 \\
$0.114 \%$ & 2000 & 120 & 44.0 & 94000 & 125000 & 1.33 \\
$0.114 \%$ & 2000 & 120 & 53.6 & 110000 & 148500 & 1.35 \\
\hline $0.114 \%$ & 50 & 120 & 9.0 & 33700 & 43500 & 1.29 \\
$0.114 \%$ & 50 & 120 & 18.9 & 44000 & 66450 & 1.51 \\
$0.114 \%$ & 50 & 120 & 25.5 & 55300 & 82950 & 1.50 \\
$0.114 \%$ & 50 & 120 & 30.7 & 63000 & 91350 & 1.45 \\
$0.114 \%$ & 50 & 120 & 41.6 & 84000 & 136900 & 1.63 \\
$0.114 \%$ & 50 & 120 & 50.5 & 79000 & 129550 & 1.64 \\
$0.114 \%$ & 50 & 120 & 60.2 & 94000 & 145700 & 1.55 \\
\hline $0.074 \%$ & 2000 & 120 & 9.5 & 42600 & 61750 & 1.45 \\
$0.074 \%$ & 2000 & 120 & 24.0 & 92400 & 129350 & 1.40 \\
$0.074 \%$ & 2000 & 120 & 37.6 & 115000 & 161000 & 1.40 \\
$0.074 \%$ & 2000 & 120 & 53.1 & 137000 & 209600 & 1.53 \\
$0.074 \%$ & 2000 & 120 & 57.5 & 147000 & 219000 & 1.49 \\
\hline $0.074 \%$ & 50 & 120 & 7.2 & 40300 & 69300 & 1.72 \\
$0.074 \%$ & 50 & 120 & 14.8 & 66500 & 96400 & 1.45 \\
$0.074 \%$ & 50 & 120 & 23.8 & 76800 & 119000 & 1.55 \\
$0.074 \%$ & 50 & 120 & 24.9 & 77200 & 120400 & 1.56 \\
$0.074 \%$ & 50 & 120 & 32.5 & 87000 & 140950 & 1.62 \\
$0.074 \%$ & 50 & 120 & 50.5 & 84000 & 130200 & 1.55 \\
$0.074 \%$ & 50 & 120 & 55.0 & 91500 & 161000 & 1.76 \\
\hline & & & & & &
\end{tabular}


Molecular weight and polydispersity as a function of monomer conversion for styrene polymerizations in the presence of several concentrations of styryl-SG1 at $100^{\circ} \mathrm{C}$ and at 2000 bar.

\begin{tabular}{|c|c|c|c|c|c|c|}
\hline $\begin{array}{c}\text { [styryl-SG1] / } \\
\text { [styrene] }\end{array}$ & $\begin{array}{c}P \\
\text { bar }\end{array}$ & $\begin{array}{c}\theta \\
{ }^{\circ} \mathrm{C}\end{array}$ & $\begin{array}{c}\text { styrene } \\
\text { conversion/\% }\end{array}$ & $\begin{array}{c}M_{\mathrm{N}} \\
\mathrm{g} \cdot \mathrm{mol}^{-1}\end{array}$ & $\begin{array}{c}M_{\mathrm{W}} \\
\mathrm{g} \cdot \mathrm{mol}^{-1}\end{array}$ & Ip \\
\hline $0.220 \%$ & 2000 & 100 & 20.0 & 12500 & 17000 & 1.36 \\
\hline $0.220 \%$ & 2000 & 100 & 38.2 & 26000 & 33800 & 1.30 \\
\hline $0.220 \%$ & 2000 & 100 & 55.2 & 34000 & 43200 & 1.27 \\
\hline $0.220 \%$ & 2000 & 100 & 9.8 & 6500 & 9250 & 1.42 \\
\hline $0.114 \%$ & 2000 & 100 & 9.8 & 25700 & 41900 & 1.63 \\
\hline $0.114 \%$ & 2000 & 100 & 20.3 & 37250 & 52150 & 1.40 \\
\hline $0.114 \%$ & 2000 & 100 & 34.5 & 52100 & 67750 & 1.30 \\
\hline $0.114 \%$ & 2000 & 100 & 50.2 & 70300 & 88600 & 1.26 \\
\hline
\end{tabular}

Molecular weight and polydispersity as a function of monomer conversion for styrene polymerizations in the presence of styryl-DPAIO at pressures up to 2000 bar.

\begin{tabular}{|c|c|c|c|c|c|c|}
\hline $\begin{array}{c}\text { [styryl-DPAIO] / } \\
\text { [styrene] }\end{array}$ & $\begin{array}{c}P \\
\text { bar }\end{array}$ & $\begin{array}{c}\theta \\
{ }^{\circ} \mathrm{C}\end{array}$ & $\begin{array}{c}\text { styrene } \\
\text { conversion/\% }\end{array}$ & $\begin{array}{c}M_{\mathrm{N}} \\
\mathrm{g} \cdot \mathrm{mol}^{-1}\end{array}$ & $\begin{array}{c}M_{\mathrm{W}} \\
\mathrm{g} \cdot \mathrm{mol}^{-1}\end{array}$ & $I p$ \\
\hline $0.100 \%$ & 2000 & 125 & 5 & 130000 & 246000 & 1.90 \\
\hline $0.100 \%$ & 2000 & 125 & 15 & 139000 & 260000 & 1.87 \\
\hline $0.100 \%$ & 2000 & 125 & 30 & 132000 & 255000 & 1.94 \\
\hline $0.100 \%$ & 2000 & 125 & 47 & 127000 & 169000 & 1.93 \\
\hline $0.100 \%$ & 100 & 125 & 5 & 39300 & 118500 & 3.01 \\
\hline $0.100 \%$ & 100 & 125 & 16 & 60900 & 110000 & 1.80 \\
\hline $0.100 \%$ & 100 & 125 & 31 & 42000 & 81200 & 1.85 \\
\hline $0.100 \%$ & 100 & 125 & 46 & 35000 & 81200 & 2.32 \\
\hline $0.100 \%$ & 2000 & 140 & 19.7 & 110000 & 190000 & 1.73 \\
\hline $0.100 \%$ & 2000 & 140 & 43.7 & 105000 & 184000 & 1.70 \\
\hline $0.100 \%$ & 2000 & 140 & 55 & 90700 & 160000 & 1.75 \\
\hline $0.100 \%$ & 50 & 140 & 20.3 & 66000 & 112000 & 1.70 \\
\hline $0.100 \%$ & 50 & 140 & 39.5 & 62700 & 108600 & 1.73 \\
\hline $0.100 \%$ & 50 & 140 & 53.6 & 64500 & 110000 & 1.75 \\
\hline
\end{tabular}


Molecular weight and polydispersity as a function of monomer conversion for styrene polymerizations in the presence of styryl-DPAIO and $0.01 \%$ DPAIO at $140^{\circ} \mathrm{C}$ and at pressures up to 2000 bar.

\begin{tabular}{ccccccc}
\hline $\begin{array}{c}\text { [styryl-DPAIO] / } \\
\text { [styrene] }\end{array}$ & $\begin{array}{c}P \\
\text { bar }\end{array}$ & $\begin{array}{c}\theta \\
{ }^{\circ} \mathrm{C}\end{array}$ & $\begin{array}{c}\text { styrene } \\
\text { conversion/\% }\end{array}$ & $\begin{array}{c}M_{\mathrm{N}} \\
\mathrm{g} \cdot \mathrm{mol}^{-1}\end{array}$ & $\begin{array}{c}M_{\mathrm{W}} \\
\mathrm{g} \cdot \mathrm{mol}^{-1}\end{array}$ & $I p$ \\
\hline $0.100 \%+0.01 \%$ & 2000 & 140 & 11.3 & 25570 & 43460 & 1.70 \\
$0.100 \%+0.01 \%$ & 2000 & 140 & 22.8 & 33260 & 80940 & 2.43 \\
\hline $0.100 \%+0.01 \%$ & 1000 & 140 & 9.2 & 28762 & 89150 & 3.10 \\
$0.100 \%+0.01 \%$ & 1000 & 140 & 20.3 & 28170 & 91770 & 3.25 \\
\hline $0.100 \%+0.01 \%$ & 100 & 140 & 9.9 & 22400 & 59650 & 2.65 \\
$0.100 \%+0.01 \%$ & 100 & 140 & 15.7 & 46450 & 112900 & 2.42 \\
\hline
\end{tabular}

Molecular weight and polydispersity as a function of monomer conversion for styrene polymerizations in the presence of DPAIO at $140^{\circ} \mathrm{C}$ and at pressures up to 2000 bar. $[D P A I O] /[A I B N]=2.5$.

\begin{tabular}{|c|c|c|c|c|c|c|}
\hline $\begin{array}{l}\text { [AIBN] / } \\
\text { [styrene] }\end{array}$ & $\begin{array}{c}P \\
\text { bar }\end{array}$ & $\begin{array}{c}\theta \\
{ }^{\circ} \mathrm{C}\end{array}$ & $\begin{array}{c}\text { styrene } \\
\text { conversion/\% }\end{array}$ & $\begin{array}{c}M_{\mathrm{N}} \\
\mathrm{g} \cdot \mathrm{mol}^{-1}\end{array}$ & $\begin{array}{c}M_{\mathrm{W}} \\
\mathrm{g} \cdot \mathrm{mol}^{-1}\end{array}$ & $I p$ \\
\hline $0.050 \%$ & 2000 & 140 & 15.7 & 45800 & 101300 & 2.21 \\
\hline $0.050 \%$ & 2000 & 140 & 37.6 & 71100 & 146000 & 2.05 \\
\hline $0.050 \%$ & 100 & 140 & 16.8 & 46100 & 90700 & 1.97 \\
\hline $0.050 \%$ & 100 & 140 & 28.7 & 63300 & 127000 & 2.00 \\
\hline $0.050 \%$ & 100 & 140 & 40.0 & 59700 & 127600 & 2.14 \\
\hline
\end{tabular}




\subsection{Experimental molecular weights as a function of monomer conversion for (meth)acrylate polymerizations}

Molecular weight and polydispersity as a function of monomer conversion for butyl acrylate polymerizations in the presence of styryl-SG1 at $125^{\circ} \mathrm{C}$ and at pressures up to 2000 bar.

\begin{tabular}{ccccccc}
\hline $\begin{array}{c}\text { [styryl-SG1]/ } \\
{[B A]}\end{array}$ & $\begin{array}{c}P \\
\text { bar }\end{array}$ & $\begin{array}{c}\theta \\
{ }^{\circ} \mathrm{C}\end{array}$ & $\begin{array}{c}\mathrm{BA} \\
\text { conversion/\% }\end{array}$ & $\begin{array}{c}M_{\mathrm{N}} \\
\mathrm{g} \cdot \mathrm{mol}^{-1}\end{array}$ & $\begin{array}{c}M_{\mathrm{W}} \\
\mathrm{g} \cdot \mathrm{mol}^{-1}\end{array}$ & $\begin{array}{c}I p \\
0.110 \%\end{array}$ \\
\hline 2000 & 125 & 10.3 & 29000 & & 1.65 \\
$0.110 \%$ & 2000 & 125 & 20.0 & 44500 & 71200 & 1.60 \\
$0.110 \%$ & 2000 & 125 & 28.3 & 61000 & 100040 & 1.64 \\
$0.110 \%$ & 2000 & 125 & 38.9 & 65000 & 100100 & 1.44 \\
$0.110 \%$ & 2000 & 125 & 51.0 & 97000 & 133100 & 1.37 \\
\hline $0.110 \%$ & 60 & 125 & 20.0 & 42300 & 67680 & 1.60 \\
$0.110 \%$ & 60 & 125 & 31.0 & 60500 & 92565 & 1.53 \\
$0.110 \%$ & 60 & 125 & 42.2 & 72000 & 108000 & 1.50 \\
$0.110 \%$ & 60 & 125 & 58.8 & 83200 & 124800 & 1.50 \\
\hline
\end{tabular}

Molecular weight and polydispersity as a function of monomer conversion for methyl methacrylate polymerizations in the presence of styryl-SG1 at $95^{\circ} \mathrm{C}$ and at pressures up to 2000 bar.

\begin{tabular}{|c|c|c|c|c|c|c|}
\hline $\begin{array}{c}\text { [styryl-SG1] / } \\
{[M M A]}\end{array}$ & $\begin{array}{c}P \\
\text { bar }\end{array}$ & $\begin{array}{c}\theta \\
{ }^{\circ} \mathrm{C}\end{array}$ & $\begin{array}{c}\text { MMA } \\
\text { conversion/\% }\end{array}$ & $\begin{array}{c}M_{\mathrm{N}} \\
\mathrm{g} \cdot \mathrm{mol}^{-1}\end{array}$ & $\begin{array}{c}M_{\mathrm{W}} \\
\mathrm{g} \cdot \mathrm{mol}^{-1}\end{array}$ & $I p$ \\
\hline $0.101 \%$ & 50 & 95 & 22.2 & 129000 & 271900 & 2.1 \\
\hline $0.101 \%$ & 50 & 95 & 28.5 & 115000 & 204700 & 1.8 \\
\hline $0.101 \%$ & 50 & 95 & 34.9 & 119000 & 221300 & 1.9 \\
\hline $0.101 \%$ & 2000 & 95 & 35.0 & 213000 & 445170 & 2.1 \\
\hline $0.101 \%$ & 2000 & 95 & 35.1 & 211000 & 438880 & 2.1 \\
\hline $0.101 \%$ & 2000 & 95 & 55.8 & 248000 & 508400 & 2.0 \\
\hline
\end{tabular}


Molecular weight and polydispersity as a function of monomer conversion for methyl methacrylate polymerizations in the presence of $\mathrm{CN}-\mathrm{DPAIO}$ at $95^{\circ} \mathrm{C}$ and at $2000 \mathrm{bar}$.

\begin{tabular}{ccccccc}
\hline $\begin{array}{c}{[C N-D P A I O] /} \\
{[M M A]}\end{array}$ & $\begin{array}{c}P \\
\text { bar }\end{array}$ & $\begin{array}{c}\theta \\
{ }^{\circ} \mathrm{C}\end{array}$ & $\begin{array}{c}\text { MMA } \\
\text { conversion/\% }\end{array}$ & $\begin{array}{c}M_{\mathrm{N}} \\
\mathrm{g} \cdot \mathrm{mol}^{-1}\end{array}$ & $\begin{array}{c}M_{\mathrm{W}} \\
\mathrm{g} \cdot \mathrm{mol}^{-1}\end{array}$ & $\begin{array}{c}I p \\
0.103 \%\end{array}$ \\
\hline 2000 & 95 & 13.0 & 164000 & 474000 & 2.9 \\
$0.103 \%$ & 2000 & 95 & 24.5 & 147000 & 324000 & 2.2 \\
$0.103 \%$ & 2000 & 95 & 38.7 & 120000 & 305000 & 2.6 \\
\hline
\end{tabular}

Molecular weight and polydispersity as a function of monomer conversion for conventional methyl methacrylate polymerizations initiated by AIBN at $95^{\circ} \mathrm{C}$ and at $2000 \mathrm{bar}$.

\begin{tabular}{ccccccc}
\hline$[A I B N] /[M M A]$ & $\begin{array}{c}P \\
\text { bar }\end{array}$ & $\begin{array}{c}\theta \\
{ }^{\circ} \mathrm{C}\end{array}$ & $\begin{array}{c}\text { MMA } \\
\text { conversion/\% }\end{array}$ & $\begin{array}{c}M_{\mathrm{N}} \\
\mathrm{g} \cdot \mathrm{mol}^{-1}\end{array}$ & $\begin{array}{c}M_{\mathrm{W}} \\
\mathrm{g} \cdot \mathrm{mol}^{-1}\end{array}$ & $\begin{array}{c}I p \\
\end{array}$ \\
\hline $0.052 \%$ & 50 & 95 & 29.3 & 77000 & 141000 & 1.8 \\
\hline $0.052 \%$ & 2000 & 95 & 50.5 & 130000 & 306000 & 2.6 \\
\hline
\end{tabular}




\subsection{Experimental molecular weights as a function of monomer conversion for ethene polymerizations}

Molecular weight and polydispersity as a function of monomer conversion for ethene polymerizations in the presence of styryl-SG1 at $190^{\circ} \mathrm{C}$ and at $2000 \mathrm{bar}$.

\begin{tabular}{ccccccc}
\hline $\begin{array}{c}\text { [styryl-SG1] } \\
\text { / ethene] }\end{array}$ & $\begin{array}{c}P \\
\mathrm{bar}\end{array}$ & $\begin{array}{c}\boldsymbol{C} \\
{ }^{\circ} \mathrm{C}\end{array}$ & $\begin{array}{c}\text { ethene } \\
\text { conversion/\% }\end{array}$ & $\begin{array}{c}M_{\mathrm{N}} \\
\mathrm{g} \cdot \mathrm{mol}^{-1}\end{array}$ & $\begin{array}{c}M_{\mathrm{W}} \\
\mathrm{g} \cdot \mathrm{mol}^{-1}\end{array}$ & \multicolumn{1}{l}{} \\
\hline $53 \mathrm{ppm}$ & 2000 & 190 & 10.6 & 37950 & 111900 & 2.9 \\
$53 \mathrm{ppm}$ & 2000 & 190 & 16.4 & 32280 & 104300 & 3.3 \\
$53 \mathrm{ppm}$ & 2000 & 190 & 19.4 & 34300 & 127100 & 3.7 \\
$53 \mathrm{ppm}$ & 2000 & 190 & 19.6 & 37320 & 112300 & 3.0 \\
\hline $107 \mathrm{ppm}$ & 2000 & 190 & 12.2 & 27420 & 64050 & 2.3 \\
$107 \mathrm{ppm}$ & 2000 & 190 & 12.5 & 26590 & 64600 & 2.4 \\
$107 \mathrm{ppm}$ & 2000 & 190 & 25.0 & 25910 & 64090 & 2.5 \\
$107 \mathrm{ppm}$ & 2000 & 190 & 31.0 & 26960 & 90430 & 3.3 \\
$107 \mathrm{ppm}$ & 2000 & 190 & 20.0 & 25250 & 82480 & 3.2 \\
\hline $200 \mathrm{ppm}$ & 2000 & 190 & 6.3 & 12740 & 63230 & 4.9 \\
$200 \mathrm{ppm}$ & 2000 & 190 & 10.3 & 13060 & 53810 & 4.1 \\
$200 \mathrm{ppm}$ & 2000 & 190 & 11.5 & 16890 & 53960 & 3.2 \\
$200 \mathrm{ppm}$ & 2000 & 190 & 16.7 & 17320 & 81150 & 4.7 \\
\hline
\end{tabular}

Molecular weight and polydispersity as a function of monomer conversion for ethene polymerizations in the presence of TEMPO at $200^{\circ} \mathrm{C}$ and at 2000 bar. [TEMPO] / [DTBP] = 2.

\begin{tabular}{ccccccc}
\hline $\begin{array}{c}\text { [DTBP] } \\
\text { [ethene] }\end{array}$ & $\begin{array}{c}P \\
\text { bar }\end{array}$ & $\begin{array}{c}\theta \\
{ }^{\circ} \mathrm{C}\end{array}$ & $\begin{array}{c}\text { ethene } \\
\text { conversion/\% }\end{array}$ & $\begin{array}{c}M_{\mathrm{N}} \\
\mathrm{g} \cdot \mathrm{mol}^{-1}\end{array}$ & $\begin{array}{c}M_{\mathrm{W}} \\
\mathrm{g} \cdot \mathrm{mol}^{-1}\end{array}$ & $\begin{array}{c}I p \\
50 \mathrm{ppm}\end{array}$ \\
\hline 2000 & 200 & 12.8 & 21590 & 99314 & 4.6 \\
$50 \mathrm{ppm}$ & 2000 & 200 & 15.6 & 24250 & 94575 & 3.9 \\
$50 \mathrm{ppm}$ & 2000 & 200 & 18.0 & 14790 & 72471 & 4.9 \\
$50 \mathrm{ppm}$ & 2000 & 200 & 20.9 & 11770 & 62381 & 5.3 \\
$50 \mathrm{ppm}$ & 2000 & 200 & 21.6 & 19920 & 99600 & 5.0 \\
$50 \mathrm{ppm}$ & 2000 & 200 & 38.2 & 13620 & 126666 & 9.3 \\
$50 \mathrm{ppm}$ & 2000 & 200 & 48.0 & 14000 & 112000 & 8.0 \\
$50 \mathrm{ppm}$ & 2000 & 200 & 50.0 & 16100 & 127000 & 7.9 \\
\hline
\end{tabular}


Molecular weight and polydispersity as a function of monomer conversion for ethene polymerizations in the presence of CXA at $220^{\circ} \mathrm{C}$ and at 2000 bar. $[C X A] /[D T B P]=1.1$.

\begin{tabular}{ccccccc}
\hline $\begin{array}{c}\text { [DTBP] } \\
\text { [ethene] }\end{array}$ & $\begin{array}{c}P \\
\text { bar }\end{array}$ & $\begin{array}{c}\theta \\
{ }^{\circ} \mathrm{C}\end{array}$ & $\begin{array}{c}\text { ethene } \\
\text { conversion/\% }\end{array}$ & $\begin{array}{c}M_{\mathrm{N}} \\
\mathrm{g} \cdot \mathrm{mol}^{-1}\end{array}$ & $\begin{array}{c}M_{\mathrm{W}} \\
\mathrm{g} \cdot \mathrm{mol}^{-1}\end{array}$ & $\begin{array}{c}I p \\
50 \mathrm{ppm}\end{array}$ \\
\hline 2000 & 220 & 3.3 & 28000 & 148400 & 5.3 \\
$50 \mathrm{ppm}$ & 2000 & 220 & 8.7 & 23200 & 132240 & 5.7 \\
$50 \mathrm{ppm}$ & 2000 & 220 & 14.3 & 24800 & 178560 & 7.2 \\
\hline
\end{tabular}

Molecular weight and polydispersity as a function of monomer conversion for ethene polymerizations in the presence of hexyl-TEMPO at 2000 bar and at temperatures between 210 and $250^{\circ} \mathrm{C}$.

\begin{tabular}{ccccccc}
\hline $\begin{array}{c}\text { [hexyl-TEMPO]/ } \\
\text { [ethene] }\end{array}$ & $\begin{array}{c}P \\
\mathrm{bar}\end{array}$ & $\begin{array}{c}\theta \\
{ }^{\circ} \mathrm{C}\end{array}$ & $\begin{array}{c}\text { ethene } \\
\text { conversion/\% }\end{array}$ & $\begin{array}{c}M_{\mathrm{N}} \\
\mathrm{g} \cdot \mathrm{mol}^{-1}\end{array}$ & $\begin{array}{c}M_{\mathrm{W}} \\
\mathrm{g} \cdot \mathrm{mol}^{-1}\end{array}$ & Ip \\
\hline $100 \mathrm{ppm}$ & 2000 & 230 & 34.7 & 22920 & 108700 & 4.7 \\
$100 \mathrm{ppm}$ & 2000 & 230 & 42.8 & 12910 & 117200 & 9.1 \\
$100 \mathrm{ppm}$ & 2000 & 230 & 43.4 & 12170 & 83660 & 6.9 \\
$100 \mathrm{ppm}$ & 2000 & 230 & 48.8 & 13490 & 86710 & 6.4 \\
$100 \mathrm{ppm}$ & 2000 & 230 & 55.0 & 24270 & 274000 & 11.3 \\
$100 \mathrm{ppm}$ & 2000 & 230 & 64.0 & 13270 & 134000 & 10.1 \\
\hline $100 \mathrm{ppm}$ & 2000 & 210 & 4.5 & 11400 & 69480 & 6.1 \\
$100 \mathrm{ppm}$ & 2000 & 210 & 9.2 & 14900 & 80500 & 5.4 \\
$100 \mathrm{ppm}$ & 2000 & 210 & 12.3 & 8318 & 93940 & 11.3 \\
$100 \mathrm{ppm}$ & 2000 & 210 & 14.0 & 16480 & 103700 & 6.2 \\
\hline $100 \mathrm{ppm}$ & 2000 & 250 & 28.1 & 9657 & 71950 & 7.5 \\
$100 \mathrm{ppm}$ & 2000 & 250 & 39.8 & 6912 & 73870 & 10.7 \\
$100 \mathrm{ppm}$ & 2000 & 250 & 48.9 & 7380 & 106900 & 14.5 \\
$100 \mathrm{ppm}$ & 2000 & 250 & 55.4 & 5994 & 74830 & 12.5 \\
\hline
\end{tabular}


Molecular weight and polydispersity as a function of monomer conversion for ethene polymerizations in the presence of hexyl-DPAIO at 2000 bar and at temperatures between 140 and $170^{\circ} \mathrm{C}$.

\begin{tabular}{|c|c|c|c|c|c|c|}
\hline $\begin{array}{c}\text { [hexyl-DPAIO] / } \\
\text { [ethene] }\end{array}$ & $\begin{array}{c}P \\
\text { bar }\end{array}$ & $\begin{array}{c}\theta \\
{ }^{\circ} \mathrm{C}\end{array}$ & $\begin{array}{c}\text { ethene } \\
\text { conversion/\% }\end{array}$ & $\begin{array}{c}M_{\mathrm{N}} \\
\mathrm{g} \cdot \mathrm{mol}^{-1}\end{array}$ & $\begin{array}{c}M_{\mathrm{W}} \\
\mathrm{g} \cdot \mathrm{mol}^{-1}\end{array}$ & Ip \\
\hline $100 \mathrm{ppm}$ & 2000 & 140 & 14.5 & 62080 & 234400 & 3.8 \\
\hline $100 \mathrm{ppm}$ & 2000 & 150 & 11.9 & 40240 & 216200 & 5.4 \\
\hline $100 \mathrm{ppm}$ & 2000 & 160 & 7.2 & 45470 & 167000 & 3.7 \\
\hline 100 ppm & 2000 & 160 & 11.9 & 40240 & 216200 & 5.4 \\
\hline 100 ppm & 2000 & 160 & 14.3 & 48330 & 246500 & 5.1 \\
\hline $100 \mathrm{ppm}$ & 2000 & 160 & 14.8 & 47870 & 264800 & 5.5 \\
\hline $100 \mathrm{ppm}$ & 2000 & 160 & 18.3 & 37820 & 236200 & 6.3 \\
\hline 100 ppm & 2000 & 160 & 37.8 & 55100 & 407500 & 7.4 \\
\hline $100 \mathrm{ppm}$ & 2000 & 170 & 5.5 & 28000 & 75000 & 2.7 \\
\hline 100 ppm & 2000 & 170 & 18.8 & 31990 & 175300 & 5.5 \\
\hline 100 ppm & 2000 & 170 & 23.0 & 40000 & 244600 & 6.1 \\
\hline $12 \mathrm{ppm}$ & 2000 & 160 & 5.2 & 99730 & 338000 & 3.4 \\
\hline $12 \mathrm{ppm}$ & 2000 & 160 & 8.6 & 106700 & 348700 & 3.3 \\
\hline $25 \mathrm{ppm}$ & 2000 & 160 & 5.3 & 69760 & 190800 & 2.7 \\
\hline $25 \mathrm{ppm}$ & 2000 & 160 & 10.0 & 70460 & 234100 & 3.3 \\
\hline $25 \mathrm{ppm}$ & 2000 & 160 & 16.2 & 68210 & 206300 & 3.0 \\
\hline $50 \mathrm{ppm}$ & 2000 & 160 & 7.5 & 48190 & 188600 & 3.9 \\
\hline $50 \mathrm{ppm}$ & 2000 & 160 & 13.8 & 52640 & 256200 & 4.9 \\
\hline 50 ppm & 2000 & 160 & 18.7 & 53750 & 296500 & 5.5 \\
\hline $50 \mathrm{ppm}$ & 2000 & 160 & 25.8 & 54270 & 326300 & 6.0 \\
\hline $100 \mathrm{ppm}+10 \%$ & 2000 & 160 & 5.6 & 36360 & 87600 & 2.4 \\
\hline $100 \mathrm{ppm}+10 \%$ & 2000 & 160 & 10.3 & 34360 & 106700 & 3.1 \\
\hline $100 \mathrm{ppm}+10 \%$ & 2000 & 160 & 17.7 & 37370 & 129200 & 3.5 \\
\hline $100 \mathrm{ppm}+10 \%$ & 2000 & 160 & 18.3 & 33900 & 119100 & 3.5 \\
\hline $150 \mathrm{ppm}$ & 2000 & 160 & 8.8 & 30950 & 104000 & 3.4 \\
\hline 150 ppm & 2000 & 160 & 11.4 & 27800 & 109800 & 3.9 \\
\hline 150 ppm & 2000 & 160 & 19.6 & 31690 & 139400 & 4.4 \\
\hline 150 ppm & 2000 & 160 & 21.5 & 32100 & 147300 & 4.6 \\
\hline $200 \mathrm{ppm}$ & 2000 & 160 & 7.4 & 15920 & 105700 & 6.6 \\
\hline
\end{tabular}




\begin{tabular}{lllllll}
$200 \mathrm{ppm}$ & 2000 & 160 & 13.0 & 16920 & 143400 & 8.5 \\
$200 \mathrm{ppm}$ & 2000 & 160 & 19.5 & 19360 & 151400 & 7.8 \\
$200 \mathrm{ppm}$ & 2000 & 160 & 23.6 & 19250 & 174200 & 9.0 \\
\hline $400 \mathrm{ppm}$ & 2000 & 160 & 10.6 & 21670 & 85720 & 4.4 \\
$400 \mathrm{ppm}$ & 2000 & 160 & 14.9 & 25860 & 100000 & 3.9 \\
$400 \mathrm{ppm}$ & 2000 & 160 & 21.5 & 26000 & 123300 & 4.7 \\
\hline
\end{tabular}

Molecular weight and polydispersity as a function of monomer conversion for RAFT ethene polymerizations at 2000 bar and at temperatures between 150 and $170^{\circ} \mathrm{C}$.

\begin{tabular}{|c|c|c|c|c|c|c|}
\hline $\begin{array}{l}\text { [RAFT] / } \\
\text { [ethene] }\end{array}$ & $\begin{array}{c}P \\
\text { bar }\end{array}$ & $\begin{array}{c}\theta \\
{ }^{\circ} \mathrm{C}\end{array}$ & $\begin{array}{c}\text { ethene } \\
\text { conversion/\% }\end{array}$ & $\begin{array}{c}M_{\mathrm{N}} \\
\mathrm{g} \cdot \mathrm{mol}^{-1}\end{array}$ & $\begin{array}{c}M_{\mathrm{W}} \\
\mathrm{g} \cdot \mathrm{mol}^{-1}\end{array}$ & $I p$ \\
\hline $204 \mathrm{ppm}$ & 2000 & 150 & 8.5 & 10040 & 20830 & 2.1 \\
\hline 204 ppm & 2000 & 150 & 18.8 & 12310 & 32330 & 2.7 \\
\hline 204 ppm & 2000 & 150 & 20.3 & 13250 & 27880 & 2.2 \\
\hline 204 ppm & 2000 & 150 & 25.4 & 12910 & 39330 & 2.4 \\
\hline 204 ppm & 2000 & 150 & 31.7 & 15200 & 37010 & 2.6 \\
\hline 198 ppm & 2000 & 160 & 14.0 & 11070 & 27120 & 2.4 \\
\hline 198 ppm & 2000 & 160 & 39.1 & 12870 & 35800 & 3.0 \\
\hline $208 \mathrm{ppm}$ & 2000 & 170 & 10.0 & 7040 & 25730 & 3.7 \\
\hline $104 \mathrm{ppm}$ & 2000 & 150 & 18.8 & 24670 & 54030 & 2.2 \\
\hline
\end{tabular}




\section{ACKNOWLEDGMENTS}

To my supervisors, Prof. Dr. Buback (University of Göttingen) and Dr. Senninger (ATOFINA), I am indebted for the foray into scientific research which I have enjoyed, and learnt from. Thank you for encouragement and interest throughout.

My especial thanks are due to Dr. DesCourières (ATOFINA), Prof. Dr. Greci (University of Ancona) and Prof. Dr. Tordo (University of Marseille) for their helpful discussions within a joint research project.

At the practical level, many thanks to Dr. Hans Peter Vögele for his company and also for his ability to keep everything running. Véronique El Rezzi and Dr. Vögele are thanked for their vigilant proof reading and advice on the preparation of this thesis. My thanks to the "Buback Abteilung" as a whole for the pleasant and cooperative working environment, especially to Heike Rohmann for her friendship and her sense of humor.

It is a pleasure to acknowledge the cooperation which has been enjoyed with ATOFINA (Groupement de Recherche de Lacq, France) in the course of this work. The SEC analysis of polyethylene samples is gratefully acknowledged. I am also indebted to ATOFINA for financial support throughout my Ph.D. tenue.

I am grateful to Prof. Dr. K. Matyjaszewski and Prof. Dr. H. Fischer for valuable discussions.

Enfin je tiens a remercier tout particulièrement Véronique pour les bons moments passés à Göttingen et son soutien pendant la durée de ce travail. 


\section{Curriculum Vitae}

\section{Personal information}

02.07.1974

Born in Remiremont, France

\section{Education \& qualification}
$1980-1985 \quad$ Elementary School in Epinal, France
1985 - 1992 GCE A Levels in Maths, Physics/Chemistry Biology, History Louis Lapicque High School, Epinal
$1992-1994$ Technological University Institute of Chemistry, University of Lille, France
$1994-1997 \quad$ Engineer's diploma (Chemistry) in Lille Superior Chemistry School "ENSCL"
Option : materials science and metallurgy

Since Dec. 1997 Ph. D. in collaboration with ATOFINA at the Institute for Physical Chemistry, in the research group of prof. M. Buback, University of Göttingen. Theme : study and simulation of free-radical polymerization (ethylene, styrene, acrylates) in the presence of persistent radicals under high pressure

\section{Previous experience}

May-June 1994 Technical training period with AKZO-NOBEL, Montataire, France : study of the influence of different compounds on paint rheology

Summer 1995 Technical training period with UNION MINIERE, Auby, France : study of zinc precipitation in basic conditions

Summer 1996 Technical training period with E.R.C., Fresnes, France : soil analysis development following AFNOR standards

April - Sept. 1997 End-of-term project with CERDATO (ATOFINA), Serquigny, France : study of the relation structures-properties of polyurethane dispersions and production scale-up in a 1501 reactor

Oct. - Nov. 1997 Scientific research work at ATOFINA, Lacq, France : study of initiator decomposition and polymerization under high pressure

Since Dec. 1997 Research toward doctorate supported by ATOFINA at the University of Göttingen 